Sobre a Existência de Infinitas

Geodésicas Fechadas em

Good Orbifolds Riemannianos

Pablo Asdrúbal Díaz Sepúlveda

TESE APRESENTADA

$\mathrm{AO}$

Instituto DE MATEMÁticA E EstatísticA

DA

UNIVERSIDADE DE SÃo PAUlo

PARA

OBTENÇÃO DO TÍTULO

$\mathrm{DE}$

DOUTOR EM CIÊNCIAS

Programa: Matemática

Orientador: Prof. Dr. Marcos Martins Alexandrino da Silva

Durante o desenvolvimento deste trabalho o autor recebeu auxílio financeiro da CAPES/CNPq

São Paulo, março de 2018 
Sobre a Existência de Infinitas

Geodésicas Fechadas em

Good Orbifolds Riemannianos

Esta é a versão original da dissertação/tese elaborada pelo candidato Pablo Asdrúbal Díaz Sepúlveda, tal como submetida à Comissão Julgadora. 


\title{
Sobre a Existência de Infinitas \\ Geodésicas Fechadas em \\ Good Orbifolds Riemannianos
}

\begin{abstract}
Esta versão da dissertação/tese contém as correções e alterações sugeridas pela Comissão Julgadora durante a defesa da versão original do trabalho, realizada em 06/04/2018. Uma cópia da versão original está disponível no Instituto de Matemática e Estatística da Universidade de São Paulo.
\end{abstract}

Comissão Julgadora:

- Prof. Dr. Marcos Martin Alexandrino da Silva (orientador) - IME-USP

- Prof. Dr. Miguel Angel Javaloyes Victoria - UM - Externo

- Prof. Dr. Benigno Oliveira Alves - Externo

- Prof. Dr. Francisco Jose Gozzi - IME - Externo

- Prof. Dr. Icaro Gonçalves - IME - Externo 


\title{
AGRADECIMENTOS
}

\author{
«Thank you for the inspiration \\ Thank you for the smiles \\ All the unconditional love \\ That carried me for miles \\ It carried me for miles \\ But most of all thank you for \\ my life.» \\ -Mike Portnoy (Dream Theater)
}

Para todos aqueles que foram, direta e indiretamente, parte do processo durante este trabalho. Muito obrigado! 
Para Teddy: meu fiel amigo que sempre esperou meu regresso. 


\section{RESUMO}

DÍAZ SEPÚLVEDA PABLO ASDRÚBAL, Sobre a Existência de Infinitas Geodésicas Fechadas em Good Orbifolds Riemannianos. 2018. Tese (Doutorado) - Instituto de Matemática e Estatística, Universidade de São Paulo, São Paulo, 2018.

Nesta tese demonstramos, entre outras coisas, a existência de infinitas geodésicas fechadas em good orbifolds Riemannianos $M / \Gamma$, onde $\Gamma$ é um grupo de isometrias virtualmente Abeliano. No caso particular onde $\Gamma$ é um produto semi-direto de um grupo finito por um grupo Abeliano, concluimos a existência de uma família de geodésicas fechadas com comprimentos tendendo a infinito.

Palavras-chave: Good Orbifold, Geodésica Fechada, Geometricamente Distintas. 


\section{ABSTRACT}

DÍAZ SEPÚLVEDA PABLO ASDRÚBAL, On the Existence of Infinitely Many Closed Geodesics in Good Riemannian Orbifolds. 2018. Tese (Doutorado) - Instituto de Matemática e Estatística, Universidade de São Paulo, São Paulo, 2018.

In this $\mathrm{PhD}$ theses we prove, among other things, the existence of infinity many (geometric distinct) closed geodesics on good Riemannian compact orbifolds $M / \Gamma$, where $\Gamma$ is a virtual abelian group of isometries. In the particular case where $\Gamma$ is a semi-direct product of a finite group with an abelian group, we also assure that there isa family of closed geodesics for which the lengths tend to infinity.

Keywords: Good Orbifold, Closed Geodesic, Geometrically Distinct. 


\section{SUMÁRIO}

$\begin{array}{ll}\text { Introdução } & 1\end{array}$

1 Preliminares $\quad 3$

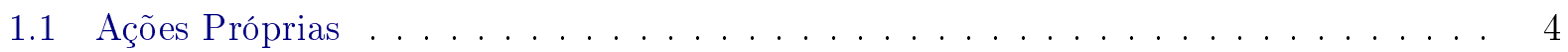

1.2 Ações Descontínuas . . . . . . . . . . . . . . . . . . . . 7

1.3 Domínios Fundamentais . . . . . . . . . . . . . . . . . . . . . . 15

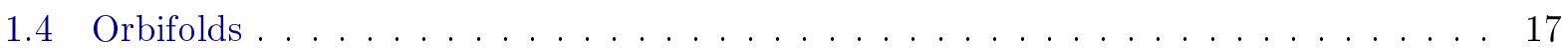

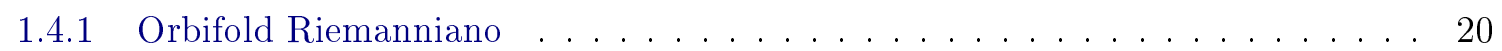

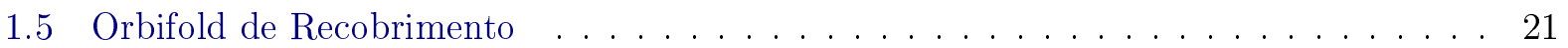

1.6 O Pseudogrupo de Mudança de Cartas . . . . . . . . . . . . . . . . . . . 24

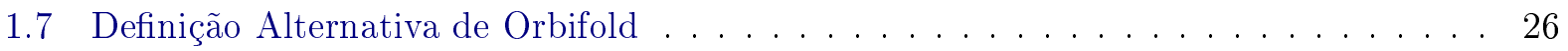

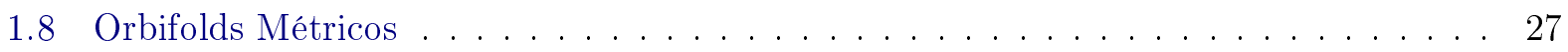

2 Existência de Geodésicas fechadas num Orbifold Riemanniano 45

2.1 Geodésica Fechada num Orbifold Riemanniano . . . . . . . . . . . . . . . . . . . . . 46

2.2 Ações Cocompactas e Função Deslocamento . . . . . . . . . . . . . . . . . . . . . 48

2.3 Processo de Duplo Encurtamento . . . . . . . . . . . . . . . . . . . . . 52

2.4 Existência de Geodésicas Fechadas num Orbifold de Curvatura Não-Negativa ou

Não-Positiva . . . . . . . . . . . . . . . . . . . . . . 59

3 Resultados $\quad \mathbf{6 5}$

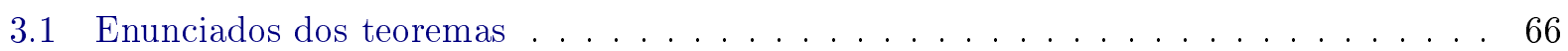

3.2 Ideias das Provas dos Teoremas e das Proposições . . . . . . . . . . . . . . . . . . 69

3.2 .1 Ideia da Prova do Teorema 1 (3.4.1). . . . . . . . . . . . . . . . . 69

3.2 .2 Ideia da Prova do Teorema $2(3.5 .1) \ldots \ldots \ldots \ldots \ldots \ldots \ldots \ldots$

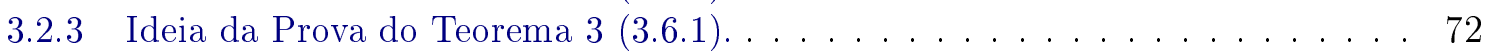

3.2 .4 Prova do Corolário $1 \ldots \ldots \ldots \ldots \ldots \ldots$

3.3 Métrica da Palavra e Função Deslocamento . . . . . . . . . . . . . . . . . . . 76

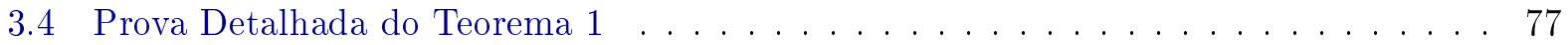

3.5 Prova Detalhada do Teorema $2 \ldots \ldots \ldots \ldots \ldots$

3.6 Prova Detalhada do Teorema $3 \ldots \ldots \ldots 1$

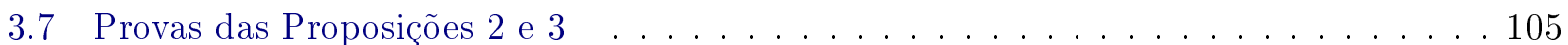

3.8 O caso do Triângulo Equilátero: uma prova alternativa . . . . . . . . . . . . . . 119

$\begin{array}{lr}\text { Referências Bibliográficas } & 129\end{array}$ 


\section{INTRODUÇÃO}

Nesta tese estudamos a existência de infinitas geodésicas geométricamente distintas em good orbifolds.

Para nós um Good orbifold Riemanniano deve ser visto como o quociente $X=M / \Gamma$ de uma variedade Riemanniana simplesmente conexa $M$ (the developed space) por um grupo fechado de isometrias $\Gamma$ (vide definições no Capítulo 1)

Diremos que uma geodésica não constante $\alpha:[0,1] \rightarrow M$ no nosso developed space $M$ é chamada geodésica fechada do good orbifold $X$ se existe $\omega \in \Gamma$, tal que

$$
\omega \alpha(0)=\alpha(1) \text { e } d \omega \dot{\alpha}(0)=\dot{\alpha}(1) .
$$

Assim dizemos que duas geodésicas fechadas $\left(\omega_{1},\left.\alpha_{1}\right|_{[0,1]}\right)$ e $\left(\omega_{2},\left.\alpha_{2}\right|_{[0,1]}\right)$ são e iguais em $X$ se e somente se

$$
\operatorname{imagem}\left(\pi\left(\alpha_{1}\right)\right)=\operatorname{imagem}\left(\pi\left(\alpha_{2}\right)\right) \text {. }
$$

Onde $\pi: M \longrightarrow M / \Gamma$ é a aplicação quociente.

Os resultados principais desta tese sobre geodésicas fechadas encontram-se formulados e demonstrados no capítulo 3 .

Um dos nossos principais resultados é formulado no Teorema 2, teorema onde vamos garantir que: dado um good orbifold Riemanniano compacto $X=M / \Gamma$ (dimensão de $M$ é maior do que 1) onde $\Gamma$ é virtualmente abeliano, então $X$ admite infinitas geodésicas fechadas geometricamente distintas. 
Para tanto precisamos do Teorema 1, o qual a grosso modo descreve uma relação entre geodésicas fechadas de $X$ com outro orbifold Riemanniano "maior".

No caso particular onde $\Gamma$ é um grupo abeliano $\Gamma_{0}$ podemos concluir no Teorema 3 que existe uma sequencia de infinitas geodésicas fechadas em $X=M / \Gamma_{0}$ com comprimentos que tendem ao infinito.

Como consequência, concluímos no Corolário 1 que se $\Gamma$ é um produto semi-direto de um grupo finito $\tilde{\Gamma}$ por um grupo Abeliano $\Gamma_{0}$, então existe uma sequência de infinitas geodésicas fechadas geometricamente distintas em $X=M / \Gamma$ com comprimentos que tendem ao infinito.

De fato como observado na Seção 3.3 (Proposição 3) é possível descrever de forma mais precisa o crescimento de tal sequencia de geodésicas fechadas, e.g., relacionando seus comprimentos com o a métrica da palavra. Tal descrição será possível por meio da Proposição 2, a qual, no caso em que o grupo $\Gamma$ é um grupo Abeliano $\Gamma_{0}$, apresenta uma formula que relaciona a métrica da palavra com a função deslocamento.

Sendo assim, esta tese está organizada da seguinte forma:

No capítulo 1 apresentamos os pré-requisitos necessários, fixando notações e resultados clássicos. Também discutimos no capítulo 1 definições alternativas de orbifolds Riemannianos em particular a definição dada por Lytchak de orbifolds métricos.

No capítulo 2 discutimos alguns resultados na literatura sobre existência de geodésicas fechadas em orbifolds Riemannianos. Em particular recordamos resultados de M. Alexandrino e M.A. Javaloyes sobre shorting process e existência de geodésicas fechadas em espaços folheados bem como geodésicas em orbifolds sobre hipóteses de curvatura (vide [Dra14]).

Finalmente no capítulo 3 apresentamos os resultados principais da tese, discutidos rapidamente acima. Além disto fornecemos na seção 3.8 uma prova alternativa dos resultados principais do capítulo 3 para o caso particular onde $M$ é o espaço Euclidiano $\mathbb{R}^{2}$ (com uma métrica não necessariamente euclidiana) e $W$ é um grupo de Coxeter Euclidiano. Como veremos na Proposição 3.8.1, neste caso particular poderemos inferir novas propriedades da família das infinitas geodésicas. 


\section{CAPÍTULO 1}

\section{PRELIMINARES}

«Jamais considere seus estudos como uma obrigação, mas como uma oportunidade invejável para aprender a conhecer a influência libertadora da beleza do reino do espírito, para seu próprio prazer pessoal e para proveito da comunidade à qual seu futuro trabalho pertence.»

-Albert Einstein

Neste capítulo, vamos apresentar uma introdução de ações próprias, ações descontínuas, domínios fundamentais e orbifolds (seções 1.1, 1.2, 1.3 e 1.4, resp.); en cada seção daremos algumas definições e resultados clássicos que serão úteis neste Capítulo e nos Capítulos 2 e 3. As referências da Seção 1.1 são [AB15] e [DK00], da Seção 1.2 são [Rat06] e [Lee11], da Seção 1.3 é [Rat06] e da Seção 1.4 são [Dra11] e [P.02].

Na Seção 1.7 daremos uma definição alternativa de orbifold a partir do conceito de pseudogrupo de mudança de cartas, conceito que será definido na Seção 1.6. As referências nesta Seção são [AB15] e [AJ11]. Por último, na Seção 1.8 daremos uma prova das Proposições 1.8.2 e 1.8.3, resultados que são citados de umas notas do professor Claudio Gorodski (A metric approach to representations of compact Lie groups). 


\subsection{Ações Próprias}

Uma ação de um grupo $\Gamma$ sobre um espaço $M$ é um homomorfismo $\mathfrak{A}$ de $\Gamma$ no grupo das transformações de $M$. Quando $\Gamma$ é um grupo de Lie e $M$ é uma variedade diferencíavel, então a ação é de classe $C^{k}$ se a aplicação $\mathfrak{A}: \Gamma \times M \rightarrow M$ é de classe $C^{k}$. No nosso caso, sempre vamos supor que a ação é de classe $C^{\infty}$, e dizemos que a ação é suave.

Definição 1.1.1 Seja $\Gamma$ um grupo de Lie e $M$ uma variedade diferenciável. Uma aplicação suave $\mu: \Gamma \times M \rightarrow M$ é chamada uma ação à esquerda de $\Gamma$ sobre $M$ ou uma $\Gamma$-ação à esquerda sobre $M$, se

1. $\mu(e, x)=x$, para todo $x \in M$, onde e é o elemento identidade de $\Gamma$;

2. $\mu\left(\omega_{1},\left(\omega_{2}, x\right)\right)=\mu\left(\omega_{1} \omega_{2}, x\right)$, para cada par $\omega_{1}, \omega_{2} \in \Gamma$ e para todo $x \in M$.

Geralmente denotaremos $\mu(\omega, x)$ por $\omega(x)$. Similarmente podemos definir uma ação à direita $\mu: M \times \Gamma \rightarrow M$ satisfazendo as mesmas condições acima descritas.

Alguns exemplos de ações podem ser vistos em [AB15] (Capítulo 3).

Definição 1.1.2 Sejam $\mu: \Gamma \times M \rightarrow M$ uma ação à esquerda e $x \in M$. Definimos o subgrupo

$$
\Gamma_{x}:=\{\omega \in \Gamma: \mu(\omega, x)=x\} \subset \Gamma
$$

e é chamado o grupo de isotropia de $x$, e o conjunto

$$
\Gamma(x):=\{\omega(x): \omega \in \Gamma\} \subset M
$$

é chamado a órbita de $x$.

O subgrupo $\bigcap_{x \in M} \Gamma_{x}$ é chamado o kernel ineficaz da ação. Se $\bigcap_{x \in M} \Gamma_{x}=\{e\}$, então dizemos que a ação é efetiva. Mais ainda, se $\Gamma_{x}=\{e\}$, para cada $x \in M$, então dizemos que a ação é livre. Se para cada par $x, y \in M$, existe um $\omega \in \Gamma$ tal que $\mu(\omega, x)=y$, então a ação é chamada transitiva.

Dada uma ação suave à esquerda $\mu: \Gamma \times M \rightarrow M$, definimos as seguintes aplicações auxiliares

$$
\begin{aligned}
& \mu^{\omega}: M \longrightarrow M \quad \mu_{x}: \Gamma \longrightarrow M \\
& x \longmapsto \mu(\omega, x) \quad \omega \longmapsto \mu(\omega, x)
\end{aligned}
$$


A ideia principal de uma ação à esquerda (respetivamente à direita) é que cada $\omega \in \Gamma$ determina uma transformacão de $M$, a saber $\mu^{\omega}: M \rightarrow M$. Dado que vamos supor que as ações são de classe $C^{\infty}$, temos que $\mu^{\omega}$ é um elemento de $\operatorname{Dif}(M)$, e assim $\mu^{\Gamma}:=\left\{\mu^{g}: g \in G\right\}$ é um subgrupo de Dif $(M)$.

Definição 1.1.3 Sejam $\mu: \Gamma \times M \rightarrow M$ uma ação e g uma métrica Riemanniana em $M$. Dizemos que a ação é por isometrias ou, simplesmente, isométrica, se $\mu^{\Gamma}:=\left\{\mu^{\omega}: \omega \in \Gamma\right\}$ é um subgrupo de Iso $(M, g)$. Neste caso dizemos que a métrica é $\Gamma$-invariante.

Definição 1.1.4 Seja $\mu: \Gamma \times M \rightarrow M$ uma ação. Dizemos que a ação é própria, ou que $\Gamma$ age propriamente sobre $M$, se a seguinte aplicação

$$
\begin{aligned}
\tilde{\mu}: \Gamma \times M & \longrightarrow M \times M \\
(\omega, x) & \longmapsto(x, \mu(\omega, x))
\end{aligned}
$$

é própria, i.e., a pré-imagem pela aplicação $\tilde{\mu}$ de cada compacto em $M \times M$ é compacto em $\Gamma \times M$.

Observação 1.1.1 Da definicão 1.1.4 temos de forma imediata que se $\mu: \Gamma \times M \rightarrow M$ é uma ação própria, então para cada $x \in M$ o grupo $\Gamma_{x}$ é compacto. Assim, em especial, se $\Gamma$ é discreto, temos que $\Gamma_{x}$ é finito, para cada $x \in M$.

Proposição 1.1.1 Sejam $\mu: \Gamma \times M \rightarrow M$ uma ação própria e $M$ uma variedade compacta, então $\Gamma$ é um grupo de Lie compacto.

Demonstração. Dado que $M$ é uma variedade compacta e $\mu: \Gamma \times M \rightarrow M$ é uma ação própria, temos que $\tilde{\mu}^{-1}(M \times M)=\Gamma \times M$ é compacto. Usando novamente que $M$ é compacta obtemos que $\Gamma$ é compacto.

Proposição 1.1.2 Uma ação $\mu: \Gamma \times M \rightarrow M$ é própria se e somente se dadas duas sequências $\left\{\omega_{i}\right\}_{i \in \mathbb{N}}$ e $\left\{x_{i}\right\}_{i \in \mathbb{N}}$ em $\Gamma$ e $M$, respectivamente, tais que $\left\{x_{i}\right\}_{i \in \mathbb{N}}$ e $\left\{\mu\left(\omega_{i}, x_{i}\right)\right\}_{i \in \mathbb{N}}$ são convergentes em $M$, então a sequência $\left\{\omega_{i}\right\}_{i \in \mathbb{N}}$ admite uma subsequência convergente.

Demonstração. Primeiro vamos supor que a ação $\mu: \Gamma \times M \rightarrow M$ é própria. Sejam $\left\{\omega_{i}\right\}_{i \in \mathbb{N}}$ e $\left\{x_{i}\right\}_{i \in \mathbb{N}}$ duas sequências em $\Gamma$ e $M$, respectivamente, e tais que $\left\{x_{i}\right\}_{i \in \mathbb{N}}$ e $\left\{\mu\left(\omega_{i}, x_{i}\right)\right\}_{i \in \mathbb{N}}$ convergem em $M$. Vamos supor que $\lim _{n \rightarrow \infty} x_{n}=x_{0}$ e $\lim _{n \rightarrow \infty} \mu\left(\omega_{n}, x_{n}\right)=y_{0}$, então os conjuntos $\left\{x_{0}, x_{1}, x_{2}, \ldots\right\}$ e $\left\{y_{0}, \mu\left(\omega_{1}, x_{1}\right), \mu\left(\omega_{2}, x_{2}\right), \ldots\right\}$ são compactos em $M$, e portanto, definindo $K:=\left\{x_{0}, x_{1} \ldots\right\} \times$ $\left\{y_{0}, \mu\left(\omega_{1}, x_{1}\right), \mu\left(\omega_{2}, x_{2}\right), \ldots\right\}$, temos que $K$ é compacto em $M \times M$. Assim, dado que a ação é 
própria, temos que $\tilde{\mu}^{-1}(K)$ é compacto em $\Gamma \times M$. Agora,

$$
\tilde{\mu}^{-1}(K)=\{(\omega, x) \in \Gamma \times M:(x, \mu(\omega, x)) \in K\} .
$$

Portanto $\left\{\left(\omega_{i}, x_{i}\right)\right\}_{i \in \mathbb{N}}$ está contida em $\tilde{\mu}^{-1}(K)$, que é compacto, logo $\left\{\left(\omega_{i}, x_{i}\right)\right\}_{i \in \mathbb{N}}$ possui uma subsequência convergente, e assim $\left\{\omega_{i}\right\}_{i \in \mathbb{N}}$ admite uma subsequência convergente.

Vamos provar a recíproca. Dado $A$ compacto em $M \times M$, queremos provar que $\tilde{\mu}^{-1}(A)$ é compacto em $\Gamma \times M$. Agora, $\tilde{\mu}^{-1}(A)=\{(\omega, x):(x, \mu(\omega, x)) \in A\}$, assim, dado que $A$ é compacto temos, que se $\left.\left\{\left(\omega_{i}, x_{i}\right)\right)\right\}_{i \in \mathbb{N}}$ é uma sequência em $\tilde{\mu}^{-1}(A)$, então a sequência $\left\{\left(x_{i}, \mu\left(\omega_{i}, x_{i}\right)\right)\right\}_{i \in \mathbb{N}}$ admite uma subsequência convergente. Em particular, as sequências $\left\{x_{i}\right\}_{i \in \mathbb{N}}$ e $\left\{\mu\left(\omega_{i}, x_{i}\right)\right\}_{i \in \mathbb{N}}$ admitem subsequências convergentes. Logo, usando a hipôtese, temos que, passando pelas subsequências convergentes de $\left\{x_{i}\right\}_{i \in \mathbb{N}}$ e $\left\{\mu\left(\omega_{i}, x_{i}\right)\right\}_{i \in \mathbb{N}},\left\{\omega_{i}\right\}_{i \in \mathbb{N}}$ tem uma susequência convergente. Portanto $\tilde{\mu}^{-1}(A)$ é sequencialmente compacto.

Da proposição anterior obtemos o seguinte resultado

Corolário 1.1.1 Sejam $\Gamma$ um grupo de Lie compacto e $\mu: \Gamma \times M \rightarrow M$ uma ação, então a ação é própria.

Proposição 1.1.3 Sejam Y um espaço topológico Hausdorff e localmente compacto e $\Gamma$ um subgrupo discreto de Homeo $(Y)$. Se $\Gamma$ age propriamente sobre $Y$, então temos que

1. O espaço $Y / \Gamma$ é um Hausdorff com a topologia quociente;

2. para cada $x \in Y$, temos que $\Gamma_{x}$ é finito;

3. para cada $x \in Y$, existe um aberto $U_{x} \subset Y$ contendo $x$ tal que

$$
\left\{\omega \in \Gamma: \omega\left(U_{x}\right) \cap U_{x} \neq \varnothing\right\}=\Gamma_{x} .
$$

Demonstração. Seja $\pi: M \rightarrow M / \Gamma$ a aplicação quociente. É fácil ver que $\pi$ é aberta. Para provar que $M / \Gamma$ é Hausdorff basta provar que o conjunto

$$
D:=\{(x, y) \in Y \times Y: y \in \Gamma(x)\}
$$

é fechado em $Y \times Y$. Seja $\left\{\left(x_{i}, y_{i}\right)\right\}_{i \in \mathbb{N}}$ é uma sequência em $D$ tal que $\lim _{i \rightarrow \infty} x_{i}=x_{0}$ e $\lim _{i \rightarrow \infty} y_{i}=y_{0}$. Agora, para cada $i \in \mathbb{N}$, existe $\omega_{i} \in \Gamma$ tal que $\omega_{i}\left(x_{i}\right)=y_{i}$, pois $\left\{\left(x_{i}, y_{i}\right)\right\}_{i \in \mathbb{N}}$ uma sequência em $D$. 
Dado que $\Gamma$ age propriamente e usando a Proposição 1.1.2, temos que a sequência $\left\{\omega_{i}\right\}_{i \in \mathbb{N}}$ admite uma subsequência convergente $\left\{\omega_{i_{j}}\right\}_{j \in \mathbb{N}}$, e vamos supor que converge a $\omega_{0} \in \Gamma$. Dado que $\Gamma$ é discreto, temos que, salvo finitos termos, $\omega_{i_{j}}=\omega_{0}$. Assim

$$
\omega_{0}\left(x_{0}\right)=\lim _{j \rightarrow \infty} \omega_{0}\left(x_{i_{j}}\right)=\lim _{j \rightarrow \infty} \omega_{i_{j}}\left(x_{i_{j}}\right)=\lim _{j \rightarrow \infty} y_{i_{j}}=y_{0} .
$$

Portanto $\omega_{0}\left(x_{0}\right)=y_{0}$, e assim $\left(x_{0}, y_{0}\right)$ é um elemento de $D$. Então $D$ é fechado em $Y \times Y$, e temos o item número 1.

Dado que $\Gamma$ age propriamente sobre $Y$ e pela Observação 1.1.1, temos que cada $\Gamma_{x}$ é finito. Assim temos o item 2 .

Agora, sejam $K_{1}=\{x\}$ e $K_{2}$ uma vizinhança compacta de $x$. Dado que $\Gamma$ age propriamente sobre $Y$, temos que o conjunto $\left\{\omega \in \Gamma: \omega(x) \in K_{2}\right\}$ é finito, e é facil ver que contém o subgrupo $\Gamma_{x}$. Assim, podemos encontrar outra vizinhança compacta de $x$, digamos $K_{3} \subset K_{2}$ tal que $\{\omega \epsilon$ $\left.\Gamma: \omega(x) \in K_{3}\right\}=\Gamma_{x}$. Definamos agora $K:=\bigcup_{\omega \in \Gamma_{x}} \omega\left(K_{3}\right)$, então $K$ é uma vizinhança compacta de $x$ e é $\Gamma_{x}$-invariante. Usando novamente que a ação de $\Gamma$ é própria, obtemos que o conjunto $\{\omega \in \Gamma: \omega(K) \cap K \neq \varnothing\}$ é finito e contém o subgrupo $\Gamma_{x}$. Podemos encontrar uma vizinhança $x \in V_{x} \subset K$ tal que $\left\{\omega \in \Gamma: \omega\left(V_{x}\right) \cap V_{x} \neq \varnothing\right\}=\Gamma_{x}$. Agora, definamos $U_{x}:=\bigcup_{\omega \in \Gamma_{x}} \omega\left(V_{x}\right)$, e assim $U_{x}$ é a vizinhaça desejada. E assim temos 3 .

\subsection{Ações Descontínuas}

Nesta seção, diferente da seção anterior, o espaço onde vamos aplicar a ação de um grupo, é um espaço métrico e não uma variedade.

Definição 1.2.1 Um grupo $\Gamma$ age descontinuamente sobre um espaço métrico $(Y, d)$ se para cada compacto $K$ em $Y$ temos que $\{\omega \in \Gamma: \omega K \cap K \neq \varnothing\}$ é um subconjunto finito de $\Gamma$. Se $\Gamma$ age por homeomorfimos ou por isometrias sobre $(Y, d)$, então dizemos que $\Gamma$ age descontinuamente sobre $Y$ se a ação é descontínua.

Lema 1.2.1 Se $\Gamma$ age descontinuamente sobre um espaço métrico $(Y, d)$, então o grupo de isotropia de cada ponto em $Y$ é um subgrupo finito de $\Gamma$.

Demonstração. Seja $x \in Y$, então $\{x\}$ é compacto. Assim, dado que $\Gamma$ age descontinuamente sobre $Y$, temos que $\Gamma_{x}$ é finito. 
Lema 1.2.2 Sejam $(Y, d)$ um espaço métrico e $\Gamma$ um grupo que age descontinuamente por isometrias sobre $Y$. Então cada $\Gamma$-órbita é um subconjunto fechado e discreto de $Y$.

Demonstração. Primeiro observemos que se $\Gamma$ é finito então obtemos o resultado imediatamente. Assim, vamos supor que $\Gamma$ é infinito. Seja $x \in Y$, e vamos provar que $\Gamma(x)$ é localmente finito, e vamos provar isto pelo absurdo. Supomos que $\Gamma(x)$ não é localmente finito, i.e., para $y \in Y$ e para toda vizinhança $U$ de $y$ temos que $U$ contém infinitos pontos de $\Gamma(x)$. Então existe uma sequência infinita $\left\{\omega_{i}\right\}_{i \in \mathbb{N}}$ de elementos distintos de $\Gamma$ tais que a sequência $\left\{\omega_{i}(x)\right\}_{i \in \mathbb{N}}$ converge para $y$. Assim, o conjunto

$$
K:=\left\{x, y, \omega_{1}(x), \omega_{2}(x), \ldots\right\}
$$

é um subconjunto compacto de $Y$. Dado que $\omega_{i}(x) \in K \cap \omega_{i} K$, para todo $i \in \mathbb{N}$, então temos um absurdo, pois $\Gamma$ age descontínuamente sobre $Y$. Portanto $\{\{\omega(x)\}: \omega \in \Gamma\}$ é uma família de fechados de $Y$ locamente finita. Assim, cada subconjunto de $\Gamma(x)$ é fechado em $Y$, em particular $\Gamma(x)$ é discreto e fechado.

Teorema 1.2.1 Seja $\Gamma$ um grupo que age por isometrias sobre um espaço métrico $(Y, d)$. Então $\Gamma$ age descontinuamente sobre $Y$ se e somente se

1. cada grupo de isotropia é finito e

2. cada $\Gamma$-órbita é um subconjunto discreto e fechado de $Y$.

Demonstração. Suponhamos que $\Gamma$ age descontinuamente sobre $Y$. Então, pelos Lemas 1.2.1 e 1.2.2, temos os itens 1 e 2 , respectivamente.

Vamos provar a recíproca do teorema. Primeiro observe que se $\Gamma$ é finito, então a recíproca é imediata. Então vamos supor que cada grupo de isotropia é finito e que cada $\Gamma$ órbita é um subconjunto fechado e discreto de $Y$, mas vamos supor que $\Gamma$ não age descontinuamente sobre $Y$, e desejamos obter um absurdo. Assim, existe um subconjunto compacto $K$ em $Y$ e uma sequência $\left\{\omega_{i}\right\}_{i \in \mathbb{N}}$ de elementos diferentes de $\Gamma$ tal que $\omega_{i}(K) \cap K \neq \varnothing$, para cada $i \in \mathbb{N}$. Logo, para cada $i \in \mathbb{N}$ existe $x_{i} \in K$ tal que $\omega_{i}\left(x_{i}\right) \in K$. Agora, dado que $K$ é compacto, existe uma subsequência $\left\{x_{i_{j}}\right\}_{j \in \mathbb{N}}$ de $\left\{x_{i}\right\}_{i \in \mathbb{N}}$ que é convergente a um $x \in K$. Da mesma maneira temos que a subsequência $\left\{\omega_{i_{j}}\left(x_{i_{j}}\right)\right\}_{j \in \mathbb{N}}$ também admite uma subsequência convergente, somente por comodidade vamos supor que tal subsequência convergente de $\left\{\omega_{i_{j}}\left(x_{i_{j}}\right)\right\}_{j \in \mathbb{N}}$ é ela mesma, assim podemos supor que $\left\{\omega_{i_{j}}\left(x_{i_{j}}\right)\right\}_{j \in \mathbb{N}}$ converge a 
$y \in K$. Logo

$$
d\left(\omega_{i_{j}}(x), y\right) \leq d\left(\omega_{i_{j}}(x), \omega_{i_{j}}\left(x_{i_{j}}\right)\right)+d\left(y, \omega_{i_{j}}\left(x_{i_{j}}\right)\right)=d\left(x, x_{i_{j}}\right)+d\left(y, \omega_{i_{j}}\left(x_{i_{j}}\right)\right) .
$$

Portanto $\lim _{j \rightarrow \infty} \omega_{i_{j}}(x) \rightarrow y$. Observe que $y$ está em $\Gamma(x)$, pois, por hipótese, $\Gamma(x)$ é fechado. Agora, para cada $\omega_{i_{j}}$ existem finitos $\omega_{i_{k}}$ tais que $\omega_{i_{j}}(x)=\omega_{i_{k}}(x)$, pois $\Gamma_{x}$ é finito por hipótese. Então existe uma subsequência de $\left\{\omega_{i_{j}}(x)\right\}_{j \in \mathbb{N}}$, onde todos os pontos são diferentes, que converge para $y$, mas isso é um absurdo pois $y \in \Gamma(x)$ e $\Gamma(x)$ é discreto por hipótese. Portanto $\Gamma$ age descontinuamente sobre $Y$.

Lema 1.2.3 Seja $\Gamma$ um subgrupo de isometrias de um espaço métrico $(Y, d)$. Se existe um ponto x em $Y$ tal que $\Gamma(x)$ é discreto em $Y$ e $\Gamma_{x}$ é finito, então $\Gamma$ é um grupo discreto.

Demonstração. Seja $x$ um ponto de $Y$, e vamos supor que $\Gamma_{x}$ é finito e $\Gamma(x)$ é um subconjunto fechado e discreto de $Y$. Seja

$$
\begin{aligned}
\mu_{x}: \Gamma & \longrightarrow \Gamma(x) \\
\omega & \longmapsto \omega(x) .
\end{aligned}
$$

Dado que $\Gamma(x)$ é discreto e $\mu_{x}$ é contínua, temos que $\mu_{x}^{-1}(x)=\Gamma_{x}$ é aberto em $\Gamma$. Assim, dado que $\Gamma_{x}$ é finito por hipótese, temos que $\{e\}$, onde $e$ é o elemento identidade de $\Gamma$, é aberto em $\Gamma$. Assim, por [Rat06] (Lema 1, pág. 158), temos que $\Gamma$ é discreto.

Definição 1.2.2 Dado um espaço métrico $(Y, d)$, dizemos que $Y$ é um espaço métrico próprio se dados $y \in Y$ e $r>0$, temos que $\overline{B_{r}(y)}$ é um subconjunto compacto de $Y$.

Observação 1.2.1 Observemos que como consequência do Teorema de Hopf-Rinow, no caso de uma variedade Riemanniana $(M, \mathrm{~g})$, temos que ela é própria como espaço métrico se e somente se ela é completa. Também é bom lembrar que se $(Y, d)$ é um espaço métrico próprio, então $(Y, d)$ é um espaço métrico localmente compacto e completo (vide [dlH0o]).

Teorema 1.2.2 Sejam um espaço métrico $(Y, d)$ próprio e $\Gamma$ um grupo que age por isometrias sobre $Y$. Então $\Gamma$ é discreto se e somente se $\Gamma$ age discontinuamente sobre $Y$.

Demonstração. Primeiro vamos supor que $\Gamma$ age descontinuamente sobre $Y$. Seja $x \in M$, então, pelos Lemas 1.2.1 e 1.2.2, temos que $\Gamma_{x}$ é finito e $\Gamma(x)$ é um subconjunto fechado e discreto de $Y$, respectivamente. Logo, pelo Lema 1.2.3, temos que $\Gamma$ é discreto. 
Agora vamos supor que $\Gamma$ é discreto. Definamos $\mathcal{C}(Y, Y):=\{f: Y \rightarrow Y: f$ é contínua $\}$. Agora, dado que $Y$ é próprio, temos que $Y$ possui uma base enumerável, assim o conjunto $\mathcal{C}(Y, Y)$ possui uma base enumerável. Mais ainda, $\mathcal{C}(Y, Y)$ é regular dado que $Y$ é regular. Portanto $\mathcal{C}(Y, Y)$ é metrizável. Por [Rat06] (Lema 6, pág. 163) temos que Iso $(Y, d)$ é fechado em $\mathcal{C}(Y, Y)$ e, dado que $\Gamma$ é discreto e por [Rat06] (Lema 3 , pág. 157), temos que $\Gamma$ é fechado em Iso $(Y, d)$. Portanto $\Gamma$ é fechado em $\mathcal{C}(Y, Y)$.

Seja $K$ um subconjunto compacto de $Y$, e definamos

$$
\mathcal{K}:=\{\omega \in \Gamma: \omega(K) \cap K \neq \varnothing\}
$$

Temos então que $\mathcal{K}$ é fechado em $\mathcal{C}(Y, Y)$, pois $\Gamma$ é discreto e fechado em $\mathcal{C}(Y, Y)$. Agora, para todo $x \in Y$, todo $r>0$ e $\omega \in \Gamma$, temos que $\omega\left(B_{r}(x)\right)=B_{r}(\omega(x))$, portanto $\mathcal{K}$ é uma família equicontínua. Sejam $x$ e $y$ pontos de $Y$, e definamos $r:=\operatorname{dist}(x, y)$ e $s:=\operatorname{diam}(K)$. Assim, para cada $\omega \in \Gamma$, temos que

$$
d(y, \omega(x)) \leq d(y, \omega(y))+d(\omega(y), \omega(x)) \leq 2 s+r .
$$

Portanto $\mu_{x}(\mathcal{K})=\{\omega(x): \omega \in \mathcal{K}\} \subset B_{2 s+r}(y)$, e assim $\overline{\mu_{x}(\mathcal{K})}$ é compacto em $Y$. Aplicando o Teorema de Arzelà-Ascoli obtemos que $\mathcal{K}$ é relativamente compacto em $\mathcal{C}(Y, Y)$, i.e., $\overline{\mathcal{K}}$ é compacto em $\mathcal{C}(Y, Y)$. Por hipótese, $\Gamma$ é discreto, logo $\mathcal{K}$ é compacto, e portanto é finito. Então $\Gamma$ age descontinuamente.

Observação 1.2.2 Observe que no caso em que $Y$ seja uma variedade Riemanniana completa $(M, \mathrm{~g})$, então temos que o Teorema 1.2.2 é válido. Também observemos que não precisamos que o espaço métrico $Y$ seja próprio para ter a primeira parte do resultado do Teorema 1.2.2, i.e., se $\Gamma$ age descontinuamente por isometrias sobre $Y$, então $\Gamma$ é discreto.

Definição 1.2.3 Seja $\Gamma$ um grupo agindo sobre um espaço métrico $(Y, d)$. Definimos o espaço de $\Gamma$-órbitas ou, simplesmente, o espaço de órbitas como o seguinte conjunto

$$
Y / \Gamma:=\{\Gamma(x): x \in Y\}
$$


E definimos a aplicação quociente como

$$
\begin{aligned}
\pi: Y & \longrightarrow Y / \Gamma \\
x & \longmapsto \Gamma(x)
\end{aligned}
$$

Dotaremos o espaço $Y / \Gamma$ com a topologia quociente, i.e., $U \subset Y / \Gamma$ é aberto se e somente se $\pi^{-1}(U)$ é aberto em $Y$, e neste caso o espaço será denotado por $\left(Y / \Gamma, \tau_{\mathcal{Q}}\right)$ e será chamado espaço quociente.

Sobre o espaço de órbitas definimos a seguinte função distância

$$
\begin{array}{rlcc}
d_{\Gamma}: & Y / \Gamma \times Y / \Gamma & \longrightarrow & \mathbb{R} \\
(\Gamma(x), \Gamma(y)) & \longmapsto & \operatorname{dist}(\Gamma(x), \Gamma(y)) .
\end{array}
$$

Onde dist $(\Gamma(x), \Gamma(y)):=\inf \{d(x, \omega(y)): \omega \in \Gamma\}$. Se $d_{\Gamma}$ é uma métrica sobre $Y / \Gamma$, então diremos que $\left(Y / \Gamma, d_{\Gamma}\right)$ é o espaço de órbitas métrico. Neste caso, se $d_{\Gamma}$ é uma métrica, denotaremos a topologia em $Y / \Gamma$ gerada pela métrica $d_{\Gamma}$ por $\tau_{\Gamma}$, e portanto $\left(Y / \Gamma, \tau_{\Gamma}\right)$ é um espaço topológico metrizável.

Teorema 1.2.3 Seja $\Gamma$ um subgrupo de isometrias de um espaço métrico $(Y, d)$. Então $d_{\Gamma}$ é uma métrica sobre $Y / \Gamma$ se e somente se cada órbita é um subconjunto fechado de $Y$.

Demonstração. Sejam $x, y \in Y, \omega_{1}, \omega_{2} \in \Gamma$. Então

$$
d\left(\omega_{1}(x), \omega_{2}(y)\right)=d\left(x, \omega_{1}^{-1} \omega_{2}(y)\right) .
$$

Logo

$$
\operatorname{dist}(\Gamma(x), \Gamma(y))=\operatorname{dist}(x, \Gamma(y)) .
$$

Primeiro vamos supor que $d_{\Gamma}$ é uma métrica em $Y / \Gamma$. Sejam $x, y \in Y$ tais que $\Gamma(y) \neq \Gamma(x)$. Então

$$
\operatorname{dist}(x, \Gamma(y))=d_{\Gamma}(\Gamma(x), \Gamma(y))>0 .
$$

Assim, definamos $r:=\operatorname{dist}(x, \Gamma(y))$. Então $B_{r}(x) \subset Y \backslash \Gamma(y)$. Portanto $Y \backslash \Gamma(y)$ é aberto em $Y$, e $\operatorname{assim} \Gamma(y)$ é fechado em $Y$.

Vamos provar a recíproca. Suponhamos que cada $\Gamma$-órbita é fechada em $Y$. Queremos provar que $d_{\Gamma}$ é uma métrica em $Y / \Gamma$. 
1. Vamor ver que $d_{\Gamma}$ é não degenerada. Sejam $x, y$ em $Y$ tais que $\Gamma(x) \neq \Gamma(y)$. Em particular, $x \notin \Gamma(y)$. Dado que, por hipótese, $\Gamma(y)$ é fechado, temos que dist $(x, \Gamma(y))>0$. Logo temos que $d_{\Gamma}$ é não degenerada.

2. É fácil ver que $d_{\Gamma}$ é simétrica.

3. Provemos a desigualdade triangular. Sejam $x, y, z \in Y$ e $\omega_{1}, \omega_{2} \in \Gamma$, então

$$
\begin{aligned}
d\left(x, \omega_{1}(y)\right)+d\left(y, \omega_{2}(z)\right) & =d\left(x, \omega_{1}(y)\right)+d\left(\omega_{1}(y), \omega_{1} \omega_{2}(z)\right) \\
& \geq d\left(x, \omega_{1} \omega_{2}(z)\right) \\
& \geq \operatorname{dist}(x, \Gamma(z)) .
\end{aligned}
$$

Logo

$$
\operatorname{dist}(\Gamma(x), \Gamma(y))+\operatorname{dist}(\Gamma(y), \Gamma(z)) \geq \operatorname{dist}(\Gamma(x), \Gamma(z)) .
$$

Então temos a desigualdade triangular.

Logo $d_{\Gamma}$ é uma métrica sobre $Y / \Gamma$.

Corolário 1.2.1 Se $\Gamma$ age descontinuamente por isometrias sobre um espaço métrico $(Y, d)$, então $\left(Y / \Gamma, d_{\Gamma}\right)$ é um espaço métrico.

Demonstração. Dado que $\Gamma$ age descontinuamente sobre $Y$ temos, pelo Teorema 1.2.1, que cada Г- órbita é fechada. Logo segue-se o resultado aplicando o Teorema 1.2.3.

Teorema 1.2.4 Seja $\Gamma$ um subgrupo de Iso $(Y, d)$ tal que $\left(Y / \Gamma, d_{\Gamma}\right)$ é um espaço métrico. Então as topologias $\tau_{\Gamma}$ e $\tau_{\mathcal{Q}}$ em $Y / \Gamma$ são iguais; se $\pi: Y \rightarrow M / \Gamma$ é a aplicação quociente, então para cada $x \in Y$ e $r>0$, temos que

$$
\pi\left(B_{r}(x)\right)=B_{r}(\pi(x))
$$

Demonstração. Primeiro vamos provar que $\pi$ é aberta e contínua com a topologia $\tau_{\Gamma}$. Dados $x \in Y$ e $r>0$, é fácil ver que $\pi\left(B_{r}(x)\right) \subset B_{r}(\pi(x))$. Vamos provar a outra inclusão. Supomos que $y \in Y$ e $d_{\Gamma}(\Gamma(x), \Gamma(y))<r$. Então dist $(x, \Gamma(y))<r$. Consequentemente, existe $\omega \in \Gamma$ tal que $d(x, \omega(y))<r$. Mais ainda, temos que $\pi(\omega(y))=\Gamma(y)$, e portanto $\Gamma(y) \in \pi\left(B_{r}(x)\right)$. Assim $B_{r}(\pi(x)) \subset \pi\left(B_{r}(x)\right)$. Então $\pi$ é aberta e contínua com respeito à topologia gerada por $d_{\Gamma}$. 
Seja $U$ aberto em $\left(Y / \Gamma, \tau_{\mathcal{Q}}\right)$. Então $\pi^{-1}(U)$ é aberto em $Y$. Dado que $\pi$ é aberta e contínua com respeito à métrica $\tau_{\Gamma}$, temos que $\pi\left(\pi^{-1}(U)\right)$ é aberto em $\left(Y / \Gamma, \tau_{\Gamma}\right)$, mas $\pi\left(\pi^{-1}(U)\right)=U$. Logo $U$ é aberto em $\tau_{\Gamma}$. Assim $\tau_{\mathcal{Q}} \subset \tau_{\Gamma}$.

Agora vamos provar a outra contenção. Sejam $x \in Y$ e $r>0$. Sabemos que $B_{r}(\pi(x))$ é um aberto em $\tau_{\Gamma}$. Agora

$$
\pi^{-1}\left(B_{r}(\pi(x))\right)=\bigcup_{\omega \in \Gamma} B_{r}(\omega(x)) .
$$

Logo $B_{r}(\pi(x))$ é um aberto em $\tau_{\mathcal{Q}}$, e portanto $\tau_{\Gamma} \subset \tau_{\mathcal{Q}}$.

Assim temos que $\tau_{\Gamma}=\tau_{\mathcal{Q}}$.

Proposição 1.2.1 Seja $\Gamma$ um subgrupo de Iso $(Y, d)$. $\Gamma$ é discreto e age propriamente se e somente se $\Gamma$ age descontinuamente.

Demonstração. Primeiro vamos supor que $\Gamma$ é discreto e que age propriamente sobre $Y$. Então, por [Lee11] (Proposição 12.23) e dado que estamos supondo que $\Gamma$ é dicreto, temos que $\Gamma$ age descontinuamente sobre $Y$.

Vamos provar a recíproca. Se $\Gamma$ age descontinuamente sobre $Y$, temos, pela Observação 1.2.2, que $\Gamma$ é discreto. E o fato que $\Gamma$ age propriamente sobre $Y$ segue-se novamente de [Lee11] (Proposição 12.23).

Proposição 1.2.2 Seja $\Gamma$ um subgrupo de Iso $(Y, d)$ que age descontinuamente em $Y$. Para cada $x \in Y$ existe $r>0$, tal que

$$
\left\{\omega \in \Gamma: \omega\left(B_{r}(x)\right) \cap B_{r}(x)\right\}=\Gamma_{x} .
$$

Além disso, para para cada $y \in B_{r}(x)$, temos que $\Gamma_{y} \subset \Gamma_{x}$.

Demonstração. Seja $x \in Y$, e definamos $s:=\frac{1}{2} \operatorname{dist}(x, \Gamma(x) \backslash\{x\})$, e seja $0<r \leq s$.

Seja $\omega \in\left\{\omega \in \Gamma: \omega\left(B_{r}(x)\right) \cap B_{r}(x) \neq \varnothing\right\}$, então existe $y \in B_{r}(x)$ tal que $\omega(y) \in B_{r}(x)$. Agora

$$
d(x, \omega(x)) \leq d(x, \omega(y))+d(\omega(y), \omega(x))<2 r \leq \operatorname{dist}(x, \Gamma(x) \backslash\{x\}) .
$$

Logo $\omega \in \Gamma_{x}$. Por outra parte, é fácil ver que se $\omega \in \Gamma_{x}$, então $\omega\left(B_{r}(x)\right) \cap B_{r}(x) \neq \varnothing$. Portanto $\left\{\omega \in \Gamma: \omega\left(B_{r}(x)\right) \cap B_{r}(x)\right\}=\Gamma_{x}$, e temos a primeira parte da proposição.

Sejam $y \in B_{r}(x)$ e $\omega_{1} \in \Gamma_{y}$, então

$$
d\left(y, \omega_{1}(x)\right)=d\left(\omega_{1}^{-1}(y), x\right)=d(y, x)<r .
$$


Logo $y \in \omega_{1}\left(B_{r}(x)\right) \cap B_{r}(x)$. Aplicando a primeira parte da proposição, temos que $\omega_{1} \in \Gamma_{x}$. Assim temos a segunda parte da proposição.

Definição 1.2.4 Um espaço métrico $(Y, d)$ é chamado rígido se para cada $x \in Y$ e cada $r>0$, temos que a única isometria que fixa cada ponto de $B_{r}(x)$ é a isometria identidade, i.e., se $\omega \in$ Iso $(Y, d)$ e é tal que $\omega(y)=y$, para todo $y \in B_{r}(x)$, então $\omega=e$.

Observação 1.2.3 Se $(Y, d)$ é um espaço métrico que é geodesicamente conexo e geodésicamente completo, então $(Y, d)$ torna-se um espaço rígido (vide [Rat06], Teorema 6.6.10.). Observemos que, em especial, toda variedade Riemanniana completa é um espaço métrico rígido.

Proposição 1.2.3 Sejam $Y$ e $\Gamma$ com as mesmas hipóteses da Proposição 1.2.2. Se $Y$ é rígido, então existe um $x_{0} \in Y$ tal que $\Gamma_{x_{0}}=\{e\}$, onde e é o elemento identidade de $\Gamma$.

Demonstração. Dado que $\Gamma$ age descontinuamente, temos que $\left|\Gamma_{x}\right|$ é finito, para cada $x \in M$. Assim, seja $x_{0} \in Y$ tal que $\left|\Gamma_{x_{0}}\right| \leq\left|\Gamma_{x}\right|$ para cada $x \in Y$. Pela Proposição 1.2.2 existe $r>0$ tal que $\Gamma_{x_{0}}=\left\{\omega \in \Gamma: \omega\left(B_{r}\left(x_{0}\right)\right) \cap B_{r}\left(x_{0}\right) \neq \varnothing\right\}$ e para cada $y \in B_{r}(x)$ acontece que $\Gamma_{y} \subset \Gamma_{x_{0}}$. Assim, para cada $y \in B_{r}(x)$ temos que $\Gamma_{y}=\Gamma_{x_{0}}$, pois $\left|\Gamma_{x_{0}}\right| \leq\left|\Gamma_{x}\right|$ para cada $x \in Y$. Logo para cada $y \in B_{r}(x)$ e para cada $\omega \in \Gamma_{x}$, temos que $\omega(y)=y$, i.e., $\left.\omega\right|_{B_{r}(x)}=\left.e\right|_{B_{r}(x)}$. Portanto, dado que $Y$ é espaço métrico rígido, $\omega=e$. Logo $\Gamma_{x_{0}}=\{e\}$.

Teorema 1.2.5 Seja $\Gamma$ um subgrupo de Iso $(Y, d)$ agindo descontinuamente sobre $Y$. Então para cada $x \in Y$ a aplicação quociente $\pi: Y \rightarrow Y / \Gamma$ induz um homeomorfismo entre $B_{r}(x) / \Gamma_{x} e$ $B_{r}(x) / \Gamma$, para todo $0<r \leq \frac{1}{2} \operatorname{dist}(x, \Gamma(x) \backslash\{x\})$. Mais ainda, tal homeomorfismo torna-se uma isometria no caso em que $0<r \leq \frac{1}{4} \operatorname{dist}(x, \Gamma(x) \backslash\{x\})$.

Demonstração. Seja $x$ em $Y$. Pelo Teorema 1.2.4 temos que

$$
B_{r}(\pi(x))=\pi\left(B_{r}(x)\right)
$$

para todo $r>0$. Assim, $\pi$ é aberta. Seja $s:=\frac{1}{2} \operatorname{dist}(x, \Gamma(x) \backslash\{x\})$ e suponha que $0<r \leq s$. Seja $\omega \in \Gamma$. Sabemos, pela Proposição 1.2.2, que $\omega \in \Gamma_{x}$ se e somente se $\omega \in\left\{\bar{\omega} \in \Gamma: \bar{\omega}\left(B_{r}(x)\right) \cap\right.$ $\left.B_{r}(x) \neq \varnothing\right\}$. Assim $\pi$ induz o homeomorfimo

$$
\begin{array}{rlr}
B_{r}(x) / \Gamma_{x} & \longrightarrow & B_{r}(x) / \Gamma \\
\Gamma_{x}(y) & \longrightarrow & \Gamma(y) .
\end{array}
$$


Dado que $\Gamma_{x}$ é finito, então $\Gamma_{x}$ age descontinuamente sobre $B_{r}(x)$, para todo $0<r \leq s$. E assim temos que $\left(B_{r}(x), d_{\Gamma_{x}}\right)$ é um espaço métrico. Agora suponhamos que $0<r \leq \frac{s}{2}$. E vamos provar que o homeomorfismo dado em (1.3) é uma isometria.

Sejam $y$ e $z$ em $B_{r}(x)$. Agora, dado que $\Gamma_{x}$ é finito, podemos supor que $d(y, z)=\operatorname{dist}\left(y, \Gamma_{x}(z)\right)$. Seja $\omega \in \Gamma \backslash \Gamma_{x}$, então

$$
\begin{aligned}
2 s & \leq d((x), \omega(x)) \\
& \leq d(x, y)+d(y, \omega(z))+d(\omega(z), \omega(x)) \\
& <r+d(y, \omega(z))+r \\
& =2 r+d(y, \omega(z)) \\
& \leq s+d(y, \omega(z)) .
\end{aligned}
$$

Logo $s<d(y, \omega(z))$, e portanto $d(y, z)<2 r \leq s<d(y, \omega(z))$, para todo $\omega \in \Gamma \backslash \Gamma_{x}$. Assim, dado que $d(y, z)=\inf \left\{d(y, \omega(z)): \omega \in \Gamma_{x}\right\}$ e $d(y, z)<d(y, \omega(z))$, para todo $\omega \in \Gamma \backslash \Gamma_{x}$, temos que $d(y, z)=\inf \{d(y, \omega(z)): \omega \in \Gamma\}$. E, portanto,

$$
d_{\Gamma}(\Gamma(y), \Gamma(z))=d(y, z)=d_{\Gamma_{x}}\left(\Gamma_{x}(y), \Gamma_{x}(z)\right) .
$$

Assim, temos que o homeomorfimo definido em (1.3) é uma isometria.

\subsection{Domínios Fundamentais}

Definição 1.3.1 Sejam $Y$ um espaço métrico e $\Gamma$ um subconjunto de Iso $(Y, d)$ que age sobre $Y$. Um subconjunto $R \subset Y$ é chamado uma região fundamental para $\Gamma$ se e somente se

1. $R$ é um subconjunto aberto de $Y$;

2. a família $\{\omega(R): \omega \in \Gamma\}$ é disjunta dois a dois;

3. $M=\bigcup_{\omega \in \Gamma} \omega(\bar{R})$.

$E$ dizemos que $D \subset Y$ é um dominio fundamental para $\Gamma$, se $D$ é conexo e é uma região fundamental para $\Gamma$

Observação 1.3.1 Obsermos que se $R$ é uma região fundamental para $\Gamma$, então para cada y $\in R$ vamos ter que $\Gamma_{y}=\{e\}$. Assim, temos que nem toda ação descontínua admite a existência de uma região fundamental. Olhemos o seguinte exemplo. 
Exemplo 1.3.1 Seja $Y$ a cruz em $\mathbb{R}^{2}$, i.e., a união do eixo-x e do eixo-y. Sejam $\rho_{x}: \mathbb{R}^{2} \rightarrow \mathbb{R}^{2}$, definida como $\rho_{x}(x, y)=(x,-y)$ e $\rho_{y}: \mathbb{R}^{2} \rightarrow \mathbb{R}^{2}$, definida como $\rho_{x}(x, y)=(-x, y)$. Definamos $\Gamma:=\left\langle\rho_{x}, \rho_{y}\right\rangle$. Assim $\Gamma=\left\{\rho_{x}, \rho_{y}, e,-e\right\}$, e portanto é finito. Agora, $\Gamma$ age descontinuamente e por isometrias sobre $Y$, as nenhum ponto de $Y$ tem grupo de isotropia trivial. Assim, pela Observação 1.3.1, temos que $\Gamma$ no possui região fundamental.

O seguinte teorema será útil no Capítulo 3. Vide [Rat06] (Teorema 6.6.9) para uma prova dele.

Teorema 1.3.1 Sejam $(Y, d)$ um espaço métrico localmente compacto e $\Gamma$ um subgrupo de Iso $(Y, d)$ que age descontinuamente sobre $Y$ e tal que $Y / \Gamma$ é compacto. Seja $R$ uma região fundamental para a ação de $\Gamma$ sobre $Y$, então $R$ é uma região fundamental localmente finita se, e somente se, $\bar{R}$ é compacto.

Definição 1.3.2 Sejam $Y$ um espaço métrico e $\Gamma$ um subgrupo de Iso $(Y, d)$ agindo sobre $Y$. Vamos supor que existe um ponto $y \in Y$ tal que $\Gamma_{y}$ é trivial (por exemplo, quando $Y$ é rígido ou é uma variedade Riemanniana (vide a Proposição 1.2.3)). Dado $\omega \in \Gamma$, definamos

$$
H_{\omega}(y):=\{x \in Y: d(y, x)<d(y, \omega(x))\} .
$$

Observação 1.3.2 Afirmamos que $H_{\omega}(y)$ é um sunconjunto aberto de $Y$. Com efeito, seja $\left\{x_{i}\right\}_{i \in \mathbb{N}}$ uma sequência no complemento de $H_{\omega}(y)$ e supomos que $\lim _{i \rightarrow \infty} x_{i} \rightarrow x_{0}$. Assim

$$
d\left(y, x_{0}\right)=\lim _{i \rightarrow \infty} d\left(y, x_{i}\right) \geq d\left(y, \omega\left(x_{i}\right)\right)=d\left(y, \omega\left(x_{0}\right)\right) .
$$

Portanto, $x_{0}$ é um ponto no complemento de $H_{\omega}(y)$. E assim está provada a afirmação.

Definição 1.3.3 Sejam $Y$ um espaço métrico e $\Gamma$ um subgrupo de isometrias de $Y$. Vamos supor que $\Gamma$ age descontínuamente sobre $Y$ e que existe um ponto $y \in Y$ tal que $\Gamma_{y}$ é trivial. Definamos o dominio de Dirichlet para $\Gamma$ com centro em $y$, como

$$
D(y):=\bigcap_{\omega \in \Gamma \backslash\{e\}} H_{\omega}(y) .
$$

O seguinte resultado será muito útil no Capítulo 3, e a prova deste pode ser vista em [Rat06].

Teorema 1.3.2 Sejam $Y$ um espaço métrico e $\Gamma$ um subconjunto de isometrias de $Y$. Vamos supor que $\Gamma$ age descontinuamente sobre $Y$. Seja $D(y)$ um domínio de Dirichlet com centro em y. Supomos que $Y$ atende as seguintes propriedades 
1. Y é geodesicamente completo;

2. $Y$ é geodesicamente conexo;

3. $Y$ é próprio.

Então $D(y)$ é um dominio fundamental para $\Gamma$. Além disso, a familia $\{\omega(D(x)): \omega \in \Gamma\}$ é localmente finita $e$

$$
\overline{D(y)}=\{x \in Y: d(y, x)=d(y, \Gamma(x))\} .
$$

Demonstração. Vide [Rat06] (Teorema 6.6.13 e Teorema 6.6.14).

\subsection{Orbifolds}

Considere uma variedade Riemanniana simplesmente conexa $(M, \mathrm{~g})$ e $\Gamma$ um subgrupo discreto de Iso $(M, \mathrm{~g})$ agindo propriamente sobre $M$. Observe que neste caso, pela Proposição 1.2.2, $\Gamma$ age descontinuamente sobre $M$, e então, por todos os resultados apresentados na seção anterior, vamos ter que o objeto $M / \Gamma$ possui propriedades importantes, e é por isso que $M / \Gamma$ será nosso objeto de estudo, e será chamado good orbifold Riemanniano.

Definição 1.4.1 Seja X uma espaço topológico Hausdorff. Uma estrutura orbifold diferenciável sobre $X$ é dada pelos seguintes dados

1. Uma cobertura por abertos $\left\{U_{i}\right\}_{i \in I}$ de $X$.

2. Para cada $i \in I$, existem uma n-variedade diferenciável simplesmente conexa $M_{i}$, um subgrupo discreto $\Gamma_{i} \in \operatorname{Dif}(M)$ que age propriamente e efetivamente sobre $M_{i}$ e uma aplicação contínua, sobrejetora e $\Gamma_{i}$ - invariante, $\bar{\phi}_{i}: M_{i} \rightarrow U_{i}$, chamada carta uniformizadora, que induz um homeomorfimo $\phi_{i}: M_{i} / \Gamma_{i} \rightarrow U_{i}$.

3. Se $x_{i} \in M_{i}$ e $x_{j} \in M_{j}$ são tais que $\bar{\phi}_{i}\left(x_{i}\right)=\bar{\phi}_{j}\left(x_{j}\right)$, então existe vizinhanças abertas $A_{i}$ e $B_{j}$ de $x_{i}$ e $x_{j}$, respectivamente, e um difeomorfismo $h_{i j}: A_{i} \rightarrow B_{j}$ tal que $\left.\bar{\phi}_{i}\right|_{A_{i}}=\bar{\phi}_{j} \circ h_{i j}$. Temos que $h_{i j}$ está bem definida salvo composição com um elemento de $\Gamma_{j}$. Em particular, se $i=j$, então $h_{i j}=\left.\omega\right|_{A_{i}}$, onde $\omega$ é um elemento de $\Gamma_{i}$. Os difeomorfimos locais $h_{i j}$ são chamados difeomorfimos de mudança de cartas.

Assim, a família $\left\{\left(U_{i}, M_{i}, \bar{\phi}_{i}, \Gamma_{i}\right)\right\}_{i \in I}$ é chamada um atlas para a estrutura orbifold diferenciável sobre $X$. 
Definição 1.4.2 Dois atlas $\left\{\left(U_{i}, \bar{\phi}_{i}, \Gamma_{i}\right)\right\}_{i \in I}$ e $\left\{\left(V_{j}, \bar{\phi}_{j}, \Gamma_{j}\right)\right\}_{j \in J}$ definem a mesma estrutura para $X$ se a familia $\left\{\left(W_{k}, \bar{\varphi}_{k}, \Gamma_{k}\right)\right\}_{k \in I \cup J}$ satisfaz a condição de compatibilidade (item número 3 da definição anterior), onde $W_{k} \in\left\{U_{i}, V_{j}: i \in I, j \in J\right\}, \bar{\varphi}_{k} \in\left\{\bar{\phi}_{i}, \bar{\phi}_{j}: i \in I, j \in J\right\}$ e $\Gamma_{k} \in\left\{\Gamma_{i}, \Gamma_{j}: i \in I, j \in J\right\} . E$ dizemos que $X$ é um orbifold diferenciável se ele possui uma estrutura orbifold diferenciável maximal.

Proposição 1.4.1 Se $X$ admite uma estrutura orbifold diferenciável, então $X$ é é um orbifold diferenciável, i.e., toda estrutura orbifold diferenciável está contida numa estrutura orbifold diferenciável maximal.

Observação 1.4.1 Se $X$ é um orbifold diferenciável e $\left\{\left(U_{i}, \bar{\phi}_{i}, \Gamma_{i}\right)\right\}_{i \in I}$ é uma estrutura para $X$ tal que os grupos $\Gamma_{i}$ são triviais ou, mais geralmente, se eles agem livremente sobre as variedades $M_{i}$, respectivamente, então $X$ é uma variedade diferenciável.

Também podemos observar que se os grupos $\Gamma_{i}$ são finitos, então a ação deles sobre as variedades $M_{i}$ é sempre efetiva.

Proposição 1.4.2 Sejam $M$ uma variedade diferenciável conexa e $\Gamma$ um subgrupo discreto de Dif( $M)$ que age propriamente sobre $M$. Então $M / \Gamma$ com a topologia quociente possui uma estrutura orbifold diferenciável.

Demonstração. Temos que, dado que $\Gamma$ age propriamente sobre $M$, o espaço $M / \Gamma$ com a topologia quociente é espaço topológico Hausdorff (vide Proposição 1.1.3). Agora, seja $\pi: M \rightarrow M / \Gamma$ a aplicação quociente. Logo, dado que $\Gamma$ é um subgrupo discreto de $\operatorname{Dif}(M), \pi$ é uma aplicação aberta quando munimos o espaço $M / \Gamma$ com a topologia quociente. Agora, pela Proposição 1.1.3, para cada $x \in M$ existe uma vizinhança aberta $V_{x} \subset M$ contendo $x$ e tal que

$$
\left\{\omega \in \Gamma: \omega\left(V_{x}\right) \cap V_{x} \neq \varnothing\right\}=\Gamma_{x} .
$$

Sem perda de geralidade podemos supor que cada $V_{x}$ é simplesmente conexo e que $\left\{V_{x}\right\}_{x \in M}$ é uma cobertura por abertos, onde os $V_{x}$ são abertos de um atlas maximal para $M$. Observemos que $\Gamma_{x}$ é finito e age própria e efetivamente sobre $V_{x}$. E também temos que $\pi$ induz um homeomorfimo

$$
\begin{aligned}
V_{x} / \Gamma_{x} & \longrightarrow V_{x} / \Gamma \\
\Gamma_{x}(y) & \longmapsto \Gamma(y)
\end{aligned}
$$

Assim, afirmamos que a família $\left\{\left(V_{x},\left.\pi\right|_{V_{x}}, \Gamma_{x}\right)\right\}_{x \in M}$ é uma estrutura orbifold diferenciável para $M / \Gamma$. 
Definindo $U_{x}:=\pi\left(V_{x}\right)$, a família $\left\{U_{x}\right\}_{x \in M}$ é uma cobertura por abertos de $M / \Gamma$. Assim temos a primeira condição da definição 1.4.1. Agora, temos que $\left.\pi\right|_{V_{x}}: V_{x} \rightarrow U_{x}$ induz um homeomorfimo entre $V / \Gamma_{x}$ e $U_{x}$, dado por 1.4. Logo temos a segunda condição da Definição 1.4.1. Sejam $x$ e $y \in M$ tais que $\pi(x)=\pi(y)$, então existe $\omega \in \Gamma$ tal que $\omega(x)=y$. Agora, dado que $\omega$ é um difeomorfimo e $V_{y}$ é um aberto, temos que existe um aberto $x \in A_{x} \subset V_{x}$ tal que $\omega\left(A_{x}\right) \subset V_{y}$. Portanto $\left.\omega\right|_{A_{x}}: W_{x} \rightarrow \omega\left(A_{x}\right)$ é um difeomorfismo e $\left.\pi \circ \omega\right|_{A_{x}}=\left.\pi\right|_{A_{x}}$. Assim temos a condição 3 da definição 1.4.1.

Definição 1.4.3 Um orbifold diferenciável é chamado good orbifold diferenciável se ele surge como o quociente global de uma variedade diferenciável conexa por um subgrupo discreto de difeomorfimos que age propriamente sobre ela. Se um orbifold diferenciável não é um good orbifold. então é chamado simplesmente como um orbifold diferenciável.

Exemplos 1.4.1 1. Sejam $M=\mathbb{S}^{2}$ e $\Gamma$ o subgrupo de difeomorfirmos de $M$ gerado por $\omega$, onde $\omega$ é uma rotação em torno do eixo z com um ângulo de $\frac{\pi}{2}$. Neste caso temos que $\Gamma=\left\{\omega, \omega^{2}, \omega^{3}, e\right\}$, onde e é o difeomorfismo identidade de M. Dado que $\Gamma$ é finito, temos que ele é discreto e age propriamente sobre $M$, e também age efetivamente. Assim, $M / \Gamma$ é um good orbifold diferenciável.

2. Sejam $M=\mathbb{R}^{2}$ e $\tilde{\Gamma}$ o subgrupo de Dif $\left(\mathbb{R}^{2}\right)$ gerado por três reflexões $\left\{\omega_{1}, \omega_{2}, \omega_{3}\right\}$; onde $\omega_{1}$ é a reflexão em torno da reta $y=0, \omega_{2}$ é a reflexão em torno da reta $y=\sqrt{3} x$ e $\omega_{3}$ é a em reflexão em torno da reta $y=-\sqrt{3} x+\sqrt{3}$. Não é difícil ver que $\tilde{\Gamma}$ tem cardinal infinito. Além disso, para $x$ em $\mathbb{R}^{2}$ temos que $\tilde{\Gamma}_{x}$ é finito e $\tilde{\Gamma}(x)$ é subconjunto fechado e discreto de $\mathbb{R}^{2}$. Observemos que se sobre $\mathbb{R}^{2}$ temos a métrica Riemanniana canônica, então $\tilde{\Gamma}$ é um subgrupo de isometrias. Assim, pelo Teorema 1.2.1, temos que $\tilde{\Gamma}$ age descontinuamente sobre $\mathbb{R}^{2}$. Então, pela Proposição 1.2.1, temos que $\tilde{\Gamma}$ é discreto e age propriamente. Além disso, pela Proposição 1.2.3, $\Gamma$ age efetivamente. Logo $\mathbb{R}^{2} / \tilde{\Gamma}$ é um good orbifold diferenciável. Observe que $\mathbb{R}^{2} / \tilde{\Gamma}$ é homeomorfo a um triângulo equilátero em $\mathbb{R}^{2}$ de perímetro 3. 


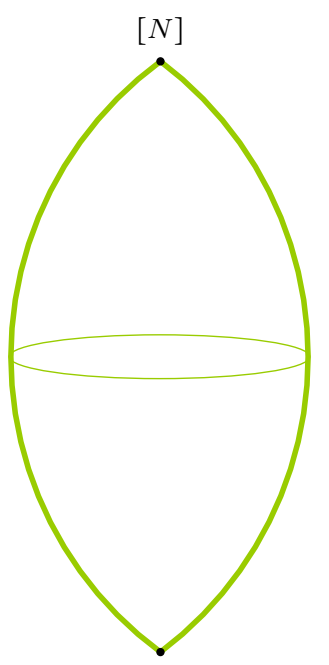

$[S]$

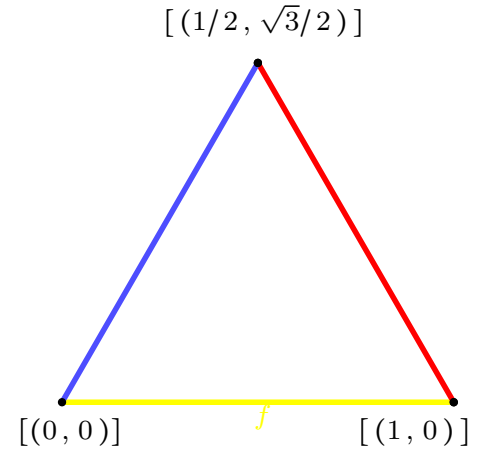

Figura 1.1: Os espaços $\mathbb{S}^{2} / \Gamma$ e $\mathbb{R}^{2} / \tilde{\Gamma}$.

\subsubsection{Orbifold Riemanniano}

Definição 1.4.4 Sejam X é um orbifold diferenciável e $\left\{\left(M_{i}, \bar{\phi}_{i}, \Gamma_{i}\right)\right\}_{i \in I}$ um atlas maximal. Dizemos que $X$ é um orbifold Riemanniano se as variedades diferenciáveis $M_{i}$ são variedades Riemannianas $\left(M_{i}, \mathrm{~g}_{i}\right)$, os subgrupos $\Gamma_{i}$, são subgrupos de Iso $\left(M_{i}, \mathrm{~g}_{i}\right)$ e os difeomorfismos locais $h_{i j}$ de mudança de cartas são isometrias locais.

Observação 1.4.2 Observe que neste caso, quando X é um orbifold Riemanniano, para cada $i \in I$, temos que, dado que $\Gamma_{i}$ é discreto e age propriamente por isometrias sobre a variedade Riemanniana $\left(M_{i}, \mathrm{~g}_{i}\right)$ e usando as Proposiçôes 1.2.1 e 1.2.3, $\Gamma_{i}$ age descontinuamente e efetivamente sobre $M$.

Proposição 1.4.3 Todo orbifold diferenciável e paracompacto $X$ admite uma estrutura de orbifold Riemanniano.

Demonstração. Vamos fazer a prova no caso no qual $X$ é um good orbifold diferenciável. Uma prova do caso em geral, pode ser vista em [Dra11].

Primeiro vamos observar que o espaço $X=M / \Gamma$ com a topologia quociente é paracompacto pois é um espaço Hausdorff localmente compacto e é a união de uma família enumerável de espaços compactos. Assim, existe uma cobertura localmente finita de abertos $\left\{\pi\left(V_{x}\right)\right\}_{x \in M}$ de $M / \Gamma$, onde cada aberto $x \in V_{x}$ é tal que $\left\{\omega \in \Gamma: \omega\left(V_{x}\right) \cap V_{x} \neq \varnothing\right\}=\Gamma_{x}$ (vide a prova da Proposição 1.4.3). Definamos

$$
\tilde{V}_{x}:=\pi^{-1}\left(\pi\left(V_{x}\right)\right)=\bigcup_{\omega \in \Gamma} \omega\left(V_{x}\right)
$$


Assim, cada $\tilde{V}_{x}$ é aberto em $M$ e é $\Gamma$-invariante. Agora seja $\left\{f_{x_{i}}\right\}_{x \in M}$ uma $\Gamma$-invariante partição da unidade subordinada à família $\left\{\tilde{V}_{x}\right\}_{x \in M}$. Tal partição existe pelo seguinte fato (para uma melhor consulta vide $[\mathrm{AB} 15])$

Se $\left\{\tilde{V}_{x}\right\}_{x \in M}$ é uma família localmente finita de abertos $\Gamma$-invariantes cobrindo $M$, então existe uma partição da unidade $\left\{f_{x}\right\}_{x \in M}$ subordinada à familia $\left\{\tilde{V}_{x}\right\}_{x \in M}$, tal que cada $f_{x}: \tilde{V}_{x} \rightarrow[0,1]$ é $\Gamma$ invariante.

Continuando com a prova, afirmamos que para cada $V_{x}$ existe uma métrica Riemanniana $\mathrm{g}^{x}$ tal que $\Gamma_{x}$ é um subgrupo de Iso $\left(V_{x}, \mathrm{~g}^{x}\right)$. Com efeito, dado que $V_{x} \subset M$, então $V_{x}$ é paracompacto, logo existe uma métrica Riemanniana g em $V_{x}$. Agora, para cada $y \in V_{x}$ e cada par $u, v \in T_{y} M$, definamos

$$
\mathrm{g}^{x}(u, v)_{y}:=\frac{1}{\left|\Gamma_{x}\right|} \sum_{\omega \in \Gamma_{x}} \mathrm{~g}\left((d \omega)_{y} u,(d \omega)_{y} v\right)_{\omega(y)} .
$$

É fácil ver que $\mathrm{g}^{x}$ é $\Gamma_{x}$-invariante, e portanto $\Gamma_{x}$ é um subgrupo de Iso $\left(V_{x}, \mathrm{~g}^{x}\right)$.

Finalmente definamos a seguinte métrica Riemanniana sobre $M$

$$
\mathrm{g}:=\sum_{x \in M} f_{x} \mathrm{~g}^{x}
$$

Exemplo 1.4.1 Os exemplos expostos em 1.4.1 são good orbifolds Riemannianos. Mais adiante vamos dar outro exemplo de um orbifold Riemaniano que não é de tipo good.

Proposição 1.4.4 Sejam ( $M, \mathrm{~g})$ uma variedade Riemanniana conexa e $\Gamma$ um subgrupo discreto de Iso $(M, \mathrm{~g})$ que age propriamente sobre $M$, então $M / \Gamma$ é um orbifold Riemanniano. Além disso $\left(M / \Gamma, \tau_{\mathcal{Q}}\right)$ é um espaço topológico metrizável.

Demonstração. A primeira parte da prova é obtida a partir da Proposição 1.4.3. A segunda parte segue-se do Corolário 1.2.1 e do Teorema 1.2.4.

\subsection{Orbifold de Recobrimento}

Definição 1.5.1 Sejam $X$ e $X^{\prime}$ dois orbifold diferenciáveis. Uma aplicação contínua e sobrejetora

$$
p: X^{\prime} \rightarrow X
$$


é chamada uma aplicação recobridora se e somente se satisfaz a seguinte condição: para cada $\bar{x} \in X$ existe uma vizinhança aberta $\bar{x} \in U_{\bar{x}} \subset X$ e uma carta orbifold $(M, \bar{\phi}, \Gamma)$ que uniformiza $U_{\bar{x}}$, i.e, $\bar{\phi}: M \rightarrow U_{\bar{x}}$ é contínua e $\Gamma$-invariante e induz um homeomorfismo $\phi: M / \Gamma \rightarrow U_{\bar{x}}$, tais que cada componente conexa $V_{i}$ de $p^{-1}\left(U_{\bar{x}}\right) \subset X^{\prime}$ é uniformizada por $\left(M, \bar{\phi}_{i}, \Gamma_{i}\right)$, onde $\Gamma_{i}$ é um subgrupo de $\Gamma$ e $\bar{\phi}_{i}: M \rightarrow V_{i}$ é contínua, sobrejetora $e \Gamma_{i}$-invariante e induz o homeomorfismo $\phi_{i}: M / \Gamma_{i} \rightarrow V_{i}$.

Observação 1.5.1 É bom observar que, geralmente, $X^{\prime}$ não é um espaço recobridor de $X$.

Definição 1.5.2 O recobrimento universal de $p: \tilde{X} \rightarrow X$ de um orbifold conexo $X$ é uma aplicação recobridora tal que se $p^{\prime}: X^{\prime} \rightarrow X$ é outra aplicação recobridora, então existe uma aplicação recobridora $\tilde{p}: \tilde{X} \rightarrow X^{\prime}$ tal que $p=p^{\prime} \circ \tilde{p}$. Neste caso $\tilde{X}$ é chamado recobridor orbifold universal de $X$.

Observação 1.5.2 William Thurston provou que cada orbifold diferenciável X possui um recobridor orbifold universal $\tilde{X}$ (vide [P.02]). Além disso, ele definiu o conceito de grupo fundamental orbifold de $X$ e é denotado por $\pi_{1}^{\text {orb }}(X)$, e é o grupo de transformações de Deck associadas à aplicação de recobrimento universal $p: \tilde{X} \rightarrow X$, i.e.,

$$
\pi_{1}^{o r b}(X):=\{\psi: \tilde{X} \rightarrow \tilde{X} \mid p=p \circ \psi\}
$$

No caso no qual $X=M / \Gamma$ é um good orbifold, temos que $\pi: M \rightarrow M / \Gamma$ é uma aplicação recobridora. Além disso, cada subgrupo $\Gamma_{0} \subset \Gamma$ induz um recobrimento $\pi_{0}: M \Gamma_{0} \rightarrow M / \Gamma$. Por outra parte, se $N$ é o variedade recobridora de $M$ e $f: N \rightarrow M$ é a aplicação recobridora respectiva, então $\pi \circ f$ : $N \rightarrow M / \Gamma$ torna-se um recobrimento orbifold. Em particular, se $N=\tilde{M}$, onde $\tilde{M}$ é o recobridor universal de $M$, então $\tilde{M}$ é o recobridor universal orbifold de $M / \Gamma$ e $\pi \circ f$ é um recobrimento universal. Assim, obtemos a seguinte sequência exata curta

$$
\{1\} \longrightarrow \pi_{1}(M) \longrightarrow \pi_{1}^{o r b}(M / \Gamma) \longrightarrow \Gamma \longrightarrow\{1\}
$$

Com efeito, sabemos que

$$
\pi_{1}(M)=\{g: \tilde{M} \rightarrow \tilde{M}: f \circ g=f\} .
$$

e por definição

$$
\pi_{1}^{\text {orb }}(M / \Gamma)=\{\psi: \tilde{M} \rightarrow \tilde{M}: \pi \circ f=\pi \circ f \circ \psi\} .
$$

Agora seja $g \in \pi_{1}(M)$, então $(\pi \circ f) \circ g=\pi \circ(f \circ g)=\pi \circ f$. Logo temos a primeira parte de sequência exata. Observe que se $\omega \in \Gamma$, então $\omega$ induz uma difeomorfimo $\psi: \tilde{M} \rightarrow \tilde{M}$, tal que 
$f \circ \psi=\omega \circ f$. Logo

$$
(\pi \circ f) \circ \psi=\pi \circ(f \circ \psi)=\pi \circ(\omega \circ f)=(\pi \circ \omega) \circ f=\pi \circ f .
$$

Portanto $\psi$ é um elemento de $\pi_{1}^{o r b}(M / \Gamma)$, e assim temos a segunda parte da sequência curta.

Observe que um orbifold diferenciável $X$ é um good orbifold se e somente se $\tilde{X}$ é uma variedade diferenciável.

Exemplo 1.5.1 Sejam $M=\mathbb{S}^{2}$ e $\Gamma$ o grupo gerado pelo seguinte difeomorfismo

$$
\begin{aligned}
& \omega_{1}: \mathbb{S}^{2} \quad \longrightarrow \quad \mathbb{S}^{2} \\
& (x, y, z) \longmapsto(x, y,-z)
\end{aligned}
$$

Então temos que $\pi_{1}^{\text {orb }}(M / \Gamma)=\mathbb{Z}_{2}$. Mas observemos que $\pi_{1}(M / \Gamma)=\{e\}$.

Este exemplo mostra que, geralmente, $\pi_{1}(M / \Gamma) \neq \pi_{1}^{\text {orb }}(M / \Gamma)$.

Antes de dar o seguinte exemplo, vamos citar um resultado muito importante (vide [Dra11], Teorema $2.21)$.

Seja X um orbifold diferenciável conexo. Sejam $X_{1}$ e $X_{2}$ dois suborbifolds conexos de $X$ e tais que

1. $X=X_{1} \cup X_{2}$;

2. $X_{3}:=X_{1} \cap X_{2}$ é conexo;

3. $\bar{X}_{1}$ e $\bar{X}_{2}$ são suborbifolds de $X$ com bordo e tais que o bordo de $X_{1}$ e $X_{2}$, respectivamente, é igual à fronteira de $X_{1}$ e $X_{2}$ em $X$, respectivamente.

Então

$$
\pi_{1}^{o r b}(X)=\pi_{1}^{o r b}\left(X_{1}\right) \star_{\pi_{1}^{o r b}\left(X_{3}\right)} \pi_{1}^{o r b}\left(X_{2}\right)
$$

é o produto livre amalgamado dos grupos fundamentais orbifold de $X_{1}$ e de $X_{2}$ sobre o grupo fundamental orbifold de $X_{3}$.

Exemplo 1.5.2 (A Gota) Seja $k \in\{2,3, \ldots\}$, definamos $\omega$ como uma rotação de um ângulo $\frac{2 \pi}{k}$ em $\mathbb{R}^{2}$. Sejam $M_{1}=M_{2}=B_{\frac{3}{4} \pi}(0)$ a bola aberta em $\mathbb{R}^{2}$ e centrada na origem de raio $\frac{3 \pi}{4}$ e $\Gamma$ o grupo gerado por $\omega$. Sejam os homeomorfismos

$$
\begin{aligned}
\phi_{1}: B_{\frac{3}{4} \pi}(0) / \Gamma & \longrightarrow U_{1} \\
{[v] } & \longmapsto \exp _{N}(v) .
\end{aligned}
$$




$$
\begin{aligned}
\bar{\phi}_{2}: B_{\frac{3}{4} \pi}(0) & \longrightarrow U_{2} \\
w & \longmapsto \exp _{S}(w) .
\end{aligned}
$$

Onde $N$ e $S$ são os polos na esfera $\mathbb{S}^{2}$ e exp $\exp _{N}, \exp ^{2} \rightarrow \mathbb{S}^{2}$ são as aplicaçôes exponencial de $N$ e $S$, respectivamente.

Observe que $\phi_{1}$ induz uma aplicação $\bar{\phi}_{1}: B_{\frac{3}{4} \pi}(0) \longrightarrow U_{1}$, que é continua, sobrejetora e $\Gamma$-invariante. Definamos $\Gamma_{1}:=\Gamma$ e $\Gamma_{2}:=\{e\}$, onde e é o difeomorfismo identidade em $B_{\frac{3}{4} \pi}(0)$. Então temos que

$$
\left\{\left(M_{i}, \bar{\phi}_{i}, \Gamma_{i}\right)\right\}_{i=1,2}
$$

define uma estrutura orbifold diferenciável para $X$. Neste caso os difeomorfismos de mudança é o grupo $\Gamma_{1}$.

Agora

$$
\begin{aligned}
\pi_{1}^{\text {orb }}(X) & =\pi_{1}^{\text {orb }}\left(U_{1}\right) \star_{\pi_{1}^{\text {orb }}\left(U_{1} \cap U_{2}\right)} \pi_{1}^{\text {orb }}\left(U_{2}\right) \\
& =\mathbb{Z}_{k} \star_{\mathbb{Z}}\{e\} . \\
& =\{e\}
\end{aligned}
$$

Portanto, pela Observação 1.5.2, temos que $\tilde{X}=X$, que não é uma variedade. Logo X não é um good orbifold.

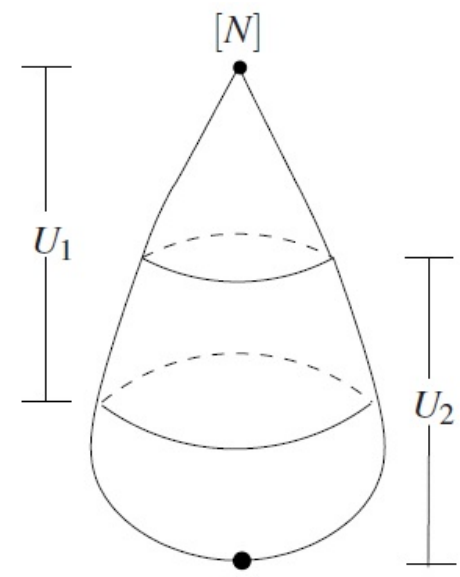

$[S]$

Figura 1.2: A Gota

\subsection{O Pseudogrupo de Mudança de Cartas}

Definição 1.6.1 Seja $M$ uma variedade Riemanniana, não necesariamente conexa. Um pseudogrupo $\mathcal{P}$ de difeomorfismos (isometrias) de $M$ é uma família de difeomorfismo (isometrias) 
locais $\omega: U \rightarrow V$, onde $U$ e $V$ são abertos de $M$, que satisfaz

1. Se $\omega \in \mathcal{P}$, entãa $\omega^{-1} \in \mathcal{P}$;

2. se $\omega_{1}: U_{1} \rightarrow V_{1}$ e $\omega_{2}: U_{2} \rightarrow V_{2}$ são elementos de $\mathcal{P}$, então $\omega_{2} \circ \omega_{1}: \omega_{1}^{-1}\left(U_{2} \cap V_{1}\right) \rightarrow V_{2}$ é um elemento de $\mathcal{P}$;

3. se $\omega: U \rightarrow V$ é um elemento de $\mathcal{P}$ e $\tilde{U}$ é um aberto contido em $U$, então $\left.\omega\right|_{\tilde{U}}: \tilde{U} \rightarrow V$ é um elemento de $\mathcal{P}$;

4. se $\omega: U \rightarrow V$ é um difeomorfimo (isometria) local em $M$ tal que para cada $p$ em $U$ existem um aberto $p \in \tilde{U} \subset U$ e $\tilde{\omega}$ em $\mathcal{P}$ tais que $\left.\omega\right|_{\tilde{U}}=\left.\tilde{\omega}\right|_{\tilde{U}}$, então $\omega$ é um elemento de $\mathcal{P}$.

Definição 1.6.2 Sejam $\mathcal{P}_{1}$ um pseudogrupo de difeomorfirsmos (isometrias) de uma variedade $M$ e $\mathcal{P}_{2}$ um pseudogrupo de difeomorfismos (isometrias) de uma variedade $N$. Uma equivalência entre $\mathcal{P}_{1}$ e $\mathcal{P}_{2}$ é uma família maximal $\Phi$ de difeomorfismos (isometrias) entre abertos de $M$ e $N$ tal que

1. Os dominios dos elementos de $\Phi$ é uma cobertura por abertos de $M$ e as imagens dos elementos de $\Phi$ é uma cobertura por abertos de $N$;

2. se $\omega_{1} \in \mathcal{P}_{1}, \omega_{2} \in \mathcal{P}_{2}$ e $\phi \in \Phi$, então $\omega_{2} \circ \phi \circ \omega_{1}$ é um elemento de $\Phi$;

3. $\omega \in \mathcal{P}_{1}, \tilde{\omega} \in \mathcal{P}_{2}$ e $\phi_{1}, \phi_{2} \in \Phi$, então $\phi_{2} \circ \omega \circ \phi_{1}^{-1} \in \mathcal{P}_{2}$ e $\phi_{1}^{-1} \circ \tilde{\omega} \circ \phi_{2} \in \mathcal{P}_{1}$.

Observação 1.6.1 Seja $\left\{\left(M_{i}, \bar{\phi}_{i}, \Gamma_{i}\right)\right\}_{i \in I}$ um atlas para um orbifold X. Definimos $M:=\bigcup_{i \in I} M_{i}$, i.e, $M$ é a união disjunta da familia $\left\{M_{i}\right\}_{i \in I}$, e neste caso, cada $M_{i}$ é uma componente conexa de $M$. Definimos $\bar{\phi}$ como a união da familia $\left\{\bar{\phi}_{i}\right\}_{i \in I}$. Agora, cada difeomorfismo local $h_{i j}$ de mudança de cartas do atlas $\left\{\left(M_{i}, \bar{\phi}_{i}, \Gamma_{i}\right)\right\}_{i \in I}$ é um difeomorfismo local de $M$ em M. Assim, a familia $\left\{h_{i j}\right\}$ de mudança de cartas do atlas $\left\{\left(M_{i}, \bar{\phi}_{i}, \Gamma_{i}\right)\right\}_{i \in I}$ define um pseudogrupo $\mathcal{P}$, e neste caso $\mathcal{P}$ é chamado o pseudogrupo de mudança de cartas de $X$ com respeito ao atlas $\left\{\left(M_{i}, \bar{\phi}_{i}, \Gamma_{i}\right)\right\}_{i \in I}$.

Dizemos que dois pontos $x, y$ estão na mesma órbita de $\mathcal{P}$ se existe $h \in \mathcal{P}$ tal que $h(x)=y$. Essa relação define uma relação de equivalência em $M$ e as classes são chamadas órbitas de $\mathcal{P}$. Agora, o espaço de órbitas com topologia quociente é denotado como $M / \mathcal{P}$, e a aplicação $\bar{\phi}$ induz um homeomorfismo entre $M / \mathcal{P}$ e $X$.

Seja $\left\{\left(N_{j}, \bar{\psi}_{j}, \tilde{\Gamma}_{j}\right)\right\}_{j \in J}$ outro atlas que define a mesma estrutura diferenciável em $X$. Definamos por $\tilde{\mathcal{P}}$ é pseudogrupo que define o atlas $\left\{\left(N_{j}, \bar{\psi}_{j}, \tilde{\Gamma}_{j}\right)\right\}_{j \in J}$. Dizemos que $\mathcal{P}$ e $\tilde{\mathcal{P}}$ são equivalentes se existe um pseudogrupo $\mathcal{R}$ de difeomorfismos locais de $M \cup N$, onde a união é disjunta e $N:=\bigcup_{j \in J} N_{j}$, tal que $\left.\mathcal{R}\right|_{M}=\mathcal{P}$ e $\left.\mathcal{R}\right|_{N}=\tilde{\mathcal{P}}$ e tal que as inclusões $i_{M}: M \rightarrow M \cup N$ e $i_{N}: N \rightarrow M \cup N$ induzem homeomorfismos $M / \mathcal{P} \rightarrow[M \cup N] / \mathcal{R}$ e $N / \tilde{\mathcal{P}} \rightarrow[M \cup N] / \mathcal{R}$, respectivamente. 


\subsection{Definição Alternativa de Orbifold}

Definição 1.7.1 Um orbifold diferenciável (Riemanniano) $X$ é uma classes de equivalência de pseudogrupos $\mathcal{P}$ de difeomorfismos (isometrias) locais de uma variedade diferenciável (Riemanniana) $M$, tal que

1. O espaço de órbitas $M / \mathcal{P}$ é Hausdorff;

2. para cada $x \in M$ existe uma vizinhança aberta $x \in U \subset M$ tal que $\left.\mathcal{P}\right|_{U}$ é gerado por um grupo finito de difeomorfismos (isometrias) de $U$.

Neste caso o espaço $M / \mathcal{P}$ é o espaço subjacente do orbifold $X$.

Se $\Gamma$ é um grupo discreto de Dif $(M)(I s o(M))$ que age propriamente sobre uma variedade (Riemanniana) conexa $M$, então $M / \Gamma$ tem estrutura de good orbifold, e neste caso o pseudogrupo de mudança de cartas é gerado pelos elementos de $\Gamma$.

Exemplo 1.7.1 Um exemplo importante de um pseudogrupo de isometrias locais é o pseudogrupo de holonomia de uma folheação Riemanniana: seja $\mathfrak{F}$ uma folheação Riemanniana de codimensão $k$ de uma variedade Riemanniana $\left(M^{n}, g\right)$, então $\mathfrak{F}$ pode ser descrita por uma cobertura aberta $\left\{U_{i}\right\}$ de $M$ com submersões $f_{i}:\left(U_{i}, g\right) \rightarrow\left(V_{i}, h\right)$, onde $\left(V_{i}, h\right)$ é uma subvariedade Riemanniana de dimensão $k$, tal que existem isometrias

$$
\omega_{i j}: f_{i}\left(U_{i} \cap U_{j}\right) \rightarrow f_{j}\left(U_{i} \cap U_{j}\right), \operatorname{com} f_{j}=\omega_{i j} \circ f_{i} .
$$

Os elementos $\omega_{i j}$ que agem sobre $N:=\bigcup_{i \in I} V_{i}$ geram um pseudogrupo $\mathcal{P}$ de isometrias que é chamado o psudogrupo de holonomia de $\mathfrak{F}$.

Exemplo 1.7.2 Um exemplo importante de um orbifold Riemanniano é o espaço de folhas $M / \mathfrak{F}$ de uma folheaçẫo Riemanniana $\mathfrak{F}$ com folhas mergulhadas fechadas de uma variedade Riemaniana $(M, g)$. Neste caso $M / \mathfrak{F}$ é isométrico ao espaço $N / \mathcal{P}$, onde $N$ e $\mathcal{P}$ são definidos no exemplo 1.7.1. A seguinte proposição pode ser vista como a recíproca do exemplo 1.7.2 (vide [AB15]).

Proposição 1.7.1 Cada orbifold Riemanniano $N / \mathcal{P}$ é o espaço de folhas de uma folheação Riemanniana com folhas mergulhadas fechadas. 


\subsection{Orbifolds Métricos}

Proposição 1.8.1 Seja $\mathbb{V}$ um espaço Euclidiano. Sejam $\Gamma$ e $\tilde{\Gamma}$ dois subgrupos finitos de $\mathbb{O}(\mathbb{V})$, e suponhamos que existe um elemento $\phi$ em $\mathbb{O}(\mathbb{V})$ que satisfaz $\tilde{\Gamma}=\phi \Gamma \phi^{-1}$. Então os espaços $\mathbb{V} / \Gamma e$ $\mathbb{V} / \tilde{\Gamma}$ são isométricos.

Demonstração. Dados $\omega \in \Gamma$ e $x \in \mathbb{V}$, quaisquer, temos que

$$
\phi(\omega(x))=\phi \circ \omega \circ \phi^{-1}(\phi(x)) .
$$

Então, dado que $\tilde{\Gamma}=\phi \circ \Gamma \circ \phi^{-1}$, temos que $\phi(\omega(x))$ e $\phi(x)$ estão na mesma $\tilde{\Gamma}$ - órbita. Assim, a seguinte aplicação

$$
\begin{aligned}
\bar{\phi}: \mathbb{V} / \Gamma & \longrightarrow \mathbb{V} / \tilde{\Gamma} \\
\Gamma x & \longmapsto \tilde{\Gamma} \phi(x)
\end{aligned}
$$

está bem definida. Por ser bem definida, $\bar{\phi}$ é injetiva. E é fácil ver que ela é sobrejetora, e portanto $\bar{\phi}$ é uma bijeção. Sejam $\pi: \mathbb{V} \rightarrow \mathbb{V} / \Gamma$ e $\eta: \mathbb{V} \rightarrow \mathbb{V} / \tilde{\Gamma}$ as aplicações quocientes associadas aos grupos $\Gamma$ e $\tilde{\Gamma}$, respectivamente. Temos que a aplicação $\eta \circ \phi$ é $\Gamma$-invariante. Logo, dado que $\eta \circ \phi$ é aberta e contínua, temos que $\bar{\phi}$ é aberta e contínua, respectivamente. Portanto $\bar{\phi}$ é um homeomorfismo. Vamos provar que $\bar{\phi}$ é uma isometria. Sejam $x_{1}$ e $x_{2}$ dois elementos quaisquer de $\mathbb{V}$, então

$$
\begin{aligned}
d_{\tilde{\Gamma}}\left(\bar{\phi}\left(\Gamma x_{1}\right), \bar{\phi}\left(\Gamma x_{2}\right)\right) & =d_{\tilde{\Gamma}}\left(\tilde{\Gamma} \phi\left(x_{1}\right), \tilde{\Gamma} \phi\left(x_{2}\right)\right) & & \text { pela definição de } \bar{\phi} \\
& =\inf \left\{d\left(\phi\left(x_{1}\right), \tilde{\omega}\left(\phi\left(x_{2}\right)\right)\right): \tilde{\omega} \in \tilde{\Gamma}\right\} & & \text { por definição de } d_{\tilde{\Gamma}} \\
& =\inf \left\{d\left(\phi\left(x_{1}\right), \phi\left(\omega\left(x_{2}\right)\right)\right): \omega \in \Gamma\right\} & & \text { dado que } \tilde{\Gamma}=\phi \Gamma \phi^{-1} \\
& =\inf \left\{d\left(x_{1}, \omega\left(x_{2}\right)\right): \omega \in \Gamma\right\} & & \phi \text { é uma isometria } \\
& =d_{\Gamma}\left(\Gamma x_{1}, \Gamma x_{2}\right) & & \text { pela definição de } d_{\Gamma} .
\end{aligned}
$$

Então $\bar{\phi}$ é uma isometria.

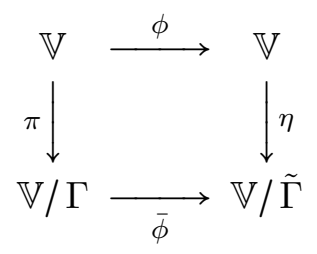

Lema 1.8.1 Sejam $M$ uma variedade Riemanniana e $\Gamma$ um subgrupo finito de isometrias de $M$. Sejam $\gamma:[-a, a] \rightarrow M$ uma geodésica minimizante e $\pi: M \rightarrow M / \Gamma$ a aplicação quociente da ação 
de $\Gamma$ sobre $M$. Vamos supor que $\Gamma$ fixa $\gamma(0)$, então $\pi \circ \gamma:[0, a] \rightarrow M / \Gamma$ é uma geodésica métrica em $M / \Gamma$. Além disso, $\pi \circ \gamma:[-a, a] \rightarrow M / \Gamma$ é uma geodésica métrica se e somente se $\Gamma_{\gamma(t)}=\Gamma$, para todo $t \in[-a, a]$.

Demonstração. Primeiro observemos que em $M / \Gamma$ podemos induzir a métrica dada pela ação de $\Gamma$ sobre $M$, pois $\Gamma$ é um grupo finito.

Vamos provar a primeira afirmação. Seja $t \in[0, a]$, então

$$
\begin{aligned}
d_{\Gamma}(\Gamma \gamma(0), \Gamma \gamma(t)) & =\inf \{d(\omega \gamma(0), \gamma(t)): \omega \in \Gamma\} & & \text { pela difinição de } d_{\Gamma} \\
& =\inf \{d(\gamma(0), \gamma(t)): \omega \in \Gamma\} & & \text { dado que } \Gamma_{\gamma(0)}=\Gamma \\
& =d(\gamma(0), \gamma(t))=t & & \text { pois } \gamma \text { é minimizante. }
\end{aligned}
$$

Logo

$$
d_{\Gamma}(\Gamma \gamma(0), \Gamma \gamma(t))=t, \quad \text { para todo } t \in[0, a]
$$

Sejam $0<t_{1}<t_{2} \leq a$. Pela definição de $d_{\Gamma}$, vamos ter que

$$
d_{\Gamma}\left(\Gamma \gamma\left(t_{1}\right), \Gamma \gamma\left(t_{2}\right)\right) \leq d\left(\gamma\left(t_{1}\right), \gamma\left(t_{2}\right)\right)=t_{2}-t_{1}
$$

Agora

$$
\begin{aligned}
t_{2} & =d_{\Gamma}\left(\Gamma \gamma(0), \Gamma \gamma\left(t_{2}\right)\right) & & \text { pela equação (1.5) } \\
& \leq d_{\Gamma}\left(\Gamma \gamma(0), \Gamma \gamma\left(t_{1}\right)\right)+d_{\Gamma}\left(\Gamma \gamma\left(t_{1}\right), \Gamma \gamma\left(t_{2}\right)\right) & & \text { por desigualdade triangular } \\
& =t_{1}+d_{\Gamma}\left(\Gamma \gamma\left(t_{1}\right), \Gamma \gamma\left(t_{2}\right)\right) & & \text { pela equação (1.5). }
\end{aligned}
$$

Portanto, pelas equações (1.6) e (1.7), temos que

$$
t_{2}-t_{1} \leq d_{\Gamma}\left(\Gamma \gamma\left(t_{1}\right), \Gamma \gamma\left(t_{2}\right)\right) \leq t_{2}-t_{1}
$$

Logo temos a prova da primeira afirmação. Observe que, da mesma maneira, podemos concluir que $\pi \circ \gamma(-t):[0, a] \rightarrow M / \Gamma$ é uma geodésica métrica em $M / \Gamma$.

Vamos provar a segunda afirmação. Primeiro suponhamos que $\pi \circ \gamma$ é uma geodésica métrica em $M / \Gamma$, mas vamos supor que não temos a conclusão, i.e., vamos supor que existe um $t_{0} \in[-a, a]$ tal que $\Gamma_{\gamma\left(t_{0}\right)} \neq \Gamma$. Para continuar nossa prova basta supor que a imagem de $\gamma$ está contida numa 
vizinhaça normal de $\gamma(0)$. Assim, seja $v \in T_{\gamma(0)} M$ tal que $|v|=a$ e $\gamma(t)=\exp _{\gamma(0)}(t v)$, para $-1 \leq t \leq 1$. Dado $\omega \in \Gamma$, temos que

$$
\omega \gamma(t)=\omega \exp _{\gamma(0)}(t v)=\exp _{\gamma(0)}\left((d \omega)_{\gamma(0)} t v\right)=\exp _{\gamma(0)}\left(t(d \omega)_{\gamma(0)} v\right)
$$

Observe que da equação (1.8) podemos concluir que $\omega \exp _{\gamma(0)}(s v) \neq \exp _{\gamma(0)}(v)$ se e somente se $\omega \exp _{\gamma(0)}(t v) \neq \exp _{\gamma(0)}(t v)$, para todo $t \in[-1,1] \backslash\{0\}$. Portanto, o grupo de isotropia ao longo da geodésica $\gamma(t)$ permanece constante salvo quando $t=0$. Em particular, se existe algum $\bar{t} \epsilon$ $[-a, a] \backslash\{0\}$ tal que $\Gamma_{\gamma(\bar{t})}=\{e\}$, onde $e$ é o elemento identidade de $\Gamma$, então $\Gamma_{\gamma(t)}=\{e\}$, para todo $t \in[-a, a] \backslash\{0\}$. Dado que estamos supondo que $t_{0}$ é tal que $t_{0} \neq 0$ e $\Gamma_{\gamma\left(t_{0}\right)} \neq \Gamma$, então, pelo provado antes, temos que $\Gamma \neq \Gamma_{\gamma(t)}=\Gamma_{\gamma\left(t_{0}\right)}$, para todo $t \in[-a, a] \backslash\{0\}$. Logo, em particular, para todo $t \in(0, a]$ existe $\omega \in \Gamma$ tal que $\omega \gamma(t) \neq \gamma(t)$. Dado que por hipótese temos que $\pi \circ \gamma$ é uma geodésica métrica em $M / \Gamma$, temos que $d_{\Gamma}(\Gamma \gamma(-t), \Gamma \gamma(t))=2 t$, para todo $t \in(0, a]$.

Agora

$$
\begin{array}{rlrl}
d_{\Gamma}(\Gamma \gamma(-t), \Gamma \gamma(t)) & \leq d(\gamma(-t), \omega \gamma(t)) & & \text { por difinição de } d_{\Gamma} \\
& <d(\gamma(-t), \gamma(0))+d(\gamma(0), \omega \gamma(t)) & & \text { desigualdade triangular } \\
& =d(\gamma(-t), \gamma(0))+d\left(\omega^{-1} \gamma(0), \gamma(t)\right) & \\
& =d(\gamma(-t), \gamma(0))+d(\gamma(0), \gamma(t)) & & \text { pois } \Gamma=\Gamma_{\gamma(0)} \\
& =2 t & & \text { dado que } \gamma \text { é minimizante. }
\end{array}
$$

Observemos que a seguinte desigualdade triangular

$$
d(\gamma(-t), \omega \gamma(t))<d(\gamma(-t), \gamma(0))+d(\gamma(0), \omega \gamma(t))
$$

é estrita, pois $\omega \gamma(t)$ não está na imagem de $\gamma$. Logo, como mostrados acima, temos que

$$
2 t=d_{\Gamma}(\Gamma \gamma(-t), \Gamma \gamma(t))<2 t
$$

que é um absurdo. Portanto, se $\pi \circ \gamma:[-a, a] \rightarrow M / \Gamma$ é uma geodésica métrica, então $\Gamma_{\gamma(t)}=\Gamma$, para todo $t \in[-a, a]$.

Agora vamos provar a outra direção da segunda afirmação. Suponhamos que $\Gamma_{\gamma(t)}=\Gamma$, para todo $t \in[-a, a]$. Em particular temos que $\Gamma$ fixa $\gamma(-a)$. Então, pela primeira parte da proposição, temos que $\pi \circ \gamma:[-a, a] \rightarrow M / \Gamma$ é uma geodésica métrica em $M / \Gamma$. 
Logo temos a prova da segunda parte da proposição.

Observação 1.8.1 A prova do Lema 1.8.1 também pode ser obtida pelo seguinte resultado (vide [AB15], Lema 3.70):

Sejam $\mu: \Gamma \times M \rightarrow M$ uma ação por isometrias e seja $\beta:[0,1] \rightarrow M$ uma geodésica que atende a distância entre as orbitas $\Gamma(\beta(0))$ e $\Gamma(\beta(1))$. Então existe um subgrupo $\Gamma_{0} \subset \Gamma$ tal que $\Gamma_{\beta(t)}=\Gamma_{0}$, para todo $t \in(0,1)$ e $\Gamma_{0}$ é um subgrupo de $\Gamma_{\beta(0)}$ e de $\Gamma_{\beta(1)}$.

Lema 1.8.2 Sejam $M$ e $N$ duas variedades Riemannianas, $\Gamma$ e $\tilde{\Gamma}$ dois subgrupos discretos de isometrias de $M$ e $N$, respectivamente, que agem propriamente. Sejam $\pi: M \rightarrow M / \Gamma$ e $\eta: N \rightarrow N / \tilde{\Gamma}$ as aplicações quocientes associadas aos grupos $\Gamma$ e $\tilde{\Gamma}$, respectivamente, e vamos supor que

$$
\bar{\psi}: M / \Gamma \longrightarrow N / \tilde{\Gamma}
$$

é uma isometria de espaços métricos. Sejam x em $M$ e $y \in N$ tais que $\bar{\psi}(\pi(x))=\eta(y)$, então $\Gamma_{x}$ é trivial se e somente se $\tilde{\Gamma}_{y}$ é trivial.

Demonstração. Vamos supor que $\Gamma_{x}$ é trivial, mas que $\tilde{\Gamma}_{y}$ não é trivial. Pelo Teorema 1.2.5, existe $\tilde{r}>0$ tal que para todo $r \leq \tilde{r},\left.\pi\right|_{B_{r}(x)}: B_{r}(x) \rightarrow B_{r}(x) / \Gamma$ é uma isometria. Da mesma maneira, para $y$ existem $\tilde{s}>0$ e uma isometria $\rho: B_{s}(y) / \tilde{\Gamma}_{y} \rightarrow B_{s}(y) / \tilde{\Gamma}$, para todo $s \leq \tilde{s}$. Além disso, se definimos $\eta_{y}: B_{s}(y) \rightarrow B_{s}(y) / \tilde{\Gamma}_{y}$ como a aplicação quociente associada à ação de $\tilde{\Gamma}_{y}$ sobre $B_{s}(y)$, então $\left.\eta\right|_{B_{s}(y)}=\rho \circ \eta_{y}$. Podemos supor, sem perda de generalidade, que as bolas $B_{\tilde{r}}(x)$ e $B_{\tilde{s}}(y)$ são bolas normais de $x$ e $y$, respectivamente. Seja $\bar{r}=\min \{\tilde{r}, \tilde{s}\}$, então para todo $r \leq \bar{r}$, temos que $\left.\rho^{-1} \circ \bar{\psi} \circ \pi\right|_{B_{r}(x)}: B_{r}(x) \rightarrow B_{r}(y) / \tilde{\Gamma}_{y}$ é uma isometria de espaços métricos. Definamos

$$
\Omega_{x}:=\left\{\gamma:(-\bar{r}, \bar{r}) \rightarrow B_{\bar{r}}(x) \mid \gamma \text { é uma geodésica e } \gamma(0)=x\right\}
$$

e seja $\gamma$ em $\Omega_{x}$. Dado que $\gamma$ é uma geodésica minimizante, temos que

$$
\rho^{-1} \circ \bar{\psi} \circ \pi \circ \gamma:(-\bar{r}, \bar{r}) \rightarrow B_{\bar{r}}(y) / \tilde{\Gamma}_{y}
$$

é uma geodésica métrica e $\eta_{y}(y)=\rho^{-1} \circ \bar{\psi} \circ \pi \circ \gamma(0)$. Definamos

$$
\Lambda:=\left\{\bar{\beta}:(-\bar{r}, \bar{r}) \rightarrow B_{\bar{r}}(y) / \tilde{\Gamma}_{y}: \bar{\beta}=\rho^{-1} \circ \bar{\psi} \circ \pi \circ \gamma, \text { para alguma } \gamma \in \Omega_{x}\right\} .
$$


Agora, temos que

$$
B_{\bar{r}}(y) / \tilde{\Gamma}_{y}=\bigsqcup_{\bar{\beta} \in \Lambda, t \in(-\bar{r}, \bar{r})} \bar{\beta}(t)
$$

sendo esta uma união disjunta dois a dois. E também temos que, por [Rat06] (Teorema 13.1.6), para cada $\bar{\beta} \in \Lambda$ existe uma geodésica $\tilde{\beta}:(-\bar{r}, \bar{r}) \rightarrow B_{\bar{r}}(y)$ tal que $\tilde{\beta}(0)=y$ e $\eta_{y}(\tilde{\beta}(t))=\bar{\beta}(t)$, para todo $t \in(-\bar{r}, \bar{r})$. Dado que $\bar{\beta}$ é uma geodésica métrica em $B_{\bar{r}}(y) / \tilde{\Gamma}_{y}$ temos, pelo Lema 1.8.1, que $\tilde{\Gamma}_{\tilde{\beta}(t)}=\tilde{\Gamma}$, para todo $t \in(-\bar{r}, \bar{r})$. Logo cada ponto em $B_{\bar{r}}(y)$ tem como grupo de isotropia o grupo $\tilde{\Gamma}$. Mas isso é um absurdo pois, por [Rat06] (Teorema 13.2.4), o conjunto dos pontos regulares em $B_{\bar{r}}(y)$ é um conjunto aberto e denso de $B_{\bar{r}}(y)$. Logo $\tilde{\Gamma}_{y}$ é trivial.

Proposição 1.8.2 Sejam $\mathbb{V}$ um espaço Euclidiano, $\Gamma$ e $\tilde{\Gamma}$ dois subgrupos finitos de $\mathbb{O}(\mathbb{V})$, e supomos que os espaços $\mathbb{V} / \Gamma$ e $\mathbb{V} / \tilde{\Gamma}$ são isométricos. Então os grupos $\Gamma$ e $\tilde{\Gamma}$ são conjugados em $\mathbb{O}(\mathbb{V})$.

Demonstração. Sejam $\pi: \mathbb{V} \rightarrow \mathbb{V} / \Gamma$ e $\eta: \mathbb{V} \rightarrow \mathbb{V} / \tilde{\Gamma}$ as aplicações quocientes associadas aos grupos $\Gamma$ e $\tilde{\Gamma}$, respectivamente. E seja $\bar{\psi}: \mathbb{V} / \Gamma \rightarrow \mathbb{V} / \tilde{\Gamma}$ a isometria que temos por hipótese.

Nosso primeiro objetivo é provar que existe uma isometria $\psi: \mathbb{V} \rightarrow \mathbb{V}$, tal que $\eta \circ \psi=\bar{\psi} \circ \pi$. Para provar este fato, vamos fazer indução sobre a dimensão do espaço Euclidiano $\mathbb{V}$.

Como caso inicial, vamos supor que $\operatorname{dim}(\mathbb{V})$ é 1 . Neste caso, podemos supor que $\mathbb{V} \simeq \mathbb{R}$. Assim, temos os seguinte casos:

1. $\Gamma=\{e\}$ e $\tilde{\Gamma}=\{e\}$. Assim, $\mathbb{R} / \Gamma=\mathbb{R} / \tilde{\Gamma}=\mathbb{R}$

2. $\Gamma=\{e,-e\}=\tilde{\Gamma}$. Então $\mathbb{R} / \Gamma=[0, \infty)=\mathbb{R} / \tilde{\Gamma}$

3. $\Gamma=\{e\}$ e $\tilde{\Gamma}=\{e,-e\}$. Logo $\mathbb{R} / \Gamma=\mathbb{R}$ e $\mathbb{R} / \tilde{\Gamma}=[0, \infty)$.

Observemos que no último caso temos que na ação de $\tilde{\Gamma}$ sobre $\mathbb{R}$, 0 é o único ponto de $\mathbb{R}$ que não possui grupo de isotropia trivial, pois seu grupo de isotropia é o grupo $\tilde{\Gamma}$, mas a ação de $\Gamma$ sobre $\mathbb{R}$ é livre. Então, pelo Lema 1.8.2, neste caso $\mathbb{R} / \Gamma=\mathbb{R}$ e $\mathbb{R} / \tilde{\Gamma}=[0, \infty)$ não podem ser isométricos. Agora, os dois primeiros casos são os únicos onde é satisfeita a hipótese da proposição, e podemos escolher $\psi$ como a isometria identidade de $\mathbb{V}$, obtendo que $\eta \circ \psi=\bar{\psi} \circ \pi$. Mais ainda, não precisamos da existência de $\psi$ para obter que os grupos $\Gamma$ e $\tilde{\Gamma}$ são conjugados em $\mathbb{O}(\mathbb{V})$, pois $\Gamma=\tilde{\Gamma}$. Assim temos a prova para $\operatorname{dim}(\mathbb{V})=1$.

Para $\operatorname{dim}(\mathbb{V}):=n>1$ vamos trabalhar em $\mathbb{S}^{n-1}:=\left\{x \in \mathbb{V}:\|x\|_{\mathbb{V}}=1\right\}$. Pois, por [Rat06] (Teorema 2.1.3), temos que cada transformação ortogonal de $\mathbb{R}^{n}$ se restringe a uma isometria de $\mathbb{S}^{n-1}$, e cada 
isometria de $\mathbb{S}^{n-1}$ se estende a uma única transformação ortogonal de $\mathbb{R}^{n}$.

Supomos que $\operatorname{dim}(\mathbb{V})=2$. Dado que, por hipótese, os espaços $\mathbb{V} / \Gamma$ e $\mathbb{V} / \tilde{\Gamma}$ são isométricos, temos que $\mathbb{S}^{1} / \Gamma$ e $\mathbb{S}^{1} / \tilde{\Gamma}$ são isométricos. Também, por hipótese, temos que $\Gamma$ e $\tilde{\Gamma}$ são subgrupos finitos de $\mathbb{O}(\mathbb{V})$, logo, por [Kan01] (Teorema 1), $\Gamma$ e $\tilde{\Gamma}$ somente podem pertencer a uma das duas seguintes classes de grupos: um grupo de rotações ou num grupo diedral gerado por reflexões. Observemos que se $\Gamma$ e $\tilde{\Gamma}$ estão em classes diferentes então $\mathbb{S}^{1} / \Gamma$ e $\mathbb{S}^{1} / \tilde{\Gamma}$ não podem ser isométricos. Com efeito, se eles estão em classes diferentes, supomos que $\Gamma$ é um grupo de rotações e que $\tilde{\Gamma}$ é um grupo diedral gerado por reflexões, então $\Gamma$ agiria livremente sobre $\mathbb{S}^{2}$ e $\tilde{\Gamma}$ não, assim, pela Proposição 1.8.2, os espaços $\mathbb{S}^{1} / \Gamma$ e $\mathbb{S}^{1} / \tilde{\Gamma}$ não podem ser isométricos. Portanto não podemos ter a hipótese de nossa proposição. Logo temos somente os dois seguintes casos.

1. $\Gamma$ e $\tilde{\Gamma}$ são grupos de rotações. Assim, $\Gamma \simeq \mathbb{Z} / k \mathbb{Z}$ e $\tilde{\Gamma} \simeq \mathbb{Z} / m \mathbb{Z}$. Dado que $\mathbb{S}^{1} / \Gamma$ e $\mathbb{S}^{1} / \tilde{\Gamma}$ são isométricos, temos que $m=k$. Neste caso podemos escolher $\psi$ como a isometria identidade de $\mathbb{S}^{2}$, e assim temos a prova neste caso.

2. $\Gamma$ e $\tilde{\Gamma}$ são grupos de diedrais gerados por reflexões. Então $\Gamma \simeq \mathbb{Z} / k \mathbb{Z} \rtimes \mathbb{Z} / 2 \mathbb{Z}$ e $\tilde{\Gamma} \simeq \mathbb{Z} / m \mathbb{Z} \rtimes$ $\mathbb{Z} / 2 \mathbb{Z}$, onde $k$ e $m$ estão no conjunto $\{0,1,2, \ldots\}$. Suponhamos que $\Gamma$ é gerado por as reflexões $r_{1}$ e $r_{2}$ e $\tilde{\Gamma}$ é gerado pelas reflexões $\tilde{r}_{1}$ e $\tilde{r}_{2}$, i.e.,

$$
\Gamma=\left\langle r_{1}, r_{2}>=<r_{1}, r_{1} r_{2}>\right.
$$

e

$$
\tilde{\Gamma}=<\tilde{r}_{1}, \tilde{r}_{2}>=<\tilde{r}_{1}, \tilde{r}_{1} \tilde{r}_{2}>
$$

onde $r_{1} r_{2}$ e $\tilde{r}_{1} \tilde{r}_{2}$ são duas rotações com um ângulo $\frac{\pi}{k}$ e $\frac{\pi}{m}$, respectivamente. Agora, dado que os diâmetros de $\mathbb{S}^{1} / \Gamma$ e $\mathbb{S}^{1} / \tilde{\Gamma}$ são $\frac{2 \pi}{k}$ e $\frac{2 \pi}{m}$, respectivamente, e $\mathbb{S}^{1} / \Gamma$ e $\mathbb{S}^{1} / \tilde{\Gamma}$ são isométricos, então $k$ e $m$ são iguais.

Sejam $H_{1}, H_{2}, \tilde{H}_{1}$ e $\tilde{H}_{2}$ os subespaços fixados por $r_{1}, r_{2}, \tilde{r}_{1}$ e $\tilde{r}_{2}$, respectivamente. Sejam $x_{1} \in H_{1} \cap \mathbb{S}^{1}$ e $x_{2} \in H_{2} \cap \mathbb{S}^{1}$ tais que o ângulo entre os vetores $\vec{x}_{1}$ e $\vec{x}_{2}$ é $\frac{\pi}{m}$, e $y_{1} \in \tilde{H}_{1} \cap \mathbb{S}^{1}$ e $y_{2} \in \tilde{H}_{2} \cap \mathbb{S}^{1}$ tais que o ângulo entre os vetores $\vec{y}_{1}$ e $\vec{y}_{2}$ é $\frac{\pi}{m}$. Agora, dado que $\mathbb{O}_{2}(\mathbb{R})$ age transitivamente sobre $\mathbb{S}^{1}$, definamos $\psi \in \mathbb{O}_{2}(\mathbb{R})$ tal que $\psi\left(x_{1}\right)=y_{1}$. É fácil ver que $\psi\left(x_{2}\right)=y_{2}$. Observemos que $\tilde{r}_{1}$ é determinado por $\pm y_{1}$ e $\tilde{r}_{2}$ é determinado por $\pm y_{2}$. Agora, temos que $\psi \circ r_{1} \circ \psi^{-1}=\tilde{r}_{1}$ e $\psi \circ r_{2} \circ \psi^{-1}=\tilde{r}_{2}$, pois $\psi \circ r_{1} \circ \psi^{-1}\left( \pm y_{i}\right)= \pm y_{i}$, para $i=1,2$, respectivamente. Portanto $\psi \Gamma \psi^{-1}=\tilde{\Gamma}$. 

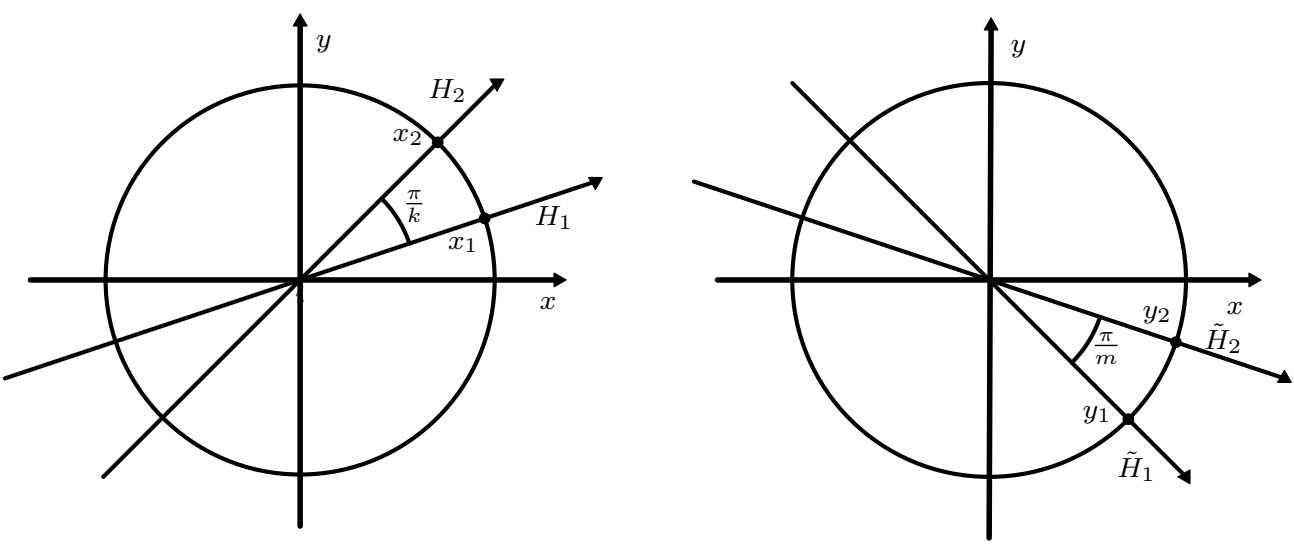

Figura 1.3: Caso $n=2, \operatorname{com} \Gamma$ e $\tilde{\Gamma}$ gerados por reflexões.

Nossa hipótese de indução consiste em supor que o resultado é válido para $\operatorname{dim}(\mathbb{V})=n$, i.e., supor que existe uma isometria $\psi: \mathbb{S}^{n-1} \rightarrow \mathbb{S}^{n-1}$, tal que $\eta \circ \psi=\bar{\psi} \circ \pi$.

Vamos provar para $\operatorname{dim}(\mathbb{V})=n+1$. Definamos $X:=\mathbb{S}^{n} / \Gamma$ e $Y:=\mathbb{S}^{n} / \tilde{\Gamma}$, e, por simplicidade, sejam $\pi: \mathbb{S}^{n} \rightarrow \mathbb{S}^{n} / \Gamma$ e $\eta: \mathbb{S}^{n} \rightarrow \mathbb{S}^{n} / \tilde{\Gamma}$ as aplicações quocientes associadas aos grupos $\Gamma$ e $\tilde{\Gamma}$, respectivamente. Também, por simplicidade, seja $\bar{\psi}:=\left.\bar{\psi}\right|_{\mathbb{S}^{n} / \Gamma}: \mathbb{S}^{n} / \Gamma \rightarrow \mathbb{S}^{n} / \tilde{\Gamma}$, a isometria que temos por hipótese. Observemos que se $\Gamma$ age livremente sobre $\mathbb{S}^{n}$, então, pela Proposição 1.8.2, temos que $\tilde{\Gamma}$ também age da mesma maneira sobre $\mathbb{S}^{n}$. Neste caso $X$ e $Y$ são espaços-forma e $\mathbb{S}^{n}$ é simplesmente conexo, pois $n \geq 2$, logo, por [Rat06] (Teorema 8.1.5), $\Gamma$ e $\tilde{\Gamma}$ são conjugados em $\mathbb{O}(\mathbb{V})$. E assim temos a prova neste caso.

Agora vamos provar o caso no qual $\Gamma$ e $\tilde{\Gamma}$ não agem livremente sobre $\mathbb{S}^{n}$. Sejam $\bar{x} \in X$ e $\bar{y} \in Y$, tais que $\bar{\psi}(\bar{x})=\bar{y}$. Suponhamos que $\bar{x}$ é tal que para um $x \in \pi^{-1}(\bar{x}), x$ é um ponto regular, i.e., $\Gamma_{x}=\{e\}$, onde $e$ é o elemento identidade de $\Gamma$. E sabemos que isso acontece para todo elemento de $\pi^{-1}(\bar{x})$. Fixemos $x \in \pi^{-1}(\bar{x})$ e $y \in \eta^{-1}(\bar{y})$. Então pela Proposição 1.8.2 temos que $y$ é um ponto regular na ação de $\tilde{\Gamma}$ sobre $\mathbb{S}^{n}$, i.e., $\tilde{\Gamma}_{y}=\{\tilde{e}\}$, onde $\tilde{e}$ é o elemento identidade de $\tilde{\Gamma}$. Agora, pelo Teorema 1.2.5, existe $\tilde{r}>0$ tal que se $r \leq \tilde{r}$, então $\left.\pi\right|_{B_{r}(x)}: B_{r}(x) \rightarrow B_{r}(x) / \Gamma$ é uma isometria. Da mesma maneira, para $y$ existe $\tilde{s}>0$ tal que se $s \leq \tilde{s}$, então $\left.\eta\right|_{B_{s}(y)}: B_{s}(y) \rightarrow B_{s}(y) / \tilde{\Gamma}$ é uma isometria. Seja $r_{0}:=$ $\min \{\tilde{r}, \tilde{s}\}$, então para todo $r \leq r_{0}$ temos que

$$
\left.\eta^{-1} \circ \bar{\psi} \circ \pi\right|_{B_{r}(x)}: B_{r}(x) \rightarrow B_{r}(y)
$$

é uma isometria e $\eta^{-1} \circ \bar{\psi} \circ \pi(x)=y$. Agora, por [Wol11] (Teorema 2.3.12), existe uma única isometria

$$
\psi: \mathbb{S}^{n} \rightarrow \mathbb{S}^{n}
$$


tal que $\psi(x)=y$ e

$$
\left.\psi\right|_{B_{r(x)}}=\left.\eta^{-1} \circ \bar{\psi} \circ \pi\right|_{B_{r}(x)} .
$$

Agora, nosso objetivo é provar que o seguinte diagrama é comutativo.

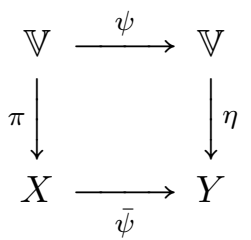

Para provar isso basta com demostrar que se $\alpha: \mathbb{R} \rightarrow \mathbb{S}^{n}$ é uma geodésica parametrizada pelo comprimento de arco e tal que $\alpha(0)=x$, então $\eta \circ \psi(\alpha(t))=\bar{\psi} \circ \pi(\alpha(t))$, para todo $0 \leq t \leq 2 \pi$. Seja $\gamma:[0, a] \rightarrow \mathbb{S}^{n}$ uma geodésica parametrizada pelo comprimento de arco, tal que $\gamma(0)=x$ e $\gamma([0, a]) \subset B_{r}(x)$, então temos que

$$
\eta \circ \psi(\gamma(t))=\bar{\psi} \circ \pi(\gamma(t)), \text { para todo } t \in[0, a]
$$

Dado que $\mathbb{S}^{n}$ é uma variedade Riemanniana completa, podemos estender o domínio da geodésica $\gamma$ para um intervalo $[0, b]$, onde $b>a$, e vamos supor que $\Gamma_{\gamma(t)}=\{e\}$, para todo $t \in[0, b]$. Definamos $\beta(t):=\psi \circ \gamma(t)$, assim, dado que $\psi$ é uma isometria, temos que $\beta(t)$ é também uma geodésica em $\mathbb{S}^{n}$. Seja $t \in[0, b]$, qualquer. Então existe $r_{t}$ tal que, da mesma maneira que mostramos para $x$

$$
\left.\eta^{-1} \circ \bar{\psi} \circ \pi\right|_{B_{r_{t}}(\gamma(t))}: B_{r_{t}}(\gamma(t)) \rightarrow B_{r_{t}}(\beta(t))
$$

é uma isometria. Assim, para cada $t \in[0, b]$ existe uma única isometria

$$
\psi_{t}: \mathbb{S}^{n} \rightarrow \mathbb{S}^{n}
$$

tal que $\psi_{t}(\gamma(t))=\beta(t)$ e

$$
\left.\psi_{t}\right|_{B_{r_{t}}(\gamma(t))}=\left.\eta^{-1} \circ \bar{\psi} \circ \pi\right|_{B_{r_{t}}(\gamma(t))} .
$$

Agora, $\left\{B_{r_{t}}(\gamma(t))\right\}_{t \in[0, b]}$ é uma cobertura por abertos de $\gamma([0, b])$. Então, pela compacidade de $\gamma([0, b])$, podemos reduzir a cobertura a uma finita $\left\{B_{r_{i}}\left(\gamma\left(t_{i}\right)\right)\right\}_{1 \leq i \leq k}$, onde podemos supor que $0 \leq t_{1}<t_{2}<\cdots<t_{k-1}<t_{k} \leq b$. Logo $x \in B_{r_{1}}\left(\gamma\left(t_{1}\right)\right)$, e portanto $\psi=\psi_{t_{1}}$. Dado que $B_{r_{1}}\left(\gamma\left(t_{1}\right)\right) \cap B_{r_{2}}\left(\gamma\left(t_{2}\right)\right)$ é não vazio, temos que $\psi_{t_{2}}=\psi_{t_{1}}$, logo $\psi_{t_{2}}=\psi$. Continuando de esta maneira, vamos obter que $\psi=\psi_{t_{i}}$, para todo $i=1,2, \ldots, k$. Portanto

$$
\eta \circ \psi(\gamma(t))=\bar{\psi} \circ \pi(\gamma(t))
$$


para todo $t \in[0, b]$.

Agora, suponhamos que $c>b$, e é tal que $\Gamma_{\gamma(c)} \neq\{e\}$ e $\Gamma_{\gamma(t)}=\{e\}$ para todo $0 \leq t<c$. Definamos $x_{1}:=\gamma(c)$. Afirmamos que $\eta \circ \psi\left(x_{1}\right)=\bar{\psi} \circ \pi\left(x_{1}\right)$. Com efeito, seja $\left\{t_{k}\right\}_{k \in \mathbb{N}}$ uma sequência contida em $[0, c)$, tal que $\lim _{j \rightarrow \infty} t_{j}=c$. Agora, para cada $j \in \mathbb{N}$, temos que, pelo provado acima, $\eta \circ \psi\left(\gamma\left(t_{j}\right)\right)=\bar{\psi} \circ \pi\left(\gamma\left(t_{j}\right)\right)$. Assim

$$
\begin{aligned}
\eta \circ \psi\left(x_{1}\right) & =\lim _{j \rightarrow \infty} \eta \circ \psi\left(\gamma\left(t_{j}\right)\right) \\
& =\lim _{j \rightarrow \infty} \bar{\psi} \circ \pi\left(\gamma\left(t_{j}\right)\right) \\
& =\bar{\psi} \circ \pi\left(\gamma\left(x_{1}\right)\right) .
\end{aligned}
$$

Logo $\eta \circ \psi(\gamma(t))=\bar{\psi} \circ \pi(\gamma(t))$, para todo $t \in[0, c]$.

Agora, dado que $\Gamma_{x_{1}} \neq\{e\}$, temos que $x_{1}$ não pertece a nenhuma bola da forma $B_{r_{t}}(\gamma(t))$, mencionadas anteriormente, para cada $t \in[0, c)$, dado que $\Gamma_{\gamma(t)}=\{e\}$. Seja $y_{1}=\psi\left(x_{1}\right)$. Para $x_{1}$, pelo Teorema 1.2.5, existe $r_{1}>0$ tal que $\pi$ induz uma isometria $\phi: B_{r}\left(x_{1}\right) / \Gamma_{x_{1}} \rightarrow B_{r}\left(x_{1}\right) / \Gamma$, para cada $r \leq r_{1}$. Da mesma maneira, para $y_{1}$ existe $s_{1}>0$ tal que $\eta$ induz uma isometria $\rho: B_{s}\left(y_{1}\right) / \tilde{\Gamma}_{y_{1}} \rightarrow B_{s}\left(y_{1}\right) / \tilde{\Gamma}$, para todo $s \leq s_{1}$. Definindo $\pi_{x_{1}}: B_{r_{1}}\left(x_{1}\right) \rightarrow B_{r_{1}}\left(x_{1}\right) / \Gamma_{x_{1}} \mathrm{e}$ $\eta_{y_{1}}: B_{s_{1}}\left(y_{1}\right) \rightarrow B_{s_{1}}\left(y_{1}\right) / \tilde{\Gamma}_{y_{1}}$, como as aplicações quocientes das ações de $\Gamma_{x_{1}}$ e $\tilde{\Gamma}_{y_{1}}$ sobre $B_{r_{1}}\left(x_{1}\right)$ e $B_{s_{1}}\left(y_{1}\right)$, respectivamente, temos os seguintes diagramas comutativos:

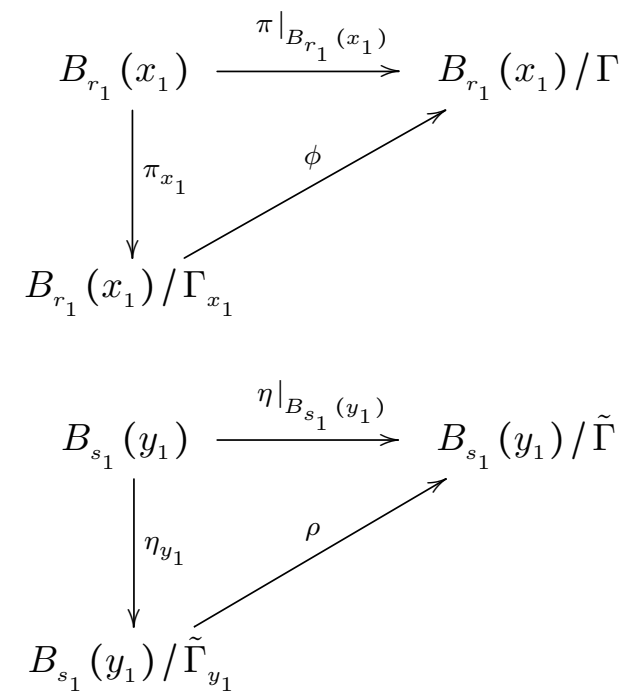

Seja $\bar{r}=\min \left\{r_{1}, s_{1}\right\}$. Então para todo $r \leq \bar{r}$, temos que

$$
\left.\left.\rho^{-1} \circ \bar{\psi} \circ \phi\right|_{\left.B_{r}\left(x_{1}\right)\right)}: B_{r}\left(x_{1}\right) / \Gamma_{x_{1}} \rightarrow B_{r}\left(y_{1}\right)\right) / \tilde{\Gamma}_{y_{1}}
$$

é uma isometria. Em especial, para $r<\bar{r}$, temos que

$$
\left.\rho^{-1} \circ \bar{\psi} \circ \phi\right|_{\mathbb{S}_{r}\left(x_{1}\right)}: \mathbb{S}_{r}\left(x_{1}\right) / \Gamma_{x_{1}} \rightarrow \mathbb{S}_{r}\left(y_{1}\right) / \tilde{\Gamma}_{y_{1}}
$$


é uma isometria. Fixemos $r<\bar{r}$. Dado que as esferas $\mathbb{S}_{r}\left(x_{1}\right)$ e $\mathbb{S}_{r}\left(y_{1}\right)$ são similares à esfera $\mathbb{S}^{n-1}$, podemos aplicar a hipótese de indução, i.e., existe uma isometria $\varphi_{r}: \mathbb{S}_{r}\left(x_{1}\right) \rightarrow \mathbb{S}_{r}\left(y_{1}\right)$, tal que

$$
\eta_{y_{1}} \circ \varphi_{r}=\left.\rho^{-1} \circ \bar{\psi} \circ \phi \circ \pi_{x_{1}}\right|_{\mathbb{S}_{r}\left(x_{1}\right)} .
$$

Então $\varphi_{r}$ é uma isometria entre $\left(\partial \bar{B}_{r}\left(x_{1}\right), \mathrm{g}_{0}\right)$ e $\left(\partial \bar{B}_{r}\left(y_{1}\right), \mathrm{g}_{0}\right)$, onde $\mathrm{g}_{0}$ é a métrica canónica em $\mathbb{S}^{n}$. Por [CLUV04] (Teorema 2.1), existe uma isometria $\tilde{\psi}: \bar{B}_{r}\left(x_{1}\right) \rightarrow \bar{B}_{r}\left(y_{1}\right)$ tal que $\tilde{\psi}$ é uma extensão de $\varphi_{r}$ e $\tilde{\psi}\left(x_{1}\right)=y_{1}$. Agora, por [Wol11] (Teorema 2.3.12), para $\tilde{\psi}$ existe uma única extensão a todo $\mathbb{S}^{n}$, que, por comodidade, chamaremos da mesma maneira como $\tilde{\psi}$. Então

$$
\left.\eta_{y_{1}} \circ \tilde{\psi}\right|_{\mathbb{S}_{r}\left(x_{1}\right)}=\eta_{y_{1}} \circ \varphi_{r}=\left.\rho^{-1} \circ \bar{\psi} \circ \phi \circ \pi_{x_{1}}\right|_{\mathbb{S}_{r}\left(x_{1}\right)} .
$$

Seja $s<\bar{r}$, mas diferente de $r$. Da mesma maneira que acontece para $r$, temos que existe uma isometria $\varphi_{s}: \mathbb{S}_{s}\left(x_{1}\right) \rightarrow \mathbb{S}_{s}\left(y_{1}\right)$, tal que

$$
\eta_{y_{1}} \circ \varphi_{s}=\left.\rho^{-1} \circ \bar{\psi} \circ \phi \circ \pi_{x_{1}}\right|_{\mathbb{S}_{r}\left(x_{1}\right)} .
$$

Afirmamos que $\left.\eta_{y_{1}} \circ \tilde{\psi}\right|_{\mathbb{S}_{s}\left(x_{1}\right)}=\eta_{y_{1}} \circ \varphi_{s}$. Vamos supor que isso não acontece, i.e., existe $x_{0} \in \mathbb{S}_{s}\left(x_{1}\right)$ tal que $\eta_{y_{1}} \circ \varphi_{s}\left(x_{0}\right) \neq \eta_{y_{1}} \circ \tilde{\psi}\left(x_{0}\right)$. Observemos que $B_{\bar{r}}\left(x_{1}\right)$ é uma bola normal de $x_{1}$. Assim, existe uma única geodésica $\alpha:[0, \bar{r}] \rightarrow \mathbb{S}^{n}$ tal que $\alpha(0)=x_{1}$ e $\alpha(s)=x_{0}$. Definindo $\tilde{\alpha}(t):=$ $\tilde{\psi}(\alpha(t))$, temos que $\tilde{\alpha}(t)$ é uma geodésica ligando y e $\psi\left(x_{0}\right)$. Agora, pela Proposição 1.8.1, temos que $\eta_{y_{1}}(\tilde{\alpha}(t))$ é uma geodésica métrica em $B_{\bar{r}}\left(y_{1}\right) / \tilde{\Gamma}_{y_{1}}$, e, por [Rat06] (Teorema 13.1.6), cada levantamento dela é único salvo ação por um elemento de $\tilde{\Gamma}_{y_{1}}$, i.e, cada levantamento de $\eta_{y_{1}}(\tilde{\alpha}(t))$ está determinado pela ação de $\bar{\Gamma}_{y_{1}}$ sobre $\dot{\tilde{\alpha}}(0)$. Dado que estamos supondo que $\eta_{y_{1}} \circ \varphi_{s}\left(x_{0}\right) \neq$ $\eta_{y_{1}} \circ \tilde{\psi}\left(x_{0}\right)$, então temos que $\varphi_{s}\left(x_{0}\right) \neq \tilde{\omega} \tilde{\psi}\left(x_{0}\right)$, para todo $\tilde{\omega}$ em $\tilde{\Gamma}_{y_{1}}$. Seja $\hat{\alpha}:[0, \bar{r}] \rightarrow \mathbb{S}^{n}$ a única geodésica tal que $\hat{\alpha}(0)=y_{1}$ e $\hat{\alpha}(s)=\varphi_{s}\left(x_{0}\right)$. Dado que $\left.\eta_{y_{1}} \circ \tilde{\psi}\right|_{\mathbb{S}_{r}(x)}=\eta_{y_{1}} \circ \varphi_{r}$, temos que $\eta_{y_{1}}(\hat{\alpha}(r))={ }_{y_{1}} \circ \tilde{\psi}(\alpha(r))=\tilde{\alpha}(r)$. Então $\dot{\hat{\alpha}}(0)$ e $\dot{\tilde{\alpha}}(0)$ estão na mesma $\tilde{\Gamma}_{y_{1}}$-órbita, e portanto $\hat{\alpha}(t)$ também é uma levantamento de $\eta_{y_{1}} \circ \tilde{\alpha}(t)$, mas isso contradiz o fato que $\varphi_{s}\left(x_{0}\right)$ e $\tilde{\psi}\left(x_{0}\right)$ não estão na mesma $\tilde{\Gamma}_{y_{1}}$-órbita. Logo temos que $\left.\eta_{y_{1}} \circ \tilde{\psi}\right|_{\mathbb{S}_{s}\left(x_{1}\right)}=\eta_{y_{1}} \circ \varphi_{s}$.

Portanto

$$
\left.\eta_{y_{1}} \circ \tilde{\psi}\right|_{B_{\bar{r}}\left(x_{1}\right)}=\left.\eta_{y_{1}} \circ \varphi_{s} \circ \tilde{\psi}\right|_{B_{\bar{r}}\left(x_{1}\right)}=\eta_{y_{1}} \circ \varphi_{r}=\left.\rho^{-1} \circ \bar{\psi} \circ \phi \circ \pi_{x_{1}}\right|_{B_{\bar{r}}\left(x_{1}\right)} .
$$

Sabemos que em $B_{\bar{r}}\left(x_{1}\right)$ existem infintos $\gamma(t)$, para $t \in[0, c)$. Assim, fixemos $\hat{t}$ tal que $\hat{t} \in[0, c)$ e $\gamma(\hat{t}) \in B_{\bar{r}}\left(x_{1}\right)$. Sabemos que $\Gamma_{\gamma(\hat{t})}$ é trivial, e portanto existe $\hat{r}>0$ tal que $\left.\psi\right|_{B_{\hat{r}}(\gamma(\hat{t}))}=\eta^{-1} \circ \bar{\psi} \circ$ $\left.\pi\right|_{B_{\hat{r}}(\gamma(\hat{t}))}$. Definindo $U:=B_{\hat{r}}(\gamma(\hat{t})) \cap B_{\bar{r}}\left(x_{1}\right)$, temos que $\gamma(\hat{t}) \in U$ e $\left.\psi\right|_{U}=\left.\eta^{-1} \circ \bar{\psi} \circ \pi\right|_{U}$. Seja 
$V:=\psi(U)$. Agora, sabemos que para todo $x \in U$ e todo $y \in V$ temos que $\Gamma_{x}=\{e\}$ e $\tilde{\Gamma}_{y}=\{\tilde{e}\}$. Logo $\left.\pi\right|_{U},\left.\pi_{x_{1}}\right|_{U},\left.\eta\right|_{V}$ e $\left.\eta_{y_{1}}\right|_{V}$ são isometrias. Assim

$$
\begin{aligned}
\left.\tilde{\psi}\right|_{U} & =\left.\eta_{y_{1}}^{-1} \circ \rho^{-1} \circ \bar{\psi} \circ \phi \circ \pi_{x_{1}}\right|_{U} \\
& =\left.\eta^{-1} \circ \bar{\psi} \circ \pi\right|_{U} \\
& =\left.\psi\right|_{U}
\end{aligned}
$$

pela equação (1.9) pois $\left.\eta\right|_{V}=\left.\phi \circ \eta_{1}\right|_{V}$ e $\left.\pi\right|_{U}=\left.\rho \circ \pi_{1}\right|_{U}$ dado que $\left.\psi\right|_{B_{\hat{r}}(\gamma(\hat{t}))}=\left.\eta^{-1} \circ \bar{\psi} \circ \pi\right|_{B_{\hat{r}}(\gamma(\hat{t}))}$.

Dado que $\tilde{\psi}$ e $\psi$ são isometrias de $\mathbb{S}^{n}$ e coincidem no aberto $U$ de $\mathbb{S}^{n}$, temos que $\tilde{\psi}=\psi$. Portanto

$$
\left.\eta \circ \psi\right|_{B_{\bar{r}}\left(x_{1}\right)}=\rho \circ \eta_{y_{1}} \circ \psi=\left.\bar{\psi} \circ \phi \circ \pi_{x_{1}}\right|_{B_{\bar{r}}\left(x_{1}\right)}=\left.\bar{\psi} \circ \pi\right|_{B_{\bar{r}}\left(x_{1}\right)}
$$

Logo $\eta \circ \psi(\gamma(t))=\bar{\psi} \circ \pi(\gamma(t))$, para todo $t \in[0, c+\bar{r})$, e por continuidade $\eta \circ \psi(\gamma(t))=$ $\bar{\psi} \circ \pi(\gamma(t))$, para todo $t \in[0, c+\bar{r}]$. Então podemos provar que $\eta \circ \psi(\gamma(t))=\bar{\psi} \circ \pi(\gamma(t))$, para todo $t \in[0,2 \pi]$. E assim temos que $\eta \circ \psi=\bar{\psi} \circ \pi$, que era nosso primeiro objetivo.

Agora, nosso segundo objetivo é provar que $\tilde{\Gamma}=\psi \Gamma \psi^{-1}$. Primeiro vamos demostrar que $\psi \Gamma \psi^{-1} \subset \tilde{\Gamma}$. Sejam $x_{0} \in \mathbb{S}^{n}$ e $y_{0}=\psi\left(x_{0}\right)$. Então

$$
\begin{aligned}
\psi \Gamma \psi^{-1}\left(y_{0}\right)=\left(\psi \Gamma \psi^{-1}\right) \psi\left(x_{0}\right) & =\psi \Gamma x_{0} & & \\
& =\tilde{\Gamma} \psi\left(x_{0}\right) & & \text { dado que } \eta \circ \psi=\bar{\psi} \circ \pi \\
& =\tilde{\Gamma} y_{0} & & \text { pois } y_{0}=\psi\left(x_{0}\right) .
\end{aligned}
$$

Assim, pela equação (1.10), temos que dado $\omega \in \Gamma$ e $y_{0} \in \mathbb{S}^{n}$, existe $\tilde{\omega}_{0} \in \tilde{\Gamma}$ tal que $\psi \circ \omega \circ \psi^{-1}\left(y_{0}\right)=$ $\tilde{\omega}_{0}\left(y_{0}\right)$. Então temos que $\eta \circ \psi \circ \omega \circ \psi^{-1}=\eta$. Podemos supor, sem perda de generalidade, que $y_{0}$ é tal que $\tilde{\Gamma}_{y_{0}}=\{\tilde{e}\}$. Logo, pela Proposição 1.2.2, existe $r>0$ tal que $\left\{\tilde{\omega} \in \tilde{\Gamma}: \tilde{\omega} B_{r}\left(y_{0}\right) \cap B_{r}\left(y_{0}\right) \neq\right.$ $\varnothing\}=\tilde{\Gamma}_{y_{0}}=\{\tilde{e}\}$ e $\tilde{\Gamma}_{y}=\{\tilde{e}\}$, para todo $y \in B_{r}\left(y_{0}\right)$. Além, neste caso, $\tilde{\Gamma}_{y_{0}} B_{r}\left(y_{0}\right)=B_{r}\left(y_{0}\right)$. Vamos provar que $\psi \circ \omega \circ \psi^{-1}=\tilde{\omega}_{0}$. Seja $y$ em $B_{r}\left(y_{0}\right)$, então é fácil ver que $\tilde{\Gamma} y \cap B_{r}\left(y_{0}\right)=\{y\}$. Agora

$$
\tilde{\omega}_{0}^{-1} \circ \psi \circ \omega \circ \psi^{-1}\left(y_{0}\right)=y_{0} \quad \text { e } \quad \eta \circ \tilde{\omega}_{0}^{-1} \circ \psi \circ \omega \circ \psi^{-1}(y)=\eta \circ \psi \circ \omega \circ \psi^{-1}(y)=\eta(y) \text {. }
$$

\section{Então}

$$
\tilde{\omega}_{0}^{-1} \circ \psi \circ \omega \circ \psi^{-1}(y) \in B_{r}\left(y_{0}\right) \text { e } \tilde{\omega}_{0}^{-1} \circ \psi \circ \omega \circ \psi^{-1}(y) \in \tilde{\Gamma} y \text {, respectivamente. }
$$


Logo $\tilde{\omega}_{0}^{-1} \circ \psi \circ \omega \circ \psi^{-1}(y) \in \tilde{\Gamma} y \cap B_{r}\left(y_{0}\right)=\{y\}$. Dado que $y$ é qualquer ponto de $B_{r}\left(y_{0}\right)$, temos que $\psi \circ \omega \circ \psi^{-1}$ e $\tilde{\omega}_{0}$ coincidem em $B_{r}\left(y_{0}\right)$. Como $\psi \circ \omega \circ \psi^{-1}$ e $\tilde{\omega}_{0}$ são isometrias de $\mathbb{S}^{n}$, então $\psi \circ \omega \circ \psi^{-1}=\tilde{\omega}_{0}$. Por tanto $\psi \Gamma \psi^{-1} \subset \tilde{\Gamma}$.

Agora, fazendo uma prova similar, podemos provar que $\tilde{\Gamma} \subset \psi \Gamma \psi^{-1}$. Então $\tilde{\Gamma}=\psi \Gamma \psi^{-1}$.

Observação 1.8.2 Na prova da Proposição 1.8.2 demonstramos que se

$$
\left.\rho^{-1} \circ \bar{\psi} \circ \phi\right|_{B_{\bar{r}}\left(x_{1}\right)}: B_{\bar{r}}\left(x_{1}\right) / \Gamma_{x_{1}} \rightarrow B_{\bar{r}}\left(y_{1}\right) / \tilde{\Gamma}_{y_{1}}
$$

é uma isometria de espaços métricos, então existe uma isometria $\psi: \mathbb{S}^{n} \rightarrow \mathbb{S}^{n}$, tal que

$$
\left.\eta_{y_{1}} \circ \psi\right|_{B_{\bar{r}}\left(x_{1}\right)}=\left.\rho^{-1} \circ \bar{\psi} \circ \phi \circ \pi_{x_{1}}\right|_{B_{\bar{r}}\left(x_{1}\right)} .
$$

Portanto, podemos concluir que se $B_{r}(0)$ é uma bola aberta num espaço Euclidiano $\mathbb{V}, \Gamma$ e $\tilde{\Gamma}$ são dois grupos finitos de $\mathbb{O}(\mathbb{V})$ e $\bar{\psi}: B_{r}(0) / \Gamma \rightarrow B_{r}(0) / \tilde{\Gamma}$ uma isometria de espaços métricos, então existe uma isometria $\psi: B_{r}(0) \rightarrow B_{r}(0)$, tal que

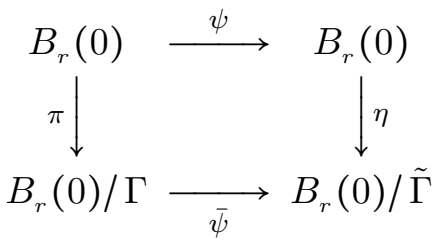

onde $\pi$ e $\eta$ são as aplicações quocientes respectivas. Além disso, também podemos concluir que $\psi \Gamma \psi^{-1}=\tilde{\Gamma}$.

Observação 1.8.3 Uma propriedade interessante que possui um orbifold Riemannino é que é um espaço métrico (vide [Bor92] ou [BH99]). Agora, [LT10] dá uma definição alternativa, que é equivalente à definição padrão de um orbifold Riemanniano:

Definiçẫo 1.8.1 Um espaço métrico $X$ é chamado de orbifold Riemanniano se cada $x \in X$ admite uma vizinhança aberta $x \in U \subset X$ tal que $U$ é isométrica ao quociente $M / \Gamma$, onde $(M, g)$ é uma variedade Riemanniana e $\Gamma$ é um subgrupo finito de $\operatorname{Iso}(M, g)$.

A seguinte proposição mostra que um orbifold Riemanniano é localmente representado de uma única maneira. 
Proposição 1.8.3 Sejam $M$ e $N$ duas variedades Riemannianas conexas. Vamos supor que $\Gamma<$ Iso $(M), \tilde{\Gamma}<$ Iso $(N)$ e que $\Gamma$ e $\tilde{\Gamma}$ são discretos e agem propriamente sobre $M$ e $N$, respectivamente. Se $\bar{\psi}: M / \Gamma \rightarrow N / \tilde{\Gamma}$ é uma isometria de espaços métricos, então existe uma isometria definida localmente $\psi: M \rightarrow N$ que induz $\bar{\psi}$, i.e., se definimos $\pi: M \rightarrow M / \Gamma$ e $\eta: N \rightarrow N / \tilde{\Gamma}$ as aplicaçôes quocientes respectivas, e sendo $x \in M$ e $y \in N$ tais que $y \in \eta^{-1}(\bar{\psi}(\pi(x)))$, então existe $r>0$ e uma isometria Riemanniana

$$
\psi: B_{r}(x) \rightarrow B_{r}(y)
$$

tal que o seguinte diagrama é comutativo

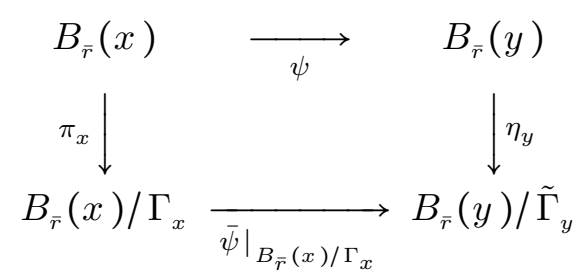

Além disso, temos que $\psi \Gamma_{x} \psi^{-1}=\tilde{\Gamma}_{y}$, onde $\pi_{x}$ e $\eta_{y}$ são as aplicaçôes quocientes respectivas.

Demonstração. Sejam $\bar{x} \in M / \Gamma, \bar{y}=\bar{\psi}(\bar{x})$ e $\pi: M \rightarrow M / \Gamma, \eta: N \rightarrow N / \tilde{\Gamma}$ as aplicações quocientes respectivas. Fixemos $x \in \pi^{-1}(\bar{x})$. Pelo Teorema 1.2.5 existe $\tilde{r}>0$ tal que $\pi$ induz uma isometria $\phi: B_{r}(x) / \Gamma_{x} \rightarrow B_{r}(x) / \Gamma$, para todo $r \leq \tilde{r}$. Fixemos $y \in \eta^{-1}(\bar{y})$. Lembremos que pela Proposição 1.8.2 temos que $y$ é um ponto regular em $N$ se e somente se $x$ é um ponto regular em $M$. Assim, novamente pelo Teorema 1.2.5, existe $\tilde{s}>0$ tal que $\eta$ induz uma isometria $\rho: B_{s}(y) / \tilde{\Gamma}_{y} \rightarrow$ $B_{s}(y) / \tilde{\Gamma}$, para todo $s \leq \tilde{s}$. Seja $\bar{r}=\min \{\tilde{r}, \tilde{s}\}$, e vamos supor, sem perda de generalidade, que $B_{\bar{r}}(x)$ e $B_{\bar{r}}(y)$ são bolas normais para $x$ e $y$, respectivamente. Agora, não é difícil ver que $B_{\bar{r}}(x) / \Gamma_{x} \subset$ $B_{\bar{r}}(x) / \Gamma$ e que, dado que $\pi$ é uma aplicação aberta, $B_{\bar{r}}(x) / \Gamma=B_{\bar{r}}(\Gamma x)$ é abierto em $M / \Gamma$. Portanto, dado que $\phi$ é uma isometria, temos que $B_{\bar{r}}(x) / \Gamma_{x}$ é aberto em $M / \Gamma$. De igual maneira podemos provar que $B_{\bar{r}}(y) / \tilde{\Gamma}_{y}$ é aberto em $N / \tilde{\Gamma}$.

Então

$$
\left.\bar{\psi}\right|_{B_{\bar{r}}(x) / \Gamma_{x}}: B_{\bar{r}}(x) / \Gamma_{x} \rightarrow B_{\bar{r}}(y) / \tilde{\Gamma}_{y}
$$

é uma isometria.

Sejam $\pi_{x}: B_{\bar{r}}(x) \rightarrow B_{\bar{r}}(x) / \Gamma_{x}$ e $\eta_{y}: B_{\bar{r}}(y) \rightarrow B_{\bar{r}}(y) / \tilde{\Gamma}_{y}$ as aplicações quocientes respectivas. Sabemos que $\Gamma_{x}$ age sobre $B_{\bar{r}}(0) \subset T_{x} M$, e que ação é definida da seguinte maneira

$$
\begin{aligned}
\mu: \Gamma \times B_{\bar{r}}(0) & \longrightarrow B_{\bar{r}}(0) \\
(\omega, u) & \longmapsto
\end{aligned}
$$


De maneira similar está definida a ação de $\tilde{\Gamma}_{y}$ sobre $B_{\bar{r}}(0) \subset T_{y} N$. Definamos então

$$
D \pi_{x}: B_{\bar{r}}(0) \rightarrow B_{\bar{r}}(0) / \Gamma_{x} \text { e } D \eta_{y}: B_{\bar{r}}(0) \rightarrow B_{\bar{r}}(0) / \tilde{\Gamma}_{y}
$$

como as aplicações quocientes respectivas das ações antes mencionadas.

Observemos que

$$
\begin{aligned}
& \omega \exp _{x}(u)=\exp _{x}\left((d \omega)_{x} u\right) ; \text { para todo } u \in B_{\bar{r}}(0) \subset T_{x} M \text { e para todo } \omega \in \Gamma_{x} \\
& \tilde{\omega} \exp _{y}(v)=\exp _{x}\left((d \tilde{\omega})_{y} v\right) ; \text { para todo } v \in B_{\bar{r}}(0) \subset T_{y} N \text { e para todo } \tilde{\omega} \in \tilde{\Gamma}_{y} .
\end{aligned}
$$

Seja $x_{1} \in B_{\bar{r}}(x)$ qualquer, então existe um único $u_{1} \in B_{\bar{r}}(0) \subset T_{x} M$ tal que $\exp _{x}\left(u_{1}\right)=x_{1}$. Seja $y_{1} \in B_{\bar{r}}(y)$ tal que $\eta_{y}\left(y_{1}\right)=\bar{\psi}\left(\Gamma_{x} x_{1}\right)$, então sabemos que existe um único $v_{1} \in B_{\bar{r}}(0) \subset T_{x} N$ tal que $\exp _{y}\left(v_{1}\right)=y_{1}$. Assim, vamos definir a seguinte aplicação

$$
\begin{aligned}
& (D \bar{\psi})_{\bar{x}}: \quad B_{\bar{r}}(0) / \Gamma_{x} \longrightarrow B_{\bar{r}}(0) / \tilde{\Gamma}_{y} \\
& \Gamma_{x} u_{1} \quad \longmapsto \quad \tilde{\Gamma}_{y} v_{1} .
\end{aligned}
$$

Dado que $\exp _{x}: B_{\bar{r}}(0) \subset T_{x} M \rightarrow B_{\bar{r}}(x)$ e $\exp _{y}: B_{\bar{r}}(0) \subset T_{y} N \rightarrow B_{\bar{r}}(y)$ são difeomorfismos, vamos induzir a métrica $\mathrm{g}_{0}$ de $B_{\bar{r}}(0) \subset T_{x} M$ sobre $B_{\bar{r}}(x) \subset M$ e a métrica $\mathrm{g}_{0}$ de $B_{\bar{r}}(0) \subset T_{y} N$ sobre $B_{\bar{r}}(y) \subset N$, e assim as aplicações $\exp _{x}$ e exp viram isometrias Riemannianas.

Assim, afirmamos que

$$
(D \bar{\psi})_{\bar{x}}:\left(B_{\bar{r}}(0), \mathrm{g}_{0}\right) / \Gamma_{x} \longrightarrow\left(B_{\bar{r}}(0), \mathrm{g}_{0}\right) / \tilde{\Gamma}_{y}
$$

é uma isometria de espaços métricos. Com efeito, sejam $x_{i}=\exp _{x}\left(u_{i}\right)$ e $y_{i}=\exp _{y}\left(v_{i}\right)$, onde $u_{i} \in B_{\bar{r}}(0) \subset T_{x} M$ e $v_{i} \in B_{\bar{r}}(0) \subset T_{y} N$, para $i=1,2$. Vamos supor que $\eta_{y}\left(y_{i}\right)=\bar{\psi}\left(\Gamma_{x} x_{i}\right)$, para 
$i=1,2$, respectivamente. Então

$$
\begin{aligned}
d_{\tilde{\Gamma}_{y}}\left((D \bar{\psi})_{\bar{x}} \Gamma_{x} u_{1},(D \bar{\psi})_{\bar{x}} \Gamma_{x} u_{2}\right) & =d_{\tilde{\Gamma}_{y}}\left(\tilde{\Gamma}_{y} v_{1}, \tilde{\Gamma}_{y} v_{2}\right) & & \text { pela definição de }(D \bar{\psi})_{x} \\
& =\inf \left\{d\left(v_{1},(d \tilde{\omega})_{y} v_{2}\right): \tilde{\omega} \in \tilde{\Gamma}_{y}\right\} & & \text { pela definição de } d_{\tilde{\Gamma}_{y}} \\
& =\inf \left\{d\left(y_{1}, \tilde{\omega} y_{2}\right): \tilde{\omega} \in \tilde{\Gamma}_{y}\right\} & & \text { de }(1.11) \text { e exp } \operatorname{é}_{y} \text { isometria } \\
& =d_{\tilde{\Gamma}_{y}}\left(\tilde{\Gamma}_{y} y_{1}, \tilde{\Gamma}_{y} y_{2}\right) & & \text { pela definição de } d_{\tilde{\Gamma}_{y}} \\
& =d_{\Gamma_{x}}\left(\Gamma_{x} x_{1}, \Gamma_{x} x_{2}\right) & & \text { pois } \bar{\psi} \text { é uma isometria } \\
& =i n f\left\{d\left(x_{1}, \omega x_{2}\right): \omega \in \Gamma_{x}\right\} & & \text { pela definição de } d_{\Gamma_{x}} \\
& =i n f\left\{d\left(u_{1},(d \omega)_{x}\left(u_{2}\right)\right): \omega \in \Gamma_{x}\right\} & & \text { de (1.11) e exp } x_{x} \text { é isometria } \\
& =d_{\Gamma_{x}}\left(u_{1}, u_{2}\right) & & \text { pela definição de } d_{\Gamma_{x}} .
\end{aligned}
$$

Então, efetivamente, $(D \bar{\psi})_{\bar{x}}$ é uma isometria de espaços métricos.

Agora, pela Observação 1.8.2, existe uma isometria $(D \psi)_{x}: B_{\bar{r}}(0) \subset T_{x} M \rightarrow B_{\bar{r}}(0) \subset T_{y} N$ tal que o seguinte diagrama é comutativo

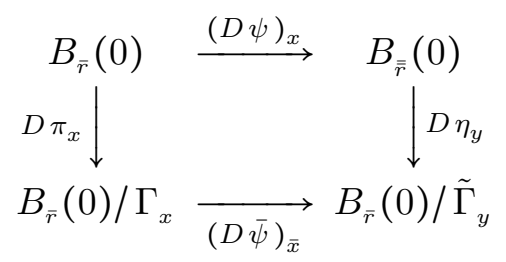

e tal que $(D \psi)_{x} \Gamma_{x}(D \psi)_{x}^{-1}=\tilde{\Gamma}_{y}$.

Pela equação (1.11) temos que $\exp _{x}: B_{\bar{r}}(0) \subset T_{x} M \rightarrow B_{\bar{r}}(x)$ e $\exp _{y}: B_{\bar{r}}(0) \subset T_{y} N \rightarrow B_{\bar{r}}(y)$ são aplicações $\Gamma_{x}$-invariante e $\tilde{\Gamma}_{y}$-invariante, respectivamente. Assim, definamos o seguinte difeomorfismo

$$
\begin{array}{rlc}
\psi: B_{\bar{r}}(x) & \longrightarrow & B_{\bar{r}}(y) \\
\cdot & \longmapsto & \exp _{y} \circ(D \psi)_{x} \circ \exp _{x}^{-1}(\cdot)
\end{array}
$$

Seja $\omega$ qualquer elemento de $\Gamma_{x}$, então existe um único $\tilde{\omega}$ em $\tilde{\Gamma}_{y}$ tal que

$$
(D \psi)_{x} \circ(d \omega)_{x} \circ(D \psi)_{x}^{-1}=(d \tilde{\omega})_{y} .
$$


Assim

$$
\begin{aligned}
\psi(\omega(\cdot)) & =\exp _{y} \circ(D \psi)_{x} \circ \exp _{x}^{-1}(\omega(\cdot)) & & \text { pela definição de } \psi \\
& =\exp _{y} \circ(D \psi)_{x} \circ(d \omega)_{x} \circ \exp _{x}^{-1}(\cdot) & & \text { pela equação }(1.11) \\
& =\exp _{y} \circ(d \tilde{\omega})_{y} \circ(D \psi)_{x} \circ \exp _{x}^{-1}(\cdot) & & \text { pois }(D \psi)_{x} \circ(d \omega)_{x} \circ(D \psi)_{x}^{-1}=(d \tilde{\omega})_{y} \\
& =\tilde{\omega}\left(\exp _{y} \circ(D \psi)_{x} \circ \exp _{x}^{-1}(\cdot)\right) & & \text { pela equação }(1.11) \\
& =\tilde{\omega}(\psi(\cdot)) & & \text { pela definição de } \psi .
\end{aligned}
$$

Logo $\psi$ é um difeomorfismo $\Gamma_{x}-\tilde{\Gamma}_{y}-$ invariante. Dado isto, podemos induzir a seguinte aplicação

$$
\begin{aligned}
\bar{\phi}: B_{\bar{r}}(x) / \Gamma_{x} & \longrightarrow B_{\bar{r}}(y) / \tilde{\Gamma}_{y} \\
\Gamma_{x} \cdot & \longmapsto \Gamma_{y} \psi(\cdot) .
\end{aligned}
$$

Agora afirmamos que $\bar{\phi}$ é um homeomorfismo. Primeiro observemos que $\bar{\phi}$ está bem definida pois $\psi$ é uma bijeção $\Gamma_{x}-\tilde{\Gamma}_{y}-$ invariante. Além disso, pela definição de $\bar{\phi}$, temos que o seguinte diagrama é comutativo

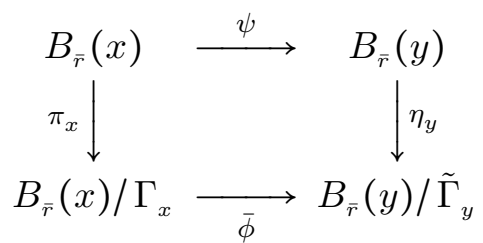

Dado que $\bar{\phi}$ é bem definida, temos que ela é injetora. $\bar{\phi}$ é sobrejetora pois $\psi$ é sobrejetora. Dado que $\eta \circ \psi$ é aberta e contínua e que o diagrama acima é comutativo, temos que $\bar{\phi}$ é aberta e contínua, respectivamente. Portanto $\bar{\phi}$ é homeomorfismo.

Agora vamos provar que $\left.\bar{\psi}\right|_{B_{\bar{r}}(x) / \Gamma_{x}}=\bar{\phi}$. Seja $\gamma:[0, \bar{r}) \rightarrow B_{\bar{r}}(x)$ uma geodésica tal que $\gamma(0)=x$, $\dot{\gamma}(0)=u$ e $|u|=1$. Agora, $\gamma$ é minimizante pois $B_{\bar{r}}(x)$ é uma bola normal de $x$. Observemos que para todo $t \in[0, \bar{r})$ temos, pela equação $(1.11)$, que $\Gamma_{x} \gamma(t)=\Gamma_{x} \exp _{x}(t u)=\left\{\exp _{x}\left(t(d \omega)_{x} u\right)\right.$ : $\left.\omega \in \Gamma_{x}\right\}$. Agora, pela Proposição 1.8.1, temos que $\pi_{x} \circ \gamma(t)$ é uma geodésica métrica em $B_{\bar{r}}(x) / \Gamma_{x}$, e $\pi_{x} \circ \gamma(0)=\pi_{x}(x)$. Portanto, dado que $\bar{\psi}$ é uma isometria de espaços métricos, temos que $\bar{\psi} \circ \pi_{x} \circ \gamma(t)$ é uma geodésica métrica em $B_{\bar{r}}(y) / \tilde{\Gamma}_{y}, \operatorname{com} \bar{\psi} \circ \pi_{x} \circ \gamma(0)=\eta_{y}(y)$. Por [Rat06] (Teorema 13.1.6) existe uma única geodésica minimizante, salvo ação de $\tilde{\Gamma}_{y}$ sobre $y, \tilde{\beta}:[0, \bar{r}) \rightarrow B_{\bar{r}}(y)$, tal que

$$
\tilde{\beta}(0)=y \quad \text { e } \quad \eta_{y} \circ \tilde{\beta}(t)=\bar{\psi} \circ \pi_{x} \circ \gamma(t) .
$$

Supomos que $\dot{\tilde{\beta}}(0)=v$ e que $|v|=1$. Então, pela equação $(1.11)$, para cada $t \in[0, \bar{r})$, temos que $\tilde{\Gamma}_{y} \tilde{\beta}(t)=\tilde{\Gamma}_{y} \exp _{y}(t v)=\left\{\exp _{y}\left(t(d \tilde{\omega})_{y} v\right): \tilde{\omega} \in \tilde{\Gamma}_{y}\right\}$. 
Agora

$$
\begin{aligned}
\bar{\phi} \circ \pi_{x} \circ \gamma(t) & =\eta_{y} \circ \psi \circ \gamma(t) & & \text { pois } \bar{\phi} \circ \pi_{x}=\eta_{y} \circ \psi \\
& =\eta_{y} \circ \exp _{y} \circ(D \psi)_{x} \circ \exp _{x}^{-1} \circ \gamma(t) & & \text { pela definição de } \psi \\
& =\eta_{y} \circ \exp _{y} \circ(D \psi)_{x}(t u) & & \text { dado que } \gamma(t)=\exp _{x}(t u) \\
& =\eta_{y} \circ \exp _{y}\left(t(D \psi)_{x}(u)\right) & & \text { pois }(D \psi)_{x} \text { é linear. }
\end{aligned}
$$

Por outra parte

$$
\begin{aligned}
D \eta_{y} \circ(D \psi)_{x}(t u) & =(D \bar{\psi})_{\bar{x}} \circ D \pi_{x}(t u) & & \text { pois } D \eta_{y} \circ(D \psi)_{x}=(D \bar{\psi})_{\bar{x}} \circ D \pi_{x} \\
& =D \eta_{y}(t v) & & \text { por }(1.12) \text { e a definição de }(D \bar{\psi})_{\bar{x}} .
\end{aligned}
$$

Então temos, pela equação (1.14), que $\psi(u)$ e $v$ estão na mesma $\tilde{\Gamma}_{y}$-órbita. Portanto, aplicando isto na equação (1.13), obtemos que

$$
\bar{\phi} \circ \pi_{x} \circ \gamma(t)=\eta_{y} \circ \exp _{y}(t \psi(u))=\eta_{y} \circ \exp _{y}(t v)=\bar{\psi} \circ \pi_{x} \circ \gamma(t)
$$

Assim, dado que $\gamma$ é qualquer geodésica em $B_{\bar{r}}(x) \operatorname{com} \gamma(0)=x$ e $B_{\bar{r}}(x)$ é uma bola normal de $x$, temos que $\left.\bar{\psi}\right|_{B_{\bar{r}}(x) / \Gamma_{x}}=\bar{\phi}$, que era nosso primeiro objetivo. Além disso, temos que o seguinte diagrama é comutativo

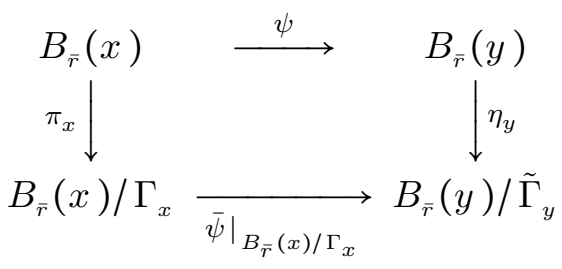

Agora vamos provar que $\psi: B_{\bar{r}}(x) \rightarrow B_{\bar{r}}(y)$ é uma isometria de variedades Riemannianas. Seja $x_{1} \in B_{\bar{r}}(x)$ tal que $\Gamma_{x_{1}}$ é trivial, e seja $y_{1}=\psi\left(x_{1}\right)$. Então $\bar{\phi} \circ \pi_{x}\left(x_{1}\right)=\eta_{y}\left(y_{1}\right)$, e, pela Proposição 1.8.2, $\tilde{\Gamma}_{y_{1}}$ é trivial. Assim, pelo Teorema 1.2.5, existe um $\bar{s}>0$ tal que

$$
\left.\pi\right|_{B_{\bar{s}}\left(x_{1}\right)}: B_{\bar{s}}\left(x_{1}\right) \rightarrow B_{\bar{s}}\left(x_{1}\right) / \Gamma \quad \text { e }\left.\quad \eta\right|_{B_{\bar{s}}\left(y_{1}\right)}: B_{\bar{s}}\left(y_{1}\right) \rightarrow B_{\bar{s}}\left(y_{1}\right) / \tilde{\Gamma}
$$

são isometrias. Neste caso $\bar{s}<\bar{r}$, e podemos supor que $B_{\bar{s}}\left(x_{1}\right) \subset B_{\bar{r}}\left(x_{1}\right)$. Portanto $B_{\bar{s}}\left(y_{1}\right) \subset$ $B_{\bar{r}}\left(y_{1}\right),\left.\pi_{x}\right|_{B_{\bar{s}}\left(x_{1}\right)}=\left.\pi\right|_{B_{\bar{s}}\left(x_{1}\right)}$ e $\left.\eta\right|_{B_{\bar{s}}\left(y_{1}\right)}=\left.\eta_{y}\right|_{B_{\bar{s}}\left(y_{1}\right)}$. Logo

$$
\left.\eta^{-1} \circ \bar{\psi} \circ \pi\right|_{B_{\bar{s}}\left(x_{1}\right)}: B_{\bar{s}}\left(x_{1}\right) \rightarrow B_{\bar{s}}\left(y_{1}\right)
$$


é uma isometria de espaços métricos. E, dado que $\left.\eta^{-1} \circ \bar{\psi} \circ \pi\right|_{B_{\bar{s}}\left(x_{1}\right)}=\left.\psi\right|_{B_{\bar{s}}\left(x_{1}\right)}$, temos então que

$$
\left.\psi\right|_{B_{\bar{s}}\left(x_{1}\right)}: B_{\bar{s}}\left(x_{1}\right) \longrightarrow B_{\bar{s}}\left(y_{1}\right)
$$

é uma isometria de espaços métricos. Agora, pelo Teorema de Myers-Steenrod, temos que $\left.\psi\right|_{B_{\bar{s}}\left(x_{1}\right)}$ torna-se uma isometria entre variedades Riemannianas. Denotemos por $\Omega\left(B_{\bar{r}}(x)\right)$ ao conjunto dos pontos regulares em $B_{\bar{r}}(x)$. Então, dado que $x_{1}$ é qualquer elemento de $\Omega\left(B_{\bar{r}}(x)\right)$, temos que $\psi$ é uma isometria Riemanniana local em $\Omega\left(B_{\bar{r}}(x)\right)$. Agora, por [Rat06] (Teorema 13.2.4), temos que $\Omega\left(B_{\bar{r}}(x)\right)$ é um conjunto aberto e denso em $B_{\bar{r}}(x)$, então $\left.\psi\right|_{B_{\bar{r}}(x)}$ é uma isometria Riemanniana local. Por outro lado, sabemos que $\left.\psi\right|_{B_{\bar{r}}(x)}$ é bijetora, portanto $\left.\psi\right|_{B_{\bar{r}}(x)}$ é uma isometria Riemanniana, como desejávamos provar.

Agora vamos provar que $\psi \Gamma_{x} \psi^{-1}=\tilde{\Gamma}_{y}$. Primero vamos demonstrar que $\psi \Gamma_{x} \psi^{-1} \subset \tilde{\Gamma}_{y}$. Dado que $\bar{\psi} \circ \pi_{x}=\eta_{y} \circ \psi$ e fazendo uma ideia análoga como na equação (1.10), podemos concluir que para cada $y_{0} \in B_{y}$ e para cada $\omega \in \Gamma_{x}$, existe $\tilde{\omega}_{0} \in \tilde{\Gamma}_{y}$ tal que $\psi \circ \omega \circ \psi^{-1}\left(y_{0}\right)=\tilde{\omega}_{0}\left(y_{0}\right)$. Então queremos provar que $\psi \circ \omega \circ \psi^{-1}=\left.\tilde{\omega}_{0}\right|_{B_{\bar{r}}(y)}$. Seja $D$ é um domínio fundamental da ação de $\tilde{\Gamma}_{y}$ sobre $B_{\bar{r}}(y)$, e vamos supor que $y_{0} \in D$. Sabemos que $D$ é um subconjunto aberto de $B_{\bar{r}}(y)$, então $\tilde{\omega}_{0}(D)$ também é aberto em $B_{\bar{r}}(y)$. Agora, $\psi \circ \omega \circ \psi^{-1}$ é uma isometria, em especial é uma aplicação contínua, então existe $\delta>0$ tal que $B_{\delta}\left(y_{0}\right) \subset D$ e $\psi \circ \omega \circ \psi^{-1}\left(B_{\delta}\left(y_{0}\right)\right) \subset \tilde{\omega}_{0} D$. Seja $y_{1} \in B_{\delta}\left(y_{0}\right)$. Então para $y_{1}$, igual que mostramos para $y_{0}$, existe $\tilde{\omega}_{1} \in \tilde{\Gamma}_{y}$ tal que $\psi \circ \omega \circ \psi^{-1}\left(y_{1}\right)=\tilde{\omega}_{1}\left(y_{1}\right)$. Agora, $y_{1} \in D$, pois $B_{\delta}\left(y_{0}\right) \subset D$. Portanto $\psi \circ \omega \circ \psi^{-1}\left(B_{\delta}\left(y_{0}\right)\right) \subset \tilde{\omega}_{1}(D)$. Assim $\tilde{\omega}_{1}^{-1} \circ \psi \circ \omega \circ \psi^{-1}\left(B_{\delta}\left(y_{0}\right)\right) \subset D \mathrm{e}$ $\tilde{\omega}_{0}^{-1} \circ \psi \circ \omega \circ \psi^{-1}\left(B_{\delta}\left(y_{0}\right)\right) \subset D$. Dado que $\psi \circ \omega \circ \psi^{-1}\left(B_{\delta}\left(y_{0}\right)\right)$ é um subconjunto aberto não vazio de $B_{\bar{r}}(y)$ e a familia $\left\{\tilde{\omega} D: \tilde{\omega} \in \tilde{\Gamma}_{y}\right\}$ é disjunta dois a dois, pois $D$ é um domínio fundamental, temos que $\tilde{\omega}_{1}=\tilde{\omega}_{0}$. Dado que $y_{1}$ é qualquer ponto de $B_{\delta}\left(y_{0}\right)$, então temos que $\left.\psi \circ \omega \circ \psi^{-1}\right|_{B_{\delta}\left(y_{0}\right)}=\left.\tilde{\omega}_{0}\right|_{B_{\delta}\left(y_{0}\right)}$. E, dado que $\psi \Gamma_{x} \psi^{-1}$ e $\tilde{\omega}_{0}$ são isometrias de $B_{\bar{r}}(y)$, temos que $\psi \circ \omega \circ \psi^{-1}=\left.\tilde{\omega}_{0}\right|_{B_{\bar{r}}(y)}$. Então, dado que $B_{\bar{r}}(y)$ é um aberto conexo de $N$, então $\psi \circ \omega \circ \psi^{-1}=\tilde{\omega}_{0}$. Portanto $\psi \Gamma_{x} \psi^{-1} \subset \tilde{\Gamma}_{y}$. Da mesma maneira podemos provar que $\psi^{-1} \tilde{\Gamma}_{y} \psi \subset \Gamma_{x}$. Logo $\psi \Gamma_{x} \psi^{-1}=\tilde{\Gamma}_{y}$. E assim temos toda prova da proposição. 


\section{CAPÍTULO 2}

\section{EXISTÊNCIA DE GEODÉSICAS FECHADAS NUM ORBIFOLD \\ RIEMANNIANO}

«Pessoas normais falam sobre coisas, pessoas inteligentes falam sobre idéias, pessoas mesquinhas falam sobre pessoas.»

-Platão

Neste capítulo vamos falar de alguns resultados sobre existência de geodésicas fechadas em orbifolds Riemannianos compactos.

Na Seção 1, daremos a definição de uma geodésica fechada num orbifold Riemanniano. Em especial, vamos expor a definição de uma geodésica fechada num good orbifold Riemanniano (definição que é mais fácil de entender que a definição num orbifold Riemanniano que não é de tipo good), e as geodédicas fechadas num good orbifold Riemanniano são nosso objeto de estudo no Capítulo 3 . A seção está baseada em [AJ11], [Dra11] e [Dra14]. 
Na seguinte seção, daremos os conceitos de ações cocompactas, função deslocamento e uma clasificação de isometrias em três tipos: elíptico, hiperbólico e parabólico. Destes três tipos de isometrias, para nós serão úteis os dois primeiros tipos, e, em tais casos, uma isometria será chamada semi-simples. A seção é inspirada em [BH99].

Na Seção 3 aparece o Teorema 2.3.1; primeiro resultado de existência de geodésica fechada num good orbifold Riemanniano compacto com algumas condições adicionais. Tal resultado é baseado no Processo de Duplo Encurtamento, conceito que também é dado nesta seção. Os resultados desta seção são citados de [AJ11].

Finalmente, na Seção 4, vamos expor o Teorema 2.4.1; resultado que garante a existência de uma geodésica fechada não trivial num good orbifold Riemanniano compacto $M / \Gamma$, onde $\Gamma$ é finito ou possui um elemento de tipo hiperbólico. O resultado é muito importante para nós, pois ele será usado nos resultados principais do Capítulo 3. Também, nesta Seção, discutiremos alguns resultados de existência de geodésicas fechadas a partir do conceito de curvatura seccional num orbifold Riemanniano. A seção está baseada em [Dra14].

\subsection{Geodésica Fechada num Orbifold Riemanniano}

Definição 2.1.1 Seja $M / \mathcal{P}$ um orbifold Riemanniano (vide definição 1.7.1). Uma geodésica fechada em $M / \mathcal{P}$ é definida da seguinte maneira

1. uma sequência $0=t_{0} \leq t_{1} \leq \ldots \leq t_{n-1} \leq t_{n}=1$;

2. uma sequência de segmentos de geodésica não triviais $\alpha_{i}:\left[t_{t-1}, t_{i}\right] \rightarrow M$, para $i=1,2, \ldots, n$;

3. uma sequência $\omega_{i} \in \mathcal{P}$, para $i=1,2, \ldots, n$, onde cada $\omega_{i}$ está definida numa vizinhança aberta de $\alpha_{i}\left(t_{i}\right), U_{i} \subset M$, tal que

$$
\omega_{i}\left(\alpha_{i}\left(t_{i}\right)\right)=\alpha_{i}\left(t_{i+1}\right) \text { e d } \omega_{i} \dot{\alpha}_{i}\left(t_{i}\right)=\dot{\alpha}_{i}\left(t_{i+1}\right), \text { para } 1 \leq i \leq n-1
$$

$e$

$$
\omega_{n} \alpha_{n}\left(t_{n}\right)=\alpha_{1}(0) \text { e } d \omega_{n} \dot{\alpha}_{i}\left(t_{n}\right)=\dot{\alpha}_{1}(0)
$$

Neste caso, uma geodésica fechada em $M / \mathcal{P}$ é denotada por $\left\{\left(\omega_{i}, \alpha_{i}\right)\right\}_{1 \leq i \leq n}$. 
Observação 2.1.1 Um good orbifold Riemanniano X será denotado, pela Observação 1.5.2, por $M / \Gamma$, onde $M$ é uma variedade Riemanniana simplesmente conexa e completa e $\Gamma$ é um subgrupo discreto de isometrias de $(M, g)$ que age propriamente sobre $M$. Além disso, neste caso $\pi_{1}^{\text {orb }}(X)=$ $\Gamma$.

Definição 2.1.2 Seja $X=M / \Gamma$ um good orbifold Riemanniano. Definimos uma geodésica fechada de comprimento positivo como o par $\left(\omega,\left.\alpha\right|_{[0,1]}\right)$, onde $\alpha:[0,1] \rightarrow M$ é uma geodésica nãa constante e $\omega \in \Gamma$, e satisfazem que

$$
\omega \alpha(0)=\alpha(1) \quad e d \omega \dot{\alpha}(0)=\dot{\alpha}(1) .
$$

Observação 2.1.2 Sejam $X$ é um good orbifold e $\left(\omega,\left.\alpha\right|_{[0,1]}\right)$ uma geodésica fechada em $X$. Se $\tilde{\omega} \in \Gamma$, então $\left(\tilde{\omega} \omega \tilde{\omega}^{-1},\left.\tilde{\omega} \alpha\right|_{[0,1]}\right)$ também é uma geodésica fechada em X e $\operatorname{imagem}(\pi \circ \alpha)=$ imagem $(\pi \circ \tilde{\omega} \circ \alpha)$, onde $\pi: M \rightarrow M / \Gamma$ é a aplicação quociente respectiva. Mais ainda, se $\left(\omega_{1},\left.\alpha_{1}\right|_{[0,1]}\right)$ e $\left(\omega_{2},\left.\alpha_{2}\right|_{[0,1]}\right)$ são duas geodésicas fechadas em $X$ e se existe $\omega \in \Gamma$ tal que $\omega_{1}=\omega \omega_{2} \omega^{-1}$ e $\alpha_{1}=\omega \alpha_{2}$, então as duas geodésicas fechadas $\left(\omega_{1},\left.\alpha_{1}\right|_{[0,1]}\right)$ e $\left(\omega_{2},\left.\alpha_{2}\right|_{[0,1]}\right)$ representam a mesma geodésica em $X$, i.e., $\operatorname{imagem}\left(\pi \circ \alpha_{1}(\mathbb{R})\right)=\operatorname{imagem}\left(\pi \circ \alpha_{2}(\mathbb{R})\right)$. Agora, a recíproca não é verdadeira, i.e., se $\left(\omega_{1},\left.\alpha_{1}\right|_{[0,1]}\right)$ e $\left(\omega_{2},\left.\alpha_{2}\right|_{[0,1]}\right)$ são duas geodésicas fechadas em $X$ tais que imagem $\left(\pi \circ \alpha_{1}(\mathbb{R})\right)=\operatorname{imagem}\left(\pi \circ \alpha_{2}(\mathbb{R})\right)$, então isso não implica que exista um elemento $\omega \in \Gamma$ tal que $\omega_{1}=\omega \omega_{2} \omega^{-1}$. Vejamos o seguinte exemplo.

Exemplo 2.1.1 Seja $(M, g)=\left(\mathbb{S}^{2}, g_{0}\right)$ e $\Gamma$ o subgrupo de Iso $\left(\mathbb{S}^{2}, g_{\text {can }}\right)$ gerado pelas seguintes isometrias

$$
\begin{aligned}
& \omega_{1}: \mathbb{S}^{2} \quad \longrightarrow \quad \mathbb{S}^{2} \\
& (x, y, z) \longmapsto(x, y,-z) \\
& \omega_{2}: \mathbb{S}^{2} \quad \longrightarrow \quad \mathbb{S}^{2} \\
& (x, y, z) \longmapsto(-x,-y, z) .
\end{aligned}
$$

Assim $\Gamma=\left\{\omega_{1}, \omega_{2}, e,-e\right\}$, onde e é a isometria identidade de $\mathbb{S}^{2} e-e$ é a aplicação antípoda de $\mathbb{S}^{2}$. Sejam $\beta, \alpha:[0,1] \rightarrow \mathbb{S}^{2}$ as geodésicas definidas como $\alpha(t)=(\cos (2 \pi t), \operatorname{sen}(2 \pi t), 0)$ e $\beta(t)=(\cos (\pi t), \operatorname{sen}(\pi t), 0)$. Agora, temos que $\left(\omega_{1},\left.\alpha\right|_{[0,1]}\right),\left(e,\left.\alpha\right|_{[0,1]}\right) e\left(-e,\left.\beta\right|_{[0,1]}\right)$ são geodésicas fechadas em $X=\mathbb{S}^{2} / \Gamma$. Observemos que $\operatorname{imagem}(\pi \circ \alpha(\mathbb{R}))=\operatorname{imagem}(\pi \circ \beta(\mathbb{R}))$, mas $\omega_{1}$, e e-e não estão relacionados dois a dois por conjugação em $\Gamma$. 


\subsection{Ações Cocompactas e Função Deslocamento}

Definição 2.2.1 Sejam $(M, g)$ uma variedade Riemanniana e $\Gamma$ um subgrupo de Iso $(M, g)$. Dizemos que $\Gamma$ age cocompactamente sobre $M$ se existe um subconjunto compacto $K \subset M$ tal que $M=\bigcup_{\omega \in \Gamma} \omega(K)$.

Definição 2.2.2 Sejam $(M, g)$ uma variedade Riemanniana e $\omega$ um elemento de Iso $(M, g)$. Definimos a função deslocamento de $\omega$ da seguinte maneira

$$
\begin{aligned}
\delta_{\omega}: M & \longrightarrow[0, \infty) \\
x & \longmapsto d(x, \omega(x))
\end{aligned}
$$

Assim, também definimos $|\omega|:=\inf \left\{\delta_{\omega}(x): x \in M\right\}$ como o comprimento de translação de $\omega$. E o conjunto de pontos de $M$ que atingem este infimo é chamado o conjunto minimal de $\omega$ e será denotado por $\operatorname{Min}(\omega)$.

Definição 2.2.3 Sejam $(M, g)$ uma variedade Riemanniana e $\omega$ um elemento de Iso $(M, g) . \omega$ é chamado

1. Eliptico, se $\omega$ fixa algum ponto de $M$.

2. Hiperbólico, se Min $(\omega)$ é não vazio e $\delta_{\omega}$ é positivo.

3. Parabólico, se Min $(\omega)$ é vazio.

Se $\omega$ é uma isometria de tipo elíptico o de tipo hiperbólico, então dizemos que $\omega$ é semi-simples.

Proposição 2.2.1 Sejam $(M, g)$ uma variedade Riemanniana e $\Gamma$ um subgrupo de Iso $(M, g)$.

1. Se $\omega \in \operatorname{Iso}(M, g)$, então o conjunto Min $(\omega)$ é $\omega$-invariante.

2. Para cada $\omega \in \Gamma$ e para cada $\tilde{\omega} \in \operatorname{Iso}(M, g)$ temos que $\left|\tilde{\omega} \omega \tilde{\omega}^{-1}\right|=|\omega|$ e Min $\left(\tilde{\omega} \omega \tilde{\omega}^{-1}\right)=$ $\tilde{\omega}(\operatorname{Min}(\omega))$.

3. Se $\Gamma$ é discreto, age própria e cocompactamente sobre $M$, então cada elemento de $\Gamma$ é semisimples. Mais ainda, cada classe por conjugação de $\Gamma$ é semi-simples.

4. Se $\Gamma$ é discreto, age própria e cocompactamente sobre $M$, então $\{|\omega|: \omega \in \Gamma\}$ é um subconjunto discreto de $\mathbb{R}$.

5. Se $M$ é conexa e $\Gamma$ é discreto, age propriamente e cocompactamente sobre $M$, entẫo $\Gamma$ é finitamente gerado. 


\section{Demonstração.}

1. Segue-se da definição de $\operatorname{Min}(\omega)$.

2. Sejam $\omega \in \Gamma$ e $\tilde{\omega} \in \operatorname{Iso}(M, \mathrm{~g})$. Então para cada $x \in M$, temos que

$$
d\left(\tilde{\omega} \omega \tilde{\omega}^{-1}(x), x\right)=d\left(\omega \tilde{\omega}^{-1}(x), \tilde{\omega}^{-1}(x)\right)
$$

Assim, tomando o ínfimo sobre todos os elementos de $M$, temos que $\left|\tilde{\omega} \omega \tilde{\omega}^{-1}\right|=|\omega|$. A outra parte do item número 2 segue-se pela definição de $\operatorname{Min}\left(\tilde{\omega} \omega \tilde{\omega}^{-1}\right)$ e $\operatorname{Min}(\omega)$.

3. Supomos que $\Gamma$ age propriamente e cocompactamente sobre $M$. Logo, por definição, existe um subconjunto compacto $K \subset M$ tal que $M=\bigcup_{\omega \in \Gamma} \omega(K)$. Dado que $K$ é compacto, temos que o diámetro de $K$ é finito, assim, definimos $R:=\operatorname{diámetro}(K)$. Seja $\omega \in \Gamma$, então nosso objetivo é provar que $\operatorname{Min}(\omega)$ é um conjunto não vazio. Seja $\left\{x_{i}\right\}_{i \in \mathbb{N}}$ uma sequência em $M$ tal que $\lim _{j \rightarrow \infty} \delta_{\omega}\left(x_{n}\right)=|\omega|$. Agora, existe uma sequência $\left\{\omega_{i}\right\}_{i \in \mathbb{N}}$ em $\Gamma$ tal que $\omega_{i}\left(x_{i}\right) \in K$. Definamos $y_{i}:=\omega_{i}\left(x_{i}\right)$, então a sequência $\left\{y_{i}\right\}_{i \in \mathbb{N}}$ está contida em $K$.

Agora,

$$
\begin{aligned}
\lim _{i \rightarrow \infty} d\left(\omega_{i} \omega \omega_{i}^{-1}\left(y_{i}\right), y_{i}\right) & =\lim _{i \rightarrow \infty} d\left(\omega_{i} \omega\left(x_{i}\right), y_{i}\right) \\
& =\lim _{i \rightarrow \infty} d\left(\omega\left(x_{i}\right), \omega_{i}^{-1}\left(y_{i}\right)\right) \\
& =\lim _{i \rightarrow \infty} d\left(\omega\left(x_{i}\right), x_{i}\right) \\
& =|\omega|
\end{aligned}
$$

Portanto, a sequência $\left\{d\left(\omega_{i} \omega \omega_{i}^{-1}\left(y_{i}\right), y_{i}\right)\right\}_{i \in \mathbb{N}}$ é limitada, i.e., existe $L>0$ tal que

$$
d\left(\omega_{i} \omega \omega_{i}^{-1}\left(y_{i}\right), y_{i}\right) \leq L, \text { para todo } i \in \mathbb{N} .
$$

Agora sejam $x \in K$ e $i \in \mathbb{N}$, então

$$
\begin{aligned}
d\left(\omega_{i} \omega \omega_{i}^{-1}(x), x\right) & \leq d\left(\omega_{i} \omega \omega_{i}^{-1}(x), \omega_{i} \omega \omega_{i}^{-1}\left(y_{i}\right)\right)+d\left(\omega_{i} \omega \omega_{i}^{-1}\left(y_{i}\right), y_{i}\right)+d\left(y_{i}, x\right) \\
& \leq 2 d\left(y_{i}, x\right)+L \\
& \leq 2 R+L .
\end{aligned}
$$

Portanto, para cada $i \in \mathbb{N}$, temos que $\overline{B_{2 R+L}(x)} \cap \omega_{i} \omega \omega_{i}^{-1}\left(\overline{B_{2 R+L}(x)}\right) \neq \varnothing$. Agora, dado que $M$ é uma variedade Riemanniana completa, temos que $\overline{B_{2 R+L}(x)}$ é compacta. Então, dado que $\Gamma$ age descontinuamente sobre $M$, temos que $\left\{\omega_{i} \omega \omega_{i}^{-1}\right\}_{i \in \mathbb{N}}$ é finita. Assim $\left\{\omega_{i} \omega \omega_{i}^{-1}\right\}_{i \in \mathbb{N}}$ possui uma subsequência que é constante, e supomos que tal subsequência é $\left\{\omega_{i_{j}} \omega \omega_{i_{j}}^{-1}\right\}_{j \in \mathbb{N}}=\left\{\omega_{0}\right\}$, 
pois $\Gamma$ é discreto.

Por outro lado, dado que $K$ é compacto, temos que existe uma subsequência $\left\{y_{i_{j}}\right\}_{j \in \mathbb{N}}$ que converge a um $y_{0} \in K$.

Agora,

$$
\begin{aligned}
\delta_{\omega}\left(\omega_{i_{j}}^{-1}\left(y_{0}\right)\right) & =d\left(\omega \omega_{i_{j}}^{-1}\left(y_{0}\right), \omega_{i_{j}}^{-1}\left(y_{0}\right)\right)=d\left(\omega_{i_{j}} \omega \omega_{i_{j}}^{-1}\left(y_{0}\right), y_{0}\right)=d\left(\omega_{0}\left(y_{0}\right), y_{0}\right) \\
& =\lim _{j \rightarrow \infty} d\left(\omega_{0}\left(y_{i_{j}}\right), y_{i_{j}}\right)=\lim _{j \rightarrow \infty} d\left(\omega_{0} \omega_{i_{j}}\left(x_{i_{j}}\right), \omega_{i_{j}}\left(x_{i_{j}}\right)\right) \\
& =\lim _{j \rightarrow \infty} d\left(\omega_{i_{j}} \omega \omega_{i_{j}}^{-1} \omega_{i_{j}}\left(x_{i_{j}}\right), \omega_{i_{j}}\left(x_{i_{j}}\right)\right)=\lim _{j \rightarrow \infty} d\left(\omega\left(x_{i_{j}}\right), x_{i_{j}}\right) \\
& =|\omega| .
\end{aligned}
$$

Portanto Min $(\omega)$ é não vazio, e assim temos que $\omega$ é semi-simples.

Que as classes por conjugação de $\Gamma$ são semi-simples segue-se do item número 2 e do fato que cada elemento de que $\Gamma$ é semi-simples.

4. Supomos que $\Gamma$ age cocompactamente sobre $M$, i.e., existe $K \subset M$ compacto tal que $M=$ $\bigcup \omega(K)$. Vamos supor que $\{|\omega|: \omega \in \Gamma\}$ não é discreto, então existem uma sequência $\left\{\omega_{i}\right\}_{i \in \mathbb{N}}$ em $\Gamma$ e $s \in \mathbb{R}$ tais que $\lim _{i \rightarrow \infty}\left|\omega_{i}\right|=s$ e $\omega_{i} \neq \omega_{j}$ para todo par $i, j$ em $\mathbb{N}$, com $i \neq j$. Então, em especial, temos que $\omega_{i} \neq \omega_{j}$, para todo par $i, j$ em $\mathbb{N}$, com $i \neq j$. Pelo item número 3 temos que para cada $i \in \mathbb{N}$, o conjunto Min $\left(\omega_{i}\right)$ é não vazio, então seja $\left\{x_{i}\right\}_{i \mathbb{N}}$ uma sequência em $M$ tal que $x_{i} \in \operatorname{Min}\left(\omega_{i}\right)$. Agora, para cada $i \in \mathbb{N}$, existe $\bar{\omega}_{i}$ tal que $\bar{\omega}_{i}\left(x_{i}\right) \in K$. Logo, pelo item número 2 , temos que $\bar{\omega}_{i}\left(x_{i}\right) \in \operatorname{Min}\left(\bar{\omega}_{i} \omega \bar{\omega}_{i}^{-1}\right)$ e $|\omega|=\left|\bar{\omega}_{i} \omega \bar{\omega}_{i}^{-1}\right|$, logo podemos supor que cada $x_{i}$ está em $K$. Por outro lado, dado $x \in K, N \in \mathbb{N}$ tal que $d\left(\omega_{i}\left(x_{i}\right), x_{i}\right) \leq s+\frac{1}{2}$, para todo $i \geq N$. Assim, para $i \geq N$ temos que

$$
\begin{aligned}
d\left(\omega_{i}\left(x_{i}\right), x_{i}\right) & \leq d\left(\omega_{i}\left(x_{i}\right), x\right)+d\left(x_{i}, x\right) \\
& \leq s+\frac{1}{2}+r .
\end{aligned}
$$

Logo $\omega_{i}\left(B_{s+1 r}(x)\right) \cap B_{r}(x) \neq \varnothing$, para cada $i \geq N$, mas isso contradiz o fato que $\Gamma$ age propriamente sobre $M$. Então $\{|\omega|: \omega \in \Gamma\}$ é discreto.

5. Dado que $\Gamma$ age cocompactamente sobre $M$, então existe um subconjunto compacto $K \subset M$ tal que $M=\bigcup_{\omega \in \Gamma} \omega(K)$. Assim, dado $x \in K$, existe $r>0$ tal que $K \subset B_{r}(x)$. Portanto

$$
M=\bigcup_{\omega \in \Gamma} \omega\left(B_{r}(x)\right) .
$$

Seja $\mathcal{T}:=\left\{\omega \in \Gamma: \omega\left(B_{r}(x)\right) \cap B_{r}(x) \neq \varnothing\right\}$. Então temos que $\mathcal{T}$ é finito pois $\Gamma$ age 
descontinuamente sobre $M$. Observemos que $\mathcal{T}=\mathcal{T}^{-1}$. Assim, definamos por $\tilde{\Gamma}$ o subgrupo gerado por $\mathcal{T}$. Sejam

$$
U_{1}:=\bigcup_{\tilde{\omega} \in \tilde{\Gamma}} \tilde{\omega}\left(B_{r}(x)\right) \text { e } U_{2}:=\bigcup_{\omega \in \Gamma \backslash \tilde{\Gamma}} \omega\left(B_{r}(x)\right)
$$

Se $U_{1} \cap U_{2} \neq \varnothing$, então existem $\tilde{\omega} \in \tilde{\Gamma}$ e $\omega \in \Gamma \backslash \tilde{\Gamma}$ tais que $\omega^{-1} \tilde{\omega}\left(B_{r}(x)\right) \cap B_{r}(x) \neq \varnothing$. Então $\omega^{-1}$ é um elemento de $\tilde{\Gamma}$, que é um absurdo. Portanto $U_{1} \cap U_{2}=\varnothing$. Mas, dado que $M$ é conexa, temos que $U_{1} 0,=M, U_{2}=\varnothing$ e $\tilde{\Gamma}=\Gamma$.

Observação 2.2.1 Suponhamos que $X=M / \Gamma$ é um good orbifold Riemanniano onde $(M, g)$ é uma variedade Riemaniana completa e simplesmente conexa. Então, pela Proposição 3.5.1. temos que $\Gamma$ age cocompactamente se e somente se $X$ é compacto. Assim podemos dar a seguinte proposição.

Proposição 2.2.2 Suponhamos que $X=M / \Gamma$ é um good orbifold Riemanniano onde $(M, g)$ é uma variedade Riemaniana completa e $\Gamma$ age cocompactamente sobre $M$. Então existe uma cobertura de $M$ por bolas totalmente normais. Além disso, tal cobertura admite um número de Lebesgue.

Demonstração. Dado que $\Gamma$ age cocompactamente sobre $M$, temos que existe $K \subset M$ compacto e conexo tal que $M=\bigcup_{\omega \in \Gamma} \omega(K)$. Agora, existe uma cobertura de $K$ por bolas totalmente normais centradas em pontos de $K$, i.e.,

$$
K \subset\left\{B_{r_{x}}(x): x \in K\right\}
$$

onde cada $B_{r_{x}}(x)$ é uma bola totalmente normal. Mais ainda

$$
K \subset\left\{B_{r_{x} / 2}(x): x \in K\right\}
$$

Agora, dado que $K$ é compacto, podemos reduzir esta última cobertura a uma cobertura finita, i.e.,

$$
K \subset \bigcup_{1 \leq i \leq l} B_{r_{i} / 2}\left(x_{i}\right)
$$

onde cada $x_{i}$ está em $K$.

Definindo

$$
\mathcal{C}:=\bigcup_{\omega \in \Gamma} \bigcup_{1 \leq i \leq l} \omega\left(B_{r_{i}}\left(x_{i}\right)\right)
$$


temos que $\mathcal{C}$ é uma cobertura de $M$ por bolas totalmente normais. Agora seja

$$
\rho:=\min \left\{r_{i} / 2: i=1,2, \ldots l\right\} .
$$

Então afirmamos que $\rho$ é um número de Lebesgue para a cobertura $\mathcal{C}$. Com efeito, seja $A \subset M$ tal que o diámetro de $A$ é menor que $\rho$, e seja $y \in A$. Então existe um índice $i \in\{1,2, \ldots l\}$ e um $\omega \in \Gamma$ tais que $y \in B_{r_{i} / 2}\left(\omega\left(x_{i}\right)\right)$. Agora, seja $x \in A$, então

$$
\begin{aligned}
d\left(x, \omega\left(x_{i}\right)\right) & \leq d(x, y)+d\left(y, \omega\left(x_{i}\right)\right) \\
& <\rho+r_{i} / 2 \\
& \leq r_{i} .
\end{aligned}
$$

Portanto $A \subset B_{r_{i} / 2}\left(\omega\left(x_{i}\right)\right)$. Assim, dado que $A$ é qualquer subconjunto de $M$, temos que $\rho$ é um número de Lebesgue para a cobertura $\mathcal{C}$.

\subsection{Processo de Duplo Encurtamento}

Supomos que $X=M / \Gamma$ é um good orbifold Riemanniano compacto, e sejam $\alpha:[0,1] \rightarrow M$ uma curva suave por partes e $\omega$ um elemento de $\Gamma$ tais que

$$
2 E(\alpha)<\kappa \text { e } \omega(\alpha(0))=\alpha(1) \text {, onde } \kappa \text { é uma constante real positiva. }
$$

Observe que então

$$
\mathcal{L}\left(\left.\alpha\right|_{[0,1]}\right)=\int_{0}^{1}|\dot{\alpha}(t)| d t<\kappa
$$

Agora, pela Observação 2.2.1 e pela Proposição 2.2.2, temos que $M$ possui um número de Lebesgue $\rho$ para uma cobertura por bolas totalmente normais.

Por outra parte, dado que $\mathcal{L}\left(\left.\alpha\right|_{[0,1]}\right)<\kappa$, então existe uma partição $\left\{0=t_{0}<t_{1}<\cdots<t_{k-1}<\right.$ $\left.t_{k}=1\right\}$ do intervalo $[0,1]$ tal que $\left(t_{j}-t_{j-1}\right) \leq \frac{\rho^{2}}{\kappa}$, para todo $1 \leq j \leq k$. Agora, definindo $\alpha_{j}(t):=$ 
$\left.\alpha(t)\right|_{\left[t_{j-1}, t_{j}\right]}$, para $1 \leq j \leq k$, temos que

$$
\begin{aligned}
\mathcal{L}\left(\alpha_{j}\right) & =\int_{t_{j-1}}^{t_{j}}\left|\dot{\alpha}_{j}(t)\right| d t \\
& =\left[\int_{t_{j-1}}^{t_{j}}\left|\dot{\alpha}_{j}(t)\right|^{2} d t\right]^{1 / 2}\left[t_{j}-t_{j-1}\right]^{1 / 2} \\
& =\left[2 E\left(\alpha_{j}\right)\right]^{1 / 2}\left[t_{j}-t_{j-1}\right]^{1 / 2} \\
& \leq[2 E(\alpha)]^{1 / 2}\left[t_{j}-t_{j-1}\right]^{1 / 2} \\
& <\rho_{0}
\end{aligned}
$$

Então, dado que $\rho$ é um número de Lebesque para uma cobertura de $M$ por bolas totalmente normais, temos que cada $\left.\alpha\right|_{\left[t_{j-1}, t_{j}\right]}$ está contida numa bola totalmente normal. Assim, para cada $1 \leq j \leq k$, existe um único segmento minimizante $\gamma_{j}:\left[t_{j-1}, t_{j}\right] \rightarrow M$, ligando $\alpha\left(t_{j-1}\right)$ e $\alpha\left(t_{j}\right)$.

Definamos $\alpha_{0}:=\gamma_{1} * \gamma_{2} * \cdots * \gamma_{k}$, i.e., $\alpha_{0}$ é a concatenação dos segmentos $\gamma_{j}:\left[t_{j-1}, t_{j}\right] \rightarrow M$, para $j=1,2, \ldots, k-1, k$, então $\alpha_{0}:[0,1] \rightarrow M$ é uma geodésica minimizante por partes. Além disso, temos o seguinte

$$
2 E\left(\alpha_{0}\right)=2 \sum_{j=1}^{k} E\left(\gamma_{j}\right) \leq 2 \sum_{j=1}^{k} E\left(\alpha_{j}\right)=2 E(\alpha)<\kappa \text { e } \omega\left(\alpha_{0}(0)\right)=\alpha_{0}(1) .
$$

Assim, damos a seguinte definição.

Definição 2.3.1 Definimos $\alpha_{0}$ como o encurtamento de $\alpha$ com nodos

$$
\left\{0=t_{0}, t_{1}, \ldots, t_{k-1}, t_{k}=1\right\}
$$

Observemos que a curva $\alpha_{0}:[0,1] \rightarrow M$ satisfaz as mesmas condições da curva $\alpha:[0,1] \rightarrow M$. E, dado que $\left\{0=t_{0}<t_{1}<\cdots<t_{k-1}<t_{k}=1\right\}$ é uma partição do intervalo [0,1], tal que $\left(t_{j}-t_{j-1}\right) \leq \frac{\rho^{2}}{\kappa}$, para todo $1 \leq j \leq k$, então existe uma família $\left\{\tau_{1}, \tau_{2}, \ldots, \tau_{k-1}, \tau_{k}\right\}$ tal que

$$
t_{0}=0<\tau_{1}<t_{1}<\cdots<\tau_{k-1}<t_{k-1}<\tau_{k}<t_{n}=1 \text { e }\left(\tau_{j}-\tau_{j-1}\right) \leq \frac{\rho^{2}}{\kappa}, \quad \text { para todo } 2 \leq j \leq k .
$$


Assim, seja $\tau_{0}:=1-\tau_{k}$ e definamos

$$
\tilde{\alpha}_{0}(t):=\left\{\begin{array}{lll}
\omega^{-1} \alpha_{0}(t+1) & \text { se } & t \in\left[\tau_{0}, 0\right] ; \\
\alpha_{0}(t) & \text { se } & t \in[0,1] .
\end{array}\right.
$$

Logo $\tilde{\alpha}_{0}:\left[\tau_{0}, 1\right] \rightarrow M$ é uma geodésica minimizante por partes e uma extensão de $\alpha_{0}$.

Agora,

$$
\begin{aligned}
2 E\left(\left.\tilde{\alpha}_{0}\right|_{\left[\tau_{0}, \tau_{k}\right]}\right) & =2 E\left(\left.\tilde{\alpha}_{0}\right|_{\left[\tau_{0}, 0\right]}\right)+2 E\left(\left.\tilde{\alpha}_{0}\right|_{\left[0, \tau_{k}\right]}\right) \\
& =2 E\left(\left.\alpha_{0}\right|_{\left[\tau_{k}, 1\right]}\right)+2 E\left(\left.\alpha_{0}\right|_{\left[0, \tau_{k}\right]}\right) \\
& =2 E\left(\left.\alpha_{0}\right|_{[0,1]}\right) \\
& =2 E(\alpha) \\
& <\kappa .
\end{aligned}
$$

Assim, fazendo o processo de encurtamento para $\left.\tilde{\alpha}_{0}\right|_{\left[\tau_{0}, \tau_{k}\right]} \operatorname{com} \operatorname{nodos}\left\{\tau_{0}, \tau_{1}, \tau_{2}, \ldots, \tau_{k-1}, \tau_{k}\right\}$, obtemos uma curva $\tilde{\alpha}_{0}^{*}:=\beta_{1} * \beta_{2} * \cdots * \beta_{k}$, onde $\beta_{j}:\left[\tau_{j-1}, \tau_{j}\right] \rightarrow M$ é o único segmento de geodésica minimizante ligando $\hat{\alpha}_{0}\left(\tau_{j-1}\right)$ e $\hat{\alpha}_{0}\left(\tau_{j}\right)$, para $1 \leq j \leq k$, respectivamente. Então $\hat{\alpha}_{0}^{*}:\left[\tau_{0}, \tau_{k}\right] \rightarrow M$ é uma geodésica por partes, $E\left(\tilde{\alpha}_{0}^{*}\right) \leq E\left(\left.\alpha_{0}\right|_{\left[\tau_{0}, \tau_{k}\right]}\right)$ e $\omega\left(\hat{\alpha}_{0}^{*}\left(\tau_{0}\right)\right)=\tilde{\alpha}_{0}^{*}\left(\tau_{k}\right)$.

Definamos

$$
P(\alpha)(t):= \begin{cases}\omega \alpha_{0}^{*}(t-1) & \text { se } t \in\left[\tau_{k}, 1\right] \\ \alpha_{0}^{*}(t) & \text { se } t \in\left[\tau_{0}, \tau_{k}\right] .\end{cases}
$$

Então $P(\alpha)$ é uma extenção de $\alpha_{0}^{*}$ a todo o intervalo $\left[\tau_{0}, 1\right]$,

$$
\begin{aligned}
E\left(\left.P(\alpha)\right|_{[0,1]}\right) & =E\left(\left.P(\alpha)\right|_{\left[0, \tau_{k}\right]}\right)+E\left(\left.\tilde{\alpha}_{0}^{*}\right|_{\left[\tau_{0}, 0\right]}\right)=E\left(\tilde{\alpha}_{0}^{*}\right) \\
& \leq E\left(\left.\tilde{\alpha}_{0}\right|_{\left[\tau_{0}, \tau_{k}\right]}\right)=E\left(\left.\tilde{\alpha}_{0}\right|_{\left[\tau_{0}, 0\right]}\right)+E\left(\left.\tilde{\alpha}_{0}\right|_{\left[0, \tau_{k}\right]}\right) \\
& =E\left(\left.\alpha_{0}\right|_{[0,1]}\right)=E\left(\alpha_{0}\right) \\
& \leq E(\alpha)
\end{aligned}
$$

e $\omega(P(\alpha(0)))=P(\alpha(1))$. Assim, damos a seguinte definição.

Definição 2.3.2 $\left.P(\alpha)\right|_{[0,1]}$ é chamada duplo encurtamento de $\alpha$. 
A seguinte proposição será útil na Proposição 2.3.2 e no Teorema 2.3.1, e sua prova pode ser vista em [AJ11].

Proposição 2.3.1 Sejam $(M, g)$ uma variedade Riemanniana e $\Gamma$ um subgrupo de Iso $(M, g)$ tal que $M / \Gamma$ é um good orbifold compacto. Sejam $\alpha:[0,1] \rightarrow M$ uma curva em $M$ e $\omega \in \Gamma$ tais que $\omega(\alpha(0))=\alpha(1)$ e $2 E(\alpha) \leq \kappa$, onde $\kappa$ é uma constante positiva. Então $E\left(\left.P(\alpha)\right|_{[0,1]}\right) \leq E(\alpha)$, e a igualdade é verdadeira se e somente se $\left(\omega,\left.\alpha\right|_{[0,1]}\right)$ é uma geodésica fechada em $M / \Gamma$.

Proposição 2.3.2 Supomos que $(M, g)$ uma variedade Riemanniana e $\Gamma$ um subgrupo de Iso $(M, g)$ tal que $M / \Gamma$ é um good orbifold compacto. Sejam $\alpha:[0,1] \rightarrow M$ uma curva em $M$ e $\omega \in \Gamma$ tais que $\omega(\alpha(0))=\alpha(1)$ e $2 E(\alpha) \leq \kappa$, onde $\kappa$ é uma constante positiva. Então exite uma subsequência $\left\{\gamma_{m}:=P^{n_{m}}(\alpha)\right\}_{m \in \mathbb{N}}$ de $\left\{\gamma_{n}:=P^{n}(\alpha)\right\}_{n \in \mathbb{N}}$ e uma sequência $\left\{k_{m}\right\}_{m \in \mathbb{N}}$ de elementos em $\Gamma$ tais que $\left\{k_{m} \gamma_{m}\right\}_{m \in \mathbb{N}}$ converge para uma geodésica fechada $\left(\bar{\omega},\left.\gamma\right|_{[0,1]}\right)$. Noutras palavras, a subsequência de classes $\left\{P^{n_{m}}(\alpha)\right\}_{m \in \mathbb{N}}$ converge uniformemente a uma (possivelmente trivial) geodésica fechada de $M / \Gamma$.

Demonstração. Primeiro observemos que $\left(\omega, \gamma_{n}\right)$ é uma geodésica fechada por partes em $M / \Gamma$, com nodos $\gamma_{n}\left(\tau_{0}\right), \gamma_{n}\left(\tau_{1}\right), \ldots, \gamma_{n}\left(\tau_{k}\right)$. Logo temos uma sequência

$$
\left(\left\{\gamma_{n}\left(\tau_{0}\right)\right\}_{n \in \mathbb{N}}, \ldots,\left\{\gamma_{n}\left(\tau_{k}\right)\right\}_{n \in \mathbb{N}}\right)
$$

em $M^{k}$. Agora, dado que $M / \Gamma$ é compacto, podemos encontrar uma subsequência da sequência $\left\{\gamma_{n}:=P^{n}(\alpha)\right\}_{n \in \mathbb{N}}$ (que chamaremos da mesma forma) com nodos $\left(\left\{\gamma_{n}\left(\tau_{0}\right)\right\}_{n \in \mathbb{N}}, \ldots,\left\{\gamma_{n}\left(\tau_{k}\right)\right\}_{n \in \mathbb{N}}\right)$, uma sequência $\left\{k_{n}\right\}_{n \in \mathbb{N}}$ de elementos em $\Gamma$ e pontos $p_{0}, p_{1}, \ldots, p_{k} \in M$, tais que $k_{n} \gamma_{n}\left(\tau_{j}\right) \rightarrow p_{j}$, para cada $j=0,1, \ldots, k$.

Definindo $\omega_{n}:=k_{n} \omega k_{n}^{-1}$, obtemos que $\omega_{n} k_{n} \gamma_{n}(t)=k_{n} \omega \gamma_{n}(t)$, e assim

$$
\omega_{n} k_{n} \gamma_{n}(t)=k_{n} \omega \gamma_{n}(t)=k_{n} \gamma_{n}(t+1) \text {, para todo } t \in\left[\tau_{0}, 0\right]
$$

$\operatorname{Logo}\left(\omega_{n},\left.k_{n} \gamma_{n}\right|_{\left[\tau_{0}, 1\right]}\right)$ é uma geodésica fechada por partes de $M / \Gamma$.

Agora

$$
k_{n} \gamma_{n}\left(\tau_{0}\right) \rightarrow p_{0} \text { e } \omega_{n} k_{n} \gamma_{n}\left(\tau_{0}\right)=k_{n} \omega \gamma_{n}\left(\tau_{0}\right)=k_{n} \gamma_{n}\left(\tau_{k}\right) \rightarrow p_{k}
$$

Então, dado que $\Gamma$ age propriamente sobre $M$, existe uma subsequência de $\left\{\omega_{n}\right\}_{n \in \mathbb{N}}$ (que chamaremos da mesma forma), que converge a um $\bar{\omega} \in \Gamma$. Como $\Gamma$ é discreto, então existe $N \in \mathbb{N}$, tal que $\omega_{n}=\bar{\omega}$, para todo $n \geq N$. Agora, pela continuidade da aplicaçãoo exponencial, temos que a sequência de 
curvas $\left\{k_{n} \gamma_{n}\right\}_{n \in \mathbb{N}}$ converge uniformemente para uma geodésica por partes $\gamma_{0}$ em $M$ com nodos $\gamma_{0}\left(\tau_{0}\right)=p_{0}, \ldots, \gamma_{0}\left(\tau_{k}\right)=p_{k}$.

Por outro lado

$$
\bar{\omega} \gamma_{0}(t)=\lim _{n \rightarrow \infty} \bar{\omega} k_{n} \gamma_{n}(t)=\lim _{n \rightarrow \infty} \bar{\omega}_{n} k_{n} \gamma_{n}(t), \text { para todo } t \in\left[\tau_{0}, 1\right]
$$

Em particular, se $t \in\left[\tau_{0}, 0\right]$, temos que

$$
\bar{\omega} \gamma_{0}(t)=\lim _{n \rightarrow \infty} \omega_{n} k_{n} \gamma_{n}(t)=\lim _{n \rightarrow \infty} k_{n} \gamma_{n}(t+1)=\gamma_{0}(t+1)
$$

Portanto $\left(\bar{\omega},\left.\gamma_{0}\right|_{\left[\tau_{0}, 1\right]}\right)$ é uma geodésica fechada por partes de $M / \Gamma$.

Definamos $\mu(m) \geq 1$, de tal maneira que $\gamma_{m+1}=P^{\mu(m)}\left(\gamma_{m}\right)$. Agora

$$
E\left(\gamma_{m}\right)=\sum_{i=1}^{k} E\left(\gamma_{m}^{i}\right)=\sum_{i=1}^{k} \frac{\left[l\left(\gamma_{m}^{i}\right)\right]^{2}}{2\left(\tau_{i}-\tau_{i-1}\right)}
$$

onde $\gamma_{m}^{i}:=\left.\gamma_{m}\right|_{\left[\tau_{i-1}, \tau_{i}\right]}$. Sabemos que $E\left(k_{m} \gamma_{m}\right)=E\left(\gamma_{m}\right)$, assim temos que

$$
\lim _{m \rightarrow \infty} E\left(\gamma_{m}\right)=\lim _{m \rightarrow \infty} E\left(k_{m} \gamma_{m}\right)=\sum_{i=1}^{k} \lim _{m \rightarrow \infty} \frac{\left[l\left(k_{m} \gamma_{m}^{i}\right)\right]^{2}}{2\left(\tau_{i}-\tau_{i-1}\right)}=\sum_{i=1}^{k} \frac{\left[l\left(\gamma_{0}^{i}\right)\right]^{2}}{2\left(\tau_{i}-\tau_{i-1}\right)}=E\left(\gamma_{0}\right)
$$

Agora

$$
E\left(\gamma_{0}\right)=\lim _{m \rightarrow \infty} E\left(\gamma_{m+1}\right)=\lim _{m \rightarrow \infty} E\left(P^{\mu(m)}\left(\gamma_{m}\right)\right) \leq \lim _{m \rightarrow \infty} E\left(P\left(\gamma_{m}\right)\right) \leq \lim _{m \rightarrow \infty} E\left(\gamma_{m}\right)=E\left(\gamma_{0}\right) .
$$

Portanto $E\left(\gamma_{0}\right)=\lim _{m \rightarrow \infty} E\left(P\left(\gamma_{m}\right)\right)$.

Agora afirmamos que

$$
E\left(P\left(\gamma_{m}\right)\right)=E\left(P\left(k_{m} \gamma_{m}\right)\right) \rightarrow E\left(P\left(\gamma_{0}\right)\right) .
$$

Logo

$$
\lim _{m \rightarrow \infty} E\left(P\left(k_{m} \gamma_{m}\right)\right) \rightarrow E\left(\gamma_{0}\right)
$$

e

$$
\lim _{m \rightarrow \infty} E\left(P\left(k_{m} \gamma_{m}\right)\right) \rightarrow E\left(P\left(\gamma_{0}\right)\right)
$$

Então $E\left(\gamma_{0}\right)=E\left(P\left(\gamma_{0}\right)\right)$. Assim, pela Proposição 2.3.1, temos que $\left(\bar{\omega},\left.\gamma_{0}\right|_{[0,1]}\right)$ é uma geodésica fechada em $M / \Gamma$. 
Teorema 2.3.1 Sejam $M$ uma variedade Riemanniana conexa completa e $\Gamma$ um subgrupo infinito discreto de isometrias de $M$ tal que $\Gamma$ age propriamente sobre $M$ e $M / \Gamma$ é um good orbifold compacto. Vamos supor que alguma das seguintes condiçôes é satisfeita:

1. $\Gamma$ admite infinitas classes por conjugação (e.g., Г é abeliano);

2. existe um elemento $\omega \in \Gamma$ sem pontos fixos (e.g., $\omega$ tem ordem infinita).

Então existe uma geodésica fechada não trivial em $M / \Gamma$. Em particular, se a condição (2) é satisfeita, então para cada curva a ligando $x$ e $\omega(x)$, existe uma subsequência de classes de interaçôes de duplo encurtamento de a que converge para uma geodésica fechada no trivial em $M / \Gamma$.

Demonstração. Primeiro vamos supor que $\Gamma$ admite infinitas classes por conjugação. Então seja $\left\{\omega^{i}\right\}_{i \in \mathbb{N}} \subset \Gamma$ uma sequência tal que $\omega^{i} \nsim \omega^{j}$ (i.e., $\omega^{i}$ e $\omega^{j}$ não estão relacionados por conjugação) se $i \neq j$. Seja $x$ um ponto em $M$. Como $\Gamma_{x}$ é finito, então existe uma subsequência de $\left\{\omega^{i}\right\}_{i \in \mathbb{N}}$ (que chamaremos da mesma forma) tal que $\omega^{i}(x) \neq x$, para cada $i \in \mathbb{N}$.

Para cada $i \in \mathbb{N}$, seja $\alpha^{i}:[0,1] \rightarrow M$ uma curva ligando $x$ e $\omega^{i}(x)$. Definamos $x_{n}^{i}:=P^{n}\left(\alpha^{i}\right)(0)$, onde $P^{n}\left(\alpha^{i}\right)$ é a n-ésima iteração do processo de duplo encurtamento de $\alpha$. Agora, $\omega^{i} P^{n}\left(\alpha^{i}\right)(0)=$ $\omega^{i}\left(x_{n}^{i}\right)$. Pela Proposição 2.3.2 existe uma sequência $\left\{k_{n}^{i}\right\}_{n \in \mathbb{N}}$ em $\Gamma$ e uma subsequência de $\left\{\gamma_{n}^{i}:=\right.$ $\left.P^{n}\left(\alpha^{i}\right)\right\}_{n \in \mathbb{N}}$ (que chamaremos da mesma maneira), tais que $\left\{k_{n}^{i} \gamma_{n}^{i}\right\}_{n \in \mathbb{N}}$ converge para uma geodésica fechada em $M / \Gamma$, que pode ser trivial. Noutras palavras, $\left(\omega^{i},\left.k_{n}^{i} \gamma_{n}^{i}\right|_{[0,1]}\right)$ converge uniformemente para $\left(\bar{\omega}^{i},\left.\gamma^{i}\right|_{[0,1]}\right)$, onde $\left(\bar{\omega}^{i},\left.\gamma^{i}\right|_{[0,1]}\right)$ é uma geodésica fechada em $M / \Gamma$ (que pode ser trivial), onde $\omega_{n}^{i}=k_{n}^{i} \omega^{i}\left(k_{n}^{i}\right)^{-1}$ e $\bar{\omega}^{i}=\omega_{n}^{i}$, para todo $n \geq N$, para um $N \in \mathbb{N}$, fixado.

Definamos $x^{i}:=\gamma^{i}(0)$ e $y^{i}:=\gamma^{i}(1)$. Por definição $x_{n}^{i}=P^{n}\left(\alpha^{i}\right)(0)$, e sabemos que $k_{n}^{i} \gamma_{n}^{i} \rightarrow \gamma^{i}$, então

$$
k_{n}^{i}\left(x_{n}^{i}\right) \rightarrow \gamma^{i}(0)=x^{i} \text { e } k_{n}^{i} \omega^{i}\left(x_{n}^{i}\right)=k_{n}^{i} \gamma_{n}^{i}(1) \rightarrow \gamma^{i}(1)=y^{i}
$$

Vamos supor que para cada $i \in \mathbb{N}$, a curva $\gamma^{i}$ é trivial e vamos obter um absurdo. Assim, dado que estamos supondo que cada $\gamma^{i}$ é trivial, temos que $x^{i}=y^{i}$, para cada $i \in \mathbb{N}$.

Agora defimamos $g_{n}^{i}:=k_{n}^{i}\left(\omega^{i}\right)^{-1}\left(k_{n}^{i}\right)^{-1}$. Então, por (2.2), temos que

$$
g_{n}^{i}\left(k_{n}^{i} \omega^{i}\left(x_{n}^{i}\right)\right)=k_{n}^{i}\left(x_{n}^{i}\right) \rightarrow x^{i} \text { e } k_{n}^{i} \omega^{i}\left(x_{n}^{i}\right) \rightarrow y^{i}=x^{i}
$$

Como $\Gamma$ age propriamente em $M$, então existe uma subsequência de $\left\{g_{n}^{i}\right\}_{n \in \mathbb{N}}$ (que chamaremos da mesma forma) que é convergente, i.e., $g_{n}^{i} \rightarrow g^{i}$, onde $g^{i} \in \Gamma$ e $g^{i} \in \Gamma_{x_{i}}$. Além disso, dado que $\Gamma$ é discreto, temos que existe $n_{0}$ tal que $g_{n}^{i}=g^{i}$, para cada $n \geq n_{0}$. Portanto $g_{n}^{i} \in \Gamma_{x^{i}}$ se $n \geq n_{0}$. 
Supomos que $n \geq n_{0}$, logo

$$
\left(k_{n}^{i}\right)^{-1}\left(g^{i}\right)^{-1} k_{n}^{i}=\left(k_{n}^{i}\right)^{-1}\left(g_{n}^{i}\right)^{-1} k_{n}^{i}=\left(k_{n}^{i}\right)^{-1}\left(k_{n}^{i} \omega^{i}\left(k_{n}^{i}\right)^{-1}\right) k_{n}^{i}=\omega^{i} .
$$

E, dado que $g^{i} \in \Gamma_{x^{i}}$, temos

$$
\left(k_{n}^{i}\right)^{-1}\left(g^{i}\right)^{-1} k_{n}^{i}\left(\left(k_{n}^{i}\right)^{-1}\left(x^{i}\right)=\left(k_{n}^{i}\right)^{-1}\left(g^{i}\right)^{-1}\left(x^{i}\right)=\left(k_{n}^{i}\right)^{-1}\left(x^{i}\right) .\right.
$$

Logo $\omega^{i}\left(\left(k_{n}^{i}\right)^{-1}\left(x^{i}\right)=\left(k_{n}^{i}\right)^{-1}\left(x^{i}\right)\right.$, e portanto $\omega^{i} \in \Gamma_{\left(k_{n}^{i}\right)^{-1}\left(x^{i}\right)}$.

Pela compacidade de $M / \Gamma$, existem uma subsequência de $\left\{\left(k_{n}^{i}\right)^{-1} x^{i}\right\}_{i \in \mathbb{N}}$ (que chamaremos da mesma forma), uma sequência $\left\{h^{i}\right\}_{i \in \mathbb{N}} \subset \Gamma$ e $\bar{x} \in M$, tais que $h^{i}\left(k_{n}^{i}\right)^{-1}\left(x^{i}\right) \rightarrow \bar{x}$. Assim, sejam $i \geq i_{0}$, para um $i_{0} \in \mathbb{N}$, suficientemente grande, e $n \geq n_{0}$. Então, como $\omega^{i} \in \Gamma_{\left(k_{n}^{i}\right)^{-1}\left(x^{i}\right)}$, temos que a família $h^{i} \omega^{i}\left(h_{n}^{i}\right)^{-1} \in \Gamma_{h^{i}\left(k_{n}^{i}\right)^{-1}\left(x^{i}\right)}=\Gamma_{\bar{x}}$. Dado que $\Gamma_{\bar{x}}$ é finito, temos que $\left\{h^{i} \omega^{i}\left(h^{i}\right)^{-1}\right\}_{i \in \mathbb{N}}$ é finito, que é uma contradição, pois a família $\left\{\omega^{i}\right\}_{i \in \mathbb{N}}$ é tal que todos seus elementos não estão relacionados por conjugação. Portanto existe um $i_{1}$ tal que $\left(\omega^{i_{1}},\left.\gamma^{i_{1}}\right|_{[0,1]}\right)$ é uma geodésica fechada em $M / \Gamma$ e é não trivial. E assim temos o resultado quando temos a hipótese do item número 1.

Vamos supor que existe $\omega \in \Gamma$ que não possui pontos fixos. Sejam $x \in M$ e $\alpha:[0,1] \rightarrow M$ uma curva suave, tais que $\alpha$ liga $x$ e $\omega(x)$. Seja $P^{n}\left(\alpha^{i}\right)$ a n-ésima iteração do processo de duplo encurtamento de $\alpha$. Agora, pela Proposição 2.3.2, existe uma sequência $\left\{k_{n}^{i}\right\}_{n \in \mathbb{N}}$ em $\Gamma$ e uma subsequência de $\left\{\gamma_{n}:=P^{n}(\alpha)\right\}_{n \in \mathbb{N}}$ (que chamaremos da mesma maneira), tais que $\left\{k_{n} \gamma_{n}\right\}_{n \in \mathbb{N}}$ converge para uma geodésica fechada em $M / \Gamma$, i.e., $\left(k_{n} \omega k_{n}^{-1},\left.k_{n} \gamma_{n}\right|_{[0,1]}\right)$ converge uniformemente para $\left(\bar{\omega},\left.\gamma\right|_{[0,1]}\right)$, onde $\left(\bar{\omega},\left.\gamma\right|_{[0,1]}\right)$ é uma geodésica fechada em $M / \Gamma$. Seja $x_{0}=\gamma(0)$, e vamos supor que $\left(\bar{\omega},\left.\gamma\right|_{[0,1]}\right)$ é trivial, então vamos obter que $\omega \in \Gamma_{\left(k_{n}\right)^{-1}\left(x_{0}\right)}$, para $n \geq n_{0}$, com $n_{0}$ suficientemente grande (vide prova do item número 1). Mas isto é um absurdo, pois $\omega$ não fixa pontos de $M$. Logo vale o teorema si temos a hipótese do item n $\widetilde{A}^{\mathrm{o}}$ mero 2 .

O seguinte teorema mostra que se o espaço de órbitas de uma folheação Riemannina, com algumas hipóteses adicionais, e tal que é um orbifold Riemanniano, admite uma geodésica fechada não trivial. Para uma prova do teorema vide [AJ11].

Teorema 2.3.2 Sejam $(M, g)$ uma variedade Riemanniana completa e $\mathcal{F}$ uma folheação Riemanniana tal que as folhas de $\mathcal{F}$ são fechadas e mergulhadas. Suponhamos que $\pi_{1}(M)$ é finito e que $M / \mathcal{F}$ é uma orbifold Riemanniana compacta. Então $M / \mathcal{F}$ admite uma geodésica fechada não 
trivial.

\subsection{Existência de Geodésicas Fechadas num Orbifold de Curvatura Não-Negativa ou Não-Positiva}

Teorema 2.4.1 Seja $X=M / \Gamma$ um good orbifold Riemanniano compacto. Vamos supor que temos alguma das seguintes hipóteses

1. $\Gamma$ é finito;

2. existe um elemento em $\Gamma$ de ordem infinita.

Então X possui uma geodésica fechada não trivial.

Demonstração. Primeiro vamos supor que $\Gamma$ é finito. Neste caso vamos obter que então $M$ é uma variedade Riemanniana compacta. Portanto existe uma geodésica fechada não trivial $\alpha:[0,1] \rightarrow M$ em $M$. Então $\left(e,\left.\alpha\right|_{[0,1]}\right)$ é uma geodésica fechada e não trivial em $X$, onde $e$ é o elemento identidade de $\Gamma$.

Agora vamos supor que existe $\omega$ em $\Gamma$ de ordem infinita. Em particaular, $\omega$ não fixa pontos. Então, pelo item número 3 da Proposição 2.2.1, temos que $\omega$ é hiperbólico, i.e., Min $(\omega)$ é não vazio. Seja $x \in \operatorname{Min}(\omega)$. Agora, dado que $M$ é uma variedade Riemanniana completa, temos que existe uma geodésica minimizante $\alpha:[0,1] \rightarrow M$ ligando $x$ e $\omega(x)$, i.e., $\alpha(0)=x, \alpha(1)=$ $\omega(x)$ e $|\omega|=d(x, \omega(x))=\mathcal{L}\left(\left.\alpha\right|_{[0,1]}\right)$, onde $\mathcal{L}(\cdot)$ denota o comprimento de uma curva em $M$. Nosso objetivo é provar que $\left(\omega,\left.\alpha\right|_{[0,1]}\right)$ é uma geodésica fechada. i.e., $\omega(\alpha(0))=\alpha(1)$ e $d \omega(\dot{\alpha}(0))=\dot{\alpha}(1)$. Primeiramente observemos que $\left.\left.\alpha\right|_{[0,1]} * \omega \alpha\right|_{[0,1]}$, i.e., a concatenação de $\left.\alpha(t)\right|_{[0,1]}$ e $\omega\left(\left.\alpha(t)\right|_{[0,1]}\right)$, é uma geodésica por partes e $\alpha(1)$ é o único possível ponto onde a curva $\left(\left.\alpha(t)\right|_{[0,1]}\right) * \omega\left(\left.\alpha(t)\right|_{[0,1]}\right)$ não é diferenciável. Provar que $\left(\omega,\left.\alpha\right|_{[0,1]}\right)$ é uma geodésica fechada é equivalente a provar que $\left.\alpha(t)\right|_{[0,1]} * \omega\left(\left.\alpha(t)\right|_{[0,1]}\right)$ é uma geodésica suave, então vamos provar que $\left.\alpha(t)\right|_{[0,1]} * \omega\left(\left.\alpha(t)\right|_{[0,1]}\right)$ é diferenciável em $\alpha(1)$. Definindo $y:=\alpha(1 / 2)$, temos que

$$
\begin{aligned}
0<d(y, \omega(y)) & \leq d(y, \omega(x))+d(\omega(x), \omega(y)) \\
& \leq d(y, \omega(x))+d(x, y) \\
& =d(x, \omega(x)) \\
& =|\omega| .
\end{aligned}
$$


Então $y \in \operatorname{Min}(\omega)$ e $d(y, \omega(y))=d(y, \omega(x))+d(\omega(x), \omega(y))$. Assim temos que

$$
\left.\left.\alpha(t)\right|_{\left[\frac{1}{2}, 1\right]} * \omega \alpha(t)\right|_{\left[0, \frac{1}{2}\right]}
$$

é uma geodésica suave, e portanto $\left(\omega,\left.\alpha\right|_{[0,1]}\right)$ é uma geodésica fechada em $X$, como desejávamos provar.

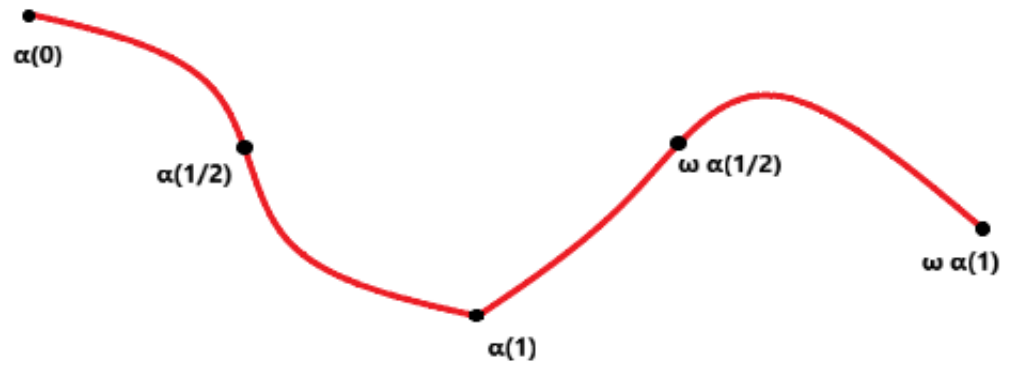

Figura 2.1: A curva $\left.\left.\alpha(t)\right|_{[0,1]} * \omega \alpha(t)\right|_{[0,1]}$.

Definição 2.4.1 Seja $X$ um orbifold Riemanniano. Dado $\bar{x} \in X$, definimos a curvatura seccional $k_{\bar{x}}$, como a curvatura seccional em um de seus pontos de levantamento. Mais ainda, dizemos que $X$ é de curvatura positiva (respectivamente não negativa, zero, negativa, não positiva) se a curvatura seccional de cada ponto de $X$ tem o respectivo sinal.

Antes de continuar, vamos dar um resultado que será fundamental no desenvolvimento dos seguintes resultados (vide [BH99], Corolário 2.16).

Proposição 2.4.1 (Gromov) Cada orbifold Riemanniano de curvatura seccional não-positiva é um good orbifold Riemanniano.

Proposição 2.4.2 Seja X um orbifold Riemanniano compacto.

1. Se X é um good orbifold de curvatura positiva, então $\pi_{1}^{\text {orb }}(X)$ é finito.

2. Se $X$ é de curvatura seccional negativa, então $X$ é um good orbifold e $\pi_{1}^{\text {orb }}(X)$ é infinito $e$ não é de torsão.

\section{Demonstração.}

1. Vamos supor que $X$ é um good orbifold Riemanniano compacto de curvatura positiva. Então podemos supor que $X=M / \Gamma$, onde $(M, \mathrm{~g})$ é uma variedade Riemanniana simplesmente co- 
nexa completa e $\Gamma$ é um subgrupo discreto de isometrias de Iso $(M, \mathrm{~g})$ que age propriamente sobre $M$. Agora, temos dois casos possíveis:

- existe $\epsilon>0$ tal que $k_{\bar{x}}>\epsilon$, para cada $\bar{x} \in X$, ou

- $k_{\bar{x}}>0$, para cada $\bar{x} \in X$.

Então vamos trabalhar em cada caso. No primeiro caso vamos ter que $k_{x}>\epsilon>0$, para cada $x \in M$. Logo, pelo Teorema de Bonnet-Myers, temos que $(M, \mathrm{~g})$ é compacta, e portanto Iso $(M, \mathrm{~g})$ é compacto. Assim, dado que $\Gamma$ é discreto e, pela Lema 1.2.2, é fechado em $I s o(M, \mathrm{~g})$, temos que $\Gamma$ é compacto, e portanto é finito.

Agora vamos supor que $k_{\bar{x}}>0$, para cada $\bar{x} \in X$. Logo temos que $k_{x}>0$ para cada $x \in M$. Agora, neste caso, temos que $I s o(M, \mathrm{~g})$ é compacto embora $M$ não seja compacta (vide [GM69], Teorema 3). Assim, dado que $\Gamma$ é discreto, temos que $\Gamma \subset I s o(M, \mathrm{~g})$ é finito.

2. Se $X$ é de curvatura negativa temos, pela Proposição 2.4.2, que $X$ é um good orbifold Riemanniano. Assim $X=M / \Gamma$, onde $(M, \mathrm{~g})$ é uma variedade simplesmente conexa completa e $\Gamma$ é um subgrupo discreto de Iso $(M, \mathrm{~g})$ que age propriamente sobre $M$. Agora, dado que $X$ é de curvatura negativa, temos, pelo Teorema de Hadamard, que $M$ é difeomorfa a $\mathbb{R}^{n}$, onde $n=\operatorname{dim}(M)$. Em especial temos que $M$ não pode ser compacta, e portanto, dado que $X$ é compacto, temos que $\Gamma$ é infinito. Então $\Gamma$ tem uma estrutura autónoma (vide $\left[\mathrm{ECH}^{+} 92\right]$, Teorema 3.4.1). Agora, todos os grupos infinitos com estrutura autónoma não podem ser de torsão (vide [ECH ${ }^{+}$92], Exemplo 2.5.12). Portanto $\Gamma$ é infinito e não é de torsão .

Assim temos a prova da proposição.

Observação 2.4.1 Se $(M, g)$ é uma variedade Riemanniana simplesmente conexa não compacta de curvatura positiva e $\Gamma$ é um subgrupo discreto de $\operatorname{Iso}(M, g)$ que age propriamente sobre $M$, então $\Gamma$ não age livremente. Para provar isto, primeiro observemos que $M / \Gamma$ é um good orbifold Riemanniano não compacto. Com efeito, pois se $M / \Gamma$ é um orbifold compacto e dado que Iso $(M, g)$ é compacto, temos que $M$ será compacta, que é um absurdo por hipótese. Agora, se $\Gamma$ age livremente sobre $M$, temos que $M / \Gamma$ é uma variedade Riemanniana de curvatura positiva, então $M / \Gamma$ é uma variedade simplesmente conexa (vide [GM69], Teorema 2), mas $\pi_{1}(M / \Gamma)=\Gamma$, e portanto temos um absurdo.

Corolário 2.4.1 Se $X=M / \Gamma$ é um orbifold Riemannino compacto com curvatura seccional positiva ou negativa, então $X$ admite uma geodésica fechada não trivial. 


\section{Demonstração.}

1. Vamos supor que $X$ tem curvatura positiva. Neste caso $X$ pode ser um good orbifold ou não. Se $X$ não é um good orbifold, então, por [GH06] (Teorema 5.1.1), temos que $X$ admite uma geodésica fechada não trivial. Agora, se $X$ é um good orbifold, então, pelo item número 1 da Proposição 2.4.2, temos que $\Gamma$ é finito. Logo $M$ é uma variedade Riemanniana compacta, pois $X$ é compacto. Logo $X$ admite uma geodésica fechada não trivial.

2. Agora supomos que $X$ tem curvatura seccional negativa. Assim, pela Proposição 2.4.1, temos que $X$ é um good orbifold Riemanniano. Logo, pelo item número 2 da Proposição 2.4.2, temos que existe $\omega$ em $\Gamma$ que é livre de torsão. Logo, dado que $X$ é compacto, temos que $\omega$ é uma isometria de tipo hiperbólico. Portanto, pelo Teorema 2.4.1, temos que $X$ admite uma geodésica fechada não trivial.

Antes da seguinte proposição vamos lembrar uma definição.

Definição 2.4.2 Sejam $M$ uma variedade Riemanniana e $\gamma: \mathbb{R} \rightarrow M$ uma geodésica. Dizemos que

$\gamma$ é uma linha em $M$ se e somente se $\left|\gamma^{\prime}(t)\right|=1$ e $d\left(\gamma\left(t_{1}\right), \gamma\left(t_{2}\right)\right)=\left|t_{1}-t_{2}\right|$, para todo par $t_{1}$ e $t_{2}$ em $\mathbb{R}$.

Proposição 2.4.3 Seja X um orbifold Riemanniano compacto.

1. Se X tem curvatura secional não-positiva, então X é um good orbifold Riemanniano e $\pi_{1}^{\text {orb }}(X)$ é infinito e não é de torsão.

2. Se X é um good orbifold e tem curvatura secional não negativa, então $\pi_{1}^{\text {orb }}(X)$ não pode ser infinito de torsão.

\section{Demonstração.}

1. Pela Proposição 2.4.1 temos que $X$ é um good orbifold Riemanniano. Logo $X=M / \Gamma$, onde $M$ é uma variedade completa e simplesmente conexa de curvatura seccional não-positiva e $\Gamma$ é um grupo de isometrias que age propriamente sobre $M$. Então temos que $M$ é um espaço de Hadamard, i.e., um espaço métrico simplesmente conexo, completo, e CAT (0). Então, por [Swe99] (Teorema 11), temos que existe $\omega$ em $\Gamma$ de ordem infinita. Logo $\Gamma$ é infinito e não é de torsão. 
2. Supomos que $X$ tem curvatura não-negativa. Dado que, por hipótese, $X$ é um good orbifold Riemanniano compacto, temos que $X=M / \Gamma$, onde $M$ é uma variedade Riemanniana completa e de curvatura seccional não-negativa. Se $M$ é compacta, então $\Gamma$ é finito, e portanto temos o resultado.

Agora vamos supor que $M$ não é compacta. Dado que $M$ possui curvatura não-negativa e é completa, temos, pelo teorema de descomposição de Toponogov [Top68], que $M$ pode se decompor de maneira única como $N \times \mathbb{R}^{k}$, onde $N$ é uma variedade Riemanniana completa de curvatura não-negativa que não possui linhas e $\mathbb{R}^{k}$ tem curvatura seccional zero. Agora, Cheeger e Gromoll [CG72] provam que se o grupo de isometrias de uma variedade completa $\bar{M}$ de curvatura não negativa é não compacto, então $\bar{M}$ possui uma linha. Assim temos que a descomposição isométrica única de $M$ como $N \times \mathbb{R}^{k}$, tem a propriedade que Iso $(M, \mathrm{~g})=\operatorname{Iso}\left(N, \mathrm{~g}_{1}\right) \times \operatorname{Iso}\left(\mathbb{R}^{k}, \mathrm{~g}_{2}\right)$, onde $N$ é de curvatura não-negativa, a curvatura de $\mathbb{R}^{k}$ é zero e Iso $\left(N, \mathrm{~g}_{1}\right)$ é compacto. Então podemos supor que $\Gamma=\Gamma_{N} \times \Gamma_{\mathbb{R}^{k}}$, onde $\Gamma_{N} \subset \operatorname{Iso}\left(N, \mathrm{~g}_{1}\right)$ e $\Gamma_{\mathbb{R}^{k}} \subset \operatorname{Iso}\left(\mathbb{R}^{k}, \mathrm{~g}_{2}\right)$. Dado que, por hipótese, $\Gamma$ é discreto, então $\Gamma_{N}$ e $\Gamma_{\mathbb{R}^{k}}$ são subgrupos discretos de Iso $\left(N, \mathrm{~g}_{1}\right)$ e Iso $\left(\mathbb{R}^{k}, \mathrm{~g}_{0}\right)$, respectivamente. Por outra parte, dado que $X$ é um good orbifold compacto, $N$ é uma variedade Riemanniana compacta, pois $\Gamma_{N}$ é finito, dado que Iso $\left(N, \mathrm{~g}_{1}\right)$ é compacto. Também, dado que $X$ é um good orbifold compacto, temos que $\mathbb{R}^{k} / \Gamma_{\mathbb{R}^{k}}$ é um good orbifold compacto. Portanto, como provamos antes, $\Gamma_{\mathbb{R}^{k}}$ possui um elemento livre de ordem infinita. Assim $\Gamma$ é infinito e não é de torsão.

Corolário 2.4.2 Seja X um orbifold Riemaniano compacto de curvatura não-positiva ou nãonegativa. Então $X$ possui uma geodésica fechada não trivial.

\section{Demonstração.}

1. Primeiro vamos supor que $X$ é um orbifold compacto e de curvatura não-positiva. Então, pela Proposição 2.4.1, temos que $X$ é um good orbifold Riemanniano, i.e., $X=M / \Gamma$, onde $M$ é uma variedade Riemanniana completa de curvatura não-positiva. Como provamos, temos que existe $\omega$ em $\Gamma$ de ordem infinita. Então, do Teorema 2.4.1, obtemos que existe uma geodésica não trivial em $X$. Assim temos a prova no primeiro caso.

2. Supomos que $X$ é de curvatura não-negativa. Se $X$ não é um good orbifiold, então, por [GH06] (Teorema 5.11), temos que $X$ admite uma geodésica fechada não trivial. Agora supomos que $X$ 
é um good orbifold Riemannino. Neste caso, pela Proposição 2.4.3, $\Gamma$ é finito ou é infinito sem torsão. Se $\Gamma$ é finito, o resultado segue-se do Teorema 2.4.1. Se $\Gamma$ é infinito sem torsão, então existe $\omega$ em $\Gamma$ que tem ordem infinita, e assim temos o resultado novamente pelo Teorema 2.4.1. 


\section{CAPÍTULO 3}

\section{RESULTADOS}

«Quando os escritores morrem, eles se transformam nos seus livros. O que, pensando bem, não deixa de ser uma forma interessante de reencarnação.»

-Jorge Luis Borges

Na seção 3.1 iremos apresentar os principais teoremas e resultados, vide Teorema 1, Teorema 2, Teorema 3, Corolário 1 e Observação 3.1.4.

Na seção 3.2 damos as ideias das demonstrações dos Teoremas 1, 2 e 3 e Corolário 1. Na seção 3.3 discutimos com mais detalhes os resultados sobre métrica da palavra e função deslocamento i.e, Observação 3.1.4.

Nas seções que se seguem, i.e, 3.4, 3.5, 3.6, 3.7 apresentamos as demonstrações detalhadas de tais resultados.

Por fim na seção 3.8, daremos uma prova alternativa dos resultados principais do capítulo 3, para o caso particular onde $M$ é o espaço Euclidiano $\mathbb{R}^{2}$ (com uma métrica não necessariamente 
euclidiana) e $\Gamma$ é um grupo de Coxeter Euclidiano. Como veremos na Proposição 3.8.1, neste caso particular poderemos inferir novas propriedades da família das infinitas geodésicas.

Neste capítulo assumiremos as seguintes convenções: para um good orbifold Riemanniano $M / \Gamma$, $(M, \mathrm{~g})$ sempre será uma variedade Riemanniana simplesmente conexa e completa (o que segue naturalmente se o quociente $M / \Gamma$ for compacto (vide [Bor92], Proposição 42). Observemos que neste caso $\Gamma$ age propriamente sobre $M$ e é um subgrupo discreto em Iso $(M, \mathrm{~g})$ (vide Proposição $1.2 \cdot 1)$

\subsection{Enunciados dos teoremas}

TEOREMA 1 (Teorema 3.4.1) Seja $X=M / \Gamma$ um good orbifold Riemanniano. Vamos supor que existe um subgrupo $\Gamma_{0}$ de $\Gamma$ com indice finito. Se $M / \Gamma_{0}$ admite infinitas geodésicas geometricamente distintas (vide definição 3.4.5) então temos que em X existem infinitas geodésicas fechadas geometricamente distintas.

Exemplo 3.1.1 (Cilindro) Sejam $(M, g)=\left(\mathbb{R}^{2}, g_{\text {can }}\right)$ e $X=\left\{(x, y, z) \in \mathbb{R}^{2}: x^{2}+y^{2}=\right.$ 1 e $z \geq 0\}$. Então $X$ pode ser visto como um good orbifold Riemanniano da forma $\mathbb{R}^{2} / \Gamma$, onde $\Gamma=\mathbb{Z} \rtimes \mathbb{Z}_{2}$, onde $\mathbb{Z}=\left\{\omega^{n}: n \in \mathbb{Z}\right\}$ e $\mathbb{Z}_{2}=\{r, e\}$, com $\omega$ e $r$ definidos da seguinte maneira

$$
\begin{array}{rllc}
\omega: & \mathbb{R}^{2} & \longrightarrow & \mathbb{R}^{2} \\
(x, y) & \longmapsto & (x+1, y) \\
r: \quad \mathbb{R}^{2} & \longrightarrow \mathbb{R}^{2} \\
(x, y) & \longmapsto(x,-y) .
\end{array}
$$

Observemos que a ação de $\Gamma$ sobre $\mathbb{R}^{2}$ pode ser vista como uma ação de $\mathbb{Z}_{2}$ sobre $\mathbb{R}^{2} / \mathbb{Z}$, i.e.,

$$
\begin{aligned}
\mathbb{Z}_{2} \times \mathbb{R}^{2} / \mathbb{Z} & \longrightarrow \mathbb{R}^{2} / \mathbb{Z} \\
(r,[(x, y)]) & \longmapsto
\end{aligned}
$$

Vejamos que esta ação está bem definida. Seja $\left(x_{1}, y_{1}\right) \in[(x, y)]$, então existe $n \in \mathbb{Z}$ tal que $x_{1}=x+n$ e $y_{1}=y$. Agora

$$
r\left(x_{1}, y_{1}\right)=\left(x_{1},-y_{1}\right)=(x+n,-y) \in[r(x, y)] .
$$


Portanto a ação está bem definida.

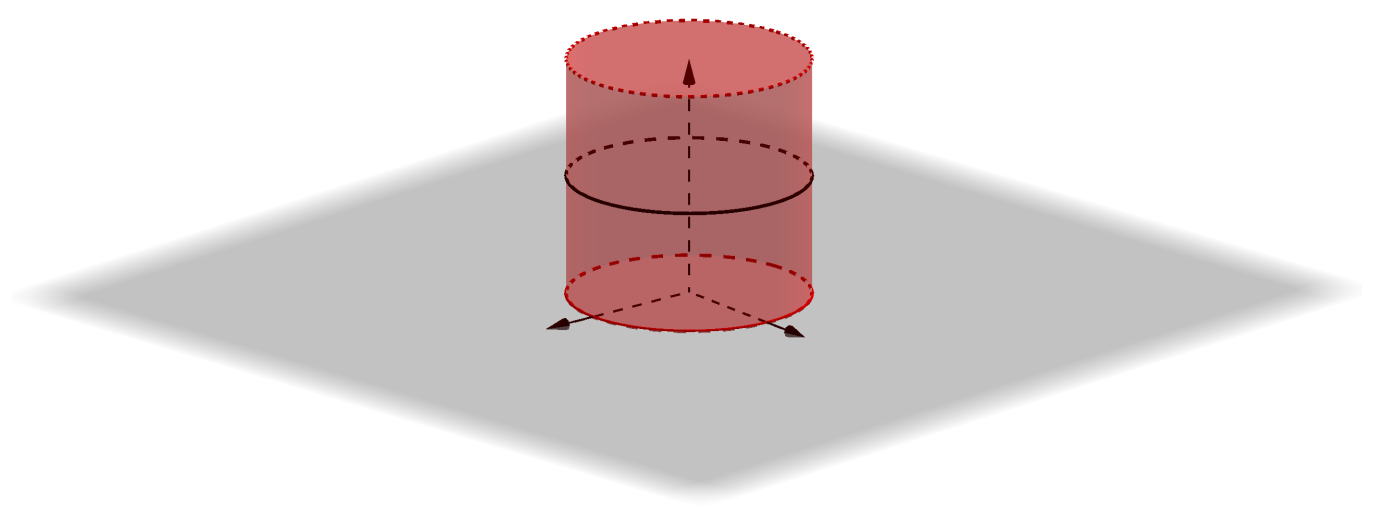

Figura 3.1: $O$ orbifold $X=\left\{(x, y, z) \in \mathbb{R}^{2}: x^{2}+y^{2}=1\right.$ e $\left.z \geq 0\right\}$.

Neste caso temos que $\mathbb{Z}$ é de índice finito em $\Gamma$. Além disso, o good orbifold $\mathbb{R}^{2} / \mathbb{Z}$ possui infinitas geodésicas fechadas geometricamente distintas. Portanto $X$ possui infinitas geodésicas fechadas geometricamente distintas.

TEOREMA 2 (Teorema 3.5.1) Seja $X=M / \Gamma$ um good orbifold Riemanniano compacto, onde $M$ é uma variedade Riemanniana com $\operatorname{dim}(M)>1$ e $\Gamma$ é um grupo infinito. Suponhamos que existe um subgrupo $\Gamma_{0}$ abeliano e de indice finito em $\Gamma$, então $X$ admite uma família

$$
\left\{\left(\omega_{i},\left.\alpha_{i}\right|_{[0,1]}\right)\right\}_{i \in \mathbb{N}}
$$

de geodésicas fechadas geometricamente distintas dois a dois.

Observação 3.1.1 Seja $\Gamma$ um grupo grupo abeliano finitamente gerado, então temos que

$$
\Gamma_{0} \approx T\left(\Gamma_{0}\right) \oplus \mathbb{Z}^{k}
$$

Onde $T\left(\Gamma_{0}\right)$ é o subgrupo finito dos elementos de torção de $\Gamma$. Neste caso $k$ é chamado o posto de $\Gamma_{0}$ ou também é conhecido como o número de Betti de $\Gamma_{0}$.

Observação 3.1.2 Em [GH06] (Teorema 5.2.4), Guruprasad e Haefliger provam que todo good orbifold compacto $X=M / \Gamma$, onde $\Gamma$ é um grupo infinito abeliano, possui infinitas geodésicas fechadas geometricamente distintas. Assim, misturando o resultado de Guruprasad e Haefliger e o Teorema 1, temos o resultado do Teorema 2. Agora, é bom dizer que nossa prova (que pode ser vista como alternativa) será útil na hora da demostração do Teorema 3 e da Proposição 2. 
Exemplo 3.1.2 (Pillow) Considere $(M, \mathrm{~g})=\left(\mathbb{R}^{2}, \mathrm{~g}_{\text {can }}\right)$ e $\Gamma=\mathbb{Z}^{2} \rtimes \mathbb{Z}_{2}$, onde $\mathbb{Z}^{2}$ é gerado pelas seguintes isometrias

$$
\begin{aligned}
\omega_{1}: \mathbb{R}^{2} & \longrightarrow \mathbb{R}^{2} \\
(x, y) & \longmapsto(x+1, y) \\
\omega_{2}: \quad \mathbb{R}^{2} & \longrightarrow \mathbb{R}^{2} \\
(x, y) & \longmapsto(x, y+1) .
\end{aligned}
$$

$E \mathbb{Z}_{2}$ é gerado pela seguinte isometria

$$
\begin{aligned}
r: \mathbb{R}^{2} & \longrightarrow \mathbb{R}^{2} \\
(x, y) & \longmapsto(-x,-y) .
\end{aligned}
$$

Observemos que a ação de $\Gamma$ sobre $\mathbb{R}^{2}$ pode ser vista como a ação de $\mathbb{Z}_{2}$ sobre $\mathbb{R}^{2} / \mathbb{Z}^{2}$, i.e.,

$$
\begin{aligned}
\mathbb{Z}_{2} \times \mathbb{R}^{2} / \mathbb{Z}^{2} & \longrightarrow \mathbb{R}^{2} / \mathbb{Z}^{2} \\
(r,[(x, y)]) & \longmapsto[r(x, y)]
\end{aligned}
$$

Vejamos que esta ação está bem definida. Com efeito, seja $\left(x_{1}, y_{1}\right) \in[(x, y)]$, então existe um par $l, k$ em $\mathbb{Z}$ tal que $x_{1}=x+l$ e $y_{1}=y+k$ e portanto,

$$
r\left(x_{1}, y_{1}\right)=\left(-x_{1},-y_{1}\right)=(-(x+l),-(y+k)) \in[(-x,-y)]=[r(x, y)] .
$$

Observemos que o toro tem infinitas geodésicas fechadas geometricamente distintas e portanto, dado que $\mathbb{Z}^{2}$ é de indice finito em $\Gamma$, temos que $X=\mathbb{R}^{2} / \Gamma$ admite infinitas geodésicas geometricamente distintas. Alé, observemos que em $X=\mathbb{R}^{2} / \Gamma$ temos quatros pontos singulares.
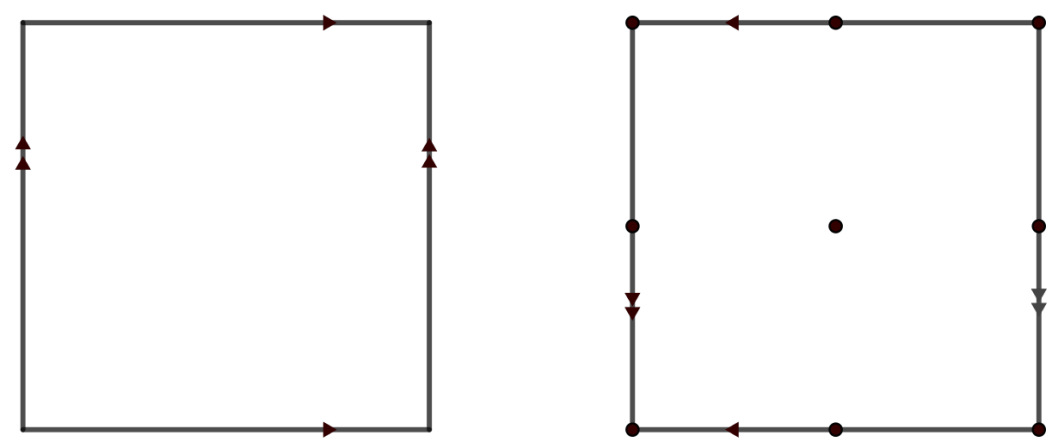

Figura 3.2: $\mathbb{R}^{2} / \mathbb{Z}^{2}$ e $\mathbb{R}^{2} / \Gamma$. 
Observação 3.1.3 Observe que a hipótese do Teorema 2 é bem mais geral do que os exemplos listados acima. Mais precisamente, somente exigimos que o grupo $\Gamma$ seja virtualmente abeliano. $O$ grupo $\Gamma$, em particular, não precisa ser um produto semi-direto $\tilde{\Gamma} \times \mathbb{Z}^{k}$ (onde $\tilde{\Gamma}$ é finito). Isto implica que técnicas utilizadas na demonstração do Corolário 1 (e.g, considerar ação de $\tilde{\Gamma}$ em $M / \mathbb{Z}^{k}$ ) não podem ser utilizadas.

TEOREMA 3 (Teorema 3.6.1) Seja $X=M / \Gamma$ um good orbifold Riemanniano compacto, onde $M$ é uma variedade Riemanniana tal que $\operatorname{dim}(M)>1$ e $\Gamma$ é um grupo infinito. Vamos supor que existe um subgrupo $\Gamma_{0}$ de $\Gamma$ que é abeliano e de índice finito em $\Gamma$ e tal que o posto de $\Gamma_{0}$ é maior ou igual que 2. Então X admite uma família $\left\{\left(\omega_{i},\left.\alpha_{i}\right|_{[0,1]}\right)\right\}_{i \in \mathbb{N}}$ de geodésicas fechadas geometricamente distintas dois a dois. E se definimos $X_{0}=M / \Gamma_{0}$, então $\left\{\mathcal{L}_{X_{0}}\left(\left.\alpha_{i}\right|_{[0,1]}\right)\right\}_{i \in \mathbb{N}}$ é uma sequência real não limitada. Em particular, a sequência $\left\{\left|\omega_{i}\right|\right\}_{i \in \mathbb{N}}$ é não limitada.

Exemplo 3.1.3 (Pillow) Observemos que o exemplo anterior (vide Exemplo 3.1.2) satisfaz as hipôteses do teorema.

Corolário 1 Seja $X=M / \Gamma$ um good orbifold Riemanniano compacto, onde $M$ é uma variedade Riemanniana tal que $\operatorname{dim}(M)>1$ e $\Gamma=\tilde{\Gamma} \ltimes \mathbb{Z}^{k}$, com $k \geq 2$ e $\tilde{\Gamma}$ é um grupo finito. Então temos que o orbifold Riemanniano $X=M / \Gamma$ admite uma famılia $\left\{\left(\omega_{i},\left.\alpha_{i}\right|_{[0,1]}\right)\right\}_{i \in \mathbb{N}}$ de geodésicas fechadas geometricamente distintas e $\left\{\mathcal{L}_{X}\left(\left.\alpha_{i}\right|_{[0,1]}\right)\right\}_{i \in \mathbb{N}}$ é uma sequência real não limitada.

Observação 3.1.4 (Métrica da Palavra e Função Deslocamento) Na seção 3.3 (vide também seção 3.7) iremos discutir (no caso em que o grupo é abeliano) uma fórmula que relaciona a métrica da palavra com a função deslocamento, vide Proposição 2 (Proposição 3.7.1).

Tal fórmula permitirá a demonstração da Proposição 3 (vide também a Proposição 3.7.2) a qual deve ser vista como uma melhoria do Colorário 1. Em particular, na Proposição 3 descrevemos de forma mais precisa o crescimento das geodésicas, e.g., relacionando seus comprimentos com o a métrica da palavra.

\subsection{Ideias das Provas dos Teoremas e das Proposições}

\subsubsection{Ideia da Prova do Teorema 1 (3.4.1).}

Por hipótese existe uma sequência infinita $\left\{\left(\omega_{i},\left.\alpha_{i}\right|_{[0,1]}\right)\right\}_{i \in \mathbb{N}}$ de geodésicas fechadas geometricamente distintas dois a dois em $M / \Gamma_{0}$ (vide definição 3.4.5). Afirmamos existem infinitas classes em $M / \Gamma$ tal que cada classe contém no máximo um número finito de elementos de $\left\{\left(\omega_{i},\left.\alpha_{i}\right|_{[0,1]}\right)\right\}_{i \in \mathbb{N}}$. 
E vamos provar a veracidade da afirmação pelo absurdo, i.e., vamos supor que existe apenas um número finito de classes em $M / \Gamma$ ou existe pelo menos uma classe em $M / \Gamma$ que contém infinitos elementos de $\left\{\left(\omega_{i},\left.\alpha_{i}\right|_{[0,1]}\right)\right\}_{i \in \mathbb{N}}$. Assim, temos que existe uma subsequência de $\left\{\left(\omega_{i},\left.\alpha_{i}\right|_{[0,1]}\right)\right\}_{i \in \mathbb{N}}$ onde todos seus termos são geodésicas fechadas geometricamente iguais em $X$. Logo, por simplicidade, podemos supor que tal subsequência é a mesma sequência $\left\{\left(\omega_{i},\left.\alpha_{i}\right|_{[0,1]}\right)\right\}_{i \in \mathbb{N}}$.

Fixemos $\left(\omega_{1},\left.\alpha_{1}\right|_{[0,1]}\right)$, e seja $j>1$, qualquer. Dado que $\left(\omega_{1},\left.\alpha_{1}\right|_{[0,1]}\right)$ e $\left(\omega_{j},\left.\alpha_{j}\right|_{[0,1]}\right)$ são geometricamente iguais em $X$, então (vide Proposição 3.4.7) existem $\omega_{j_{1}} \in \Gamma \backslash \Gamma_{0}$ e números reais $a_{j_{1}}$ e $b_{j_{1}}$ tais que

$$
\alpha_{1}(t)=\omega_{j_{1}} \alpha_{j}\left(a_{j_{1}} t+b_{j_{1}}\right) \text {, para todo } t \in \mathbb{R}
$$

Então, para $k>1$ e $j>1$, obtemos

$$
\omega_{j_{1}} \alpha_{j}\left(a_{j_{1}} t+b_{j_{1}}\right)=\alpha_{1}(t)=\omega_{k_{1}} \alpha_{k}\left(a_{k_{1}} t+b_{k_{1}}\right) \text {, para todo } t \in \mathbb{R} \text {. }
$$

Assim, afirmamos que se $j \neq k$ então $\omega_{j_{1}} \neq \omega_{k_{1}}$. Pois se acontece o contrário, vamos obter da equação (3.2), que $\left(\omega_{k},\left.\alpha_{k}\right|_{[0,1]}\right)$ e $\left(\omega_{j},\left.\alpha_{j}\right|_{[0,1]}\right)$ são geometricamente iguais em $M / \Gamma_{0}$, que é um absurdo, pois por hipótese elas são geometricamente distintas em $M / \Gamma_{0}$. Portanto, os elementos da família $\left\{\omega_{j_{1}}\right\}_{j>1}$ são todos distintos.

Agora, temos dois casos, e cada um deles depende somente da cardinalidade de $\Gamma_{0}$, pois $\Gamma_{0}$ é de índice finito em $\Gamma$ :

1. $\Gamma \backslash \Gamma_{0}$ é finito;

2. $\Gamma \backslash \Gamma_{0}$ é infinito.

No primeiro caso vamos obter um absurdo, pois $\left\{\omega_{j_{1}}\right\}_{j>1}$ é uma família infinita. Agora vamos para o segundo caso. Dado que $\Gamma_{0}$ é de índice finito em $\Gamma$, existe um conjunto $\left\{\bar{\omega}_{1}, \bar{\omega}_{2}, \ldots, \bar{\omega}_{l}\right\} \subset \Gamma \backslash \Gamma_{0}$ tal que

$$
\Gamma=\Gamma_{0} \dot{\sqcup} \bar{\omega}_{1} \Gamma_{0} \dot{ப} \ldots \dot{\dot{\omega}} \bar{\omega}_{l} \Gamma_{0}
$$

Portanto, temos que, para cada $\omega_{i} \in\left\{\omega_{j_{1}}\right\}_{j>1}, \omega_{i}=\bar{\omega}_{i} \tilde{\omega}_{i}$, onde $\bar{\omega}_{i}$ é um elemento de $\left\{\bar{\omega}_{1}, \bar{\omega}_{2}, \ldots, \bar{\omega}_{l}\right\}$ e $\tilde{\omega}_{i} \in \Gamma_{0}$. Assim, pela equação (3.2) e dado que a família $\left\{\left(\omega_{i},\left.\alpha_{i}\right|_{[0,1]}\right)\right\}_{i \in \mathbb{N}}$ é de geodésicas fechadas geometricamente distintas em $M / \Gamma_{0}$, obtemos que existe uma aplicação injetora de $\left\{\omega_{j_{1}}\right\}_{j>1}$ em $\left\{\bar{\omega}_{1}, \bar{\omega}_{2}, \ldots, \bar{\omega}_{l}\right\} \subset \Gamma \backslash \Gamma_{0}$, i.e., se $\omega_{i} \neq \omega_{j}$, então $\bar{\omega}_{i} \neq \bar{\omega}_{j}$. Mas isso é um absurdo por cardinalidade. Dado que em cada um dos casos obtivemos uma contradição, então existem infinitas classes em $M / \Gamma$ tal que cada classe contém no máximo um número finito de elementos de $\left\{\left(\omega_{i},\left.\alpha_{i}\right|_{[0,1]}\right)\right\}_{i \in \mathbb{N}}$. Logo 
existe uma subsequência $\left\{\left(\omega_{i_{k}},\left.\alpha_{i_{k}}\right|_{[0,1]}\right)\right\}_{k \in \mathbb{N}}$ de $\left\{\left(\omega_{i},\left.\alpha_{i}\right|_{[0,1]}\right)\right\}_{i \in \mathbb{N}}$ tal que todos os elementos de $\left\{\left(\omega_{i_{k}},\left.\alpha_{i_{k}}\right|_{[0,1]}\right)\right\}_{k \in \mathbb{N}}$ são geodésicas fechadas geometricamente distintas dois a dois em $X$.

temos a veracidade do teorema.

\subsubsection{Ideia da Prova do Teorema 2 (3.5.1).}

Dado que $\Gamma_{0}$ é de índice finito em $\Gamma$ e $X$ é compacto então temos que $M / \Gamma_{0}$ também é compacto. Assim, vamos ter que $\Gamma_{0}$ é finitamente gerado. E portanto,

$$
\Gamma_{0} \simeq T\left(\Gamma_{0}\right) \oplus \mathbb{Z}^{k}
$$

Dado que $\Gamma$ é infinito e $\Gamma_{0} \subset \Gamma$ é de índice finito, temos que $k \geq 1$, e também vamos obter que $M / \mathbb{Z}^{k}$ é uma variedade Riemanniana compacta. Agora, a prova do teorema será obtida a partir do valor de $k$. No caso de $k=1$ temos por [BH84] que a variedade $M / \mathbb{Z}$ admite infinitas geodésicas geometricamente distintas. Assim, dado que $\mathbb{Z}$ é de índice finito em $\Gamma$, podemos aplicar o Teorema 1 para obter que $M / \Gamma$ admite uma família infinita de geodésicas fechadas geometricamente distintas. No caso de $k \geq 2$ e sendo $\mathcal{A}=\left\{v_{1}, \ldots, v_{k}\right\}$ uma base para $\mathbb{Z}^{k}$, para cada $i \geq 1$ definimos

$$
\omega_{i}:=v_{1}+v_{2}+\cdots+v_{k-1}+i v_{k} .
$$

Logo cada $\omega_{i}$ é um elemento hiperbólico (vide definição 2.2.3). Assim, pelo item número 2 do Teorema 2.4.1, para cada $i \in \mathbb{N}$, vamos obter uma geodésica fechada

$$
\left(\omega_{i},\left.\alpha_{i}\right|_{[0,1]}\right) \text {. }
$$

Onde, em particular, para cada $i \in \mathbb{N}$, temos que $\mathcal{L}\left(\left.\alpha_{i}\right|_{[0,1]}\right)=\left|\omega_{i}\right|$ (vide definição 2.2.2). Logo temos uma família infinita de geodésicas fechadas em $M / \mathbb{Z}^{k}$

$$
\left\{\left(\omega_{i},\left.\alpha_{i}\right|_{[0,1]}\right)\right\}_{i \in \mathbb{N}}
$$

Agora vamos provar que todos os elementos da família $\left\{\left(\omega_{i},\left.\alpha_{i}\right|_{[0,1]}\right)\right\}_{i \in \mathbb{N}}$ são geometricamente distintos dois a dois em $M / \mathbb{Z}^{k}$. Primeiramente, podemos observar que (vide Lema 3.5.2):

1. se $i \neq j$, então $\omega_{i}^{n} \neq \omega_{j}^{m}$, para todo par $n, m$ em $\mathbb{Z}^{*}$;

2. para cada $i \in \mathbb{N}$, a geodésica fechada $\left(\omega_{i},\left.\alpha_{i}\right|_{[0,1]}\right)$ não possui geodésica primitiva em $M / \mathbb{Z}^{k}$ distinta dela mesma. 
Supomos que para $i \neq j$ temos que que $\left(\omega_{i},\left.\alpha_{i}\right|_{[0,1]}\right)$ e $\left(\omega_{j},\left.\alpha_{j}\right|_{[0,1]}\right)$ são geometricamente iguais em $M / \mathbb{Z}^{k}$. Então, dado que $\left(\omega_{i},\left.\alpha_{i}\right|_{[0,1]}\right)$ e $\left(\omega_{j},\left.\alpha_{j}\right|_{[0,1]}\right)$ não possuem geodésicas primitivas (vide a definição 3.7.1), existem $\omega \in \mathbb{Z}^{k}$ e $b \in \mathbb{R}$ tais que

$$
\omega \alpha_{i}( \pm t+b)=\alpha_{j}(t) \text { para todo } t \in \mathbb{R}
$$

Assim, precisamos trabalhar em dois casos:

1. Supomos que $\omega \alpha_{i}(t+b)=\alpha_{j}(t)$, para todo $t \in \mathbb{R}$. Neste caso vamos obter que $\left(\omega_{j},\left.\omega \alpha_{i}\right|_{[0,1]}\right)$ é uma geodésica fechada. Assim, dado que $\left(\omega_{i},\left.\alpha_{i}\right|_{[0,1]}\right)$ também é uma geodésica fechada, temos que

$$
\omega_{j} \omega \alpha_{i}(t)=\omega \alpha_{i}(t+1)=\omega \omega_{i} \alpha_{i}(t), \text { para todo } t \in \mathbb{R} .
$$

Assim, dado que $\mathbb{Z}^{k}$ é abeliano e que age livremente sobre $M$, temos que $\omega_{i}=\omega_{j}$, que é um absurdo, pois $i \neq j$.

2. Supomos que $\omega \alpha_{1}(-t+b)=\alpha_{2}(t)$, para todo $t \in \mathbb{R}$. Neste caso obtemos que $\left(\omega_{j}^{-1},\left.\omega \alpha_{i}\right|_{[0,1]}\right)$ é uma geodésica fechada. Agora, dado que $\left(\omega_{i},\left.\alpha_{i}\right|_{[0,1]}\right)$ também é uma geodésica fechada, temos que

$$
\omega_{j}^{-1} \omega \alpha_{i}(t)=\omega \alpha_{i}(t+1)=\omega \omega_{i} \alpha_{i}(t), \text { para todo } t \in \mathbb{R}
$$

Logo, dado que $\mathbb{Z}^{k}$ é abeliano e que age livremente sobre $M$, temos que $\omega_{i}=\omega_{j}^{-1}$, que é um absurdo, pois $\omega_{i}^{n} \neq \omega_{j}^{m}$, para todo par $n, m$ em $\mathbb{Z}^{*}$.

Assim, todas as geodésicas fechadas de $\left\{\left(\omega_{i},\left.\alpha_{i}\right|_{[0,1]}\right)\right\}_{i \in \mathbb{N}}$ são geometricamente distintas em $M / \mathbb{Z}^{k}$. Logo, dado que $\mathbb{Z}^{k}$ é de índice finito em $\Gamma$ e aplicando o Teorema 1 , obtemos que $\left\{\left(\omega_{i},\left.\alpha_{i}\right|_{[0,1]}\right)\right\}_{i \in \mathbb{N}}$ admite uma subsequência

$$
\left\{\left(\omega_{i_{k}},\left.\alpha_{i_{k}}\right|_{[0,1]}\right)\right\}_{k \in \mathbb{N}}
$$

de geodésicas fechadas geometricamente distintas em $M / \Gamma$. E assim temos o resultado do teorema.

\subsubsection{Ideia da Prova do Teorema 3 (3.6.1).}

Antes de dar uma ideia do Teorema 3, vamos dar a seguinte proposição que será útil na prova.

Proposição 1 (Proposição 3.6.1) Seja $X=M / \Gamma$ um good orbifold compacto onde $\Gamma$ é um grupo abeliano. Supomos que existe uma família $\left\{\left(\omega_{i},\left.\alpha_{i}\right|_{[0,1]}\right)\right\}_{i \in \mathbb{N}}$ de geodésicas primitivas geometrica- 
mente distintas em $X$ tal que $\omega_{i} \neq \omega_{j}$, para todo par $i, j$ com $i \neq j$. Então

$$
\left\{\mathcal{L}\left(\left.\alpha_{i}\right|_{[0,1]}\right)\right\}_{i \in \mathbb{N}}
$$

é uma sequência real não limitada.

Agora vamos dar uma ideia da prova do Teorema 3.

Seja $\mathcal{A}=\left\{v_{1}, \ldots, v_{k}\right\}$ uma base para $\mathbb{Z}^{k}$. E para cada $i \geq 1$ definimos

$$
\omega_{i}:=v_{1}+v_{2}+\cdots+v_{k-1}+i v_{k} .
$$

Cada $\omega_{i}$ gera uma geodésica fechada $\left(\omega_{i},\left.\alpha_{i}\right|_{[0,1]}\right)$, e, pelo Teorema 2 , temos que a família $\left(\omega_{i},\left.\alpha_{i}\right|_{[0,1]}\right)$ admite uma subsequência $\left\{\left(\omega_{i_{k}},\left.\alpha_{i_{k}}\right|_{[0,1]}\right)\right\}_{k \in \mathbb{N}}$ de geodésicas fechadas geometricamente distintas em $M / \Gamma$. Assim temos a prova da primeira parte do teorema.

Para provar a segunda parte do teorema basta mostrar que se $\left\{\left(\bar{\omega}_{i},\left.\alpha_{i}\right|_{\left[0, t_{i}\right]}\right)\right\}_{i \in \mathbb{N}}$ é uma família de geodésicas primitivas associadas à família $\left\{\left(\omega_{i},\left.\alpha_{i}\right|_{[0,1]}\right)\right\}_{i \in \mathbb{N}}$ em $X_{0}$, então

$$
\lim _{i \rightarrow \infty} \mathcal{L}_{X_{0}}\left(\left.\alpha_{i}\right|_{[0,1]}\right)=\lim _{i \rightarrow \infty} \mathcal{L}\left(\left.\alpha_{i}\right|_{\left[0, t_{i}\right]}\right) \rightarrow \infty
$$

Afirmamos que para cada par de índices $j, l$ em $\mathbb{N}$, com $j \neq l$, temos que $\bar{\omega}_{j} \neq \bar{\omega}_{l}$. Vamos provar esta afirmação pelo absurdo, i.e., vamos supor que $\bar{\omega}_{j}=\bar{\omega}_{l}$, para alguns $j \neq l$. Dado que $\left(\bar{\omega}_{j},\left.\alpha_{j}\right|_{\left[0, t_{j}\right]}\right)$ e $\left(\bar{\omega}_{l},\left.\alpha_{l}\right|_{\left[0, t_{l}\right]}\right)$ são geodésicas fechadas primitivas associadas às geodésicas fechadas $\left(\omega_{j},\left.\alpha_{j}\right|_{[0,1]}\right)$ e $\left(\omega_{k},\left.\alpha_{l}\right|_{[0,1]}\right)$, respectivamente, existem $m_{j}, k_{j}, m_{l}, k_{l}$ em $\mathbb{N}$, tais que (vide Proposição 3.4.5)

$$
\bar{\omega}_{j}^{m_{j}}=\omega_{j}^{k_{j}} \text { e } \bar{\omega}_{l}^{m_{l}}=\omega_{l}^{k_{l}} .
$$

Assim, dado que estamos supondo que $\bar{\omega}_{j}=\bar{\omega}_{l}$, temos que

$$
\omega_{j}^{m_{l} k_{l} k_{j} k_{j}}=\omega_{l}^{m_{j} k_{j} k_{l} k_{l}} .
$$

Mas isso é um absurdo (vide Lema 3.5.2). Portanto $\bar{\omega}_{j} \neq \bar{\omega}_{j}$, para $j \neq l$, i.e., todos os elementos da família $\left\{\bar{\omega}_{i}\right\}_{i \in \mathbb{N}}$ são dois a dois distintos.

Assim, a família $\left\{\left(\bar{\omega}_{i},\left.\alpha_{i}\right|_{\left[0, t_{i}\right]}\right)\right\}_{i \in \mathbb{N}}$ satisfaz as hipóteses da Proposição 1 no orbifold Riemanniano compacto $M / \Gamma_{0}$. Então temos que $\left\{\mathcal{L}\left(\left.\alpha_{i}\right|_{\left[0, t_{i}\right]}\right)\right\}_{i \in \mathbb{N}}$ é uma sequência real não limitada, e portanto temos a segunda parte do teorema. Observe que também temos que a sequência $\left\{\left|\omega_{i}\right|\right\}_{i \in \mathbb{N}}$ é não 
limitada.

\subsubsection{Prova do Corolário 1}

Demonstração. Na ideia da prova da primeira parte do Teorema 3.2.3, construímos uma sequência

$$
\left\{\left(\omega_{i},\left.\alpha_{i}\right|_{[0,1]}\right)\right\}_{i \in \mathbb{N}}
$$

de geodésicas fechadas em $X$ a partir de uma base $\mathcal{A}=\left\{v_{1}, \ldots, v_{k}\right\}$ de $\mathbb{Z}^{k}$, onde $\omega_{i}:=v_{1}+$ $v_{2}+\cdots+v_{k-1}+i v_{k}$ e $\mathcal{L}\left(\left.\alpha_{i}\right|_{[0,1]}\right)=\left|\omega_{i}\right|$. Agora, dado que $\mathbb{Z}^{k}$ é de índice finito em $\Gamma$, temos, pelo Teorema 3.4.1, que existe uma subsequência $\left\{\left(\omega_{i_{k}},\left.\alpha_{i_{k}}\right|_{[0,1]}\right)\right\}_{k \in \mathbb{N}}$ de $\left\{\left(\omega_{i},\left.\alpha_{i}\right|_{[0,1]}\right)\right\}_{i \in \mathbb{N}}$, onde todas as geodésicas fechadas de $\left\{\left(\omega_{i_{k}},\left.\alpha_{i_{k}}\right|_{[0,1]}\right)\right\}_{k \in \mathbb{N}}$ são geometricamente distintas em $X$. Assim temos a primeira parte da corolário.

Seja $\left\{\left(\bar{\omega}_{i},\left.\alpha_{i}\right|_{\left[0, t_{i}\right]}\right)\right\}_{i \in \mathbb{N}}$ uma família de geodésicas fechadas primitivas de $\left\{\left(\omega_{i},\left.\alpha_{i}\right|_{[0,1]}\right)\right\}_{i \in \mathbb{N}}$. Neste caso vamos ter que cada geodésica fechada $\left(\omega_{i},\left.\alpha_{i}\right|_{[0,1]}\right)$ não possui geodésica primitiva em $M / \mathbb{Z}^{k}$ (vide Teorema 3.6.1). Portanto, cada $\bar{\omega}_{i}$ é um elemento de $\Gamma \backslash \mathbb{Z}^{k}$. Dado que $\Gamma=\tilde{\Gamma} \ltimes \mathbb{Z}^{k}$, existem $\tilde{\omega}_{i} \in \tilde{\Gamma}$ e $\hat{\omega}_{i} \in \mathbb{Z}^{k}$ tais $\bar{\omega}_{i}=\tilde{\omega}_{i} \hat{\omega}_{i}$. Também temos que não pode acontecer que $\bar{\omega}_{i} \in \tilde{\Gamma}$, pois dado que $\tilde{\Gamma}$ é finito, vamos ter que $\bar{\omega}_{i}$ é de ordem finita, e isso implica que $\omega_{i}$ é de ordem finita, que é um absurdo (vide item número dois da Observação 3.4.6).

Afirmamos que o espaço de órbitas $M / \Gamma$ é equivalente ao espaço de órbitas $\left[M / \mathbb{Z}^{k}\right] / \tilde{\Gamma}$, i.e., a ação

$$
\begin{aligned}
\mu: \tilde{\Gamma} \times M / \mathbb{Z}^{k} & \longrightarrow M / \mathbb{Z}^{k} \\
(\tilde{\omega},[x]) & \longrightarrow[\tilde{\omega}(x)]
\end{aligned}
$$

está bem definida. Observemos que tem sentido falar de uma ação de $\tilde{\Gamma}$ sobre $M / \mathbb{Z}^{k}$ pois $M / \mathbb{Z}^{k}$ é uma variedade Riemanniana.

Primeiro vamos provar que (3.4) está bem definida. Com efeito, sejam $\tilde{\omega} \in \tilde{\Gamma}, \omega \in \mathbb{Z}^{k}$ e $x \in M$, então temos que $\tilde{\omega} \omega(x)=\hat{\omega} \tilde{\omega}(x)$ para algum $\hat{\omega} \in \mathbb{Z}^{k}$, pois $\mathbb{Z}^{k}$ é um subgrupo normal de $\Gamma$. Agora vamos provar que (3.4) define uma ação. Sejam $\tilde{\omega}_{1}$ e $\tilde{\omega}_{2}$ em $\tilde{\Gamma}$ e $x \in M$, então

$$
\mu\left(\tilde{\omega}_{1}, \mu\left(\tilde{\omega}_{2},[x]\right)\right)=\mu\left(\tilde{\omega}_{1},\left[\tilde{\omega}_{2}(x)\right]\right)=\left[\tilde{\omega}_{1} \tilde{\omega}_{2}(x)\right]=\mu\left(\tilde{\omega}_{1} \tilde{\omega}_{2},[x]\right) .
$$

Sejam $e$ o elemento identidade em $\tilde{\Gamma}$ e $x \in M$, então

$$
\mu(e,[x])=[x] .
$$


E assim temos que (3.4) define uma ação de $\tilde{\Gamma}$ sobre a variedade $M / \mathbb{Z}^{k}$.

Definamos $\pi_{0}: M \rightarrow M / \mathbb{Z}^{k}$ como a aplicação quociente da ação de $\mathbb{Z}^{k}$ sobre $M$. Então temos que a família $\left\{\left(\omega_{i},\left.\alpha_{i}\right|_{[0,1]}\right)\right\}_{i \in \mathbb{N}}$ de geodésicas fechadas induz uma família $\left\{\pi_{0}\left(\alpha_{i}\right)\right\}_{i \in \mathbb{N}}$ de geodésicas periódicas em $M / \mathbb{Z}^{k}$. Seja $i$ qualquer elemento em $\mathbb{N}$, e dado que $\left(\bar{\omega}_{i},\left.\alpha_{i}\right|_{\left[0, t_{i}\right]}\right)$ é uma geodésica fechada primitiva de $\left(\omega_{i},\left.\alpha_{i}\right|_{\left[0, t_{i}\right]}\right)$, então, supondo que $\bar{\omega}_{i}=\tilde{\omega}_{i} \hat{\omega}_{i}$, onde $\tilde{\omega}_{i} \in \tilde{\Gamma}$ e $\hat{\omega}_{i} \in \mathbb{Z}^{k}$, temos

$$
\bar{\omega}_{i} \alpha_{i}(t)=\tilde{\omega}_{i} \hat{\omega}_{i} \alpha_{i}(t)=\alpha_{i}\left(t+t_{i}\right), \text { para todo } t \in \mathbb{R} \text { (vide Proposição 3.4.1). }
$$

Assim, pela ação (3.4), a família de geodésicas primitivas $\left\{\left(\bar{\omega}_{i},\left.\alpha_{i}\right|_{\left[0, t_{i}\right]}\right)\right\}_{i \in \mathbb{N}}$ torna-se na família $\left\{\left(\tilde{\omega}_{i},\left.\pi_{0}\left(\alpha_{i}\right)\right|_{\left[0, t_{i}\right]}\right)\right\}_{i \in \mathbb{N}}$, onde $\tilde{\omega}_{i} \in \tilde{\Gamma}$, pois estamos supondo que $\bar{\omega}_{i}=\tilde{\omega}_{i} \hat{\omega}_{i}, \operatorname{com} \hat{\omega}_{i} \in \mathbb{Z}^{k}$.

Dado que $\bar{\Gamma}$ é finito, temos que cada $\tilde{\omega} \in \tilde{\Gamma}$ é de ordem finita. Logo, usando isto e que $\tilde{\Gamma}$ é um grupo finito, temos que existe $l \in \mathbb{N}$ tal que $\tilde{\omega}^{l}=e$, para cada $\tilde{\omega} \in \tilde{\Gamma}$. Em especial temos que cada $\tilde{\omega}_{i}^{l}=e$. Seja $m_{i}$ a ordem de $\tilde{\omega}_{i}$, então

$$
\left.\left.\left.\pi_{0}\left(\alpha_{i}\right)(t)\right|_{\left[0, t_{i}\right]} * \tilde{\omega}_{i} \pi_{0}\left(\alpha_{i}\right)(t)\right|_{\left[0, t_{i}\right]} * \cdots * \tilde{\omega}_{i}^{m_{i}-1} \pi_{0}\left(\alpha_{i}\right)(t)\right|_{\left[0, t_{i}\right]}
$$

é uma geodésica periódica em $M / \mathbb{Z}^{k}$ (vide Proposição 3.4.6). Mais ainda, temos que

$$
\left.\pi_{0}\left(\alpha_{i}\right)(t)\right|_{[0,1]}=\left.\pi_{0}\left(\alpha_{i}\right)(t)\right|_{\left[0, t_{i}\right]} * \tilde{\omega}_{i}\left(\left.\pi_{0}\left(\alpha_{i}\right)(t)\right|_{\left[0, t_{i}\right]}\right) * \cdots * \tilde{\omega}_{i}^{m_{i}-1}\left(\left.\pi_{0}\left(\alpha_{i}\right)(t)\right|_{\left[0, t_{i}\right]}\right) .
$$

\section{Portanto}

$$
\begin{aligned}
\int_{0}^{1}\left|\dot{\alpha}_{i}(t)\right| d t & =\mathcal{L}\left(\left.\pi_{0}\left(\alpha_{i}\right)(t)\right|_{[0,1]}\right) \\
& =\mathcal{L}\left(\left.\left.\left.\pi_{0}\left(\alpha_{i}\right)(t)\right|_{\left[0, t_{i}\right]} * \tilde{\omega}_{i} \pi_{0}\left(\alpha_{i}\right)(t)\right|_{\left[0, t_{i}\right]} * \cdots * \tilde{\omega}_{i}^{m_{i}-1} \pi_{0}\left(\alpha_{i}\right)(t)\right|_{\left[0, t_{i}\right]}\right) \\
& \leq m_{i} \mathcal{L}\left(\left.\pi_{0}\left(\alpha_{i}\right)(t)\right|_{\left[0, t_{i}\right]}\right) \\
& =m_{i} \int_{0}^{t_{i}}\left|\dot{\alpha}_{i}(t)\right| d t \\
& =\int_{0}^{m_{i} t_{i}}\left|\dot{\alpha}_{i}(t)\right| d t .
\end{aligned}
$$

Assim

$$
t_{i} \geq \frac{1}{m_{i}} \geq \frac{1}{l}
$$

Portanto

$$
\frac{1}{l} \mathcal{L}\left(\left.\alpha_{i}\right|_{[0,1]}\right)=\frac{1}{l}\left|\omega_{i}\right| \leq t_{i}\left|\omega_{i}\right|=\mathcal{L}\left(\left.\alpha_{i}\right|_{\left[0, t_{i}\right]}\right)=: \mathcal{L}_{X}\left(\left.\alpha_{i}\right|_{[0,1]}\right) .
$$


Pela Proposição 3.6.1, a sequência real $\left\{\left|\omega_{i}\right|\right\}_{i \in \mathbb{N}}$ é não limitada. Assim, pela equação (3.6), a sequência $\left\{\mathcal{L}_{X}\left(\left.\alpha_{i}\right|_{[0,1]}\right)\right\}_{i \in \mathbb{N}}$ também é não limitada. E assim temos a segunda parte do corolário.

\subsection{Métrica da Palavra e Função Deslocamento}

Na ideia da prova do Teorema 3 obtivemos que, dada uma base $\mathcal{A}=\left\{v_{1}, \ldots, v_{k}\right\}$ de $\mathbb{Z}^{k}$ e sendo $\left\{\omega_{i}\right\}_{i \in \mathbb{N}}$ uma sequência de elementos de $\mathbb{Z}^{k}$, onde cada $\omega_{i}$ é definido da seguinte maneira

$$
\omega_{i}:=v_{1}+v_{2}+\cdots+v_{k-1}+i v_{k},
$$

então a sequência real $\left\{\left|\omega_{i}\right|\right\}_{i \in \mathbb{N}}$ é não limitada. Observemos que para cada $\omega_{i}$ temos que $\mathrm{L}_{A}\left(\omega_{i}\right)=$ $i+k-1$ (vide definição 3.7.1), e portanto, a sequência $\left\{\mathrm{L}_{A}\left(\omega_{i}\right)\right\}_{i \mathbb{N}}$ também é não limitada. Assim, surge a pergunta de se é possível encontrar uma relação entre $\mathrm{L}_{A}\left(\omega_{i}\right)$ e $\left|\omega_{i}\right|$. A resposta é afirmativa, e está dada pela seguinte proposição.

Proposição 2 (Proposição 3.7.1) Seja $X=M / \Gamma$ uma variedade Riemanniana compacta, onde $\Gamma=\mathbb{Z}^{k}$, com $k \geq 2$. Suponha que

$$
\mathcal{A}=\left\{v_{1}, v_{2}, \ldots, v_{k}\right\}
$$

é uma base para $\Gamma$. Então existem constantes positivas $r$ e $P$ tais que

$$
P \mathrm{~L}_{\mathcal{A}}(\omega) \leq \frac{1}{r}|\omega|+1 \text {, para todo } \omega \in \Gamma \text {. }
$$

Além disso, se para cada $i \geq 1$ definimos

$$
\omega_{i}:=v_{1}+v_{2}+\cdots+v_{k-1}+i v_{k}
$$

então a sequência real $\left\{\left|\omega_{i}\right|\right\}_{i \geq 1}$ é não limitada.

Observemos que no Teorema 3 não podemos garantir a existência de uma família $\left\{\left(\omega_{i},\left.\alpha_{i}\right|_{[0,1]}\right)\right\}_{i \in \mathbb{N}}$ de geodésicas fechadas em $X=M / \Gamma$ geométricamente distintas tal que a sequência $\left\{\mathcal{L}_{X}\left(\left.\alpha_{i}\right|_{[0,1]}\right)\right\}_{i \in \mathbb{N}}$ seja não limitada. O seguinte resultado mostra, da mesma maneira que no Corolário 1, que em um caso particular do grupo $\Gamma$, podemos ter tal família de geodésicas fechadas em $X$.

Proposição 3 (Proposição 3.7.2) Seja $X=M / \Gamma$ um good orbifold Riemanniano compacto, onde $M$ é uma variedade Riemanniana tal que $\operatorname{dim}(M)>1$ e $\Gamma=\tilde{\Gamma} \ltimes \mathbb{Z}^{k}$, com $k \geq 2$ e $\tilde{\Gamma}$ é um grupo 
finito. Então o orbifold Riemanniano $X=M / \Gamma$ admite uma familia $\left\{\left(\omega_{i},\left.\alpha_{i}\right|_{[0,1]}\right)\right\}_{i \in \mathbb{N}}$ de geodésicas fechadas geometricamente distintas. Além disso, se $\left\{\left(\bar{\omega}_{i},\left.\alpha_{i}\right|_{\left[0, t_{i}\right]}\right)\right\}_{i, \in \mathbb{N}}$ são geodésicas fechadas primitivas da familia $\left\{\left(\omega_{i},\left.\alpha_{i}\right|_{[0,1]}\right)\right\}_{i \in \mathbb{N}}$, então existem constantes positivas $\bar{P}$ e r tais que

$$
\bar{P} \mathrm{~L}_{\mathcal{A}}\left(\omega_{i}\right) \leq \frac{1}{r}\left|\bar{\omega}_{i}\right|+1 \text {, para todo } i \in \mathbb{N} \text {. }
$$

$e$

$$
\bar{P} \mathrm{~L}_{\mathcal{A}}\left(\omega_{i}\right) \leq \frac{1}{r} \mathcal{L}_{X}\left(\left.\alpha_{i}\right|_{[0,1]}\right)+1 \text {, para todo } i \in \mathbb{N}
$$

Em particular, $\left\{\mathcal{L}_{X}\left(\left.\alpha_{i}\right|_{[0,1]}\right)\right\}_{i \in \mathbb{N}}$ é uma sequência real não limitada (onde $\mathcal{A}$ é como na Proposição 2).

\subsection{Prova Detalhada do Teorema 1}

Antes de dar nossa primeira proposição, vamos lembrar as seguintes definições.

Definição 3.4.1 Dada uma variedade Riemanniana completa $(M, g)$, dizemos que uma geodésica não constante $\alpha: \mathbb{R} \rightarrow M$ é invariante por uma isometria $\omega$ de $(M, g)$ se existe uma constante positiva $\lambda$, tal que $\omega \alpha(t)=\alpha(t+\lambda)$, para todo $t \in \mathbb{R}$. E neste caso dizemos que $\alpha$ é $(\omega, \lambda)-$ invariante ou simplesmente $\omega$-invariante.

Observação 3.4.1 Pela definição, uma geodésica não trivial é invariante pela isometria identidade de $M$ se tal geodésica é uma geodésica periódica em $M$. Além disso, a afirmação recíproca também é válida.

Definição 3.4.2 Uma isometria $\omega$ de Iso $(M, \mathrm{~g})$ é chamada de uma translação se $\omega$ não fixa pontos de $M$ e existe uma geodésica $\gamma: \mathbb{R} \rightarrow M$ tal que $\omega(\gamma(\mathbb{R}))=\gamma(\mathbb{R})$.

O seguinte exemplo mostra uma relação que existe entre uma translação e uma isometria de tipo hiperbólico (vide [BH99], Teorema 6.8, pág. 231).

Exemplo 3.4.1 Sejam $(M, g)$ uma variedade Riemanniana de curvatura não positiva e $\omega$ um elemento Iso $(M, g)$. $\omega$ é uma translação se e somente se $\omega$ é de tipo hiperbólico.

Observação 3.4.2 Se $\omega_{1}$ é uma translação e $\gamma(t)$ é a geodésica tal que $\omega_{1}(\gamma(\mathbb{R}))=\gamma(\mathbb{R})$, é fácil ver que $\gamma$ é não trivial. Se $\alpha: \mathbb{R} \rightarrow M$ é uma geodésica invariante por uma isometria $\omega_{2}$, onde $\omega_{2}$ não fixa pontos, então $\omega_{2}$ é uma translação, 
Proposição 3.4.1 Seja $X=M / \Gamma$ um good orbifold Riemanniano. Então $\left(\omega,\left.\alpha\right|_{[0,1]}\right)$ é uma geodésica fechada em $X$ se e somente se $\alpha(t)$ é $\omega$-invariante.

Demonstração. Vamos supor que $\left(\omega,\left.\alpha\right|_{[0,1]}\right)$ é uma geodésica fechada, i.e., $\omega \alpha(0)=\alpha(1)$ e $d \omega \dot{\alpha}(0)=\dot{\alpha}(1)$. Definindo $\beta(t):=\alpha(t+1)$, para todo $t \in \mathbb{R}$, temos que $\beta(t)$ é uma geodésica. Dado que $\left(\omega,\left.\alpha\right|_{[0,1]}\right)$ é uma geodésica fechada e pela definição de $\beta(t)$, temos que $d \omega \dot{\alpha}(0)=\dot{\alpha}(1)=\dot{\beta}(0)$ e $\omega \alpha(0)=\alpha(1)=\beta(0)$. Então, dado que $\beta$ e $\omega \alpha$ são geodésicas, temos que $\omega \alpha(t)=\beta(t)=\alpha(t+1)$, para todo $t \in \mathbb{R}$. Portanto $\alpha$ é uma geodésica $\omega$-invariante.

Vamos provar a recíproca. Supomos que $\alpha$ é $(\omega, 1)$ - invariante, i.e.,

$$
\omega \alpha(t)=\alpha(t+1) \text {, para todo } t \in \mathbb{R} \text {. }
$$

Então

$$
d \omega \dot{\alpha}(t)=\dot{\alpha}(t+1) \text {, para todo } t \in \mathbb{R} \text {. }
$$

Aplicado $t=0$ em (3.7) e (3.8), obtemos que $\omega \alpha(0)=\alpha(1)$ e $d \omega \dot{\alpha}(0)=\dot{\alpha}(1)$, respectivamente. Logo $\left(\omega,\left.\alpha\right|_{[0,1]}\right)$ é uma geodésica fechada em $X$.

Proposição 3.4.2 Sejam $X=M / \Gamma$ um good orbifold Riemanniano e $\omega \in \Gamma$ uma translação em $M$, então $X$ admite uma geodésica fechada não trivial.

Demonstração. Sabemos que se $\omega$ é uma translação em $M$, então, por definição, $\omega$ não fixa pontos de $M$. Além existe uma geodésica $\alpha: \mathbb{R} \rightarrow M$, tal que $\omega(\alpha(\mathbb{R}))=\alpha(\mathbb{R})$. Dado que $\omega$ é uma isometria e $\alpha(t)$ é uma geodésica, existe $\lambda \in \mathbb{R}$ tal que $\omega \alpha(t)=\alpha( \pm t+\lambda)$, para todo $t \in \mathbb{R}$. Além disso, dado que $\omega$ não fixa pontos, temos que $\lambda \neq 0$.

Afirmamos que $\omega \alpha(t)=\alpha(t+\lambda)$, i.e., $\omega$ não muda a orientação da geodésica $\alpha(t)$. Com efeito, pois se acontece o contrário, i.e., $\omega \alpha(t)=\alpha(-t+\lambda)$, então vamos ter que $\omega^{2} \alpha(t)=\alpha(t)$, para todo $t \in \mathbb{R}$. Em especial, para $\frac{\lambda}{2}$, temos o seguinte:

$$
\alpha\left(\frac{\lambda}{2}\right)=\omega^{2} \alpha\left(\frac{\lambda}{2}\right)=\omega\left(\omega \alpha\left(\frac{\lambda}{2}\right)\right)=\omega \alpha\left(-\frac{\lambda}{2}+\lambda\right)=\omega \alpha\left(\frac{\lambda}{2}\right) .
$$

Portanto, $\omega$ fixa o ponto $\alpha\left(\frac{\lambda}{2}\right)$. Mas isso é um absurdo, pois $\omega$ é uma translação. Logo $\omega$ não muda a orientação da geodésica $\alpha(t)$.

Agora precisamos analizar os dois seguinte casos: $\lambda>0$ e $\lambda<0$. 
- Suponhamos que $\lambda>0$. Sabemos que $\omega \alpha(t)=\alpha(t+\lambda)$, para todo $t \in \mathbb{R}$. Então, pela Proposição 3.4.1, temos que $\left(\omega,\left.\alpha\right|_{[0, \lambda]}\right)$ é uma geodésica fechada em $X$.

- Se temos que $\lambda<0$, e dado que $\omega \alpha(t)=\alpha(t+\lambda)$, para todo $t \in \mathbb{R}$, temos que $\omega^{-1} \alpha(t)=$ $\alpha(t-\lambda)$, para todo $t \in \mathbb{R}$. Assim, pela Proposição 3.4.1, obtemos que $\left(\omega^{-1},\left.\alpha\right|_{[0,-\lambda]}\right)$ é uma geodésica fechada em $X$.

Observação 3.4.3 Observe que o resultado da Proposição 3.4.2 também pode ser obtido a partir do item número 2 do Teorema 2.4.1, se $\omega$ é de tipo hiperbólico. Agora, observemos que se $X$ é compacto, vamos garantir, pela Proposição 2.2.1, que toda translação em $\Gamma$ é uma isometria de tipo hiperbólico.

Exemplo 3.4.2 O seguinte resultado é conhecido em geometria Riemanniana: Seja M uma variedade riemanniana compacta não simplesmente conexa e de curvatura negativa. Seja

$$
\pi: N \longrightarrow M
$$

uma aplicação de recobrimento e considere a métrica induzida por esta aplicação em N. Então toda transformação de recobrimento

$$
\omega: N \longrightarrow N
$$

diferente da identidade é uma translação de $N$.

Assim, pela Proposição 3.4.2, temos que cada transformação de recobrimento $\omega: N \rightarrow N$ induz uma geodésica fechada em $M$.

Exemplo 3.4.3 Vamos ver dois exemplos de geodésicas fechadas a partir das Proposições 3.4.1 e 3.4.2. Sejam $M$ a esfera $\mathbb{S}^{2}$, com a métrica usual, e $\Gamma$ o grupo gerado pelas isometrias $\omega_{1}$ e $\omega_{2}$, onde $\omega_{1}$ e $\omega_{2}$ serão definidas a continuação, e $X=M / \Gamma$.

Seja $\omega_{1}$ a seguinte isometria

$$
\begin{aligned}
& \omega_{1}: \mathbb{S}^{2} \quad \longrightarrow \quad M \\
& (x, y, z) \longmapsto(x, y,-z) .
\end{aligned}
$$


E seja

$$
\begin{array}{rlcc}
\omega_{2}: & \mathbb{S}^{2} & \longrightarrow & M \\
(x, y, z) & \longmapsto & (-x,-y,-z)
\end{array}
$$

i.e., $\omega_{2}$ é a isometria antípoda de $\mathbb{S}^{2}$. Observemos que o grupo $\Gamma$ é finito, e portanto $X$ é um good orbifold Riemanniano. Seja $\alpha: \mathbb{R} \longrightarrow M$ a geodésica definida por $t \longmapsto(\cos (2 \pi t), \operatorname{sen}(2 \pi t), 0)$, i.e., $\alpha$ é a linha do Equador em $\mathbb{S}^{2}$. Observemos que $\left(\omega_{1},\left.\alpha\right|_{[0,1]}\right)$ é uma geodésica fechada em $X$, i.e., temos que $\omega_{1} \alpha(0)=\alpha(1)$ e $d \omega_{1} \dot{\alpha}(0)=\dot{\alpha}(1)$. Neste caso $\alpha$ é $\left(\omega_{1}, \lambda\right)-$ invariante, para cada $\lambda \in\{1,2,3, \ldots\}$. No entanto, $\omega_{1}$ não é uma translação, pois fixa $\alpha(t)$, para todo $t \in \mathbb{R}$.

Por outro lado, sejam p qualquer ponto de $\mathbb{S}^{2}$ e $\beta:[0,1] \rightarrow \mathbb{S}^{2}$ o segmento geodésica ligando $p$ e $q:=\omega_{2}(p)$, i.e., $\beta(0)=p$ e $\beta(1)=q$. Observemos que, dado que $\mathbb{S}^{2}$ é completa, temos que $\beta: \mathbb{R} \rightarrow \mathbb{S}^{2}$. Além disso, $\omega_{2}$ é uma translação ao longo de $\beta(t)$, pois $\omega_{2} \beta(t)=\beta(t+1)$, para todo $t \in \mathbb{R}$. Assim, pela Proposição 3.4.2, $\left(\omega,\left.\beta\right|_{[0,1]}\right)$ é uma geodésica fechada em $X$.

E fácil ver que $\omega_{1} \omega_{2}=\omega_{2} \omega_{1}$ e que $\Gamma=\left\{e, \omega_{1}, \omega_{2}, \omega_{1} \omega_{2}\right\}$, onde e é a isometria identidade de $M$.

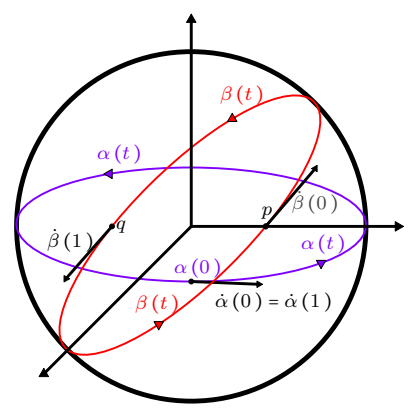

Figura 3.3: As geodésicas fechadas $\left(\omega_{1},\left.\alpha\right|_{[0,1]}\right)$ e $\left(\omega_{2},\left.\beta\right|_{[0,1]}\right)$.

Observação 3.4.4 Para aprofundar um pouco mais no tema de geodésicas invariantes por isometrias vide [Ozo69] e [Gro73].

Proposição 3.4.3 Sejam X um good orbifold Riemanniano e $\left(\omega,\left.\alpha\right|_{[0,1]}\right)$ uma geodésica fechada em X. Então

- A. Para todo $k \in \mathbb{Z}$ e para todo $t \in \mathbb{R}$, temos que $\omega^{k} \alpha(t)=\alpha(t+k)$.

- B. $\left(\omega^{-1},\left.\alpha(-t)\right|_{[0,1]}\right)$ é uma geodésica fechada em $X$.

- C. Para todo $\tilde{\omega} \in \Gamma$, temos que $\left(\tilde{\omega} \omega \tilde{\omega}^{-1},\left.\tilde{\omega} \alpha\right|_{[0,1]}\right)$ é uma geodésica fechada em $X$.

Demonstração. Primeiro vamos provar o item $A$. 
1. No caso $k=0$, temos a prova de forma imediata.

2. Vamos provar para $k \geq 1$, e vamos fazer a prova por indução.

- Se $k=1$, então temos a prova pela Proposição 3.4.1.

- Seja $k=2$. Seja $t$ qualquer número real, então

$$
\begin{aligned}
\omega^{2} \alpha(t) & =\omega(\omega \alpha(t)) \\
& =\omega \alpha(t+1) \\
& =\alpha(t+2)
\end{aligned}
$$
usando novamente a Proposição 3.4.1.

Logo vale para $k=2$.

- Vamos supor que $\omega^{k} \alpha(t)=\alpha(t+k)$, para todo $t$ em $\mathbb{R}$.

- Vamos provar para $k+1$. Seja qualquer $t \in \mathbb{R}$, então

$$
\begin{aligned}
\omega^{k+1} \alpha(t) & =\omega\left(\omega^{k} \alpha(t)\right) & & \\
& =\omega \alpha(t+k) & & \text { por hipótese de indução } \\
& =\alpha(t+k+1) & & \text { pela Proposição 3.4.1. }
\end{aligned}
$$

Portanto vale para $k+1$

Logo temos a afirmação para todo $k \geq 0$.

3. Vamos provar para $k<0$. Seja $k$ qualquer inteiro negativo mas fixo. Logo $-k>0$, e assim, pelo provado acima, temos que $\omega^{-k} \alpha(t)=\alpha(t-k)$, para todo $t \in \mathbb{R}$. Logo $\alpha(t)=$ $\omega^{k} \alpha(t-k)$, para todo $t \in \mathbb{R}$. Definamos $s:=t-k$. Assim $\alpha(s+k)=\omega^{k} \alpha(s)$. Então, para todo $s \in \mathbb{R}$, temos que $\omega^{k} \alpha(s)=\alpha(s+k)$. Logo temos que é válido para todo inteiro $k<0$.

Então temos a prova do item $A$.

Agora vamos provar o item $B$. Pelo item $A$ temos que $\omega^{-1}(t)=\alpha(t-1)$, para todo $t \in \mathbb{R}$. Assim, se definimos $\beta(t):=\alpha(-t)$, obtemos que

$$
\omega^{-1} \beta(t)=\omega^{-1} \alpha(-t)=\alpha(-t-1)=\beta(t+1), \text { para todo } t \in \mathbb{R} .
$$

Portanto, pela Proposição 3.4.1, temos que $\left(\omega^{-1},\left.\beta\right|_{[0,1]}\right)$ é uma geodésica fechada em $X$. E então temos $B$. 
Sejam $\tilde{\omega} \in \Gamma$ e $t \in \mathbb{R}$, então

$$
\begin{aligned}
\tilde{\omega} \omega \tilde{\omega}^{-1}(\tilde{\omega} \alpha(t)) & =\tilde{\omega} \omega \alpha(t) \\
& =\tilde{\omega} \alpha(t+1) \quad \text { aplicando a Proposição 3.4.1 em }\left(\omega,\left.\alpha\right|_{[0,1]}\right) .
\end{aligned}
$$

Assim, novamente pela Proposição 3.4.1, temos que $\left(\tilde{\omega} \omega \tilde{\omega}^{-1},\left.\tilde{\omega} \alpha\right|_{[0,1]}\right)$ é uma geodésica fechada em $X$. E assim temos o item $C$.

Lema 3.4.1 Seja $s \in(0,1)$. s não é da forma $\frac{1}{n}$, com $n \in\{2,3,4, \ldots\}$, se e somente se existe um único $k \in \mathbb{N}$ tal que $k s<1<(k+1) s$.

Demonstração. Vamos supor que $s \in(0,1)$ e $s$ não é da forma $\frac{1}{n}$, com $n \in\{2,3,4, \ldots\}$. Isto significa que $s>\frac{1}{2}$ ou $s<\frac{1}{2}$. No primeiro caso tomamos $k=1$, e assim temos que é válida a conclusão. Agora vamos supor que $s<\frac{1}{2}$. Pela propriedade arquimediana dos números temos que existe $l \in \mathbb{N}$ tal que $l s>1$. Observemos que tal $l$ satisfaz que $l>2$ e $l s \neq 1$, pelas hipóteses sobre $s$ Seja $l_{0}$ o mínimo número natural que satisfaz a propriedade mencionada. Definindo $k:=l_{0}-1$, temos que $k s<1<(k+1) s$.

Agora vamos provar a outra direção. Vamos supor que $s \in(0,1)$ e que existe um $k \in \mathbb{N}$ tal que $k s<1<(k+1) s$. Se não temos a conclusão, i.e., se $s=\frac{1}{n}$, $\operatorname{com} n \in\{2,3,4, \ldots\}$, então $\frac{k}{n}<1<\frac{k+1}{n}$. Assim $k<n$ e $n<k+1$, mas isso não pode acontecer. Logo temos que $s$ não é da forma $\frac{1}{n}$, com $n \in\{2,3,4, \ldots\}$.

Proposição 3.4.4 (geodésica primitiva) Vamos supor que $X=M / \Gamma$ é um good orbifold Riemanniano e que $\left(\omega,\left.\alpha\right|_{[0,1]}\right)$ é uma geodésica fechada em $X$. Então existem $t_{0} \in(0,1]$ e $\omega_{0} \in \Gamma$ tais que $\left(\omega_{0},\left.\alpha\right|_{\left[0, t_{0}\right]}\right)$ é uma geodésica fechada e se $\left(\omega_{1},\left.\alpha\right|_{\left[0, t_{1}\right]}\right)$ é outra geodésica fechada em $X$, então $t_{0} \leq t_{1}$. Além disso, $t_{0}$ é da forma $\frac{1}{n_{0}}$, onde $n_{0} \in\{1,2,3, \ldots\}$.

Demonstração. Definamos o seguinte conjunto:

$$
\Omega_{\alpha}:=\left\{t \in(0,1]: \exists \tilde{\omega} \in \Gamma \text { tal que }\left(\tilde{\omega},\left.\alpha\right|_{[0, t]}\right) \text { é uma geodésica fechada em } X\right\} \text {. }
$$

Temos que $\Omega_{\alpha}$ é não vazio pois $\left(\omega,\left.\alpha\right|_{[0,1]}\right)$ é uma geodésica fechada em $X$. Então $\Omega_{\alpha}$ possui ínfimo. Seja, então, $\tilde{t}=\inf \left(\Omega_{\alpha}\right)$. Portanto existe uma sequência $\left\{t_{i}\right\}_{i \in \mathbb{N}}$ em $\Omega_{\alpha}$ tal que $\lim _{i \rightarrow \infty} t_{i}=\tilde{t}$. Agora, dado $i \geq 1$, existe $\omega_{i} \in \Gamma$ tal que $\left(\omega_{i},\left.\alpha\right|_{\left[0, t_{i}\right]}\right)$ é uma geodésica fechada em $X$. E assim, pela Proposição 3.4.1, temos que $\omega_{i} \alpha(t)=\alpha\left(t+t_{i}\right)$, para todo $t \in \mathbb{R}$. 
Seja $\hat{t} \in \mathbb{R}$,arbitrário, e definamos $\left\{y_{i}\right\}_{i \in \mathbb{N}}:=\{\alpha(\hat{t})\}_{i \in \mathbb{N}}$, i.e., a sequência constante e igual a $\alpha(\hat{t})$. Agora,

$$
\begin{aligned}
\lim _{i \rightarrow \infty} \omega_{i} y_{i} & =\lim _{i \rightarrow \infty} \omega_{i} \alpha(\hat{t}) & & \text { dado que } y_{i}=\alpha(\hat{t}), \text { para todo } i \in \mathbb{N} \\
& =\lim _{i \rightarrow \infty} \alpha\left(\hat{t}+t_{i}\right) & & \text { pois } \omega_{i} \alpha(t)=\alpha\left(t+t_{i}\right), \text { para todo } t \in \mathbb{R} \\
& =\alpha(\hat{t}+\tilde{t}) & & \text { dado que } \lim _{i \rightarrow \infty} t_{i}=\tilde{t} .
\end{aligned}
$$

Então, dado que $\Gamma$ age propriamente sobre $M$, temos que a sequência $\left\{\omega_{i}\right\}_{i \geq 1}$ possui uma subsequência convergente. Assim, seja $\left\{\omega_{i_{k}}\right\}_{k \geq 1}$ a subsequência de $\left\{\omega_{i}\right\}_{i \geq 1}$ que é convergente, e vamos supor que $\left\{\omega_{i_{k}}\right\}_{k \geq 1}$ converge a $\omega_{0} \in \Gamma$. Como $\Gamma$ é um grupo discreto, temos que a subsequência é constante, salvo por um número finito de índices. Então podemos supor que a subsequência $\left\{\omega_{i_{k}}\right\}_{k \geq 1}$ é constante e igual a $\omega_{0} \in \Gamma$. Logo, como nós provamos que para todo $\hat{t} \in \mathbb{R}$ acontece que $\lim _{i \rightarrow \infty} \omega_{i} \alpha(\hat{t})=\alpha(\hat{t}+\tilde{t})$, em particular $\lim _{k \rightarrow \infty} \omega_{i_{k}} \alpha(\hat{t})=\alpha(\hat{t}+\tilde{t})$, e que $\omega_{i_{k}}=\omega_{0}$, para todo $k \geq 1$, então $\omega_{0} \alpha(\hat{t})=\alpha(\hat{t}+\tilde{t})$, para todo $\hat{t} \in \mathbb{R}$. Assim, pela Proposição 3.4 .1 e definindo $t_{0}:=\tilde{t}$, temos que $\left(\omega_{0},\left.\alpha\right|_{\left[0, t_{0}\right]}\right)$, é uma geodésica fechada em $X$.

Afirmamos que $t_{0}>0$. Vamos supor que $t_{0}=0$. Assim, para todo $t \in \mathbb{R}$, temos que

$$
\omega_{0} \alpha(t)=\alpha\left(t+t_{0}\right)=\alpha(t) .
$$

Dado que $\omega_{i_{k}}=\omega_{0}$, para todo $k \geq 1$, e que $\omega_{i_{k}} \alpha(t)=\alpha\left(t+t_{i_{k}}\right)$, então temos que

$$
\alpha(t)=\omega_{0} \alpha(t)=\omega_{i_{k}} \alpha(t)=\alpha\left(t+t_{i_{k}}\right) \text {, para todo } t \in \mathbb{R} .
$$

Dado que $t_{i_{k}}>0$, para todo $k \geq 1$, então temos que $\alpha: \mathbb{R} \rightarrow M$ é uma geodésica periódica em $M$. Se definimos $s_{0}>0$ como o período de $\alpha(t)$, então $s_{0}$ divide cada $t_{i_{k}}$, em particular $s_{0} \leq t_{i_{k}}$, para cada $k \geq 1$. Mas, sabemos que $\lim _{k \rightarrow \infty} t_{i_{k}}=t_{0}$, portanto $s_{0} \leq t_{0}$, que é um absurdo, pois estamos supondo que $t_{0}=0$. Logo $t_{0}>0$, é o elemento mínimo de $\Omega_{\alpha}$.

Agora queremos provar que $t_{0}$ é da forma $\frac{1}{n_{0}}$, onde $n_{0} \in\{1,2,3, \ldots\}$. Se $t_{0}=1$, então temos o resultado. Vamos supor então que $t_{0} \in(0,1)$ e que $t_{0} \neq \frac{1}{n_{0}}$, para todo $n_{0} \in\{2,3,4, \ldots\}$. Assim, pelo Lema 3.4.1, existe um único $k \in \mathbb{N}$ tal que $k t_{0}<1<(k+1) t_{0}$. Observemos que para todo $t \in \mathbb{R}$, 
temos que

$$
\begin{aligned}
\omega \omega_{0}^{-k} \alpha(t) & =\omega \alpha\left(t-k t_{0}\right) & & \text { aplicando a Proposição 3.4.3 em }\left(\omega_{0},\left.\alpha\right|_{\left[0, t_{0}\right]}\right) \\
& =\alpha\left(t-k t_{0}+1\right) & & \text { aplicando a Proposição 3.4.1 em }\left(\omega,\left.\alpha\right|_{[0,1]}\right) .
\end{aligned}
$$

Logo, pela Proposição 3.4.1, $\left(\omega \omega_{0}^{-k},\left.\alpha\right|_{\left[0,1-k t_{0}\right]}\right)$ é uma geodésica fechada. Dado que $t_{0}$ é o mínimo de $\Omega_{\alpha}$, obtemos que $t_{0} \leq 1-k t_{0}$, que é um absurdo, pois $k t_{0}<1<(k+1) t_{0}$. Então $t_{0}$ é da forma desejada.

Definição 3.4.3 Dados um good orbifold Riemanniano $X$ e $\left(\omega,\left.\alpha\right|_{[0,1]}\right)$ uma geodésica fechada em $X$, definimos uma geodésica fechada primitiva associada à geodésica fechada $\left(\omega,\left.\alpha\right|_{[0,1]}\right)$ como $\left(\omega_{0},\left.\alpha\right|_{\left[0, t_{0}\right]}\right)$, onde $t_{0}$ e $\omega_{0}$ são dados pela Proposição 3.4.4.

Observação 3.4.5 Na definição anterior não podemos falar de uma única geodésica fechada primitiva associada à geodésica fechada $\left(\omega,\left.\alpha\right|_{[0,1]}\right)$, dado que pode acontecer que $\omega_{0}$ não seja único. É fácil ver que $\omega_{0}$ é único se existe algum $\bar{t}$ em $\mathbb{R}$ tal que $\Gamma_{\alpha(\bar{t})}$ somente contém o elemento identidade de $\Gamma$, i.e., $\Gamma_{\alpha(\bar{t})}$ é trivial. E isto pode ser visto no seguinte resultado.

Proposição 3.4.5 Sejam $X=M / \Gamma$ um good orbifold Riemanniano e $\left(\omega,\left.\alpha\right|_{[0,1]}\right)$ uma geodésica fechada em X. Então temos as seguintes propriedades:

1. Se $\left(\omega_{0},\left.\alpha\right|_{\left[0, t_{0}\right]}\right)$ é uma geodésica fechada primitiva associada à geodésica fechada $\left(\omega,\left.\alpha\right|_{[0,1]}\right)$, então existem $n, m \in \mathbb{N}$ tais que $\omega_{0}^{n}=\omega^{m}$.

2. Se $\left(\omega_{1},\left.\alpha\right|_{\left[0, t_{1}\right]}\right)$ é uma geodésica fechada, então $t_{1}=k t_{0}$, para algum $k \in \mathbb{N}$;

3. se $\left(\hat{\omega}_{0},\left.\alpha\right|_{\left[0, t_{0}\right]}\right)$ é outra geodésica fechada primitiva associada à geodésica fechada $\left(\omega,\left.\alpha\right|_{[0,1]}\right)$ e existe $\bar{t} \in \mathbb{R}$ tal que $\alpha(\bar{t})$ é ponto regular, então $\hat{\omega}_{0}$ e $\omega_{0}$ são iguais.

\section{Demonstração.}

1. Seja $\left(\omega_{0},\left.\alpha\right|_{\left[0, t_{0}\right]}\right)$ uma geodésica primitiva associada à geodésica fechada $\left(\omega,\left.\alpha\right|_{[0,1]}\right)$. Logo, pela Proposição 3.4.4, temos que $t_{0}=\frac{1}{n_{0}}$, onde $n_{0} \in\{1,2,3, \ldots\}$. Seja $k$ qualquer número natural, mas fixo, então

$$
\begin{aligned}
\omega_{0}^{n_{0} k} \alpha(0) & =\alpha(k) \\
& =\omega^{k} \alpha(0)
\end{aligned}
$$

aplicando a Proposição $3.4 .3 \mathrm{em}\left(\omega_{0},\left.\alpha\right|_{\left[0, t_{0}\right]}\right)$ aplicando a Proposição 3.4.3 em $\left(\omega,\left.\alpha\right|_{[0,1]}\right)$. 
Assim, $\omega^{-k} \omega_{0}^{n_{0} k}$ é um elemento de $\Gamma_{\alpha(0)}$, para cada $k \in \mathbb{N}$. Dado que $\Gamma_{\alpha(0)}$ é finito, existem $k_{1}$ e $k_{2} \in \mathbb{N}$ tais que $\omega^{-k_{1}} \omega_{0}^{n_{0} k_{1}}=\omega^{-k_{2}} \omega_{0}^{n_{0} k_{2}}$. Supondo $k_{1}<k_{2}$, definindo $n:=n_{0}\left(k_{2}-k_{1}\right)$ e $m:=k_{2}-k_{1}$, obtemos o desejado.

2. Sabemos que $t_{0} \leq t_{1}$. Vamos supor que $t_{1}$ não é da forma $k t_{0}$, para todo $k \in\{1,2,3, \ldots\}$. Então, pelo Lema 3.4.1, existe único $l \in \mathbb{N}$ tal que $l t_{0}<t_{1}<(l+1) t_{0}$. Agora, para todo $t \in \mathbb{R}$, temos que

$$
\begin{aligned}
\omega_{1}^{-1} \omega_{0}^{l+1} \alpha(t) & =\omega_{1}^{-1} \alpha\left(t+(l+1) t_{0}\right) & & \text { pela Proposição 3.4.3 em }\left(\omega_{0},\left.\alpha\right|_{\left[0, t_{0}\right]}\right) \\
& =\alpha\left(t+(l+1) t_{0}-t_{1}\right) & & \text { aplicando a Proposição 3.4.3 em }\left(\omega_{1},\left.\alpha\right|_{\left[0, t_{1}\right]}\right) .
\end{aligned}
$$

Logo, pela Proposição 3.4.1, obtemos que $\left(\omega_{1}^{-1} \omega_{0}^{l+1},\left.\alpha\right|_{\left[0,(l+1) t_{0}-t_{1}\right]}\right)$ é uma geodésica fechada. Observemos que $0<(l+1) t_{0}-t_{1}<t_{0}$, dado que $l t_{0}<t_{1}<(l+1) t_{0}$. Mas é absurdo que $\left(\omega_{1}^{-1} \omega_{0}^{l+1},\left.\alpha\right|_{\left[0,(l+1) t_{0}-t_{1}\right]}\right)$ seja uma geodésica fechada, pois $\left(\omega_{0},\left.\alpha\right|_{\left[0, t_{0}\right]}\right)$ é uma geodésica fechada primitiva. Então $t_{1}$ é da forma $k t_{0}$, para algúm $k \in \mathbb{N}$.

3. Vamos supor que $\left(\hat{\omega}_{0},\left.\alpha\right|_{\left[0, t_{0}\right]}\right)$ é outra geodésica fechada primitiva. Agora, aplicando a Proposição 3.4 .3 em $\left(\hat{\omega}_{0},\left.\alpha\right|_{\left[0, t_{0}\right]}\right)$ e $\left(\omega_{0},\left.\alpha\right|_{\left[0, t_{0}\right]}\right)$, obtemos que $\hat{\omega}_{0} \alpha(\bar{t})=\alpha\left(\bar{t}+t_{0}\right)=$ $\omega_{0} \alpha(\bar{t})$. Então $\omega_{0}^{-1} \hat{\omega}_{0} \in \Gamma_{\alpha(\bar{t})}$. Assim $\hat{\omega}_{0}=\omega_{0}$, dado que $\Gamma_{\alpha(\bar{t})}$ é trivial.

Observação 3.4.6 Sejam X um good orbifold Riemanniano e $\left(\omega,\left.\alpha\right|_{[0,1]}\right)$ uma geodésica fechada em X. Supomos que $\left(\omega_{0},\left.\alpha\right|_{\left[0, t_{0}\right]}\right)$ é uma geodésica primitiva associada à geodésica $\left(\omega,\left.\alpha\right|_{[0,1]}\right)$. Então observemos que

1. O conjunto

$$
\Omega_{\alpha}=\left\{t \in\left[t_{0}, 1\right] \mid \exists \tilde{\omega} \in \Gamma \text { tal que }\left(\tilde{\omega},\left.\alpha\right|_{[0, t]}\right) \text { é uma geodésica fechada em } X\right\} .
$$

é discreto;

2. $\omega_{0}$ é de ordem finita se e somente se $\omega$ é de ordem finita.

O item número 1 segue-se do fato que se $t \in \Omega_{\alpha}$ então, pela Proposição 3.4.4, $t=k t_{0}$, onde $k \in\{1,2, \ldots\}$. E o item número 2 é obtido do item número 1 da Proposição 3.4.5.

Proposição 3.4.6 Sejam $X=M / \Gamma$ um good orbifold Riemanniano e $\left(\omega,\left.\alpha\right|_{[0,1]}\right)$ uma geodésica fechada em $X$. Então $\omega$ tem ordem finita se e somente se a é uma geodésica periódica em $M$. 
Demonstração. Primeiro vamos supor que $\omega$ tem ordem finita, i.e., existe $k \in \mathbb{N}$ tal que $\omega^{k}=$ $e$. Dado que $\left(\omega,\left.\alpha\right|_{[0,1]}\right)$ é uma geodésica fechada em $X$, temos que para todo $n \in \mathbb{N}$ a curva $\omega^{n} \alpha(t) * \omega^{n-1} \alpha(t) * \cdots * \omega \alpha(t) * \alpha(t)$, para $t \in[0,1]$, é a geodésica $\alpha:[0, n] \rightarrow M$. Dado que $\omega^{k}=e$ e pela Proposição 3.4.3, temos que $\alpha(k)=\omega^{k} \alpha(0)=\alpha(0)$. Logo a geodésica

$$
\alpha(t) * \omega \alpha(t) * \cdots * \omega^{k-2} \alpha(t) * \omega^{k-1} \alpha(t)
$$

i.e., a concatenação dos segmentos de geodésica $\alpha(t), \omega \alpha(t), \ldots, \omega^{k-1} \alpha(t)$ e $\omega^{k} \alpha(t)$, para $t \epsilon$ [0,1], é uma geodésica periódica em $M$ (ver figura 3.4).

Vamos provar a recíproca. Suponha que $\alpha$ é uma geodésica fechada não trivial em $M$. Então temos que existe um $t_{1}>0$ tal que $\alpha:\left[0, t_{1}\right] \rightarrow M$ é uma geodésica periódica em $M$, i.e., vamos supor que $t_{1}$ é o período da geodésica $\alpha(t)$, portanto $\left(e,\left.\alpha\right|_{\left[0, t_{1}\right]}\right)$ é uma geodésica fechada em $X$. Seja $\left(\omega_{0},\left.\alpha\right|_{\left[0, t_{0}\right]}\right)$ uma geodésica fechada primitiva associada à geodésica $\left(\omega,\left.\alpha\right|_{[0,1]}\right)$, então, pelo item 1 da Proposição 3.4.5, temos que existem $m, n \in \mathbb{N}$ tais que $\omega_{0}^{n}=\omega^{m}$. Agora, também temos que $\left(\omega_{0},\left.\alpha\right|_{\left[0, t_{0}\right]}\right)$ é uma geodésica primitiva associada à geodésica $\left(e,\left.\alpha\right|_{\left[0, t_{1}\right]}\right)$, logo, novamente pelo item 1 da Proposição 3.4.5, temos que existe $k \in \mathbb{N}$ tal que $\omega_{0}^{k}=e$. Portanto $e=\omega_{0}^{n k}=\omega^{m k}$, e assim temos que $\omega$ tem ordem finita.

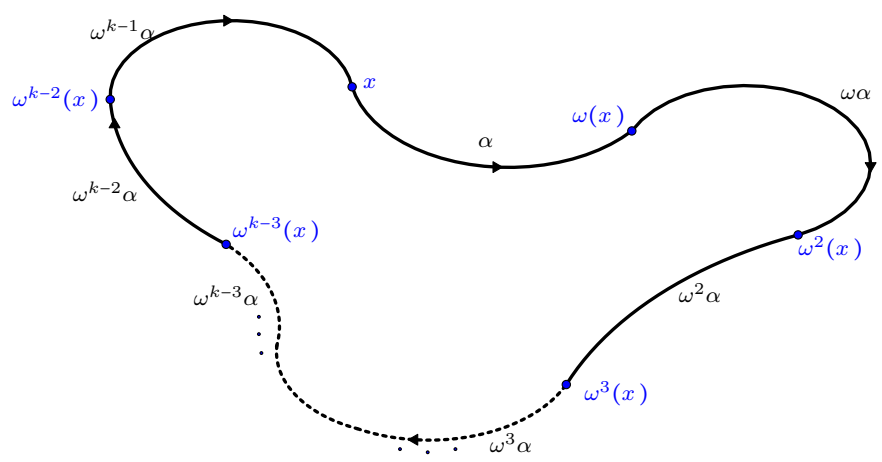

Figura 3.4: A curva $\alpha(t) * \omega \alpha(t) * \cdots * \omega^{k-1} \alpha(t) * \omega^{k} \alpha(t)$ é uma geodésica perdiódica em $M$.

Antes de dar o seguinte resultado, o qual vai ser fundamental para obter os principais resultados do capítulo, vamos lembrar duas definições.

Definição 3.4.4 Dado um espaço topológico $Y$, dizemos que ele é um espaço de Baire se dada uma familia contável de fechados $\left\{A_{i}\right\}_{i \in \mathbb{N}}$ de $Y$, tal que cada $A_{i}$ tem interior vazio, então $\bigcup_{i \in \mathbb{N}} A_{i}$ também é um conjunto com interior vazio. 
Definição 3.4.5 Dado um good orbifold Riemanniano $X=M / \Gamma$, dizemos que duas geodésicas fechadas $\left(\omega_{1},\left.\alpha_{1}\right|_{[0,1]}\right)$ e $\left(\omega_{2},\left.\alpha_{2}\right|_{[0,1]}\right)$ são geometricamente iguais ou geometricamente equivalentes em $X$ se e somente se

$$
\operatorname{imagem}\left(\pi\left(\alpha_{1}\right)\right)=\operatorname{imagem}\left(\pi\left(\alpha_{2}\right)\right) \text {. }
$$

Onde $\pi: M \longrightarrow M / \Gamma$ é a aplicação quociente.

Proposição 3.4.7 Sejam $X=M / \Gamma$ um good orbifold Riemanniano, $\left(\omega_{1},\left.\alpha_{1}\right|_{[0,1]}\right)$ e $\left(\omega_{2},\left.\alpha_{2}\right|_{[0,1]}\right)$ duas geodésicas fechadas em $X$. Então $\left(\omega_{1},\left.\alpha_{1}\right|_{[0,1]}\right)$ e $\left(\omega_{2},\left.\alpha_{2}\right|_{[0,1]}\right)$ são geometricamente iguais em $X$ se, e somente se, existem $\omega \in \Gamma$ e números reais a $e b$, tais que

$$
\omega \alpha_{1}(a t+b)=\alpha_{2}(t), \text { para todo } t \in \mathbb{R}
$$

Em particular, para todo $\tilde{\omega} \in \Gamma$, temos que as geodésicas fechadas $\left(\tilde{\omega} \omega_{1} \tilde{\omega}^{-1},\left.\tilde{\omega} \alpha_{1}\right|_{[0,1]}\right)$ e $\left(\omega_{1},\left.\alpha_{1}\right|_{[0,1]}\right)$ são geometricamente iguais em $X$.

Observação 3.4.7 Seja X um good orbifold. Observemos que a relação "geometricamente iguais" entre geodésicas fechadas em $X$ define una relação de equivalência.

Demonstração. Primeiro vamos supor que $\left(\omega_{1},\left.\alpha_{1}\right|_{[0,1]}\right)$ e $\left(\omega_{2},\left.\alpha_{2}\right|_{[0,1]}\right)$ são geométricamente iguais em $X$. Dado que $M$ é uma variedade Riemanniana completa, temos que as geodésicas $\alpha_{1}(t)$ e $\alpha_{2}(t)$ estão definidas em todo $\mathbb{R}$. Seja $s_{0}$ um número real, e vamos supor que $U$ é uma carta orbifold de $\alpha_{1}\left(s_{0}\right)$, e podemos supor que $U$ é uma bola totalmente normal para $\alpha_{1}\left(s_{0}\right)$. Seja $\epsilon>0$, e definamos $I_{\epsilon}:=\left[s_{0}-\epsilon, s_{0}+\epsilon\right]$ tal que $\left.\alpha_{1}(t)\right|_{I_{\epsilon}} \subseteq U$. Sabemos, por hipótese, que $\pi \circ \alpha_{1}(\mathbb{R})=$ $\pi \circ \alpha_{2}(\mathbb{R})$, então $\pi \circ \alpha_{1}\left(I_{\epsilon}\right) \subseteq \pi \circ \alpha_{2}(\mathbb{R})$. Assim

$$
\left.\pi \circ \alpha_{1}(t)\right|_{I_{\epsilon}} \subseteq \bigcup_{j \in \Theta} \pi \circ \alpha_{2}\left(I_{\delta_{j}}\right)
$$

onde $\Theta$ pode ser escolhido como um conjunto enumerável e tal que cada $I_{\delta_{i}}$ seja um intervalo fechado em $\mathbb{R}$. Logo

$$
\alpha_{1}\left(I_{\epsilon}\right)=U \cap \alpha_{1}\left(I_{\epsilon}\right) \subseteq\left[\bigcup_{j \in \Theta, \omega_{l} \in \Gamma} \omega_{l} \alpha_{2}\left(I_{\delta_{j}}\right)\right] \cap U=\bigcup_{j \in \Theta, \omega_{l} \in \Gamma}\left[\omega_{l} \alpha_{2}\left(I_{\delta_{j}}\right) \cap U\right]
$$

onde a união é enumerável, pois $\Gamma$ e $\Theta$ são enumeráveis. Agora, podemos escolher cada $\delta_{j}$ suficientemente pequeno tal que $\alpha_{2}\left(I_{\delta_{j}}\right)$ está contido em $U$ e $\left.\alpha_{2}(t)\right|_{I_{\delta_{j}}}: I_{\delta_{j}} \rightarrow M$ seja um mergulho. 
Logo

$$
\alpha_{1}\left(I_{\epsilon}\right)=\bigcup_{j \in \Theta, \omega_{l} \in \Gamma}\left[\omega_{l} \alpha_{2}\left(I_{\delta_{j}}\right) \cap \alpha_{1}\left(I_{\epsilon}\right)\right] .
$$

Agora, sabemos que $\alpha_{1}\left(I_{\epsilon}\right)$ é um espaço métrico completo e, por [Mun00] (Teorema 48.2), então $\alpha_{1}\left(I_{\epsilon}\right)$ é um espaço de Baire. Também sabemos que para cada $\omega_{l} \in \Gamma$ e cada $j \in \Theta$, o conjunto $\omega_{l} \alpha_{2}\left(I_{\delta_{j}}\right) \cap \alpha_{1}\left(I_{\epsilon}\right)$ é fechado em $\alpha_{1}\left(I_{\epsilon}\right)$. Logo, dado que $\alpha_{1}\left(I_{\epsilon}\right)$ é um espaço de Baire, temos que existem $j_{0} \in \Theta$ e $\omega_{l_{0}} \in \Gamma$ tais que o interior de $\left[\omega_{l_{0}} \alpha_{2}\left(I_{\delta_{j_{0}}}\right) \cap \alpha_{1}\left(I_{\epsilon}\right)\right]$, como subespaço de $\alpha_{1}\left(I_{\epsilon}\right)$, é não vazio. Assim, seja $q$ um ponto em $\omega_{l_{0}} \alpha_{2}\left(I_{\delta_{j_{0}}}\right) \cap \alpha_{1}\left(I_{\epsilon}\right)$, então existe $r>0$ tal que

$$
B_{r}(q) \cap \alpha_{1}\left(I_{\epsilon}\right) \subset \omega_{l_{0}} \alpha_{2}\left(I_{\delta_{j_{0}}}\right) \cap \alpha_{1}\left(I_{\epsilon}\right)
$$

Logo existem $s_{1}$ em $I_{\epsilon}$ e $\delta_{1}>0$ tais que

$$
\alpha_{1}\left(\left[s_{1}-\delta_{1}, s_{1}+\delta_{1}\right]\right) \subseteq \omega_{l_{0}} \alpha_{2}\left(I_{\delta_{j_{0}}}\right) .
$$

Denotaremos, por comodidade, o intervalo $\left[s_{1}-\delta_{1}, s_{1}+\delta_{1}\right]$ por $I_{\delta_{1}}$ e às geodésicas $\left.\omega_{l_{0}} \alpha_{2}(t)\right|_{\delta_{j_{0}}}$ por $\bar{\alpha}_{2}(t)$ e $\left.\alpha_{1}(t)\right|_{I_{\delta_{1}}}$ por $\bar{\alpha}_{1}(t)$. Temos que $\bar{\alpha}_{1}: I_{\delta_{1}} \rightarrow M$ e $\alpha_{1}: I_{\delta_{j_{0}}} \rightarrow M$ são mergulhos e $\bar{\alpha}_{2}\left(I_{\delta_{1}}\right) \subseteq \alpha_{1}\left(I_{\epsilon}\right)$. Agora, por [War83] (Teorema 1.32), existe uma única aplicação suave $\Phi: I_{\delta_{1}} \rightarrow I_{\delta_{j_{0}}}$ tal que $\bar{\alpha}_{1}(t)=\bar{\alpha}_{2} \circ \Phi(t)$, para todo $t \in I_{\delta_{1}}$. Agora, $\Phi$ é injetora, pois $\bar{\alpha}_{2}$ e $\left.\bar{\alpha}_{1}\right|_{I_{\epsilon}}$, são mergulhos. Logo $\Phi$ é um difeomorfismo sobre sua imagem. Dado que $\bar{\alpha}_{1}(t)=\bar{\alpha}_{2} \circ \Phi(t)$, para todo $t \in I_{\delta_{1}}$, e que $\bar{\alpha}_{2}(t)$ e $\bar{\alpha}_{1}(t)$ são geodésicas, temos que existem $\bar{a}, \bar{b} \in \mathbb{R}$, onde $\bar{a} \neq 0$, pois $\Phi$ é um difeomorfismo, tais que $\Phi(t)=\bar{a} t+\bar{b}$, para todo $t \in I_{\delta_{1}}$. Dado que $\bar{\alpha}_{1}(t)=\bar{\alpha}_{2}(\bar{a} t+\bar{b})$, para todo $t \in I_{\delta_{1}}$, e que $\bar{\alpha}_{1}$ e $\bar{\alpha}_{2}$ são geodésicas, temos que $\bar{\alpha}_{1}(t)=\bar{\alpha}_{2}(\bar{a} t+\bar{b})$, para todo $t \in \mathbb{R}$. Definindo $a=\frac{1}{\bar{a}}, b=-\frac{\bar{b}}{\bar{a}}$ e $\omega=\omega_{l_{0}}^{-1}$, temos que $\omega \alpha_{1}(a t+b)=\alpha_{2}(t)$, para todo $t \in \mathbb{R}$. Logo temos a primeira parte da proposição.

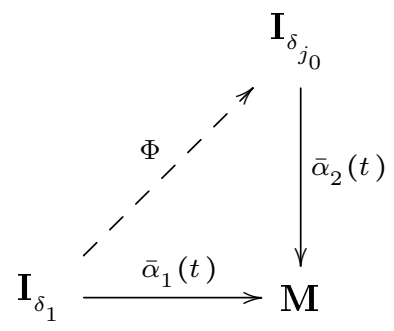

Agora vamos provar a recíproca. Vamos supor que existem $\omega \in \Gamma$ e números reais $a$ e $b$, tais que $\omega \alpha_{1}(a t+b)=\alpha_{2}(t)$, para todo $t \in \mathbb{R}$. Portanto temos que imagem $\left(\pi \circ \alpha_{1}\right)=\operatorname{imagem}\left(\pi \circ \alpha_{2}\right)$, e assim a recíproca é válida. 
Observação 3.4.8 Na prova anterior não foi usada a hipótese que $\left(\omega_{1},\left.\alpha_{1}\right|_{[0,1]}\right)$ e $\left(\omega_{2},\left.\alpha_{2}\right|_{[0,1]}\right)$ são geodésicas fechadas; somente usamos o fato que $\alpha_{i}$, para $i=1,2$, são geodésicas em $M$ e que $M$ é uma variedade Riemanniana completa. O seguinte exemplo dá uma ideia de como a Proposição 3.4.7 pode falhar quando as curvas não são geodésicas.

Exemplo 3.4.4 Sejam $M=\mathbb{R}^{2}$, com a métrica Riemanniana usual, e $\Gamma$ o grupo gerado pela seguinte isometria de $M$

$$
\begin{aligned}
& R_{y}: \quad \mathbb{R}^{2} \quad \longrightarrow \quad \mathbb{R}^{2} \\
& (p, q) \longmapsto(p,-q) \text {. }
\end{aligned}
$$

Temos então que $\Gamma=\left\{R_{x}, e\right\}$ é um subgrupo finito de isometrias de $M$, onde e é a isometria identidade de $M$, e $M / \Gamma$ é um good orbifold Riemanniano.

Sejam $\beta_{1}, \beta_{2}: \mathbb{R} \rightarrow M$, definidas por $\beta_{1}(t)=\left(t, t^{3}\right)$ e $\beta_{2}(t)=\left(t,\left|t^{3}\right|\right)$. Temos que $\beta_{1}$ e $\beta_{2}$ são curvas de classe $C^{2}$ e imagem $\left(\pi \circ \beta_{1}\right)=\operatorname{imagem}\left(\pi \circ \beta_{2}\right)$, mais ainda, $\pi \circ \beta_{1}(t)=\pi \circ \beta_{2}(t)$, para todo $t \in \mathbb{R}$. Não obstante, não existem $a, b \in \mathbb{R}$ e $\omega \in \Gamma$ tais que $\omega \beta_{1}(a t+b)=\beta_{2}(t)$, para todo $t \in \mathbb{R}$.

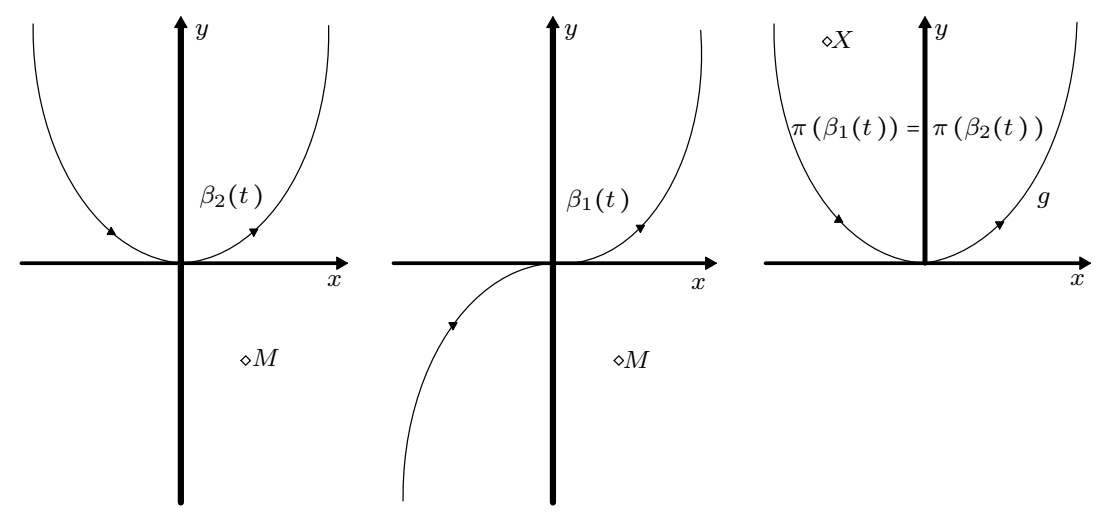

Figura 3.5: $\beta_{2}(t)$ em $M, \beta_{1}(t)$ em $M$ e $\pi\left(\beta_{1}(t)\right)=\pi\left(\beta_{2}(t)\right)$ em $X$

Proposição 3.4.8 Sejam $X=M / \Gamma$ um good orbifold Riemanniano e $\left(\omega_{1},\left.\alpha_{1}\right|_{[0,1]}\right)$ e $\left(\omega_{2},\left.\alpha_{2}\right|_{[0,1]}\right)$ duas geodésicas fechadas em $X$. Se elas são geometricamente iguais e $\left(\bar{\omega}_{1},\left.\alpha_{1}\right|_{\left[0, t_{1}\right]}\right)$ e $\left(\bar{\omega}_{2},\left.\alpha_{2}\right|_{\left[0, t_{2}\right]}\right)$ são geodésicas fechadas primitivas de $\left(\omega_{1},\left.\alpha_{1}\right|_{[0,1]}\right)$ e $\left(\omega_{2},\left.\alpha_{2}\right|_{[0,1]}\right)$, respectivamente, então o número real a, dado pela Proposição 3.4.7, que satisfaz

$$
\omega \alpha_{1}(a t+b)=\alpha_{2}(t), \text { para todo } t \in \mathbb{R}
$$

cumpre que $|a|=\frac{t_{1}}{t_{2}}$. 
Demonstração. Dado que $\left(\omega_{1},\left.\alpha_{1}\right|_{[0,1]}\right)$ e $\left(\omega_{2},\left.\alpha_{2}\right|_{[0,1]}\right)$ são geodésicas fechadas com a mesma imagem em $X$, então, pela Proposição 3.4.7, existem $\omega$ em $\Gamma$ e números reais $a, b$, com $a \neq 0$, tais que $\omega \alpha_{1}(a t+b)=\alpha_{2}(t)$, para todo $t \in \mathbb{R}$. Agora, para cada $s \in \mathbb{R}$, temos

$$
\begin{aligned}
\bar{\omega}_{2} \omega \alpha_{1}(s) & =\bar{\omega}_{2} \alpha_{2}\left(\frac{s-b}{a}\right) & & \text { dado que } \omega \alpha_{1}(a t+b)=\alpha_{2}(t), \text { para todo } t \in \mathbb{R} \\
& =\alpha_{2}\left(\frac{s-b}{a}+t_{2}\right) & & \text { aplicando a Proposição } 3.4 .1 \text { em }\left(\bar{\omega}_{2},\left.\alpha_{2}\right|_{\left[0, t_{2}\right]}\right) \\
& =\omega \alpha_{1}\left(s+a t_{2}\right) & & \text { pois } \omega \alpha_{1}(a t+b)=\alpha_{2}(t), \text { para todo } t \in \mathbb{R} .
\end{aligned}
$$

Dado que $a \neq 0$, então temos os dois possíveis casos para provar a afirmação: $a>0$ e $a<0$.

1. Supomos que $a>0$. Temos, pela equação (3.9) e pela Proposição 3.4.1, que $\left(\bar{\omega}_{2},\left.\omega \alpha_{1}\right|_{\left[0, a t_{2}\right]}\right)$ é uma geodésica fechada. Portanto, pela Proposição 3.4.3, obtemos que $\left(\omega^{-1} \bar{\omega}_{2} \omega,\left.\alpha_{1}\right|_{\left[0, a t_{2}\right]}\right)$ é uma geodésica fechada em $X$. Logo, dado que $\left(\bar{\omega}_{1},\left.\alpha_{1}\right|_{\left[0, t_{1}\right]}\right)$ é geodésica primitiva e pelo item número 2 da Proposição 3.4.5, existe $k \in\{1,2,3, \ldots\}$ tal que $k t_{1}=a t_{2}$. Assim $a=\frac{k t_{1}}{t_{2}}$. Queremos provar que $k$ é igual a 1, mas vamos supor que isso não acontece, i.e., vamos supor que $k \geq 2$.

Seja $s \in \mathbb{R}$, então temos

$$
\begin{aligned}
\omega \bar{\omega}_{1} \omega^{-1} \alpha_{2}(s) & =\omega \bar{\omega}_{1} \alpha_{1}(a s+b) & & \text { dado que } \omega \alpha_{1}(a t+b)=\alpha_{2}(t) \\
& =\omega \alpha_{1}\left(a s+b+t_{1}\right) & & \text { pois }\left(\bar{\omega}_{1},\left.\alpha_{1}\right|_{\left[0, t_{1}\right]}\right) \text { é uma geodésica fechada } \\
& =\omega \alpha_{1}\left(a\left(s+\frac{t_{1}}{a}\right)+b\right) & & \\
& =\alpha_{2}\left(s+\frac{t_{1}}{a}\right) & & \text { dado que } \omega \alpha_{1}(a t+b)=\alpha_{2}(t) \\
& =\alpha_{2}\left(s+\frac{t_{2}}{k}\right) & & \text { pois } a=\frac{k t_{1}}{t_{2}} .
\end{aligned}
$$

Isto implica, pela Proposição 3.4.1, que $\left(\omega \bar{\omega}_{1} \omega^{-1},\left.\alpha_{2}\right|_{\left[0, \frac{t_{2}}{k}\right]}\right)$ é uma geodésica fechada, mas isso é um absurdo, pois $\frac{t_{2}}{k}<t_{2}$, dado que $k>2$, e $\left(\bar{\omega}_{2},\left.\alpha_{2}\right|_{\left[0, t_{2}\right]}\right)$ é geodésica primitiva. Então $k=1$, e assim $a=\frac{t_{1}}{t_{2}}$.

2. Vamos supor que $a<0$. Provamos na equação (3.9) que $\bar{\omega}_{2} \omega \alpha_{1}(s)=\omega \alpha_{1}\left(s+a t_{2}\right)$, para todo $s \in \mathbb{R}$. Seja $s \in \mathbb{R}$ fixo, mas qualquer. Então $\bar{\omega}_{2} \omega \alpha_{1}(-s)=\omega \alpha_{1}\left(-s+a t_{2}\right)$. Logo $\omega \alpha_{1}(-s)=$ $\bar{\omega}_{2}^{-1} \omega \alpha_{1}\left(-s+a t_{2}\right)$. Definindo $t=-s+a t_{2}$, obtemos que $\omega \alpha_{1}\left(t-a t_{2}\right)=\bar{\omega}_{2}^{-1} \omega \alpha(t)$. Dado que $s$ é qualquer número real e que $t=-s+a t_{2}$, temos que $\omega \alpha_{1}\left(t-a t_{2}\right)=\bar{\omega}_{2}^{-1} \omega \alpha(t)$, para todo $t \in \mathbb{R}$. Logo, pela Proposição 3.4.1, $\left(\bar{\omega}_{2}^{-1},\left.\omega \alpha_{1}\right|_{\left[0,-a t_{2}\right]}\right)$ é uma geodésica fechada. E assim, pela Proposição 3.4.3, obtemos que $\left(\omega^{-1} \bar{\omega}_{2}^{-1} \omega,\left.\alpha_{1}\right|_{\left[0,-a t_{2}\right]}\right)$ é uma geodésica fechada em $X$. 
Agora, dado que $\left(\bar{\omega}_{1},\left.\alpha_{1}\right|_{\left[0, t_{1}\right]}\right)$ é geodésica primitiva e pelo item número 2 da Proposição 3.4 .5 , existe $k \in\{1,2,3, \ldots\}$ tal que $k t_{1}=-a t_{2}$. Assim $a=-\frac{k t_{1}}{t_{2}}$. Queremos provar que o $k$ é igual a 1 , mas vamos supor que isso não acontece, i.e., vamos supor que $k \geq 2$.

Seja $s \in \mathbb{R}$, então

$$
\begin{aligned}
\omega \bar{\omega}_{1} \omega^{-1} \alpha_{2}(s) & =\omega \bar{\omega}_{1} \alpha_{1}(a s+b) & & \text { dado que } \omega \alpha_{1}(a t+b)=\alpha_{2}(t) \\
& =\omega \alpha_{1}\left(a s+b+t_{1}\right) & & \text { pois }\left(\bar{\omega}_{1},\left.\alpha_{1}\right|_{\left[0, t_{1}\right]}\right) \text { es uma geodésica fechada } \\
& =\omega \alpha_{1}\left(a\left(s+\frac{t_{1}}{a}\right)+b\right) & & \\
& =\alpha_{2}\left(s+\frac{t_{1}}{a}\right) & & \text { dado que } \omega \alpha_{1}(a t+b)=\alpha_{2}(t) \\
& =\alpha_{2}\left(s-\frac{t_{2}}{k}\right) & & \text { pois } a=-\frac{k t_{1}}{t_{2}} .
\end{aligned}
$$

Portanto $\omega \bar{\omega}_{1}^{-1} \omega^{-1} \alpha_{2}\left(s-\frac{t_{2}}{k}\right)=\alpha_{2}(s)$, para todo $s \in \mathbb{R}$. Agora, dado que $-\frac{t_{2}}{k}<0$ e pela Proposição 3.4.1, obtemos que $\left(\omega \bar{\omega}_{1}^{-1} \omega^{-1},\left.\alpha_{2}\right|_{\left[0, \frac{t_{2}}{k}\right]}\right)$ é uma geodésica fechada em $X$. Assim, dado que $k \geq 2$, temos que $\frac{t_{2}}{k}<t_{2}$, e portanto $\left(\omega \bar{\omega}_{1}^{-1} \omega^{-1},\left.\alpha_{2}\right|_{\left[0, \frac{t_{2}}{k}\right]}\right)$ é uma geodésica primitiva. Mas isso é um absurdo, pois $\left(\bar{\omega}_{2},\left.\alpha_{2}\right|_{\left[0, t_{2}\right]}\right)$ é geodésica primitiva. Logo temos que $k=1$, e portanto $a=-\frac{t_{1}}{t_{2}}$.

Lema 3.4.2 Sejam $X=M / \Gamma$ um good orbifold Riemanniano e $\left(\omega_{1},\left.\alpha_{1}\right|_{[0,1]}\right)$ e $\left(\omega_{2},\left.\alpha_{2}\right|_{[0,1]}\right)$ duas geodésicas fechadas geometricamente iguais em X. Sejam $\left(\bar{\omega}_{1},\left.\alpha_{1}\right|_{\left[0, t_{1}\right]}\right)$ e $\left(\bar{\omega}_{2},\left.\alpha_{2}\right|_{\left[0, t_{2}\right]}\right)$ geodésicas fechadas primitivas de $\left(\omega_{1},\left.\alpha_{1}\right|_{[0,1]}\right)$ e $\left(\omega_{2},\left.\alpha_{2}\right|_{[0,1]}\right)$, respectivamente. Então

$$
\mathcal{L}\left(\left.\alpha_{i}\right|_{[0,1]}\right)=t_{i}^{-1} \mathcal{L}\left(\left.\alpha_{i}\right|_{\left[0, t_{i}\right]}\right) \text {, para } i=1,2 \text {, respectivamente. }
$$

Além disso

$$
\mathcal{L}\left(\left.\alpha_{1}\right|_{\left[0, t_{1}\right]}\right)=\mathcal{L}\left(\left.\alpha_{2}\right|_{\left[0, t_{2}\right]}\right)
$$

onde $\mathcal{L}(\cdot)$ denota o comprimento de uma curva em $M$.

Demonstração. Para cada $i \in\{1,2\}$ temos que $\mathcal{L}\left(\left.\alpha_{i}\right|_{[0,1]}\right)=n_{i} \mathcal{L}\left(\left.\alpha_{i}\right|_{\left[0, t_{i}\right]}\right)$, pois, pela Proposição 3.4.4, sabemos que $t_{i}=\frac{1}{n_{i}}$, onde $n_{i} \in\{1,2,3, \ldots\}$. Assim está provada a primeira parte. Vamos provar a segunda parte do lemma. Pela Proposição 3.4 .8 temos que $|a|=\frac{t_{1}}{t_{2}}$, onde

$$
\alpha_{2}(t)=\omega \alpha_{1}(a t+b) \text {, para todo } t \in \mathbb{R} \text {. }
$$


Então precisamos trabalhar nos dois seguintes casos: $a=\frac{t_{1}}{t_{2}}$ e $a=-\frac{t_{1}}{t_{2}}$.

1. Seja $a=\frac{t_{1}}{t_{2}}$. Então temos que

$$
\begin{aligned}
\mathcal{L}\left(\left.\alpha_{2}\right|_{\left[0, t_{2}\right]}\right) & =\int_{0}^{t_{2}}\left|\dot{\alpha}_{2}(t)\right| d t=\int_{b}^{t_{1}+b}\left|d \omega \dot{\alpha}_{1}(t)\right| d t \\
& =\int_{0}^{t_{1}}\left|\dot{\alpha}_{1}(t)\right| d t \\
& =\mathcal{L}\left(\left.\alpha_{1}\right|_{\left[0, t_{1}\right]}\right) .
\end{aligned}
$$

2. Se $a=-\frac{t_{1}}{t_{2}}$, temos que

$$
\begin{aligned}
\mathcal{L}\left(\left.\alpha_{2}\right|_{\left[0, t_{2}\right]}\right) & =\int_{0}^{t_{2}}\left|\dot{\alpha}_{2}(t)\right| d t=\int_{-t_{1}+b}^{b}\left|d \omega \dot{\alpha}_{1}(t)\right| d t \\
& =\int_{-t_{1}}^{o}\left|\dot{\alpha}_{1}(t)\right| d t=\int_{0}^{t_{1}}\left|\dot{\alpha}_{1}(t)\right| d t \\
& =\mathcal{L}\left(\left.\alpha_{1}\right|_{\left[0, t_{1}\right]}\right) .
\end{aligned}
$$

Observemos que nos dois casos obtimos que $\mathcal{L}\left(\left.\alpha_{1}\right|_{\left[0, t_{1}\right]}\right)=\mathcal{L}\left(\left.\alpha_{2}\right|_{\left[0, t_{2}\right]}\right)$. Logo temos a prova da segunda parte do lema.

Observação 3.4.9 Denotaremos por $\left[\left(\omega_{1},\left.\alpha_{1}\right|_{[0,1]}\right)\right]$, ou simplesmente por $\left[\alpha_{1}\right]$, ao conjunto de geodésicas fechadas em $X$ que são geometricamente iguais à geodésica fechada $\left(\omega_{1},\left.\alpha_{1}\right|_{[0,1]}\right)$. Logo vamos ter que, se $\left(\bar{\omega}_{1},\left.\alpha_{1}\right|_{\left[0, t_{1}\right]}\right)$ e $\left(\bar{\omega}_{2},\left.\alpha_{2}\right|_{\left[0, t_{2}\right]}\right)$ são geodésicas primitivas de $\left(\omega_{1},\left.\alpha_{1}\right|_{[0,1]}\right)$ e $\left(\omega_{2},\left.\alpha_{2}\right|_{[0,1]}\right)$, respectivamente, e $\left(\omega_{2},\left.\alpha_{2}\right|_{[0,1]}\right) \in\left[\alpha_{1}\right]$, então $\left(\bar{\omega}_{1},\left.\alpha_{1}\right|_{\left[0, t_{1}\right]}\right)$ e $\left(\bar{\omega}_{2},\left.\alpha_{2}\right|_{\left[0, t_{2}\right]}\right) s \tilde{a} o$ elementos de $\left[\alpha_{1}\right]$. Além disso, pelo Lema 3.4.2, temos que $\mathcal{L}\left(\left.\alpha_{1}\right|_{\left[0, t_{1}\right]}\right)=\mathcal{L}\left(\left.\alpha_{2}\right|_{\left[0, t_{2}\right]}\right)$. Então podemos dar a seguinte definição.

Definição 3.4.6 Sejam $X=M / \Gamma$ um good orbifold Riemanniano e $\left(\omega,\left.\alpha\right|_{[0,1]}\right)$ uma geodésica fechada em $X$. Vamos supor que $\left(\omega_{0},\left.\alpha\right|_{\left[0, t_{0}\right]}\right)$ é uma geodésica primitiva de $\left(\omega,\left.\alpha\right|_{[0,1]}\right)$, então definimos

$$
\mathcal{L}_{X}\left(\left.\alpha\right|_{[0,1]}\right):=\mathcal{L}_{X}([\alpha]):=\mathcal{L}\left(\left.\alpha\right|_{\left[0, t_{0}\right]}\right)
$$

Teorema 3.4.1 Seja $X=M / \Gamma$ um good orbifold Riemanniano. Vamos supor que existe um subgrupo $\Gamma_{0}$ de $\Gamma$ de indice finito. Então se $M / \Gamma_{0}$ admite infinitas geodésicas geometricamente distintas, temos que em $X$ existem infinitas geodésicas fechadas geometricamente distintas. 
Demonstração. Por hipótese existe uma sequência infinita $\left\{\left(\omega_{i},\left.\alpha_{i}\right|_{[0,1]}\right)\right\}_{i \in \mathbb{N}}$ de geodésicas fechadas geometricamente distintas dois a dois em $M / \Gamma_{0}$.

Afirmamos existem infinitas classes em $M / \Gamma$ tal que cada classe contém no máximo um número finito de elementos de $\left\{\left(\omega_{i},\left.\alpha_{i}\right|_{[0,1]}\right)\right\}_{i \in \mathbb{N}}$. E vamos provar a veracidade da afirmação pelo absurdo, i.e., vamos supor que existe apenas um número finito de classes em $M / \Gamma$ ou existe pelo menos uma classe em $M / \Gamma$ que contém infinitos elementos de $\left\{\left(\omega_{i},\left.\alpha_{i}\right|_{[0,1]}\right)\right\}_{i \in \mathbb{N}}$. Assim, temos que existe uma subsequência de $\left\{\left(\omega_{i},\left.\alpha_{i}\right|_{[0,1]}\right)\right\}_{i \in \mathbb{N}}$ onde todos seus termos são geodésicas fechadas geometricamente iguais em $X$. Logo, por simplicidade, podemos supor que tal subsequência é a mesma sequência $\left\{\left(\omega_{i},\left.\alpha_{i}\right|_{[0,1]}\right)\right\}_{i \in \mathbb{N}}$.

Agora, fixemos um destes elementos, por exemplo, fixemos $\left(\omega_{1},\left.\alpha_{1}\right|_{[0,1]}\right)$. Seja $j \in \mathbb{N}$, tal que $j>1$. Dado que $\left(\omega_{1},\left.\alpha_{1}\right|_{[0,1]}\right)$ e $\left(\omega_{j},\left.\alpha_{j}\right|_{[0,1]}\right)$ são geometricamente iguais em $X$, então temos, pela Proposição 3.4.7, que existem $\omega_{j_{1}} \in \Gamma \backslash \Gamma_{0}$ e números reais $a_{j_{1}}$ e $b_{j_{1}}$ tais que

$$
\alpha_{1}(t)=\omega_{j_{1}} \alpha_{j}\left(a_{j_{1}} t+b_{j_{1}}\right) \text {, para todo } t \in \mathbb{R}
$$

Seja $k>1$. Então, aplicando a equação (3.10) para $k$ e $j$, obtemos que

$$
\omega_{j_{1}} \alpha_{j}\left(a_{j_{1}} t+b_{j_{1}}\right)=\alpha_{1}(t)=\omega_{k_{1}} \alpha_{k}\left(a_{k_{1}} t+b_{k_{1}}\right) \text {, para todo } t \in \mathbb{R} \text {. }
$$

Agora, se $k \neq j$, então afirmamos que $\omega_{k_{1}} \neq \omega_{j_{1}}$. Com efeito, pois se acontece o contrário, pela equação (3.11), temos que

$$
\alpha_{k}\left(a_{k_{1}} t+b_{k_{1}}\right)=\omega_{k_{1}}^{-1} \alpha_{1}(t)=\omega_{j_{1}}^{-1} \alpha_{1}(t)=\alpha_{j}\left(a_{j_{1}} t+b_{j_{1}}\right) \text {, para todo } t \in \mathbb{R} .
$$

Mas isto é absurdo, pois $\left(\omega_{k},\left.\alpha_{k}\right|_{[0,1]}\right)$ e $\left(\omega_{j},\left.\alpha_{j}\right|_{[0,1]}\right)$ são geometricamente distintas em $M / \Gamma_{0}$. Logo temos que se $k \neq j$, então $\omega_{k_{1}} \neq \omega_{j_{1}}$, e portanto $\left\{\omega_{j_{1}}\right\}_{j>1}$ é uma sequência de elementos de $\Gamma$ distintos dois a dois.

Agora, a cardinalidade de $\Gamma \backslash \Gamma_{0}$ pode ser finita ou infinita, e as duas possibilidades dependem somente da cardinalidade de $\Gamma_{0}$, pois $\Gamma \backslash \Gamma_{0}$ é finito se e somente se $\Gamma_{0}$ é finito, dado que, por hipótese, $\Gamma_{0}$ é de índice finito em $\Gamma$. Logo vamos trabalhar em cada um destes casos.

1. Vamos supor que $\Gamma \backslash \Gamma_{0}$ é finito. Portanto a família $\left\{\omega_{j_{1}}\right\}_{j>1}$ é finita, pois ela está contida em $\Gamma \backslash \Gamma_{0}$, mas isso é um absurdo, pois provamos que $\left\{\omega_{j_{1}}\right\}_{j>1}$ é uma família de elementos distintos dois a dois. 
2. Suponhamos que $\Gamma \backslash \Gamma_{0}$ é infinito. Dado que $\Gamma_{0}$ é de índice finito em $\Gamma$, existe um subconjunto finito $\left\{\bar{\omega}_{1}, \bar{\omega}_{2}, \ldots, \bar{\omega}_{l}\right\}$ de $\Gamma \backslash \Gamma_{0}$, tal que para cada $\omega \in \Gamma \backslash \Gamma_{0}$, existem $\tilde{\omega} \in \Gamma_{0}$ e $\bar{\omega} \in\left\{\bar{\omega}_{1}, \bar{\omega}_{2}, \ldots, \bar{\omega}_{l}\right\}$ tais que $\omega=\bar{\omega} \tilde{\omega}$, i.e.,

$$
\Gamma=\Gamma_{0} \dot{\dot{\omega}} \bar{\omega}_{1} \Gamma_{0} \dot{ப} \ldots \dot{\dot{\omega}} \bar{\omega}_{l} \Gamma_{0} .
$$

Em particular, cada $\omega_{j_{1}}=\bar{\omega}_{j_{1}} \tilde{\omega}_{j_{1}}$, onde $\tilde{\omega}_{j_{1}} \in \Gamma_{0}$ e $\bar{\omega}_{j_{1}} \in\left\{\bar{\omega}_{1}, \bar{\omega}_{2}, \ldots, \bar{\omega}_{l}\right\}$.

Afirmamos que se $k \neq j$, então $\bar{\omega}_{k_{1}} \neq \bar{\omega}_{j_{j}}$. Com efeito, se isto não acontece, i.e., se temos que $\bar{\omega}_{k_{1}}=\bar{\omega}_{j_{1}}$, então, dado qualquer $t_{0} \in \mathbb{R}$, temos o seguinte

$$
\begin{aligned}
\bar{\omega}_{j_{1}} \tilde{\omega}_{j_{1}} \alpha_{j}\left(a_{j} t_{0}+b_{j}\right) & =\omega_{j_{1}} \alpha_{j}\left(a_{j_{1}} t_{0}+b_{j_{1}}\right) & & \text { pois } \omega_{j_{1}}=\bar{\omega}_{j_{1}} \tilde{\omega}_{j_{1}} \\
& =\alpha_{1}\left(t_{0}\right) & & \text { pela equação (3.11) } \\
& =\omega_{k_{1}} \alpha_{k}\left(a_{k_{1}} t_{0}+b_{k_{1}}\right) & & \text { pela equação (3.11) } \\
& =\bar{\omega}_{k_{1}} \tilde{\omega}_{k_{1}} \alpha_{k}\left(a_{k_{1}} t_{0}+b_{k_{1}}\right) & & \text { dado que } \omega_{k_{1}}=\bar{\omega}_{k_{1}} \tilde{\omega}_{k_{1}} .
\end{aligned}
$$

Então, dado que $t_{0}$ é qualquer número real, temos que

$$
\tilde{\omega}_{j_{1}} \alpha_{j}\left(a_{j_{1}} t+b_{j_{1}}\right)=\tilde{\omega}_{k_{1}} \alpha_{k}\left(a_{k_{1}} t+b_{k_{1}}\right)
$$

para todo $t \in \mathbb{R}$ e $\tilde{\omega}_{j_{1}}$ e $\tilde{\omega}_{k_{1}}$ são elementos de $\Gamma_{0}$. Mas isto é um absurdo, pois $\left(\omega_{j},\left.\alpha_{j}\right|_{[0,1]}\right)$ e $\left(\omega_{k},\left.\alpha_{k}\right|_{[0,1]}\right)$ são geometricamente distintas em $M / \Gamma_{0}$. Portanto $\bar{\omega}_{k_{1}} \neq \bar{\omega}_{j_{1}}$.

Então existe uma aplica injetora de $\left\{\omega_{j_{1}}\right\}_{j>1}$ em $\left\{\bar{\omega}_{1}, \bar{\omega}_{2}, \ldots, \bar{\omega}_{l}\right\}$, que é um absurdo por cardinalidade, pois sabemos que $\left\{\omega_{j 1}\right\}_{j>1}$ é uma família de elementos distintos dois a dois.

Dado que em cada um dos casos obtivemos uma contradição, então existem infinitas classes em $M / \Gamma$ tal que cada classe contém no máximo um número finito de elementos de $\left\{\left(\omega_{i},\left.\alpha_{i}\right|_{[0,1]}\right)\right\}_{i \in \mathbb{N}}$. Logo, em particular, existe uma subsequência $\left\{\left(\omega_{i_{k}},\left.\alpha_{i_{k}}\right|_{[0,1]}\right)\right\}_{k \in \mathbb{N}}$ de $\left\{\left(\omega_{i},\left.\alpha_{i}\right|_{[0,1]}\right)\right\}_{i \in \mathbb{N}}$ tal que todos os elementos de $\left\{\left(\omega_{i_{k}},\left.\alpha_{i_{k}}\right|_{[0,1]}\right)\right\}_{k \in \mathbb{N}}$ são geodésicas fechadas geometricamente distintas dois a dois em $X$.

Corolário 3.4.1 Sejam $(M, \mathrm{~g})$ uma variedade Riemanniana completa e conexa que admite infinitas geodésicas fechadas geometricamente distintas e $\Gamma \subset I s o(M, \mathrm{~g})$ finito. Então o orbifold Riemanniano $X=M / \Gamma$ admite infinitas geodésicas fechadas geometricamente distintas.

Demonstração. Temos a prova a partir do Teorema 3.4.1. 
Exemplo 3.4.5 (Bola de Rugby) Sejam $M=\mathbb{S}^{2}$ com a métrica canônica $\mathrm{g}_{0}$ e $\Gamma$ o subgrupo de Iso $\left(M, \mathrm{~g}_{0}\right)$ gerado por $\omega$, onde $\omega$ uma rotação de $\pi$ graus em torno do eixo $z$ no sentido antihorário. Sejam e o elemento identidad de $\Gamma$ e $\beta:[0,1] \rightarrow M$ uma geodésica periódica não trivial distinta do equador e dos meridianos, então temos que $\left(e,\left.\beta\right|_{[0,1]}\right)$ é uma geodésica fechada em $X=M / \Gamma$. Seja $\alpha:[0,1] \rightarrow M$ qualquer meridiano, então $\left(e,\left.\alpha\right|_{[0,1]}\right)$ é uma geodésica fechada em $X$ e é geometricamente distinta da geodésica fechada $\left(e,\left.\beta\right|_{[0,1]}\right)$. Agora seja $p \in M$ om ponto no equador, e observe que se $\gamma:[0,1] \rightarrow M$ é uma geodésica contida no equador, ligando $p$ e $-p$, i.e., $\gamma(0)=p \gamma(1)=-p$ de comprimento igual a $\pi$, então temos que $\left(\omega,\left.\gamma\right|_{[0,1]}\right)$ é uma geodésica fechada geométricamente distinta dàs anteriores. Além disso, observe que para qualquer geodésica $\gamma^{n}$ que é "múltiplo"da geodésica $\gamma$, i.e., $\gamma:[0, n] \rightarrow M$, temos que $\left(\omega,\left.\gamma\right|_{[0, n]}\right)$ é uma geodésica fechada em $X$, e são as únicas que são invariantes pela isometria $\omega$.

Neste exemplo, sem o uso do Corolário 3.4.1, é fácil ver que o good orbifold X possui infinitas geodésicas fechadas geometricamente distinas.

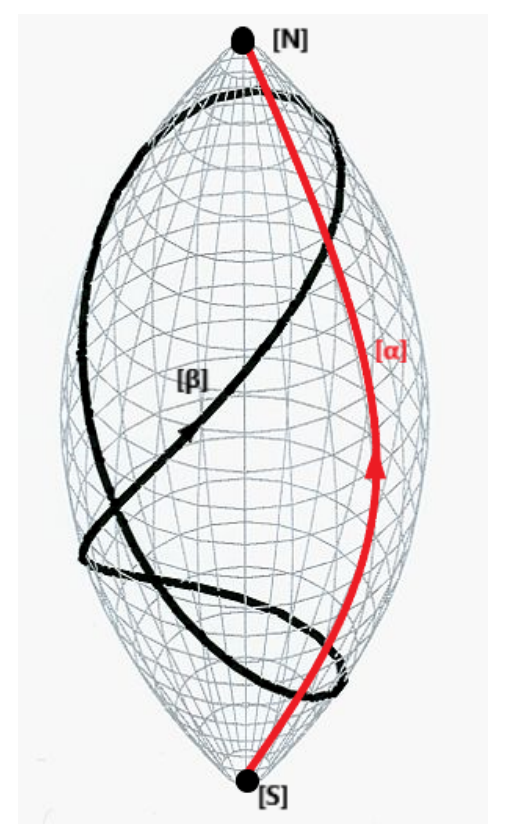

Figura 3.6: $O$ orbifold $X=M / \Gamma$ e as curvas $\pi(\beta)$ e $\pi(\alpha)$.

\subsection{Prova Detalhada do Teorema 2}

Lembremos a seguinte definição antes de dar nossa primeira proposição desta seção.

Definição 3.5.1 Sejam $(M, g)$ uma variedade Riemanniana e $\Gamma$ um subgrupo de Iso $(M, g)$. Dizemos que $\Gamma$ age cocompactamente sobre $M$ se existe um subconjunto compacto $K \subset M$ tal 
que

$$
M=\bigcup_{\omega \in \Gamma} \omega(K)
$$

Lema 3.5.1 Seja $X=M / \Gamma$ um good orbilfold Riemanniano. Então existe um domínio fundamental para a ação de $\Gamma$ sobre $M$.

Demonstração. Pela Proposição 1.2.3 temos que existe $x \in$ tal que $\Gamma_{x}=\{e\}$. Logo, pelo Teorema 1.3.2, existe um domínio de Dirichlet $D(x)$ para $\Gamma$ centrado em $x$ (vide Definição 1.3.3). Agora, pelo Teorema 1.3.2, temos que $D(x)$ é um domínio fundamental para $\Gamma$. Assim, definindo $D:=D(x)$, temos que $D$ é um domínio fundamental para a ação de $\Gamma$ sobre $M$.

Observação 3.5.1 Se $X=M / \Gamma$ é um good orbifold Riemanniano, então, pelo Lema 3.5.1, existe um dominio fundamental $D$ para $\Gamma$. Doravante sempre vamos supor a exitência de um dominio fundamental. Além disso, pelo Teorema 1.3.2, temos que D é localmente finito, i.e., $\{\omega(D): \omega \in \Gamma\}$ é uma família localmente finita.

Proposição 3.5.1 Seja $X=M / \Gamma$ um good orbifold Riemanniano. $X$ é compacto se, e somente se, $\Gamma$ age cocompactamente sobre $M$.

Demonstração. Primeiro vamos supor que $X$ é compacto. Sejam $\pi: M \rightarrow M / \Gamma$ a aplicação quociente e $D$ um domínio fundamental da ação de $\Gamma$ sobre $M$. Lembremos que, por definição, $D$ é localmente finito se a família $\{\omega(\bar{D}): \omega \in \Gamma\}$ é localmente finita. Agora, dado que $M$ é uma variedade Riemanniana completa, temos, pelo Teorema 1.3.2, que $D$ é localmente finito. Portanto, dado que $M / \Gamma$ é compacto e aplicando o Teorema 1.3.1, temos que $\bar{D}$ é compacto. Então, pela definição de $D$, temos que $M=\bigcup_{\omega \in \Gamma} \omega(\bar{D})$. Portanto $\Gamma$ age cocompactamente sobre $M$.

Vamos provar agora a recíproca da proposição. Supomos que existe um subconjunto compacto $K$ em $M$ tal que $M=\bigcup_{\omega \in \Gamma} \omega(K)$. Então temos que $\pi(K)=M / \Gamma$. Dado que $K$ é compacto e $\pi$ é contínua, temos que $\pi(K)$ é compacto, e portanto $M / \Gamma$ é um espaço compacto.

Proposição 3.5.2 Seja $X=M / \Gamma$ um good orbifold Riemanniano. Se $X$ é compacto e $D$ é um dominio fundamemtal para $\Gamma$, então $\bar{D}$ é compacto.

Demonstração. Segue-se da primeira parte da prova da Proposição 3.5.1. 
Proposição 3.5.3 Seja $X=M / \Gamma$ um good orbifold Riemanniano. Supomos que $\Gamma_{0}$ é um subgrupo de $\Gamma$ de indice finito, então $X$ é compacto se e somente se $M / \Gamma_{0}$ é compacto.

Demonstração. Definamos $\pi: M \rightarrow M / \Gamma$ e $\eta: M \rightarrow M / \Gamma_{0}$ as aplicações quocientes das ações sobre $M$ dos grupos $\Gamma$ e $\Gamma_{0}$, respectivamente.

Primeiro vamos supor que $M / \Gamma_{0}$ é compacto. Definamos a seguinte aplicação

$$
\begin{aligned}
\phi: & M / \Gamma_{0} \\
\Gamma_{0}(x) & \longrightarrow M / \Gamma \\
& \longrightarrow \Gamma(x) .
\end{aligned}
$$

Assim, $\phi$ é sobrejetora. Agora, pela definição de $\phi$, temos que $\pi=\phi \circ \eta$. Dado que $\pi$ é contínua e $\eta$ é uma aplicação quociente, temos que $\phi$ é contínua. Dado que $\phi$ é sobrejetora e contínua, e que $M / \Gamma_{0}$ é compacto, temos que $X$ é compacto.

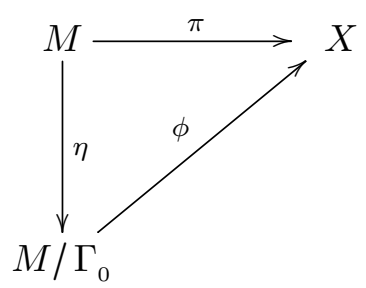

Agora vamos provar a recípoca da proposição. Supomos que $M / \Gamma$ é compacto, e seja $D$ un domínio fundamental para $\Gamma$. Dado que $M$ é uma variedade Riemanniana completa, temos que, pelo Teorema 1.3.2, $D$ é localmente finito, e portanto, dado que $M / \Gamma$ é compacto, e, pelo Teorema 1.3.1, temos que $\bar{D}$ é compacto. Agora, como $\Gamma_{0}$ é de índice finito em $\Gamma$, existem $\bar{\omega}_{1}, \bar{\omega}_{2}, \ldots, \bar{\omega}_{l}$ em $\Gamma \backslash \Gamma_{0}$, tais que $\Gamma=\Gamma_{0} \bigsqcup_{1 \leq n \leq l} \bar{\omega}_{n} \Gamma_{0}$. Definindo $K:=\bigcup_{1 \leq n \leq l} \bar{\omega}_{n}^{-1}(\bar{D})$, temos que $K$ é um subconjunto compacto de $M$. Seja $x$ qualquer ponto de $M$, mas fixo. Sabemos que existe $\omega \in \Gamma$ tal que $\omega(x) \in \bar{D}$. Agora, existem $\hat{\omega} \in \Gamma_{0}$ e $\bar{\omega} \in\left\{\bar{\omega}_{1}, \ldots, \bar{\omega}_{l}\right\}$ tais que $\omega=\bar{\omega} \hat{\omega}$, logo $\bar{\omega} \hat{\omega}(x) \in \bar{D}$. Portanto $\hat{\omega}(x) \in \bar{\omega}^{-1} \bar{D}$, e então $\hat{\omega}(x) \in K$. Dado que $x$ é qualquer elemento de $M$ e $\hat{\omega}$ é um elemento de $\Gamma_{0}$, temos que $M=\bigcup_{\omega \in \Gamma_{0}} \omega(K)$. Assim, pela Proposição 3.5.1, temos que $M / \Gamma_{0}$ é compacto. Logo vale a recíproca da proposição.

Teorema 3.5.1 Seja $X=M / \Gamma$ um good orbifold Riemanniano compacto, onde $M$ é uma variedade Riemanniana tal que $\operatorname{dim}(M)>1$ e $\Gamma$ um grupo infinito. Vamos supor que $\Gamma_{0}$ é um subgrupo abeliano $e$ de indice finito em $\Gamma$. Então $X$ admite uma família $\left\{\left(\omega_{i},\left.\alpha_{i}\right|_{[0,1]}\right)\right\}_{i \in \mathbb{N}}$ de geodésicas fechadas geometricamente distintas dois a dois. 
Demonstração. Dado que $X$ é compacto e que $\Gamma_{0}$ é de índice finito em $\Gamma$, obtemos, pela Proposição 3.5.3, que $M / \Gamma_{0}$ é compacto. Logo, pela proposição 3.5.1, existe um subconjunto compacto $C$ de $M$ tal que $M=\bigcup_{\omega \in \Gamma_{0}} \omega(C)$. Sejam $x \in C$ e $r>0$ tais que $C \subset B_{r}(x)$, então $M=\bigcup_{\omega \in \Gamma_{0}} \omega\left(B_{r}(x)\right)$. Assim, por [BH99] (Teorema I - 8.10) temos que $\Gamma_{0}$ é finitamente gerado. Também podemos obter que $\Gamma_{0}$ é finitamente gerado apartir do item número 5 da Proposição 2.2.1. Agora, por hipótese, $\Gamma_{0}$ é abeliano, e dado que $\Gamma_{0}$ é finitamente gerado, obtemos, por [Rot95] (Teorema 10.20), que

$$
\Gamma_{0} \approx T\left(\Gamma_{0}\right) \oplus \mathbb{Z}^{k}
$$

onde $T\left(\Gamma_{0}\right)$ é o subgrupo finito de torção de $\Gamma_{0}$ e $k$ é menor o igual ao número de geradores de $\Gamma_{0}$ e é chamado o posto de $\Gamma_{0}$.

Dado que $\Gamma$ é infinito e $\Gamma_{0}$ é de índice finito em $\Gamma$, vamos ter que $k \geq 1$. Além disso, pela Proposição 3.5.3, obtemos que $M / \mathbb{Z}^{k}$ é uma variedade Riemanniana compacta, pois $\mathbb{Z}^{k}$ é de índice finito em $\Gamma$. A prova do teorema vai ser obtida a partir do valor de $k$.

1. Vamos supor que $k=1$. Por [BH84] (vide resultado principal) temos que existem infinitas geodésicas fechadas geometricamente distintas na variedade $M / \mathbb{Z}$. Dado que $\mathbb{Z}$ é de índice finito em $\Gamma_{0}$ e $\Gamma_{0}$ é de índice finito em $\Gamma$, temos que $\mathbb{Z}$ é de índice finito em $\Gamma$. Assim, pelo Teorema 3.4.1, temos que o good orbifold Riemanniano $X$ admite infinitas geodésicas fechadas geometricamente distintas.

2. Supomos que $k \geq 2$. Sabemos que $\mathbb{Z}^{k}$ é um grupo abeliano livre de posto $k$. Seja então $\mathcal{A}=\left\{v_{1}, \ldots, v_{k}\right\}$ uma base para $\mathbb{Z}^{k}$.

Para cada $i \in \mathbb{N}$, definamos

$$
\omega_{i}=v_{1}+v_{2}+\cdots+v_{k-1}+i v_{k} .
$$

Assim, para cada $i \in \mathbb{N}$, temos que $\omega_{i}: M \rightarrow M$ é uma isometria de ordem infinita. Dado que $\Gamma$ age cocompactamente sobre $M$ (e, pela Proposição 3.5.1, acontece que $\Gamma_{0}$ também age cocompactamente sombre M), temos, pelo item número 3 da Proposição 2.2.1, que $\omega_{i}$ é um elemento hiperbólico, i.e., $\left|\omega_{i}\right|>0$ e Min $\left(\omega_{i}\right)$ é um conjunto não vazio. Seja, então, $x_{i} \in \operatorname{Min}\left(\omega_{i}\right)$. Dado que $M$ é uma variedade Riemanniana completa, seja $\alpha_{i}:[0,1] \rightarrow M$ uma geodésica minimizante ligando $x_{i}$ e $\omega_{i}\left(x_{i}\right)$, i.e., $\mathcal{L}\left(\left.\alpha_{i}\right|_{[0,1]}\right)=\left|\omega_{i}\right|$. Pelo item número 2 
do Teorema 2.4.1, temos que $\left(\omega_{i},\left.\alpha_{i}\right|_{[0,1]}\right)$ é uma geodésica fechada em $X$. Portanto

$$
\left\{\left(\omega_{i},\left.\alpha_{i}\right|_{[0,1]}\right)\right\}_{i \in \mathbb{N}}
$$

é uma família infinita de geodésicas fechadas em $M / \mathbb{Z}^{k}$. O seguinte lema (Lema 3.5.2), vai garantir que a sequência $\left\{\left(\omega_{i},\left.\alpha_{i}\right|_{[0,1]}\right)\right\}_{i \in \mathbb{N}}$ é uma familía de geodésicas fechadas geometricamente distintas dois a dois em $M / \mathbb{Z}^{k}$. E assim, aplicando o Teorema 3.4.1, obtemos que existe uma subsequência

$$
\left\{\left(\omega_{i_{k}},\left.\alpha_{i_{k}}\right|_{[0,1]}\right)\right\}_{k \in \mathbb{N}}
$$

de $\left\{\left(\omega_{i},\left.\alpha_{i}\right|_{[0,1]}\right)\right\}_{i \in \mathbb{N}}$ onde todos seus elementos são geodésicas fechadas geometricamente distintas dois a dois em $X$, e portanto temos a prova do Teorema.

Lema 3.5.2 Seja $M / \mathbb{Z}^{k}$, para $k \geq 2$, do Teorema 3.5.1. E seja a sequência $\left\{\omega_{i}\right\}_{i \in \mathbb{N}}$ como definimos na prova do teorema anterior. Então temos as seguintes propriedades de $\left\{\omega_{i}\right\}_{i \in \mathbb{N}}$ em $M / \mathbb{Z}^{k}$ :

1. Se $\omega_{i} \neq \omega_{j}$, então $\omega_{i}^{l} \neq \omega_{j}^{m}$, para todo par $l, m \in \mathbb{Z} \backslash\{0\}$.

2. Se $\left(\omega_{i},\left.\beta_{i}\right|_{[0,1]}\right)$ é uma geodésica fechada em $M / \mathbb{Z}^{k}$, e $\left(\bar{\omega}_{i},\left.\beta_{i}\right|_{\left[0, t_{i}\right]}\right)$ é uma geodésica fechada primitiva associada à geodésica fechada $\left(\omega_{i},\left.\beta_{i}\right|_{[0,1]}\right)$ em $M / \mathbb{Z}^{k}$, então $\bar{\omega}_{i}=\omega_{i}$ e $t_{i}=1$; i.e., $\left(\omega_{i},\left.\beta_{i}\right|_{[0,1]}\right)$ não admite geodésica primitiva distinta dela mesma.

3. Se $\omega_{i} \neq \omega_{j}$, e $\left(\omega_{i},\left.\beta_{i}\right|_{[0,1]}\right)$ e $\left(\omega_{j},\left.\beta_{j}\right|_{[0,1]}\right)$ são geodésicas fechadas em $M / \mathbb{Z}^{k}$, então elas são geometricamente distintas em $M / \mathbb{Z}^{k}$, i.e., a familia $\left\{\omega_{i}\right\}_{i \in \mathbb{N}}$ gera uma família infinita de geodesicas fechadas geometricamente distintas dois a dois em $M / \mathbb{Z}^{k}$.

Demonstração. Vamos supor que existem $l$ e $m \in \mathbb{Z}^{*}$ tais que $\omega_{i}^{l}=\omega_{j}^{m}$. Então

$$
l v_{1}+l v_{2}+\cdots+l v_{k-1}+l i v_{k}=m v_{1}+m v_{2}+\cdots+m v_{k-1}+m j v_{k}
$$

Dado que $\mathcal{A}$ é uma base para $\mathbb{Z}^{k}$, temos que (3.12) acontece se e somente se $l=m$ e $l i=m j$. Portanto $i=j$, mas isso é um absurdo pois $\omega_{i} \neq \omega_{j}$. Logo temos a prova do item número 1.

Vamos supor que $\left(\bar{\omega}_{i},\left.\beta_{i}\right|_{\left[0, t_{i}\right]}\right)$ é uma geodésica fechada primitiva associada à geodésica fechada $\left(\omega_{i},\left.\beta_{i}\right|_{[0,1]}\right)$. Sabemos, pela Proposição 3.4 .4 , que $t_{i}=\frac{1}{n_{i}}$, onde $n_{i} \in\{1,2,3, \ldots\}$. Temos, dado que 
$\left(\bar{\omega}_{i},\left.\beta_{i}\right|_{\left[0, t_{i}\right]}\right)$ é geodésica primitiva de $\left(\omega_{i},\left.\beta_{i}\right|_{[0,1]}\right)$ e pela Proposição 3.4.3, que

$$
\bar{\omega}_{i}^{n_{i}} \alpha_{i}(0)=\alpha_{i}(1)=\omega_{i} \alpha_{i}(0)
$$

Agora, dado que $\mathbb{Z}^{k}$ age livremente sobre $M$, obtemos que $\bar{\omega}_{i}^{n_{i}}=\omega_{i}$. Vamos supor que

$$
\bar{\omega}_{i}=l_{1} v_{1}+l_{2} v_{2}+\cdots+l_{k-1} v_{k-1}+l_{k} v_{k}, \operatorname{com} l_{j} \in \mathbb{Z}, \text { para } 1 \leq j \leq k .
$$

Então

$$
\bar{\omega}_{i}^{n_{i}}=n_{i}\left(l_{1} v_{1}+l_{2} v_{2}+\cdots+l_{k-1} v_{k-1}+l_{k} v_{k}\right)=\omega_{i}=v_{i}+v_{2}+\cdots+v_{k-1}+i v_{k}
$$

Assim $n_{i} l_{j}=1$, para todo $k-1 \geq j \geq 1$. E, dado que $n_{i} \geq 1$, obtemos que $l_{j}=n_{i}=1$, para todo $j=1,2, \ldots, k-1$ e $l_{k}=i$. Então temos a prova do item número 2 .

Sejam $\omega_{i} \neq \omega_{j}$ e $\left(\omega_{i},\left.\beta_{i}\right|_{[0,1]}\right)$ e $\left(\omega_{j},\left.\beta_{j}\right|_{[0,1]}\right)$ geodésica fechadas em $M / \mathbb{Z}^{k}$. Vamos supor que tales geodésicas fechadas são geometricamente iguais em $M / \mathbb{Z}^{k}$. Pelo item anterior, temos que $\left(\omega_{i},\left.\beta_{i}\right|_{[0,1]}\right)$ e $\left(\omega_{j},\left.\beta_{j}\right|_{[0,1]}\right)$ não possuem geodésicas primitivas diferentes delas mesmas em $M / \mathbb{Z}^{k}$, logo, usando este fato e a Proposição 3.4.7, temos que existem $\omega \in \mathbb{Z}^{k}$ e $b \in \mathbb{R}$, tais que $\beta_{i}(t)=\omega \beta_{j}( \pm t+b)$, para todo $t \in \mathbb{R}$. Então precisamos trabalhar em dois casos.

- Vamos supor que $\beta_{i}(t)=\omega \beta_{j}(t+b)$, para todo $t \in \mathbb{R}$. Dado que $\left(\omega_{i},\left.\beta_{i}\right|_{[0,1]}\right)$ é uma geodésica fechada, temos que $\left(\omega_{i},\left.\omega \beta_{j}\right|_{[b, b+1]}\right)$ é uma geodésica fechada, e portanto $\left(\omega_{i},\left.\omega \beta_{j}\right|_{[0,1]}\right)$ também é uma geodésica fechada. Para cada $t \in \mathbb{R}$, temos o seguinte

$$
\begin{aligned}
\omega_{i} \omega \beta_{j}(t) & =\omega \beta_{j}(t+1) & & \text { dado que }\left(\omega_{i},\left.\omega \beta_{j}\right|_{[0,1]}\right) \text { é uma geodésica fechada } \\
& =\omega \omega_{j} \beta_{j}(t) & & \text { pois }\left(\omega_{j},\left.\beta_{j}\right|_{[0,1]}\right) \text { é uma geodésica fechada. }
\end{aligned}
$$

Dado que $\mathbb{Z}^{k}$ age livremente sobre $M$, temos que $\omega_{i} \omega=\omega \omega_{j}$. E, dado que $\mathbb{Z}^{k}$ é abeliano, temos que $\omega_{i}=\omega_{j}$. Mas isto não pode acontecer, pois contradiz a hipótese.

- Vamos supor o outro caso: $\beta_{i}(t)=\omega \beta_{j}(-t+b)$, para todo $t \in \mathbb{R}$. Neste caso, dado que $\left(\omega_{i},\left.\beta_{i}\right|_{[0,1]}\right)$ é uma geodésica fechada, então, para todo $t \in \mathbb{R}$, obtemos que

$$
\omega_{i} \omega \beta_{j}(-t+b)=\omega_{i} \beta_{i}(0)=\beta_{i}(1)=\omega \beta_{j}((-t+b)-1) .
$$


Portanto, para todo $t \in \mathbb{R}$, temos que

$$
\omega_{i}^{-1} \omega \beta_{j}((-t+b)-1)=\omega \beta_{j}(-t+b)
$$

Assim, definindo $s:=-t+b$, obtemos

$$
\omega_{i}^{-1} \omega \beta_{j}(s-1)=\omega \beta_{j}(s), \text { para todo } s \in \mathbb{R}
$$

Logo, pela Proposição 3.4.1, temos que $\left(\omega_{i}^{-1},\left.\omega \beta_{j}\right|_{[0,1]}\right)$ é uma geodésica fechada em $M / \mathbb{Z}^{k}$. Agora, seja $t \in \mathbb{R}$, então

$$
\begin{aligned}
\omega_{i}^{-1} \omega \beta_{j}(t) & =\omega \beta_{j}(t+1) & & \text { dado que }\left(\omega_{i}^{-1},\left.\omega \beta_{j}\right|_{[0,1]}\right) \text { é uma geodésica fechada } \\
& =\omega \omega_{j} \beta_{j}(t) & & \text { pois }\left(\omega_{j},\left.\beta_{j}\right|_{[0,1]}\right) \text { é uma geodésica fechada. }
\end{aligned}
$$

Dado que $\mathbb{Z}^{k}$ é um grupo abeliano e que age livremente sobre $M$, obtemos que $\omega_{i}^{-1}=\omega_{j}$. Mas isso é um absurdo, pois $\omega_{i} \neq \omega_{j}$ e pelo item número 1 .

$\operatorname{Logo}\left(\omega_{i},\left.\beta_{i}\right|_{[0,1]}\right)$ e $\left(\omega_{j},\left.\beta_{j}\right|_{[0,1]}\right)$ são geodésicas fechadas geometricamente distintas em $M / \mathbb{Z}^{k}$. Então temos a prova do item 3, e assim a prova do lema.

Observação 3.5.2 Observe que o Teorema 3.5.1 também pode ser obtido a partir da mistura de [BH84] e do Teorema 3.4.1, pois Bangert e Hingston mencionam no artigo que toda variedade compacta com grupo fundamental abeliano possui infinitas geodésicas geometricamente distintas, mas eles somente provam no caso em que o grupo fundamental é $\mathbb{Z}$. Assim, nossa prova pode ser vista como uma prova alternativa, no entanto, nossa prova dará uma ideia para as provas do Teorema 3.6.1 e da Proposição 3.7.2.

\subsection{Prova Detalhada do Teorema 3}

Proposição 3.6.1 Seja $X=M / \Gamma$ um good orbifold compacto tal que $\Gamma$ é um grupo abeliano. Supomos que existe uma família $\left\{\left(\omega_{i},\left.\alpha_{i}\right|_{[0,1]}\right)\right\}_{i \in \mathbb{N}}$ de geodésicas primitivas geometricamente distintas em $X$ tal que $\omega_{i} \neq \omega_{j}$ para todo par $i, j$, com $i \neq j$. Então

$$
\left\{\mathcal{L}\left(\left.\alpha_{i}\right|_{[0,1]}\right)\right\}_{i \in \mathbb{N}}
$$

é uma sequência real não limitada. Mais ainda, temos que $\left\{\left|\omega_{i}\right|\right\}_{i \in \mathbb{N}}$ é uma sequência não limitada. 
Demonstração. Vamos provar a proposição pelo abusurdo, i.e., vamos supor que existe $s>0$ tal que

$$
\mathcal{L}\left(\left.\alpha_{i}\right|_{[0,1]}\right) \leq s, \text { para todo } i \in \mathbb{N}
$$

Seja $D$ o domínio fundamental para a ação de $\Gamma$ sobre $M$. Uma vez que $M / \Gamma$ é compacto, temos, pelo Corolário 3.5.2, que $\bar{D}$ é compacto. Assim, definamos $R:=$ diámetro $(\bar{D})$.

Por outro lado, dado que $D$ é um domínio fundamental, temos que

$$
M=\bigcup_{\omega \in \Gamma} \omega(\bar{D})
$$

Assim, para cada $i \in \mathbb{N}$, existe $\tilde{\omega}_{i} \in \Gamma$ tal que $\tilde{\omega}_{i} \alpha_{i}(0) \in \bar{D}$. Seja $x_{0} \in \bar{D}_{0}$. Então afirmamos que, para cada $i \in \mathbb{N}$, temos

$$
\tilde{\omega}_{i}\left(\left.\alpha_{i}\right|_{\left[0, t_{i}\right]}\right) \subset \overline{B_{R+s}\left(x_{0}\right)} \text {. }
$$

Primeiro, antes de provar a última afirmação, observemos que $\tilde{\omega}_{i}\left(\alpha_{i}(0)\right) \in \bar{D} \subset \overline{B_{R+s}\left(x_{0}\right)}$, para cada $i \in \mathbb{N}$. Para cada $i \in \mathbb{N}$ e cada $0<t \leq t_{i}$, temos que

$$
\begin{aligned}
d\left(x_{0}, \tilde{\omega}_{i} \alpha_{i}(t)\right) & \leq d\left(x_{0}, \tilde{\omega}_{i} \alpha_{i}(0)\right)+d\left(\tilde{\omega}_{i} \alpha_{i}(0), \tilde{\omega}_{i} \alpha_{i}(t)\right) \\
& =d\left(x_{0}, \tilde{\omega}_{i} \alpha_{i}(0)\right)+d\left(\alpha_{i}(0), \alpha_{i}(t)\right) \\
& \leq R+s .
\end{aligned}
$$

E assim temos a afirmação.

Por outro lado, como $M$ é uma variedade Riemanniana completa, temos que $\overline{B_{R+s}\left(x_{0}\right)}$ é compacta. Então, dado que $\Gamma$ age propriamente sobre $M$, obtemos que

$$
\mathcal{S}:=\left\{\omega \in \Gamma: \omega\left(\overline{B_{R+s}\left(x_{0}\right)}\right) \cap \overline{B_{R+s}\left(x_{0}\right)} \neq \varnothing\right\} \text { é um conjunto finito. }
$$

Agora, afirmamos que cada $\tilde{\omega}_{i}$ é um elemento de $\mathcal{S}$. Com efeito, para cada $\tilde{\omega}_{i}$, temos que

$$
\begin{aligned}
\omega_{i} \tilde{\omega}_{i}\left(\alpha_{i}(0)\right) & =\tilde{\omega}_{i} \omega_{i}\left(\alpha_{i}(0)\right) & & \text { dado que } \Gamma \text { é abeliano } \\
& =\tilde{\omega}_{i}\left(\alpha_{i}\left(t_{i}\right)\right) & & \text { pois }\left(\omega_{i},\left.\alpha_{i}\right|_{\left[0, t_{i}\right]}\right) \text { é uma geodésica fechada. }
\end{aligned}
$$

Portanto, dado que $\tilde{\omega}_{i} \alpha_{i}(0)$ e $\tilde{\omega}_{i} \alpha_{i}\left(t_{i}\right)$ são elementos de $\overline{B_{R+s}\left(x_{0}\right)}$, temos a afirmação.

Agora, dado que $\mathcal{S}$ é um conjunto finito, temos que a família $\left\{\omega_{i}\right\}_{i \in \mathbb{N}}$ é finita. Em particular, existem índices $j \neq l$, tais que $\omega_{j}=\omega_{l}$. Mas isso contradiz a hipótese que $\left\{\omega_{i}\right\}_{i \in \mathbb{N}}$ é uma família de elementos dois a dois distintos. Portanto temos a prova da proposição. 
Observação 3.6.1 Observemos que, en geral, a Proposição 3.6.1 é falsa se na família de geodésicas fechadas primitivas geometricamente distintas $\left\{\left(\omega_{i},\left.\alpha_{i}\right|_{[0,1]}\right)\right\}_{i \in \mathbb{N}}$ no good orbifold $X$, acontece que $\left\{\omega_{i}\right\}_{i \text { in } \mathbb{N}}$ é uma família finita. Por exemplo, considere $X$ toro como o quociente de $\mathbb{R}^{2} / \Gamma$, onde $\Gamma$ é gerado pelas seguintes isometrias

$$
\begin{aligned}
& \omega_{1}: \mathbb{R}^{2} \longrightarrow \mathbb{R}^{2} \quad \omega_{2}: \mathbb{R}^{2} \longrightarrow \mathbb{R}^{2} \\
& (x, y) \longmapsto(x+1, y) \quad \omega \longmapsto(x, y+1)
\end{aligned}
$$

Definamos a seguinte familia de geodésicas fechadas primitivas $\left\{\left(\omega_{i},\left.\alpha_{i}\right|_{[0,1]}\right)\right\}_{i \in \mathbb{N}}$, onde $\omega_{i}=\omega_{2}$, para todo $i \in \mathbb{N}$ e $\alpha_{i}:[0,1] \rightarrow \mathbb{R}^{2}$ é o segmento ligando $\left(x_{i}, 0\right)$ e $\left(x_{i}, 1\right)$, onde $\left\{x_{i}\right\}_{i \text { in } \mathbb{N}}$ é uma familia de pontos distintos do intervalo $[0,1]$. Observe que neste caso $\mathcal{L}\left(\left.\alpha_{i}\right|_{[0,1]}\right)=1$, para todo $i \in \mathbb{N}$.

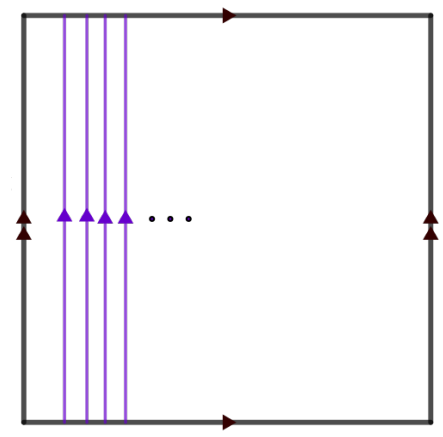

Figura 3.7: $O$ toro com algumas das infinitas geodésicas fechadas primitivas geometricamente distintas.

Teorema 3.6.1 Seja $X=M / \Gamma$ um good orbifold Riemanniano compacto, onde $M$ é uma variedade Riemanniana tal que $\operatorname{dim}(M)>1$ e $\Gamma$ é um grupo infinito. Vamos supor que existe um subgrupo $\Gamma_{0}$ de $\Gamma$ que é abeliano e de índice finito em $\Gamma$ e tal que o posto de $\Gamma_{0}$ é maior ou igual que 2. Então $X$ admite uma família $\left\{\left(\omega_{i},\left.\alpha_{i}\right|_{[0,1]}\right)\right\}_{i \in \mathbb{N}}$ de geodésicas fechadas geometricamente distintas dois a dois. E se definimos $X_{0}=M / \Gamma_{0}$, então $\left\{\mathcal{L}_{X_{0}}\left(\left.\alpha_{i}\right|_{[0,1]}\right)\right\}_{i \in \mathbb{N}}$ é uma sequência real não limitada. Em particular, a sequência $\left\{\left|\omega_{i}\right|\right\}_{i \in \mathbb{N}}$ é não limitada.

Demonstração. Primeiro observemos que, dado que $X$ é compacto e $\Gamma_{0}$ é de índice finito sobre $\Gamma$ e pela Proposição 3.5.3, $X_{0}$ é um good orbifold compacto. Observemos também que na demonstração do Teorema 3.5.1 obtemos que

$$
\Gamma_{0} \simeq T\left(\Gamma_{0}\right) \oplus \mathbb{Z}^{k}
$$

onde $k \geq 1$, pois $\Gamma$ é infinito e $\Gamma_{0}$ é de índice finito. Sendo $\mathcal{A}=\left\{v_{1}, \ldots, v_{k}\right\}$ uma base para $\mathbb{Z}^{k}$, com $k \geq 2$, e definindo $\omega_{i}=v_{1}+v_{2}+\cdots+v_{k-1}+i v_{k}$, para cada $i \in \mathbb{N}$, demonstramos (vide prova do Teorema 3.5.1, item número 2) que $\left\{\left(\omega_{i},\left.\alpha_{i}\right|_{[0,1]}\right)\right\}_{i \in \mathbb{N}}$ é uma sequência de geodésicas fechadas 
geometricamente distintas em $M / \mathbb{Z}^{k}$, onde $\mathcal{L}\left(\left.\alpha_{i}\right|_{[0,1]}\right)=\left|\omega_{i}\right|$.

Dado que $\mathbb{Z}^{k}$ é de índice finito em $\Gamma$, temos, pelo Teorema 3.4.1, que existe uma subsequência $\left\{\left(\omega_{i_{k}},\left.\alpha_{i_{k}}\right|_{[0,1]}\right)\right\}_{k \in \mathbb{N}}$ de $\left\{\left(\omega_{i},\left.\alpha_{i}\right|_{[0,1]}\right)\right\}_{i \in \mathbb{N}}$ tal que seus elementos são geodésicas fechadas geometricamente distintas dois a dois em $X$. E assim temos a prova da primeira parte do Teorema.

Agora vamos provar a segunda parte do teorema. Primeiro observemos que, pela Proposição ??, a sequência $\left\{\mathcal{L}\left(\left.\alpha_{i}\right|_{[0,1]}\right)\right\}_{i \in \mathbb{N}}$ é não limitada. Vamos supor que $\left\{\left(\bar{\omega}_{i},\left.\alpha_{i}\right|_{\left[0, t_{i}\right]}\right)\right\}_{i \in \mathbb{N}}$ é uma família de geodésicas primitivas associadas à família $\left\{\left(\omega_{i},\left.\alpha_{i}\right|_{[0,1]}\right)\right\}_{i \in \mathbb{N}}$ em $X_{0}$. Observe que o Lema 3.5.2 garante que, para cada $i \in \mathbb{N}$, a geodésica fechada $\left(\omega_{i},\left.\alpha_{i}\right|_{[0,1]}\right)$ não possui geodésica primitiva diferente dela mesma em $M / \mathbb{Z}^{k}$, mas não garante que acontece o mesmo em $X_{0}$. Portanto, para cada $i \in \mathbb{N}$, se $\bar{\omega}_{i} \neq \omega_{i}$, então $\bar{\omega}_{i} \in \Gamma_{0} \backslash \mathbb{Z}^{k}$. Agora, lembrando que (vide definição 3.7.1)

$$
\mathcal{L}_{X_{0}}\left(\left.\alpha_{i}\right|_{[0,1]}\right)=\mathcal{L}\left(\left.\alpha_{i}\right|_{\left[0, t_{i}\right]}\right),
$$

então nosso objetivo é provar que

$$
\lim _{i \rightarrow \infty} \mathcal{L}\left(\left.\alpha_{i}\right|_{\left[0, t_{i}\right]}\right) \rightarrow \infty
$$

Afirmamos que para cada par $j, l \in \mathbb{N}$, com $j \neq l$, temos que $\bar{\omega}_{j} \neq \bar{\omega}_{j}$. E vamos provar esta afirmação pelo absurdo, i.e., vamos supor que existe um par $j, l \in \mathbb{N}, \operatorname{com} j \neq l$, e tais que $\bar{\omega}_{j}=\bar{\omega}_{l}$. Já que $\left(\bar{\omega}_{j},\left.\alpha_{j}\right|_{\left[0, t_{j}\right]}\right)$ e $\left(\bar{\omega}_{l},\left.\alpha_{l}\right|_{\left[0, t_{l}\right]}\right)$ são geodésicas primitivas de $\left(\omega_{j},\left.\alpha_{j}\right|_{[0,1]}\right)$ e $\left(\omega_{l},\left.\alpha_{l}\right|_{[0,1]}\right)$, respectivamente, temos, pela Proposição 3.4.5, que existem números naturais $m_{j}, k_{j}, m_{l}, k_{l}$ tais que

$$
\bar{\omega}_{j}^{m_{j}}=\omega_{j}^{k_{j}} \text { e } \bar{\omega}_{l}^{m_{l}}=\omega_{l}^{k_{l}} .
$$

Assim, uma vez que $\bar{\omega}_{j}=\bar{\omega}_{l}$, obtemos que

$$
\omega_{j}^{m_{l} k_{l} k_{j} k_{j}}=\bar{\omega}_{j}^{m_{l} k_{l} m_{j} k_{j}}=\bar{\omega}_{l}^{m_{l} k_{l} m_{j} k_{j}}=\omega_{l}^{m_{j} k_{j} k_{l} k_{l}} .
$$

Portanto $\omega_{j}^{m_{l} k_{l} k_{j} k_{j}}=\omega_{l}^{m_{j} k_{j} k_{l} k_{l}}$. Mas isso é um absurdo pelo item número 1 do Lema 3.5.2. Logo todos o elementos da família $\left\{\bar{\omega}_{i}\right\}_{i \in \mathbb{N}}$ são distintos. Assim, pela Proposição 3.6.1, temos que a sequência $\left\{\mathcal{L}\left(\left.\alpha_{i}\right|_{\left[0, t_{i}\right]}\right)\right\}_{i \in \mathbb{N}}$ é não limitada. Agora, dado que

$$
\mathcal{L}\left(\left.\alpha_{i}\right|_{\left[0, t_{i}\right]}\right) \leq\left|\omega_{i}\right| \text {, para cada } i \in \mathbb{N},
$$


temos que a sequência $\left\{\left|\omega_{i}\right|\right\}_{i \in \mathbb{N}}$ é não limitada. E assim temos a prova do teorema.

A seguir daremos um exemplo onde podemos aplicar o Teorema 3.6.1 e o Corolário 1 (vide [Rat06], Teorema 7.5.2.).

Exemplo 3.6.1 Sejam $\mathbb{R}^{n}$ e $\Gamma$ um subgrupo discreto de isometrias de Iso $\left(\mathbb{R}^{n}\right)$ tais que $n>2$, $\Gamma$ age propriamente sobre $\mathbb{R}^{n}$ e $\mathbb{R}^{n} / \Gamma$ é compacto. Então o subgrupo de transalações $\mathbb{T}$ de $\Gamma$ é de índice finito e de posto $n$, portanto, pelo Teorema 3.6.1. temos que $\mathbb{R}^{n} / \Gamma$ possui uma familia $\left\{\left(\omega_{i},\left.\alpha_{i}\right|_{[0,1]}\right)\right\}_{i \in \mathbb{N}}$ de geodésicas fechadas geometricamente distintas dois a dois. Agora, pelo Corolário 1 , obtemos que $\left\{\mathcal{L}_{\mathbb{R}^{n} / \Gamma}\left(\left.\alpha_{i}\right|_{[0,1]}\right)\right\}_{i \in \mathbb{N}}$ é uma sequência real não limitada.

\subsection{Provas das Proposições 2 e 3}

Antes de enunciar os seguintes resultados, vamos lembrar uma definição importante para grupos finitamente gerados.

Definição 3.7.1 Seja $\Gamma$ um grupo finitamente gerado, e vamos supor que

$$
\mathcal{B}=\left\{\omega_{1}, \omega_{2}, \ldots, \omega_{n}: \omega_{i} \neq \text { e para todo } 1 \leq i \leq n\right\}
$$

é um conjunto que gera $\Gamma$. Definimos

$$
\begin{aligned}
& \mathrm{L}_{\mathcal{B}}: \quad \Gamma \quad \longrightarrow \mathbb{N} \cup\{0\} \\
& \omega \longmapsto \mathrm{L}_{\mathcal{B}}(\omega)
\end{aligned}
$$

onde $\mathrm{L}_{\mathcal{B}}(\omega)$ denota o menor comprimento entre as palavras em que pode ser escrita $\omega$ no dicionário dado pelo conjunto $\mathcal{B} \cup \mathcal{B}^{-1}$. E definimos $\mathrm{L}_{\mathcal{B}}(e):=0$, onde e é o elemento identidade de $\Gamma$.

Observação 3.7.1 É fácil ver que

$$
\mathrm{L}_{\mathcal{B}}\left(\omega_{1} \omega_{2}\right) \leq \mathrm{L}_{\mathcal{B}}\left(\omega_{1}\right)+\mathrm{L}_{\mathcal{B}}\left(\omega_{2}\right) \text {, para todo par } \omega_{1}, \omega_{2} \text { em } \Gamma \text {. }
$$

Assim, definimos a seguinte métrica sobre $\Gamma$

$$
\begin{aligned}
& d_{\mathcal{B}}: \quad \Gamma \times \Gamma \quad \longrightarrow \quad \mathbb{N} \cup\{0\} \\
& \left(\omega_{1}, \omega_{2}\right) \longmapsto \mathrm{L}_{\mathcal{B}}\left(\omega_{1}^{-1} \omega_{2}\right) .
\end{aligned}
$$


No Teorema 3.6.1 provamos que dada uma base $\mathcal{A}=\left\{v_{i}, v_{2}, \ldots, v_{k-1}, v_{k}\right\}$ de $\mathbb{Z}^{k}$, com $k \geq 2$, e definindo $\omega_{i}:=v_{1}+v_{2}+\cdots+v_{k-1}+i v_{k}$, então a sequência real $\left\{\left|\omega_{i}\right|\right\}_{i \in \mathbb{N}}$ é não limitada. Agora observemos que para cada $\omega_{i}$, temos que $\mathrm{L}_{A}\left(\omega_{i}\right)=i+k-1$. Portanto, temos que a sequência $\left\{\mathrm{L}_{A}\left(\omega_{i}\right)\right\}_{i \in \mathbb{N}}$ é também não limitada. Assim, a pergunta natural que segue é se existe alguma relação entre $\mathrm{L}_{A}\left(\omega_{i}\right)$ e $\left|\omega_{i}\right|$, e que tipo de relação é. Respondemos a esta pergunta na Proposição 3.7.1.

Antes de enunciar e provar a Proposição 3.7.1, vamos dar e provar o seguinte lema.

Lema 3.7.1 Se $X=M / \Gamma$ um good orbifold compacto, então $\Gamma$ é finitamente gerado. Além disso, se $\mathcal{A}=\left\{\omega_{1} \omega_{2}, \ldots \omega_{n}\right\}$ e $\mathcal{B}=\left\{\bar{\omega}_{1}, \bar{\omega}_{2}, \ldots, \bar{\omega}_{m}\right\}$ são dois conjuntos de geradores de $\Gamma$, então existe $\lambda \geq 1$ tal que

$$
\lambda^{-1} \mathrm{~L}_{\mathcal{B}}(\omega) \leq \mathrm{L}_{\mathcal{A}}(\omega) \leq \lambda \mathrm{L}_{\mathcal{B}}(\omega)
$$

para todo $\omega \in \Gamma$.

Demonstração. Já provamos no Teorema 3.5.1 que se $X=M / \Gamma$ é um good orbifold compacto, então o grupo $\Gamma$ é finitamente gerado.

Agora vamos provar a segunda parte do lema. Definamos $\lambda_{1}:=\max \left\{\mathrm{L}_{\mathcal{A}}\left(\bar{\omega}_{i}\right): 1 \leq i \leq m\right\}$ e $\lambda_{2}:=m x\left\{\mathrm{~L}_{\mathcal{B}}\left(\omega_{j}\right): 1 \leq j \leq n\right\}$. Seja $\omega$ qualquer elemento de $\Gamma$. Entao vamos supor que $\mathrm{L}_{\mathcal{A}}(\omega)=k$ e $\mathrm{L}_{\mathcal{B}}(\omega)=l$, e $\omega=\omega_{i_{1}}^{\epsilon_{1}} \omega_{i_{2}}^{\epsilon_{2}} \cdots \omega_{i_{k}}^{\epsilon_{k}}=\bar{\omega}_{j_{1}}^{\delta_{1}} \bar{\omega}_{j_{2}}^{\delta_{2}} \cdots \bar{\omega}_{j_{l}}^{\delta_{l}}$, onde $\omega_{i_{1}}, \omega_{i_{2}}, \ldots, \omega_{i_{k}} \in \mathcal{A}$, $\bar{\omega}_{j_{1}}, \bar{\omega}_{j_{1}}, \ldots, \bar{\omega}_{j_{l}} \in \mathcal{B}$ e $\epsilon_{1}, \ldots, \epsilon_{k}, \delta_{1}, \ldots, \delta_{l} \in\{ \pm 1\}$, i.e.

$$
\mathrm{L}_{\mathcal{A}}(\omega)=\mathrm{L}_{\mathcal{A}}\left(\omega_{i_{1}}^{\epsilon_{1}} \omega_{i_{2}}^{\epsilon_{2}} \cdots \omega_{i_{k}}^{\epsilon_{k}}\right)=k \quad \text { e } \quad \mathrm{L}_{\mathcal{B}}(\omega)=\mathrm{L}_{\mathcal{B}}\left(\bar{\omega}_{j_{1}}^{\delta_{1}} \bar{\omega}_{j_{2}}^{\delta_{2}} \cdots \bar{\omega}_{j_{l}}^{\delta_{l}}\right)=l
$$

Assim

$$
\mathrm{L}_{\mathcal{A}}(\omega)=\mathrm{L}_{\mathcal{A}}\left(\bar{\omega}_{j_{1}}^{\delta_{1}} \bar{\omega}_{j_{2}}^{\delta_{2}} \cdots \bar{\omega}_{j_{l}}^{\delta_{l}}\right) \leq \sum_{n=1}^{l} \mathrm{~L}_{\mathcal{A}}\left(\bar{\omega}_{j_{n}}^{\delta_{n}}\right) \leq \sum_{n=1}^{l} \lambda_{1}=\lambda_{1} l=\lambda_{1} \mathrm{~L}_{\mathcal{B}}(\omega)
$$

$\mathrm{e}$

$$
\mathrm{L}_{\mathcal{B}}(\omega)=\mathrm{L}_{\mathcal{B}}\left(\omega_{i_{1}}^{\epsilon_{1}} \omega_{i_{2}}^{\epsilon_{2}} \cdots \omega_{i_{k}}^{\epsilon_{k}}\right) \leq \sum_{n=1}^{k} \mathrm{~L}_{\mathcal{B}}\left(\omega_{i_{n}}^{\epsilon_{n}}\right) \leq \sum_{n=1}^{k} \lambda_{2}=\lambda_{2} k=\lambda_{2} \mathrm{~L}_{\mathcal{A}}(\omega)
$$

Definamos $\lambda:=\max \left\{\lambda_{1}, \lambda_{2}\right\}$. Então obtemos, a partir das equações (3.14) e (3.15), que

$$
\lambda^{-1} \mathrm{~L}_{\mathcal{B}}(\omega) \leq \lambda_{2}^{-1} \mathrm{~L}_{\mathcal{B}}(\omega) \leq \mathrm{L}_{\mathcal{A}}(\omega) \leq \lambda_{1} \mathrm{~L}_{\mathcal{B}}(\omega) \leq \lambda \mathrm{L}_{\mathcal{B}}(\omega)
$$

E assim, dado que $\omega$ é qualquer elemento de $\Gamma$, temos a prova do lema. 
Proposição 3.7.1 Seja $X=M / \Gamma$ uma variedade Riemanniana compacta, onde $\Gamma=\mathbb{Z}^{k}$, com $k \geq 2$. Vamos supor que

$$
\mathcal{A}=\left\{v_{1}, v_{2}, \ldots, v_{k}\right\}
$$

é uma base para $\Gamma$. Então existem constantes positivas $r$ e $P$, tais que

$$
P \mathrm{~L}_{\mathcal{A}}(\omega) \leq \frac{1}{r}|\omega|+1, \text { para todo } \omega \in \Gamma
$$

Além disso, se para cada $i \geq 1$, definimos

$$
\omega_{i}:=v_{1}+v_{2}+\cdots+v_{k-1}+i v_{k}
$$

então a sequência real $\left\{\left|\omega_{i}\right|\right\}_{i \geq 1}$ é não limitada.

Demonstração. Primeiro observemos que, dado que $M / \mathbb{Z}^{k}$ é compacta e pela Proposição 3.5.1, o grupo $\mathbb{Z}^{k}$ age cocompactamente sobre $M$. Assim, pelo item número 5 da Proposição 2.2.1, temos que $\mathbb{Z}^{k}$ é finitamente gerado. Assim, não está errado supor que existe uma base $\mathcal{A}=\left\{v_{1}, v_{2}, \ldots, v_{k}\right\}$ $\operatorname{para} \mathbb{Z}^{k}$.

Agora, pela Observação 3.5.1, temos que existe um domínio fundamental $D$ para a ação de $\Gamma$ em M. Então, pela definição 1.3.1, temos que

$$
M=\bigcup_{\omega \in \Gamma} \omega(\bar{D})
$$

Dado que $X$ é compacto, temos, pela Proposição 3.5.2, que $\bar{D}$ é compacto. Definamos então

$$
R:=\sup \{d(x, y): x, y \in \bar{D}\}
$$

i.e., $R$ é o diámetro de $\bar{D}$, e portanto $R$ é finito.

Seja $x_{0}$ qualquer elemento em $M$, mas fixo. Então existe $\omega_{0} \in \Gamma$ tal que $x_{0} \in \omega_{0}(\bar{D})$. Assim, temos que $\omega_{0}(\bar{D}) \subset \overline{B_{R}\left(x_{0}\right)}$, e portanto

$$
M=\bigcup_{\omega \in \Gamma} \omega\left(\overline{B_{R}\left(x_{0}\right)}\right) .
$$

Definamos

$$
\mathcal{B}_{0}:=\left\{\omega \in \Gamma: \omega \neq e \text { e } \omega\left(\overline{B_{R}\left(x_{0}\right)}\right) \cap \overline{B_{R}\left(x_{0}\right)} \neq \varnothing\right\}
$$


Então, dado que $M$ é uma variedade Riemanniana completa, temos que $\overline{B_{R}\left(x_{0}\right)}$ é compacta em $M$, e, dado que $\Gamma$ age propriamente e é discretro, então $\mathcal{B}_{0}$ é finito. Também observemos que $\mathcal{B}_{0}=\mathcal{B}_{0}^{-1}$. Afirmamos que $\mathcal{B}_{0}$ é não vazio. Com efeito, pois se $\mathcal{B}_{0}$ é vazio, então para qualquer par $\omega_{1}, \omega_{2}$ em $\Gamma$, com $\omega_{1} \neq \omega_{2}$, obtemos que

$$
\omega_{1}\left(\mathcal{B}_{0}\right) \cap \omega_{2}\left(\mathcal{B}_{0}\right)=\varnothing
$$

e portanto

$$
M=\bigcup_{\omega \in \Gamma} \omega\left(\overline{B_{R}\left(x_{0}\right)}\right)
$$

Mas isso é um absurdo, pois $M$ é uma variedade completa. Portanto $\mathcal{B}_{0}$ é não vazio.

Agora, seja $r_{0}$ definido da seguinte maneira

$$
r_{0}:=\inf \left\{d\left(\overline{B_{R}\left(x_{0}\right)}, \omega\left(\overline{B_{R}\left(x_{0}\right)}\right)\right): \omega \in \Gamma \backslash\left[\mathcal{B}_{0} \cup\{e\}\right]\right\} .
$$

Afirmação 1. $r_{0}>0$ e $r_{0}:=\min \left\{d\left(\overline{B_{R}\left(x_{0}\right)}, \omega\left(\overline{B_{R}\left(x_{0}\right)}\right)\right): \omega \in \Gamma \backslash\left[\mathcal{B}_{0} \cup\{e\}\right]\right\}$.

Sejam $\tilde{\omega} \in \Gamma \backslash\left[\mathcal{B}_{0} \cup\{e\}\right]$ e $s=d\left(\overline{B_{R}\left(x_{0}\right)}, \tilde{\omega}\left(\overline{B_{R}\left(x_{0}\right)}\right)\right)$. Dado que $\tilde{\omega} \in \Gamma \backslash\left[\mathcal{B}_{0} \cup\{e\}\right]$ e que $\overline{B_{R}\left(x_{0}\right)}$ é compacta, temos que $s>0$. Definamos

$$
\mathcal{T}:=\left\{\omega \in \Gamma \backslash\left[\mathcal{B}_{0} \cup\{e\}\right]: d\left(\overline{B_{R}\left(x_{0}\right)}, \omega\left(\overline{B_{R}\left(x_{0}\right)}\right)\right) \leq s\right\}
$$

Dado que $\tilde{\omega} \in \Gamma \backslash\left[\mathcal{B}_{0} \cup\{e\}\right]$, temos que $\mathcal{T}$ é não vazio. Agora, dado $\omega \in \mathcal{T}$, temos que $\omega\left(\overline{B_{R+s}\left(x_{0}\right)}\right) \cap$ $\overline{B_{R+s}\left(x_{0}\right)} \neq \varnothing$. Logo, dado que $\Gamma$ age propriamente e que $\overline{B_{R+s}\left(x_{0}\right)}$ é compacta, pois $M$ é completa, temos que $\mathcal{T}$ é finito. Também é fácil ver que, pela compacidade de $\overline{B_{R}\left(x_{0}\right)}$, cada $\omega \in \mathcal{T}$ satisfaz que $d\left(\overline{B_{R}\left(x_{0}\right)}, \omega\left(\overline{B_{R}\left(x_{0}\right)}\right)\right)>0$. Agora, pela definição de $r_{0}$ temos que

$$
r_{0}=\inf \left\{d\left(\overline{B_{R}\left(x_{0}\right)}, \omega\left(\overline{B_{R}\left(x_{0}\right)}\right)\right): \omega \in \mathcal{T}\right\}
$$

Dado que $\mathcal{T}$ é finito e que $d\left(\overline{B_{R}\left(x_{0}\right)}, \omega\left(\overline{B_{R}\left(x_{0}\right)}\right)\right)>0$ para cada $\omega \in \mathcal{T}$, temos que $r_{0}>0$ e $r_{0}=\min \left\{d\left(\overline{B_{R}\left(x_{0}\right)}, \omega\left(\overline{B_{R}\left(x_{0}\right)}\right)\right): \omega \in \mathcal{T}\right\}=\min \left\{d\left(\overline{B_{R}\left(x_{0}\right)}, \omega\left(\overline{B_{R}\left(x_{0}\right)}\right)\right): \omega \in \Gamma \backslash\left[\mathcal{B}_{0} \cup\{e\}\right]\right\}$.

Assim temos a prova da afirmação.

Afirmação 2. $r_{0} \leq 2 R$.

Vamos provar a afirmação pelo absurdo. Vamos supor, então, que $2 R<r_{0}$. Pela afirmação anterior 
temos que existe $\bar{\omega} \in \Gamma \backslash\left[\mathcal{B}_{0} \cup\{e\}\right]$ tal que $r_{0}=d\left(\overline{B_{R}\left(x_{0}\right)}, \bar{\omega}\left(\overline{B_{R}\left(x_{0}\right)}\right)\right)$. Observemos que se $\omega \in \mathcal{B}_{0} \cup\{e\}$, então $\overline{B_{R}\left(\omega\left(x_{0}\right)\right)} \subset \overline{B_{2 R}\left(\left(x_{0}\right)\right)}$. Agora, dado que estamos supondo que $2 R<r_{0}$, então temos que $\overline{B_{2 R}\left(\left(x_{0}\right)\right)} \cap \bar{\omega}\left(\overline{B_{R}\left(x_{0}\right)}\right)=\varnothing$, e assim $\overline{B_{2 R}\left(\left(x_{0}\right)\right)} \cap \omega\left(B_{R}\left(x_{0}\right)\right)=\varnothing$, para todo $\omega \in \Gamma \backslash\left[\mathcal{B}_{0} \cup\{e\}\right]$. Agora, dado que $M=\bigcup_{\omega \in \Gamma} \omega\left(\overline{B_{R}\left(x_{0}\right)}\right)$, então

$$
M=\overline{B_{2 R}\left(\left(x_{0}\right)\right)} \bigcup_{\omega \in \Gamma \backslash\left[\mathcal{B}_{0} \cup\{e\}\right]} \omega\left(\overline{B_{R}\left(x_{0}\right)}\right) .
$$

Onde o ponto na união significa que para todo $\omega \in \Gamma \backslash\left[\mathcal{B}_{0} \cup\{e\}\right]$, temos

$$
B_{2 R}\left(\left(x_{0}\right)\right) \cap \omega\left(B_{R}\left(x_{0}\right)\right)=\varnothing
$$

Mas isso é um absurdo, pois $M$ é uma variedade completa. Logo temos a afirmação.

Afirmação 3. $\mathcal{B}_{0}$ gera $\Gamma$ e

$$
\mathrm{L}_{\mathcal{B}_{0}}(\omega)=d_{\mathcal{B}_{0}}(e, \omega) \leq \frac{1}{R} d\left(x_{0}, \omega\left(x_{0}\right)\right)+1, \text { para todo } \omega \in \Gamma \text {. }
$$

Supomos primeiro que $\omega$ é um elemento de $\mathcal{B}_{0} \cup\{e\}$. Assim, claramamente, $\omega$ é gerado por elementos de $\mathcal{B}_{0} \cup \mathcal{B}_{0}^{-1}$. Além disso, $\omega$ atende a equação (3.22). Então resta provar a afirmação para os elementos de $\Gamma \backslash\left[\mathcal{B}_{0} \cup\{e\}\right]$.

Seja $\omega \in \Gamma \backslash\left[\mathcal{B}_{0} \cup\{e\}\right]$. Então temos que $R<d\left(\overline{B_{r}\left(x_{0}\right)}, \omega\left(\overline{B_{r}\left(x_{0}\right)}\right)\right)$. Portanto

$$
2 R+r_{0} \leq d\left(\omega\left(x_{0}\right), x_{0}\right)
$$

E assim

$$
R<d\left(\omega\left(x_{0}\right), x_{0}\right)
$$

Por propriedade Arquimediana dos números, seja $j \in \mathbb{N}$, tal que

$$
R(j-1) \leq d\left(\omega\left(x_{0}\right), x_{0}\right)<R j
$$

Observe que $j \geq 3$, pois $R<r_{0}+2 R \leq d\left(\overline{B_{r}\left(x_{0}\right)}, \omega\left(\overline{B_{r}\left(x_{0}\right)}\right)\right)$. Agora, dado que $M$ é uma variedade Riemanniana completa, existem $x_{1}, x_{2}, \ldots x_{j}, x_{j+1}$, onde $x_{j+1}:=\omega\left(x_{0}\right)$ e tais que

$$
d\left(x_{0}, x_{1}\right)<R \text { e } d\left(x_{l}, x_{l+1}\right)<r_{0} \text {, para todo } l=1,2, \ldots j .
$$


Agora, dado que $M=\bigcup_{\omega \in \Gamma} \omega\left(\overline{B_{R}\left(x_{0}\right)}\right)$, existem $e=\omega_{0}, \omega_{1}, \omega_{2}, \ldots \omega_{j-1}, \omega_{j}=\omega$ em $\Gamma$, tais que

$$
x_{l} \in \omega_{l-1}\left(\overline{B_{R}\left(x_{0}\right)}\right) \text { para todo } l=1,2, \ldots j .
$$

Definamos $\tilde{\omega}_{l}:=\omega_{l-1}^{-1} \omega_{l}$, para $l=1,2, \ldots j$. Agora, cada $i \in\{1,2, \ldots j\}$, atende que

$$
\begin{aligned}
d\left(\overline{B_{R}\left(x_{0}\right)}, \tilde{\omega}_{i}\left(\overline{B_{r}\left(x_{0}\right)}\right)\right) & \leq d\left(\omega_{i-1}^{-1}\left(x_{i}\right), \tilde{\omega}_{i} \omega_{i}^{-1}\left(x_{i+1}\right)\right) & & \text { pois } x_{l} \in \omega_{l-1}\left(\overline{B_{R}\left(x_{0}\right)}\right), \text { para } 1 \leq l \leq j \\
& =d\left(\omega_{i-1}^{-1}\left(x_{i}\right), \omega_{i-1}^{-1} \omega_{i} \omega_{i}^{-1}\left(x_{i+1}\right)\right) & & \text { pois } \tilde{\omega}_{l}:=\omega_{l-1}^{-1} \omega_{l}, \text { para } 1 \leq l \leq j \\
& =d\left(x_{i}, x_{i+1}\right)<r_{0} . & &
\end{aligned}
$$

Assim, dado que $r_{0}:=\min \left\{d\left(\overline{B_{R}\left(x_{0}\right)}, \omega\left(\overline{B_{R}\left(x_{0}\right)}\right)\right): \omega \in \Gamma \backslash\left[\mathcal{B}_{0} \cup\{e\}\right]\right\}$, temos que

$$
d\left(\overline{B_{r}\left(x_{0}\right)}, \tilde{\omega}_{i}\left(\overline{B_{r}\left(x_{0}\right)}\right)\right)=0, \text { para cada } i=1,2, \ldots j
$$

Logo $\tilde{\omega}_{i} \in \mathcal{B}_{0}$, para todo $i=1,2, \ldots j$.

Por outro lado, dado que

$$
\omega=\left(\omega_{0}^{-1} \omega_{1}\right)\left(\omega_{1}^{-1} \omega_{2}\right) \cdots\left(\omega_{j-2}^{-1} \omega_{j-1}\right)\left(\omega_{j-1}^{-1} \omega_{j}\right)=\tilde{\omega}_{1} \tilde{\omega}_{2} \cdots \tilde{\omega}_{j-1} \tilde{\omega}_{j}
$$

temos que $\omega$ é gerado por elementos de $\mathcal{B}_{0}$. Assim, como $\omega$ é qualquer elemento em $\Gamma \backslash\left[\mathcal{B}_{0} \cup\{e\}\right]$ e já tendo provado que cada elemento de $\mathcal{B}_{0} \cup\{e\}$ é gerado por $\mathcal{B}_{0}$, temos que $\mathcal{B}_{0}$ gera $\Gamma$. Além disso, temos que $\mathrm{L}_{\mathcal{B}}(\omega) \leq j$. Logo, pela desigualdade da esquerda da equação (3.23), obtemos

$$
\mathrm{L}_{\mathcal{B}_{0}}(\omega) \leq j \leq \frac{1}{R} d\left(x_{0}, \omega\left(x_{0}\right)\right)+1 .
$$

Logo a afirmação está provada.

Observação 3.7.2 Até aqui a prova é seguida, em grande parte, de [dlH00] (Teorema 23). Algumas das equaçôes apresentadas até agora tem uma pequena diferença das que são dadas no teorema citado.

Agora seja $x$ em $M$, mas distinto de $x_{0}$. Definindo $\mathcal{B}_{x}$, analogamente como fizemos com $\mathcal{B}_{0}$, (vide $(3.20))$, i.e.,

$$
\mathcal{B}_{x}:=\left\{\omega \in \Gamma: \omega \neq \text { e e } \omega\left(\overline{B_{R}(x)}\right) \cap \overline{B_{R}(x)} \neq \varnothing\right\}
$$


obtemos que $\mathcal{B}_{x}$ gera $\Gamma$. Agora definamos

$$
\tilde{\mathcal{B}}_{x}:=\left\{\omega \in \Gamma: \omega \neq e \text { e } \omega\left(\overline{B_{2 R}(x)}\right) \cap \overline{B_{2 R}(x)} \neq \varnothing\right\}
$$

E observemos que $\tilde{\mathcal{B}}_{x}$ é finito, pois $\Gamma$ age propriamente sobre $M$.

Afirmação 4. $\tilde{\mathcal{B}}_{x}$ gera $\Gamma$.

Com efeito, é fácil ver que $\mathcal{B}_{x} \subset \tilde{\mathcal{B}}_{x}$, e dado que $\mathcal{B}_{x}$ gera $\Gamma$, então temos a afirmação.

Definamos $\tilde{r}_{x}$ da seguinte maneira

$$
\tilde{r}_{x}:=\inf \left\{d\left(\overline{B_{2 R}\left(x_{0}\right)}, \omega\left(\overline{B_{2 R}\left(x_{0}\right)}\right)\right): \omega \in \Gamma \backslash\left[\tilde{\mathcal{B}}_{x} \cup\{e\}\right]\right\}
$$

Agora, da mesma maneira como fizemos com $r_{0}$, vamos obter que

$$
\tilde{r}_{x}=\min \left\{d\left(\overline{B_{R}\left(x_{0}\right)}, \omega\left(\overline{B_{R}\left(x_{0}\right)}\right)\right): \omega \in \Gamma \backslash\left[\tilde{\mathcal{B}}_{x} \cup\{e\}\right]\right\}
$$

Além disso, também obtemos

$$
\mathrm{L}_{\tilde{\mathcal{B}}_{x}}(\omega) \leq \frac{1}{2 R} d(x, \omega(x))+1, \text { para todo } \omega \in \Gamma .
$$

Afirmação 5. Existe $\tilde{\omega} \in \Gamma$ tal que $\overline{B_{R}\left(x_{0}\right)} \subset \tilde{\omega}\left(\overline{\left.B_{2 R}(x)\right)}=\overline{B_{2 R}(\tilde{\omega}(x))}\right.$.

Primeiro lembremos que $M=\bigcup_{\omega \in \Gamma} \omega\left(\overline{B_{R}\left(x_{0}\right)}\right)$. Logo existe $\tilde{\omega} \in \Gamma$ tal que $\tilde{\omega}(x) \in \overline{B_{R}\left(x_{0}\right)}$. Dado $y \in \overline{B_{R}\left(x_{0}\right)}$, temos que

$$
d(\tilde{\omega}(x), y) \leq d\left(\tilde{\omega}(x), x_{0}\right)+d\left(x_{0}, y\right) \leq R+R=2 R .
$$

Logo temos a afirmação.

Afirmação 6. $\mathcal{B}_{0} \subset \tilde{\mathcal{B}}_{x}$.

Vamos provar a afirmação pelo absurdo, i.e., supomos que existe $\omega \in \mathcal{B}_{0}$ e $\omega \notin \tilde{\mathcal{B}}_{x}$. Portanto $\omega\left(\overline{B_{2 R}(x)}\right) \cap \overline{B_{2 R}(x)}=\varnothing$. Pela afirmação anterior, sabemos que existe $\tilde{\omega} \in \Gamma$ tal que $\tilde{\omega}(x) \epsilon$ $\overline{B_{R}\left(x_{0}\right)}$ e $\overline{B_{R}\left(x_{0}\right)} \subset \tilde{\omega}\left(\overline{B_{2 R}(x)}\right)=\overline{B_{2 R}(\tilde{\omega}(x))}$. 
Agora,

$$
\begin{array}{rlrl}
\varnothing=\omega\left(\overline{B_{2 R}(x)}\right) \cap \overline{B_{2 R}(x)} & =\tilde{\omega}\left(\omega\left(\overline{B_{2 R}(x)}\right) \cap \overline{B_{2 R}(x)}\right) & \\
& =\tilde{\omega} \omega\left(\overline{B_{2 R}(x)}\right) \cap \tilde{\omega}\left(\overline{B_{2 R}(x)}\right) & \\
& =\omega \tilde{\omega}\left(\overline{B_{2 R}(x)}\right) \cap \tilde{\omega}\left(\overline{B_{2 R}(x)}\right) & & \text { dado que } \Gamma \text { é abeliano } \\
& =\omega\left(\overline{B_{2 R}(\tilde{\omega}(x))}\right) \cap \overline{B_{2 R}(\tilde{\omega}(x))} & \text { pois } \tilde{\omega} \text { é uma isometria. }
\end{array}
$$

Portanto $\omega\left(\overline{B_{2 R}(\tilde{\omega}(x))}\right) \cap \overline{B_{2 R}(\tilde{\omega}(x))}=\varnothing$. Mas isso é um absurdo, pois $\overline{B_{R}\left(x_{0}\right)} \subset \tilde{\omega}\left(\overline{\left.B_{2 R}(x)\right)}\right.$ e $\omega\left(\overline{B_{R}\left(x_{0}\right)}\right) \cap \overline{B_{R}\left(x_{0}\right)} \neq \varnothing$, dado que $\omega \in \mathcal{B}_{0}$. Portanto $\mathcal{B}_{0} \subset \tilde{\mathcal{B}}_{x}$. E assim temos a prova da afirmação.

Observação 3.7.3 Observe que se y é um ponto em $M$, distinto de $x$ e $x_{0}$, então temos que

$$
\tilde{\mathcal{B}}_{y}:=\left\{\omega \in \Gamma: \omega \neq e \quad e \quad \omega\left(\overline{B_{2 R}(x)}\right) \cap \overline{B_{2 R}(x)} \neq \varnothing\right\}
$$

gera $\Gamma$ e $\mathcal{B}_{0} \subset \tilde{\mathcal{B}}_{y}$.

Afirmação 7. Para todo $\omega \in \tilde{\mathcal{B}}_{x} \backslash\left[\mathcal{B}_{0} \cup\{e\}\right]$ temos que

$$
R<d\left(x_{0}, \omega\left(x_{0}\right)\right) \leq 6 R
$$

Seja $\omega \in \tilde{\mathcal{B}}_{x} \backslash\left[\mathcal{B}_{0} \cup\{e\}\right]$, então temos que $R<d\left(x_{0}, \omega\left(x_{0}\right)\right)$. Por outro lado, dado que existe $\tilde{\omega} \in \Gamma$ tal que $\tilde{\omega}(x) \in \overline{B_{R}\left(x_{0}\right)}$, segue-se que

$$
d\left(x_{0}, \omega\left(x_{0}\right)\right) \leq d\left(x_{0}, \tilde{\omega}(x)\right)+d(\tilde{\omega}(x), \omega \tilde{\omega}(x))+d\left(\omega \tilde{\omega}(x), \omega\left(x_{0}\right)\right) .
$$

Agora,

$$
d\left(x_{0}, \tilde{\omega}(x)\right) \leq R, \text { dado que } \tilde{\omega}(x) \in B_{R}\left(x_{0}\right) .
$$

Também temos que

$$
d\left(\omega \tilde{\omega}(x), \omega\left(x_{0}\right)\right)=d\left(\tilde{\omega}(x), x_{0}\right) \leq R, \text { dado que } \tilde{\omega}(x) \in \overline{B_{R}\left(x_{0}\right)} .
$$


Por outro lado, seja $y \in \omega\left(\overline{B_{2 R}(x)}\right) \cap \overline{B_{2 R}(x)} \neq \varnothing$, pois $\omega \in \tilde{\mathcal{B}}_{x}$. Agora

$$
\begin{aligned}
d(\tilde{\omega}(x), \omega \tilde{\omega}(x)) & =d(\tilde{\omega}(x), \tilde{\omega} \omega(x)) & & \text { pois } \Gamma \text { é abeliano } \\
& =d(x, \omega(x)) & & \\
& \leq d(x, y)+d(y, \omega(x)) & & \text { por desigualdade triangular } \\
& \leq 2 R+2 R & & \text { pois } y \in \omega\left(\overline{B_{2 R}(x)}\right) \cap \overline{B_{2 R}(x)} .
\end{aligned}
$$

Portanto, aplicando (3.27), (3.28) e (3.29) na equação (3.26), temos que $d\left(x_{0}, \omega\left(x_{0}\right)\right) \leq 6 R$. Logo $R<d\left(x_{0}, \omega\left(x_{0}\right)\right) \leq 6 R$, para todo $\omega \in \tilde{\mathcal{B}}_{x} \backslash\left[\mathcal{B}_{0} \cup\{e\}\right]$

Observação 3.7.4 Observe que se y é um ponto em $M$, distinto de $x$ e $x_{0}$, e dado $\mathcal{B}_{0} \subset \tilde{\mathcal{B}}_{y}$, então

$$
R<d\left(x_{0}, \omega\left(x_{0}\right)\right) \leq 6 R, \text { para todo } \omega \in \tilde{\mathcal{B}}_{y} \backslash\left[\mathcal{B}_{0} \cup\{e\}\right]
$$

Até o momento provamos que $\mathcal{B}_{0}$ e $\tilde{\mathcal{B}}_{x}$ são finitos e geram $\Gamma$. Assim, pelo Lema 3.7.1, existe $\lambda \geq 1$ tal que

$$
\lambda^{-1} \mathrm{~L}_{\mathcal{B}_{0}}(\bar{\omega}) \leq \mathrm{L}_{\tilde{\mathcal{B}}_{x}}(\bar{\omega}) \leq \lambda \mathrm{L}_{\mathcal{B}_{0}}(\bar{\omega}) \text {, para todo } \bar{\omega} \in \Gamma
$$

Onde $\lambda=\max \left\{\lambda_{1}, \lambda_{2}\right\}, \quad \lambda_{1}=\max \left\{\mathrm{L}_{\tilde{\mathcal{B}}_{x}}(\omega): \omega \in \mathcal{B}_{0}\right\}$ e $\lambda_{2}=\max \left\{\mathrm{L}_{\mathcal{B}_{o}}(\omega): \omega \in \tilde{\mathcal{B}}_{x}\right\}$.

Observemos que neste caso $\lambda_{1}=1$ e $\lambda_{2}=\max \left\{\mathrm{L}_{\mathcal{B}_{o}}(\omega): \omega \in \tilde{\mathcal{B}}_{x} \backslash\left[\mathcal{B}_{0} \cup\{e\}\right]\right\} \geq 1$, e portanto $\lambda=\lambda_{2}$.

Afirmação 8. Existe $N$ em $\mathbb{N}$ tal que $\lambda_{2} \leq N$.

Primeiro lembremos que, pela Afirmação $2, \frac{r_{0}}{2} \leq R$. Seja $\omega \in \tilde{\mathcal{B}}_{x} \backslash\left[\mathcal{B}_{0} \cup\{e\}\right]$. Agora, na prova da Afirmação 3 , obtimos que dado $\omega \notin \mathcal{B}_{0} \cup\{e\}$, existe $j \in \mathbb{N}$, com $j \geq 3$, tal que (vide equação (3.23))

$$
R(j-1) \leq d\left(\omega\left(x_{0}\right), x_{0}\right)<R j
$$

Além disso, também temos que (vide a equação (3.24))

$$
\mathrm{L}_{\mathcal{B}_{0}}(\omega) \leq j \leq \frac{1}{R} d\left(x_{0}, \omega\left(x_{0}\right)\right)+1
$$

Agora, $\frac{r_{0}}{2} \leq R<d\left(x_{0}, \omega\left(x_{0}\right)\right)$. Assim, por propriedade Arquimediana dos números, existem $k$ e $J \in \mathbb{N}$, tais que

$$
(k-1) \frac{r_{0}}{2} \leq d\left(x_{0}, \omega\left(x_{0}\right)\right)<k \frac{r_{0}}{2}
$$


e

$$
J \frac{r_{0}}{2} \leq 6 R<(J+1) \frac{r_{0}}{2}
$$

Observemos que $k \geq 3$ e $J \geq 6$, pois $2 r_{0}<r_{0}+2 R \leq d\left(x_{0}, \omega\left(x_{0}\right)\right)$ e $\frac{r_{0}}{2} \leq R$.

Afirmamos que $k \geq j$. Para provar isto, vamos supor que não acontece, i.e., supomos que $k<j$. Portanto $k+1 \leq j$. Logo, pelas equações (3.31) e (3.33), vamos ter que

$$
k R \leq(j-1) R \leq d\left(\omega\left(x_{0}\right), x_{0}\right)<k \frac{r_{0}}{2} .
$$

Logo $k R<k \frac{r_{0}}{2}$. Mas isso é um absurdo, pois $\frac{r_{0}}{2} \leq R$. Logo $k \geq j$.

Agora, afirmamos que $k<J+2$. Para provar isto, vamos supor pelo absurdo, i.e., supomos que $k \geq J+2$. Assim, pelas equações (3.31) e (3.33), temos

$$
6 R<(J+1) \frac{r_{0}}{2} \leq(k-1) \frac{r_{0}}{2} \leq d\left(x_{0}, \omega\left(x_{0}\right)\right) .
$$

Então $6 R<d\left(x_{0}, \omega\left(x_{0}\right)\right)$. Mas isso é um absurdo, pois contradiz a Afirmação 7 . Logo $k<J+2$. Assim, podemos concluir que $j \leq k \leq J+1$. Logo, pela equação (3.32), temos que

$$
\mathrm{L}_{\mathcal{B}_{0}}(\omega) \leq j \leq J+1, \quad \text { para todo } \omega \in \tilde{\mathcal{B}}_{x} \backslash\left[\mathcal{B}_{0} \cup\{e\}\right] .
$$

Definindo $N:=J+1$, e dado que $\omega$ é qualquer elemento em $\tilde{\mathcal{B}}_{x} \backslash\left[\mathcal{B}_{0} \cup\{e\}\right]$, temos que $\lambda=\lambda_{2} \leq N$. E assim a afirmação está provada.

Observação 3.7.5 Observe que $J$ não depende do ponto $x$, i.e., se y é outro ponto em $M$, distinto de $x$ e $x_{0}$, e definimos $\tilde{\mathcal{B}}_{y}$ de forma análoga como fizemos com $\tilde{\mathcal{B}}_{x}$, então obtemos que $\mathcal{B}_{0} \subset \tilde{\mathcal{B}}_{y}$ e $\mathrm{L}_{\mathcal{B}_{0}}(\omega) \leq J+1$, para todo $\omega \in \tilde{\mathcal{B}}_{y}$.

Agora, pela equação (3.30) e pela Afirmação 8, vamos ter que

$$
\frac{1}{N} \mathrm{~L}_{\mathcal{B}_{0}}(\bar{\omega}) \leq \mathrm{L}_{\tilde{\mathcal{B}}_{x}}(\bar{\omega}) \leq N \mathrm{~L}_{\mathcal{B}_{0}}(\bar{\omega}) \text {, para cada } \bar{\omega} \in \Gamma \text {. }
$$

A equação (3.25) diz que

$$
\mathrm{L}_{\tilde{\mathcal{B}}_{x}}(\bar{\omega}) \leq \frac{1}{2 R} d(x, \bar{\omega}(x))+1 \text {, para todo } \bar{\omega} \in \Gamma .
$$


Portanto, aplicando a equação (3.35) na equação (3.36), obtemos

$$
\frac{1}{N} \mathrm{~L}_{\mathcal{B}_{0}}(\bar{\omega}) \leq \mathrm{L}_{\tilde{\mathcal{B}}_{x}}(\bar{\omega}) \leq \frac{1}{2 R} d(x, \bar{\omega}(x))+1 \text {, para todo } \bar{\omega} \in \Gamma .
$$

Pela observação 3.7.5, obtemos que os dois extremos da equação (3.37) não dependem do ponto $x$. Portanto

$$
\frac{1}{N} \mathrm{~L}_{\mathcal{B}_{0}}(\bar{\omega}) \leq \frac{1}{2 R} d(\cdot, \bar{\omega}(\cdot))+1, \text { para todo } \bar{\omega} \in \Gamma
$$

Dado que $\mathcal{A}$ e $\mathcal{B}_{0}$ geram $\Gamma$, temos, pelo Lema 3.7.1, que existe $\bar{\lambda} \geq 1$, tal que

$$
\bar{\lambda}^{-1} \mathrm{~L}_{\mathcal{A}}(\bar{\omega}) \leq \mathrm{L}_{\mathcal{B}_{0}}(\bar{\omega}) \leq \bar{\lambda} \mathrm{L}_{\mathcal{A}}(\bar{\omega}), \text { para todo } \bar{\omega} \in \Gamma
$$

Portanto

$$
\frac{\bar{\lambda}^{-1}}{N} \mathrm{~L}_{\mathcal{A}}(\bar{\omega}) \leq \frac{1}{N} \mathrm{~L}_{\mathcal{B}_{0}}(\bar{\omega}) \leq \frac{1}{2 R} d(\cdot, \bar{\omega}(\cdot))+1 \text {, para todo } \bar{\omega} \in \Gamma
$$

Assim, definindo $P:=\frac{\bar{\lambda}^{-1}}{N}$ e $r:=2 R$, obtemos que

$$
P \mathrm{~L}_{\mathcal{A}}(\bar{\omega}) \leq \frac{1}{r} d(\cdot, \bar{\omega}(\cdot))+1, \text { para todo } \bar{\omega} \in \Gamma .
$$

E assim temos a primeira parte da Proposição.

Observe que, dado que $X$ é compacto e que $\Gamma$ age livremente, cada elemento $\omega \in \Gamma$ é uma isometria de tipo hiperbólico (vide definição 2.2.3). Em particular, cada $\omega_{i}=v_{1}+v_{2}+\cdots+v_{k-1}+i v_{k}$ é de tipo hiperbólico. Seja, assim, $x_{i} \in \operatorname{Min}\left(\omega_{i}\right)$. Então, pela equação (3.40), obtemos que

$$
P \mathrm{~L}_{\mathcal{A}}\left(\omega_{i}\right) \leq \frac{1}{r} d\left(x_{i}, \omega_{i}\left(x_{i}\right)\right)+1=\frac{1}{r}\left|\omega_{i}\right|+1 .
$$

Agora, dado que $r$ e $P$ são constantes positivas e $\mathrm{L}_{\mathcal{A}}\left(\omega_{i}\right)=i+k-1$, temos que a sequência $\left\{\left|\omega_{i}\right|\right\}_{i \geq 1}$ é não limitada. Portanto temos a prova da segunda parte da proposição.

Observação 3.7.6 Observe que na prova da Proposição 3.7.1, quando desejávamos provar a equação (3.17), nunca usamos os fatos de que $\mathcal{A}$ fosse uma base para $\Gamma$ e que $\Gamma$ age livremente sobre $M$, somente que $\mathcal{A}$ gera $\Gamma$ e que $\Gamma$ é abeliano. Portanto, supondo que $X=M / \Gamma$ é um good orbifold compacto, onde $\Gamma$ é abeliano, então, se $\mathcal{B} \subset \Gamma$ gera $\Gamma$ (onde, pela Proposição 3.5 .1 e pelo item número 5 da Proposição 2.2.1, podemos supor que $\mathcal{B}$ é finito), temos que existem constantes positivas 
Per, tais que

$$
P \mathrm{~L}_{\mathcal{B}}(\omega) \leq \frac{1}{r}|\omega|+1 \text {, para todo } \omega \in \Gamma \text {. }
$$

Proposição 3.7.2 Seja $X=M / \Gamma$ um good orbifold Riemanniano compacto, onde $M$ é uma variedade Riemanniana tal que $\operatorname{dim}(M)>1$ e $\Gamma=\tilde{\Gamma} \ltimes \mathbb{Z}^{k}$, com $k \geq 2$ e $\tilde{\Gamma}$ é um grupo finito. Então temos que o orbifold Riemanniano $X=M / \Gamma$ admite uma família $\left\{\left(\omega_{i},\left.\alpha_{i}\right|_{[0,1]}\right)\right\}_{i \in \mathbb{N}}$ de geodésicas fechadas geometricamente distintas. Além disso, se $\left\{\left(\bar{\omega}_{i},\left.\alpha_{i}\right|_{\left[0, t_{i}\right]}\right)\right\}_{i \in \mathbb{N}}$ é uma família de geodésicas fechadas primitivas da família $\left\{\left(\omega_{i},\left.\alpha_{i}\right|_{[0,1]}\right)\right\}_{i \in \mathbb{N}}$, então existem constantes positivas $\bar{P}$ e $r$, tais que

$$
\bar{P}_{\mathrm{L}_{\mathcal{A}}}\left(\omega_{i}\right) \leq \frac{1}{r}\left|\bar{\omega}_{i}\right|+1, \text { para cada } i \in \mathbb{N}
$$

$e$

$$
\bar{P} \mathrm{~L}_{\mathcal{A}}\left(\omega_{i}\right) \leq \frac{1}{r} \mathcal{L}_{X}\left(\left.\alpha_{i}\right|_{[0,1]}\right)+1 \text {, para cada } i \in \mathbb{N}
$$

onde $\mathcal{A}$ é como na Proposição 3.7.1.

Em particular, $\left\{\left|\bar{\omega}_{i}\right|\right\}_{i \in \mathbb{N}}, \quad\left\{\left|\omega_{i}\right|\right\}_{i \in \mathbb{N}}$ e $\left\{\mathcal{L}_{X}\left(\left.\alpha_{i}\right|_{[0,1]}\right)\right\}_{i \in \mathbb{N}}$ são sequências reais não limitadas.

Demonstração. Na prova da primeira parte do Teorema 3.6.1 construimos uma sequência

$$
\left\{\left(\omega_{i},\left.\alpha_{i}\right|_{[0,1]}\right)\right\}_{i \in \mathbb{N}}
$$

de geodésicas fechadas em $X$ apartir de uma base $\mathcal{A}=\left\{v_{1}, \ldots, v_{k}\right\}$ de $\mathbb{Z}^{k}$ e onde $\omega_{i}:=v_{1}+v_{2}+$ $\cdots+v_{k-1}+i v_{k}$ e $\mathcal{L}\left(\left.\alpha_{i}\right|_{[0,1]}\right)=\left|\omega_{i}\right|$ (vide a prova do Teorema 3.6.1). Agora, dado que $\mathbb{Z}^{k}$ é de índice finito em $\Gamma$, temos, pelo Teorema 3.4.1, que existe uma subsequência $\left\{\left(\omega_{i_{k}},\left.\alpha_{i_{k}}\right|_{[0,1]}\right)\right\}_{k \in \mathbb{N}}$ de $\left\{\left(\omega_{i},\left.\alpha_{i}\right|_{[0,1]}\right)\right\}_{i \in \mathbb{N}}$, onde todas geodésicas fechadas de $\left\{\left(\omega_{i_{k}},\left.\alpha_{i_{k}}\right|_{[0,1]}\right)\right\}_{k \in \mathbb{N}}$ são geometricamente distintas em $X$. Logo temos a primeira parte da proposição.

Agora vamos provar a segunda parte da proposicão. Seja $\left\{\left(\bar{\omega}_{i},\left.\alpha_{i}\right|_{\left[0, t_{i}\right]}\right)\right\}_{i \in \mathbb{N}}$ é uma família de geodésicas fechadas primitivas de $\left\{\left(\omega_{i},\left.\alpha_{i}\right|_{[0,1]}\right)\right\}_{i \in \mathbb{N}}$. Agora, pela Proposição 3.4.4, cada $t_{i}=\frac{1}{m_{i}}$, onde cada $m_{i} \in \mathbb{N}$. Por outro lado, pelo item número 1 da Proposição 3.4.5, temos que para cada $i \in \mathbb{N}$, existe $k_{i} \in \mathbb{N}$ tal que $\bar{\omega}_{i}^{k_{i} m_{i}}=\omega_{i}^{{ }_{i}}$. Então, pela equação (3.17), temos

$$
P \mathrm{~L}_{\mathcal{A}}\left(\omega_{i}^{k_{i}}\right) \leq \frac{1}{r}\left|\omega_{i}^{k_{i}}\right|+1=\frac{1}{r}\left|\bar{\omega}_{i}^{k_{i} m_{i}}\right|+1 \text {, para cada } i \in \mathbb{N} \text {. }
$$


Dado que $\mathcal{A}=\left\{v_{1}, v_{2}, \ldots, v_{k-1}, v_{k}\right\}$ gera $\mathbb{Z}^{k}$ e $\omega_{i}:=v_{1}+, v_{2}+\cdots+v_{k-1}+i v_{k}$, então, aplicando isto na equação (3.44), obtemos que

$k_{i} P(i+k-1)=k_{i} P \mathrm{~L}_{\mathcal{A}}\left(\omega_{i}\right)=P \mathrm{~L}_{\mathcal{A}}\left(\omega_{i}^{k_{i}}\right) \leq \frac{1}{r}\left|\omega_{i}^{k_{i}}\right|+1=\frac{1}{r}\left|\bar{\omega}_{i}^{k_{i} m_{i}}\right|+1$, para cada $i \in \mathbb{N}$.

Agora afirmamos que $\left|\bar{\omega}_{i}^{k_{i} m_{i}}\right| \leq k_{i} m_{i}\left|\bar{\omega}_{i}\right|$. Antes de provar a afirmação, observemos que, pelo item 3 da Proposição 2.2.1, todos os elementos de $\Gamma$ são semi-simples. Assim, seja $x_{i} \in \operatorname{Min}\left(\bar{\omega}_{i}\right)$. Portanto, pela definição de $\left|\bar{\omega}_{i}^{m m_{i}}\right|$ (vide a definição 2.2.2), temos que $\left|\bar{\omega}_{i}^{m m_{i}}\right| \leq d\left(x_{i}, \bar{\omega}_{i}^{m m_{i}}\left(x_{i}\right)\right)$. Então, usando a desigualdade triangular, obtemos

$$
\left|\bar{\omega}_{i}^{k_{i} m_{i}}\right| \leq d\left(x_{i}, \bar{\omega}_{i}^{k_{i} m_{i}}\left(x_{i}\right)\right) \leq k_{i} m_{i} d\left(x_{i}, \bar{\omega}_{i}\left(x_{i}\right)\right)=k_{i} m_{i}\left|\bar{\omega}_{i}\right|
$$

Logo temos a prova da afirmação.

Agora, aplicando a afirmação anterior na equação (3.45), obtemos

$$
k_{i} P(i+k-1) \leq \frac{1}{r} k_{i} m_{i}\left|\bar{\omega}_{i}\right|+1 \text {, para cada } i \in \mathbb{N} .
$$

Dado que $k_{i} m_{i} \geq 1$, temos

$$
\frac{1}{m_{i}} P(i+k-1) \leq \frac{1}{r}\left|\bar{\omega}_{i}\right|+\frac{1}{m m_{i}} \leq \frac{1}{r}\left|\bar{\omega}_{i}\right|+1 \text {, para cada } i \in \mathbb{N} .
$$

Por outro lado, na prova do Corolário 1 obtimos que existe $l \in \mathbb{N}$, tal que $\frac{1}{l} \leq t_{i}=\frac{1}{m_{i}}$, para cada $i \in \mathbb{N}$ (vide equação (3.5)). Assim

$$
\frac{1}{l} P(i+k-1) \leq \frac{1}{r}\left|\bar{\omega}_{i}\right|+1, \text { para cada } i \in \mathbb{N} .
$$

Logo, definindo $\bar{P}:=\frac{P}{l}$, obtemos que

$$
\bar{P} \mathrm{~L}_{\mathcal{A}}\left(\omega_{i}\right)=\bar{P}(i+k-1) \leq \frac{1}{r}\left|\bar{\omega}_{i}\right|+1 \text {, para cada } i \in \mathbb{N} .
$$

Então temos a primera equação que desejávamos.

Dado que para cada $i \in \mathbb{N}$ temos que $\left(\bar{\omega}_{i},\left.\alpha_{i}\right|_{\left[0, t_{i}\right]}\right)$ é uma geodésica fechada, então

$$
\left|\bar{\omega}_{i}\right| \leq \mathcal{L}\left(\left.\alpha_{i}\right|_{\left[0, t_{i}\right]}\right)=: \mathcal{L}_{X}\left(\left.\alpha_{i}\right|_{[0,1]}\right)
$$


Assim, aplicando (3.50) em (3.49), obtemos

$$
\bar{P} \mathrm{~L}_{\mathcal{A}}\left(\omega_{i}\right)=\bar{P}(i+k-1) \leq \frac{1}{r} \mathcal{L}_{X}\left(\left.\alpha_{i}\right|_{[0,1]}\right)+1 \text {, para cada } i \in \mathbb{N} .
$$

Portanto temos a segunda equação desejada.

Dado que $\bar{P}$ e $r$ são constantes positivas e usando as equações (3.49) e (3.50), temos que as sequências reais $\left\{\left|\bar{\omega}_{i}\right|\right\}_{i \in \mathbb{N}}$ e $\left\{\mathcal{L}_{X}\left(\left.\alpha_{i}\right|_{[0,1]}\right)\right\}_{i \in \mathbb{N}}$ são não limitadas, e dado que $\left|\omega_{i}\right|=\mathcal{L}_{X}\left(\left.\alpha_{i}\right|_{[0,1]}\right)$, então $\left\{\left|\omega_{i}\right|\right\}_{i \in \mathbb{N}}$ também é não limitada. Assim temos a prova completa da proposição.

Proposição 3.7.3 Seja $X=M / \Gamma$ um good orbifold, e supomos que existe um subgrupo $\Gamma_{0}$ de $\Gamma$ que é normal e de indice finito. Definamos $X_{0}:=M / \Gamma_{0}$ e seja $\left(\omega,\left.\alpha\right|_{[0,1]}\right)$ uma geodésica fechada primitiva em $X_{0}$. Se $\left(\omega_{0},\left.\alpha\right|_{\left[0, t_{0}\right]}\right)$ é uma geodésica primitiva de $\left(\omega,\left.\alpha\right|_{[0,1]}\right)$ e $X$ e $k$ é o índice de $\Gamma_{0}$ em $\Gamma$, então

$$
\mathcal{L}\left(\left.\alpha\right|_{[0,1]}\right) \leq k \mathcal{L}_{X}\left(\left.\alpha\right|_{[0,1]}\right)=k \mathcal{L}\left(\left.\alpha\right|_{\left[0, t_{0}\right]}\right)
$$

$e \frac{1}{k} \leq t_{0}$

Demonstração. Primero observemos que se $\omega_{0} \in \Gamma_{0}$, então $t_{0}=1$, pois $\left(\omega,\left.\alpha\right|_{[0,1]}\right)$ uma geodésica fechada primitiva em $X_{0}$. Logo neste caso temos o resultado.

Agora vamos supor que $\omega_{0} \notin \Gamma_{0}$. Dado que $\left(\omega_{0},\left.\alpha\right|_{\left[0, t_{0}\right]}\right)$ é geodésica primitiva de $\left(\omega,\left.\alpha\right|_{[0,1]}\right)$, então $t_{0}=\frac{1}{n_{0}}$, onde $n_{0} \in \mathbb{N}$. Além disso, temos que

$$
\mathcal{L}\left(\left.\alpha\right|_{[0,1]}\right)=n_{0} \mathcal{L}\left(\left.\alpha\right|_{\left[0, t_{0}\right]}\right)
$$

Logo afirmamos que $n \leq k$. Vamos provar a afirmação pelo absurdo, i.e., vamos supor que $k<n$. Agora, dado que $k$ é o índice de $\Gamma_{0}$ e por hipótese $\Gamma_{0}$ é um subgrupo normal de $\Gamma$, temos que $\omega_{0}^{k} \in \Gamma_{0}$. Dado que $\left(\omega_{0},\left.\alpha\right|_{\left[0, t_{0}\right]}\right)$ é uma geodésica fechada e $\omega_{0}^{k} \in \Gamma_{0}$, então $\left(\omega_{0}^{k},\left.\alpha\right|_{\left[0, k t_{0}\right]}\right)$ é uma geodésica fechada em $X_{0}$ e $k t_{0}<1$, i.e, $\left(\omega,\left.\alpha\right|_{[0,1]}\right)$ não é uma geodésica fechada primitiva em $X_{0}$, que é um absurdo. Por tanto $n_{0} \leq k$ e $\frac{1}{k} \leq t_{0}$. Assim a prova da proposição.

Observação 3.7.7 Observemos que o Corolário 1 e as Proposições 3.6.1, 3.7.2 e 3.7.3 garantam a segunda conclusão do Teorema 3.6.1. Porém, as Proposições 3.6.1 e 3.7.2 dão uma informação adicional: dada a família $\left\{\left(\omega_{i},\left.\alpha_{i}\right|_{[0,1]}\right)\right\}_{i \in \mathbb{N}}$ de geodésicas fechadas geometricamente distintas em $M / \Gamma_{0}$ e a família $\left\{\left(\bar{\omega}_{i},\left.\alpha_{i}\right|_{\left[0, t_{i}\right]}\right)\right\}_{i \in \mathbb{N}}$ de geodésicas primitivas associada, temos que a sequência 
real $\left\{\left|\bar{\omega}_{i}\right|\right\}_{i \in \mathbb{N}}$ é não limitada.

Por outro lado, é importante dizer que nos Teorema 3.5.1 e 3.6.1 e na Proposição 3.7.2 usamos o Teorema 2.4.1 para construir a famılia infinita de geodésicas fechadas, mas é bom saber que também podemos usar o Teorema 2.3.1 para obter tal família; resultado que é baseado no método de duplo encurtamento (vide Capítulo 2, Seção 2.3). Agora, no Teorema 3.6.1 e na Proposição 3.7.2, temos resultados sobre os comprimentos em $X$ das geodésicas fechadas, e é bom observar que usando o Teorema 2.3.1 vamos garantir as mesmas conclusões sobre tais comprimentos.

\subsection{O caso do Triângulo Equilátero: uma prova alternativa}

Nesta seção daremos uma prova alternativa dos resultados principais do Capítulo 3 para o caso particular onde $M$ é o espaço Euclidiano $\mathbb{R}^{2}$ (com uma métrica não necessariamente euclidiana) e $\Gamma$ é um grupo de Coxeter Euclidiano. Como veremos na Proposição 3.8.1, neste caso particular poderemos inferir novas propriedades da família das infinitas geodésicas.

Vamos lembrar uma definição antes de dar nossa proposição.

Definição 3.8.1 Seja $\left\{H_{i}: i \in I\right\}$ uma família de linhas em $\mathbb{R}^{2}$. Um subgrupo $\Gamma$ de Iso $\left(\mathbb{R}^{2}\right)$ gerado pelo conjunto de reflexôes $\left\{r_{i}: i \in I\right\}$, onde $r_{i}$ é a reflexão na linha $H_{i}$, é um grupo de Coxeter se a topologia induzida em $\Gamma$ de Iso $\left(\mathbb{R}^{2}\right)$ é discreto e age sobre $\mathbb{R}^{2}$ propriamente. Um grupo de Coxeter infinito também é chamado um grupo de Weyl afim.

Nesta seção estamos interessados em considerar a seguinte família de retas (vide figura 3.8 ) :

1. Seja $H_{1}^{n}$ a família de retas paralelas ao eixo $x$ de $\mathbb{R}^{2}$;

2. Seja $H_{2}^{n}$ a família de retas que vão formar um ângulo de 60 graus com as retas $H_{1}^{n}$;

3. Seja $H_{3}^{n}$ a família de retas que vão formar um ângulo de 60 graus com as retas $H_{1}^{n}$ e com as $\operatorname{retas} H_{2}^{n}$. 


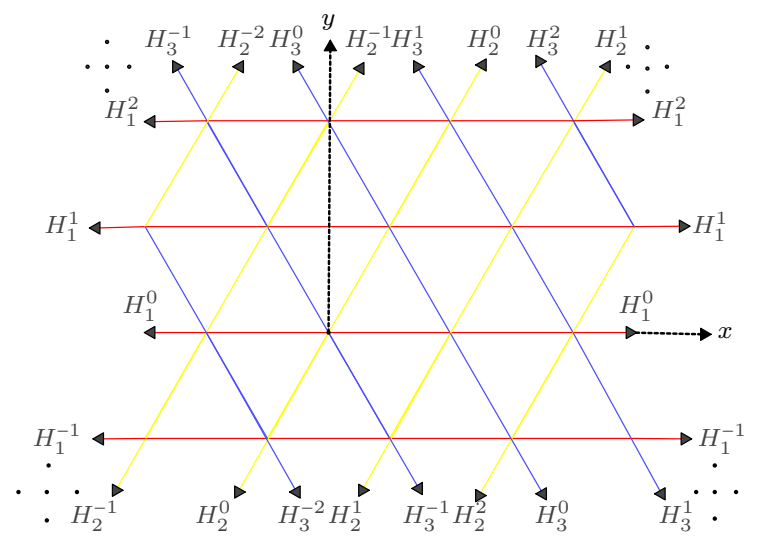

Figura 3.8

Proposição 3.8.1 Seja $M=\left(\mathbb{R}^{2}, \mathrm{~g}\right)$ e $\Gamma$ o grupo de Coxeter euclidano descrito acima. Suponha que $\Gamma$ seja um subgrupo de isometrias de Iso $(M, \mathrm{~g})$. Então

1. M/ $\Gamma$ tem infinitas geodésicas geometricamente distintas;

2. tais geodésicas possuem pontos singulares isolados;

3. tais geodésicas não possuem primitivas distintas delas mesmas.

Observação 3.8.1 Utilizando o seção 3.3 ou Corolário 1 observa-se que o comprimento de tais geodésicas é não limitada.

A proposição será demonstrada ao longo de vários passos. Em particular o item (1) seguirá da Afirmação 2 e o item (3) seguirá da Afirmação 8.

Demonstração. Não é difícil ver que $\Gamma$ é finitamente gerado e $\Gamma$ é gerado pelas reflexões $r_{1}, r_{2}, r_{3}$ (vide [BH99] - Teorema 8.10), onde $r_{i}$ é a reflexão na linha $H_{i}^{0}$, para $i=1,2,3$, respectivamente. Agora definamos $e_{2}, v_{1}$ e $v_{2}$ os vetores com coordenadas $(0,1),(1,0)$ e $\left(\frac{1}{2}, \frac{\sqrt{3}}{2}\right)$, respectivamente. Assim

$$
\begin{aligned}
& r_{1}(x)=x-2\left\langle e_{2} ; x\right\rangle e_{2} ; \\
& r_{2}(x)=x-\frac{2}{3}\left\langle v_{2}-2 v_{1} ; x\right\rangle\left(v_{2}-2 v_{1}\right) \\
& r_{3}(x)=x-\frac{2}{3}\left\langle v_{1}+v_{1} ; x-v_{1}\right\rangle\left(v_{1}+v_{2}\right) .
\end{aligned}
$$

Definamos $\omega_{(1)}:=\left(r_{2} r_{1} r_{3}\right)^{2}$ e $\omega_{(2)}:=r_{2} r_{1} r_{2} r_{3}$. Fazendo os cálculos respectivos, obtemos que

$$
\begin{aligned}
& \omega_{(1)}(x)=x+3 v_{1}, \text { para todo } x \in \mathbb{R}^{2} \\
& \omega_{(2)}(x)=x+v_{1}+v_{2}, \text { para todo } x \in \mathbb{R}^{2} .
\end{aligned}
$$

Portanto, obtemos que $\omega_{(1)}$ e $\omega_{(2)}$ são elementos de $\Gamma$ e, além disso, são translações de $\mathbb{R}^{2}$. Por hipótese, para cada $n \in \mathbb{Z}$, temos que $H_{1}^{n}, H_{2}^{n}, H_{3}^{n}$ são as retas paralelas às retas $H_{1}^{0}, H_{2}^{0}, H_{3}^{0}$, 
respectivamente. E denotaremos por $H_{i}:=H_{j}^{0}$ para $j=1,2,3$, respectivamente. Para cada $j=1,2,3$ e cada $n \in \mathbb{Z}$, definamos $r_{(j, n)}$ como a reflexão na reta $H_{j}^{n}$. E portanto $r_{j}=r_{(j, 0)}$, para $j=1,2,3$, respectivamente. Agora, não é difícil observar que cada $n \in \mathbb{Z}$ satisfaz que $n\left(v_{1}+v_{2}\right) \in H_{1}^{n}$, $n v_{1} \in H_{2}^{n}$ e $(n+1) v_{1} \in H_{3}^{n}$. Assim

$$
\begin{aligned}
& r_{(1, n)}(x)=x-2\left\langle e_{2} ; x-n\left(v_{1}+v_{2}\right)\right\rangle e_{2} ; \\
& r_{(2, n)}(x)=x-\frac{2}{3}\left\langle v_{2}-2 v_{1} ; x-n v_{1}\right\rangle\left(v_{2}-2 v_{1}\right) ; \\
& r_{(3, n)}(x)=x-\frac{2}{3}\left\langle v_{1}+v_{1} ; x-(n+1) v_{1}\right\rangle\left(v_{1}+v_{2}\right) .
\end{aligned}
$$

Definamos $\mathcal{R}:=<r_{1}, r_{2}>$, i.e., $\mathcal{R}$ é o grupo gerado por $r_{1}$ e $r_{2}$. E seja $\Gamma_{0}:=<\omega_{(1)}, \omega_{(2)}>$, i.e., o grupo gerado por $\omega_{(1)}$ e $\omega_{(2)}$.

Afirmação 1. $\Gamma=\mathcal{R} \ltimes \Gamma_{0}$.

Primeiro observemos que $r_{3}=\omega_{2} r_{(3,-1)} \Gamma_{0} \cap \mathcal{R}=\{e\}$ e que $\Gamma_{0}$ é um subgrupo normal de $\Gamma$. Para provar esta afirmação, basta com observar que

$$
\begin{array}{llll}
\bullet r_{1}=r_{1} & \bullet r_{2}=r_{2} & \bullet r_{(3,-1)}=r_{1} r_{2} r_{1} & \bullet r_{1} r_{2}=r_{1} r_{2} \\
\bullet r_{2} r_{1}=r_{2} r_{1} & \bullet r_{1} r_{(3,0)}=r_{2} r_{1} & \bullet r_{2} r_{(3,0)}=\left(r_{2} r_{1}\right)^{2} & \bullet r_{(3,-1)} r_{1}=r_{1} r_{2} \\
\bullet r_{(3,0)} r_{2}=r_{2} r_{1} & \bullet r_{1} r_{2} r_{(3,0)}=r_{2} & \bullet r_{2} r_{1} r_{(3,-1)}=r_{1} & \bullet r_{1} r_{(3,-1)} r_{2}=r_{2} r_{1} r_{2} \\
\bullet r_{2} r_{(3,-1)} r_{1}=r_{2} r_{1} r_{2} & \bullet r_{(3,-1)} r_{1} r_{2}=r_{1} & \bullet r_{(3,-1)} r_{2} r_{1}=r_{2} &
\end{array}
$$

e que para cada par $m, k$ em $\mathbb{Z}$

$$
\begin{aligned}
& \text { - } r_{1} \omega_{(1)}^{k} \omega_{(2)}^{m}=\omega_{(1)}^{k} \omega_{(2)}^{-m} r_{1} \\
& \text { - } r_{2} \omega_{(1)}^{k} \omega_{(2)}^{m}=\omega_{(1)}^{-2 k-m} \omega_{(2)}^{3 k+2 m} r_{2} \\
& \text { - } r_{(3,-1)} \omega_{(1)}^{k} \omega_{(2)}^{m}=\omega_{(1)}^{k} \omega_{(2)}^{-m-3 k} r_{(3,-1)} \text {. }
\end{aligned}
$$

E assim está provada a Afirmação 1.

Para cada $i \in \mathbb{N}$, definamos $\omega_{i}:=\omega_{(2)} \omega_{(1)}^{i}$, i.e.,

$$
\omega_{i}(x)=x+\left(v_{1}+v_{2}\right)+3 i v_{1}, \text { para todo } x \in \mathbb{R}^{2}
$$

Afirmação 2. Para cada $n \in \mathbb{Z}$ e cada $i \in \mathbb{N}$, temos que $\omega_{i}$ não deixa invariante cada espaço afim $H_{1}^{n}$.

Vamos provar a afirmação pelo absurdo. Assim, dado que $n\left(v_{1}+v_{2}\right) \in H_{1}^{n}$, então $\omega_{i}\left(n\left(v_{1}+v_{2}\right)\right) \epsilon$ 
$H_{1}^{n}$. Portanto

$$
r_{(1, n)}\left(\omega_{i}\left(n\left(v_{1}+v_{2}\right)\right)\right)=\omega_{i}\left(n\left(v_{1}+v_{2}\right)\right) \text {. }
$$

Temos que

$$
\omega_{i}\left(n\left(v_{1}+v_{2}\right)\right)=n\left(v_{1}+v_{2}\right)+\left(v_{1}+v_{2}\right)+3 i v_{1}
$$

e

$$
r_{(1, n)}\left(\omega_{i}\left(n\left(v_{1}+v_{2}\right)\right)\right)=\omega_{i}\left(n\left(v_{1}+v_{2}\right)\right)-2\left\langle e_{2} ; \omega_{i}\left(n\left(v_{1}+v_{2}\right)\right)-n\left(v_{1}+v_{2}\right)\right\rangle e_{2} .
$$

Portanto

$$
\begin{aligned}
0 & =\left\langle e_{2} ; \omega_{i}\left(n\left(v_{1}+v_{2}\right)\right)-n\left(v_{1}+v_{2}\right)\right\rangle \\
& =\left\langle e_{2} ; v_{2}\right\rangle+\left\langle e_{2} ;(3 i+1) v_{1}\right\rangle \\
& =\left\langle e_{2} ; v_{2}\right\rangle \\
& =\frac{\sqrt{3}}{2} .
\end{aligned}
$$

Que é um absurdo. Logo temos a prova da afirmação.

Afirmação 3. Para cada $n \in \mathbb{Z}$ e cada $i \in \mathbb{N}$, temos que $\omega_{i}$ não deixa invariantes os espaços afim $H_{2}^{n}$.

Vamos provar a afirmação pelo absurdo. Assim, dado que $n v_{1} \in H_{1}^{n}$, então temos que $\omega_{i}\left(n v_{1}\right) \epsilon$ $H_{2}^{n}$. Portanto

$$
r_{(2, n)}\left(\omega_{i}\left(n v_{1}\right)\right)=\omega_{i}\left(n v_{1}\right)
$$

Agora

$$
\omega_{i}\left(n v_{1}\right)=n v_{1}+\left(v_{1}+v_{2}\right)+3 i v_{1}
$$

$\mathrm{e}$

$$
r_{(2, n)}\left(\omega_{i}\left(n v_{1}\right)\right)=\omega_{i}\left(n v_{1}\right)-\frac{2}{3}\left\langle v_{2}-2 v_{1} ; \omega_{i}\left(n v_{1}\right)-n v_{1}\right\rangle\left(v_{2}-2 v_{1}\right)
$$

Então

$$
\begin{aligned}
0 & =\left\langle v_{2}-2 v_{1} ; \omega_{i}\left(n v_{1}\right)-n v_{1}\right\rangle \\
& =\left\langle v_{2}-2 v_{1} ; v_{2}+(3 i+1) v_{1}\right\rangle \\
& =-3(3 i+1) .
\end{aligned}
$$

Mas isso é um absurdo, pois $i \in \mathbb{N}$. Portanto temos a afirmação.

Afirmação 4. Para cada $n \in \mathbb{Z}$ e cada $i \in \mathbb{N}$, temos que $\omega_{i}$ não deixa invariantes os espaços afim $H_{3}^{n}$. 
Vamos provar a afirmação pelo absurdo. Assim, dado que $n v_{1} \in H_{1}^{n}$, então $\omega_{i}\left((n+1) v_{1}\right) \in H_{3}^{n}$. Portanto

$$
r_{(3, n)}\left(\omega_{i}\left((n+1) v_{1}\right)\right)=\omega_{i}\left((n+1) v_{1}\right) .
$$

Temos que

$$
\omega_{i}\left((n+1) v_{1}\right)=(n+1) v_{1}+\left(v_{1}+v_{2}\right)+3 i v_{1}
$$

e

$$
r_{(3, n)}\left(\omega_{i}\left((n+1) v_{1}\right)=\omega_{i}\left((n+1) v_{1}-\frac{2}{3}\left\langle v_{1}+v_{2} ; \omega_{i}\left((n+1) v_{1}\right)-(n+1) v_{1}\right\rangle\left(v_{1}+v_{2}\right)\right.\right.
$$

Portanto

$$
\begin{aligned}
0 & =\left\langle v_{1}+v_{2} ; \omega_{i}\left((n+1) v_{1}\right)-(n+1) v_{1}\right\rangle \\
& =\left\langle v_{1}+v_{2} ; v_{2}+(3 i+1) v_{1}\right\rangle \\
& =3(3 i+1) .
\end{aligned}
$$

Mas isso é um absurdo, pois $i \in \mathbb{N}$. Logo vale a afirmação.

Afirmação 5. Para todo $\omega_{i}$ e todo $\omega \in \Gamma$ temos que $\omega \omega_{i} \omega^{-1}$ não deixa invariante nenhum estrato $H_{j}^{n}$.

Vamos supor que isto não acontece, i.e., vamos supor que existe $H_{j}^{n}$ qué é invariante por $\omega \omega_{i} \omega^{-1}$, para qualquer $\omega \in \Gamma$. Então temos que $\omega \omega_{i} \omega^{-1}\left(H_{j}^{n}\right)=H_{j}^{n}$. Logo $\omega_{i}\left(\omega^{-1}\left(H_{j}^{n}\right)\right)=\omega^{-1}\left(H_{j}^{n}\right)$. Portanto $\omega_{i}$ deixa invariante a $\omega^{-1}\left(H_{j}^{n}\right)$. Mas isso é um absurdo, pois contradiz as Afirmações 2,3 e 4 . Logo a afirmação está provada.

Afirmação 6. Se $\omega_{i} \neq \omega_{j}$, então não existe $\omega \in \Gamma$ tal que $\omega \omega_{i}^{m} \omega_{i}^{-1}=\omega_{j}^{k}$, para todo par $m, k \in \mathbb{Z}^{*}$. Sejam $\omega_{i} \neq \omega_{j}$ e suponhamos que existem $m, k \in \mathbb{Z}^{*}$ e $\omega \in \Gamma$ tais que $\omega \omega_{i}^{m} \omega_{i}^{-1}=\omega_{j}^{k}$. Observemos que $\omega_{i}^{m} \neq \omega_{j}^{k}$, pois $\left\{\omega_{(1)}, \omega_{(2)}\right\}$ é uma base para $\Gamma_{0}$. Agora, dado que $\omega \in \Gamma$ e pela Afirmação 1, temos que $\omega=\tilde{\omega} r$, onde $\tilde{\omega} \in \Gamma_{0}$ e $r \in \mathcal{R}$. Assim, $\omega \omega_{i}^{m} \omega_{i}^{-1}=\tilde{\omega} r \omega_{i}^{m} r^{-1} \tilde{\omega}^{-1}$. Seja $x \in \mathbb{R}^{2}$, então $r \omega_{i}^{m} r^{-1}(x)=r \omega_{i}^{m}\left(r^{-1}(x)\right)=r\left(r^{-1}(x)+m\left(v_{1}+v_{2}\right)+3 m i v_{1}\right)=x+r\left(m\left(v_{1}+v^{2}\right)+3 m i v_{1}\right)$

Logo $r \omega_{i}^{m} r^{-1}$ é um elemento de $\Gamma_{0}$. Portanto, dado que $\Gamma_{0}$ é abeliano, temos que

$$
\tilde{\omega} r \omega_{i}^{m} r^{-1} \tilde{\omega}^{-1}=\tilde{\omega} \tilde{\omega}^{-1} r \omega_{i}^{m} r^{-1}=r \omega_{i}^{m} r^{-1}
$$


Então $\omega_{j}^{k}=\omega \omega_{i}^{m} \omega^{-1}=r \omega_{i}^{m} r^{-1}$.

Por outro lado, $\mathcal{R}:=\left\{r_{1}, r_{1} r_{2}, r_{2} r_{1}, r_{1} r_{2} r_{1}, I\right\}$, onde $I$ é a isometria identidade. Observemos que o conjunto

$$
\mathfrak{R}:=\left\{x \in \mathbb{R}^{2}: x=c v_{1}+d v_{2} \text {, onde } c, d \in(0, \infty)\right\}
$$

é um domínio fundamental para a ação de $\mathcal{R}$ sobre $\mathbb{R}^{2}$. Assim, vamos provar a afirmação fazendo os distintos casos para $r$.

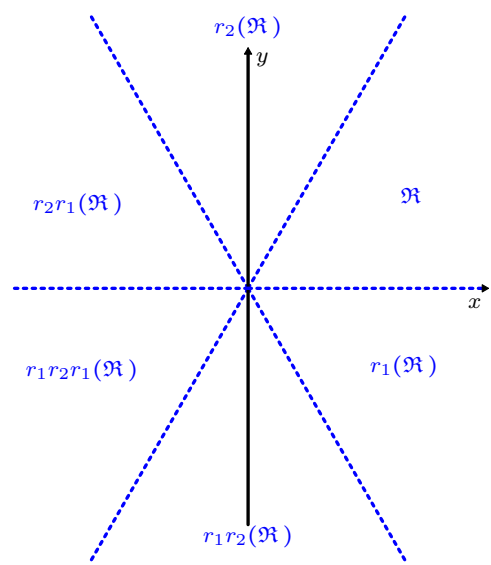

Figura 3.9: $O$ dominio fundamental $\mathfrak{R}$ e a ação de $\mathcal{R}$ sobre $\mathfrak{R}$.

1. Supomos que $m$ e $k$ são positivos. Neste caso é fácil observar que $\omega_{i}^{m}(0)$ e $\omega_{j}^{k}(0)$ estão em $\mathfrak{R}$. Além disso, para cada $r \in \mathcal{R}$, temos que $\omega_{i}^{m} r^{-1}(0)$ e $\omega_{r}^{k} r(0)$ estão em $\mathfrak{R}$. Assim, para cada $r \in \mathcal{R} \backslash\{I\}$ temos que $r \omega_{i}^{m} r^{-1}(0)$ não é um ponto em $\mathfrak{R}$. Portanto $r \omega_{i}^{m} r^{-1} \neq \omega_{j}^{k}$.

2. Se supomos que $m$ e $k$ são negativos, então, de maneira análoga ao raciocínio do item anterior, vamos obter que $r \omega_{i}^{m} r^{-1} \neq \omega_{j}^{k}$, para todo $r \in \mathcal{R}$.

3. Supomos que $m$ é negativo e $k$ é positivo. Neste caso $\omega_{i}^{m}(0)$ está em $r_{1} r_{2} r_{1}(\mathfrak{R})$ e $\omega_{j}^{k}(0)$ está em $\mathfrak{R}$. Agora, para todo $r \in \mathcal{R}$, temos que $\omega_{i}^{m} r(0) \in r_{1} r_{2} r_{1}(\mathfrak{R})$. Dado que estamos supondo que existe $r \in \mathcal{R}$ tal que $\omega_{j}^{k}=r \omega_{i}^{m} r^{-1}$, então $\omega_{j}^{k}(0)=r \omega_{i}^{m} r^{-1}(0)$. Logo $r$ tem que ser igual a $\left(r_{1} r_{2} r_{1}\right)^{-1}=r_{1} r_{2} r_{1}$. Assim, calculando, obtemos que

$$
r \omega_{i}^{m} r^{-1}(0)=r_{1} r_{2} r_{1} \omega_{i}^{m}(0)=r_{1} r_{2} r_{1}\left(m\left(v_{1}+v_{2}\right)+3 m i v_{1}\right)=-m(i+1)\left(v_{1}+v_{2}\right)+3 i v_{1} .
$$

Portanto

$$
\omega_{j}^{k}(0)=k\left(v_{1}+v_{2}\right)+3 j v_{1}=-m(i+1)\left(v_{1}+v_{2}\right)+3 i v_{1} .
$$

Assim, dado que $v_{1}+v_{2}$ e $v_{1}$ são linearmente independentes, temos que $k=-m$ e $i=j$. 
Mas isso é um absurdo pois, por hipótese, $\omega_{i} \neq \omega_{j}$. Logo $r \omega_{i}^{m} r^{-1} \neq \omega_{j}^{k}$, para todo $r \in \mathcal{R}$.

4. No caso no qual $m$ é positivo e $k$ é negativo, fazendo um raciocínio do item anterior, vamos obter que $r \omega_{i}^{m} r^{-1} \neq \omega_{j}^{k}$, para todo $r \in \mathcal{R}$.

Assim temos a prova da afirmação.

Agora, cada $\omega_{i}$ é um elemento de $\Gamma$ que não fixa pontos de $\mathbb{R}^{2}$. Logo, dado que $\mathbb{R}^{2} / \Gamma$ é compacto e pelo item número 3 da Proposição 2.2.1, temos que $\omega_{i}$ é de tipo hiperbólico. Portanto existe $x_{i} \in \mathbb{R}^{2}$ tal que $x_{i} \in \operatorname{Min}\left(\omega_{i}\right)$. Seja $\alpha:[0,1] \rightarrow \mathbb{R}^{2}$ uma geodésica minimizante ligando $x_{i}$ e $\omega_{i}\left(x_{i}\right)$, então, pelo item número 2 do Teorema 2.4.1, temos que $\left(\omega_{i},\left.\alpha_{i}\right|_{[0,1]}\right)$ é uma geodésica fechada em $\mathbb{R}^{2} / \Gamma$. As seguintes duas afirmações são propriedades que possui cada elemento da família de geodésicas fechadas $\left\{\left(\omega_{i},\left.\alpha_{i}\right|_{[0,1]}\right)\right\}_{i \in \mathbb{N}}$.

Afirmação 7. Para cada $i \in \mathbb{N}$ a geodésica fechada $\left(\omega_{i},\left.\alpha_{i}\right|_{[0,1]}\right)$ possui um ponto regualr, i.e., existe $t_{0} \in[0,1]$ tal que $\Gamma_{\alpha_{i}\left(t_{0}\right)}$ é trivial.

Primero observemos que a geodésica está definida em todo $\mathbb{R}$, pois $\left(\mathbb{R}^{2}, \mathrm{~g}\right)$ é uma variedade Riemanniana completa. Uma vez que $\left(\omega_{i},\left.\alpha_{i}\right|_{[0,1]}\right)$ é uma geodésica fechada, temos que $\omega_{i} \alpha_{i}(t)=\alpha_{i}(t+1)$ para todo $t \in \mathbb{R}$, i.e., a geodésica $\alpha_{i}(t)$ é invariante por $\omega_{i}$. Portanto, pela Afirmação 4 , temos que imagem $\left(\alpha_{i}(t)\right) \nsubseteq H_{j}^{n}$ para todo $j=1,2,3$ e cada $n \in \mathbb{Z}$. Então existe $t_{1} \in \mathbb{R}$ tal que $\alpha_{i}\left(t_{1}\right)$ é um ponto regular. Agora, dado que $\omega_{i} \alpha_{i}(t)=\alpha_{i}(t+1)$ para todo $t \in \mathbb{R}$, temos que existe $t_{0} \in[0,1]$ e $l \in \mathbb{Z}$ tal que $\omega_{i}^{l} \alpha_{i}\left(t_{0}\right)=\alpha_{i}\left(t_{1}\right)$. Logo $\alpha_{i}\left(t_{0}\right)$ é um ponto regular.

Afirmação 8. Para cada $i \in \mathbb{N}$, temos que a geodésica fechada $\left(\omega_{i},\left.\alpha_{i}\right|_{[0,1]}\right)$ não possui geodésica fechada primitiva distinta dela mesma.

Vamos supor que existe $\bar{\omega}_{i}$ em $\Gamma$ tal que $\left(\bar{\omega}_{i},\left.\alpha_{i}\right|_{\left[0, t_{i}\right]}\right)$ é uma geodésica primitiva associada à geodésica fechada $\left(\omega_{i},\left.\alpha_{i}\right|_{[0,1]}\right)$. Logo, pela Proposição 3.4.5, temos que existem $k$ e $l$ em $\mathbb{N}$ tais que $\omega_{i}^{k}=\bar{\omega}_{i}^{l}$, e portanto $\left(\tilde{\omega}_{i} r\right)^{l}$ é um elemento de $\Gamma_{0}$ Agora, $\bar{\omega}_{i}=\tilde{\omega}_{i} r$, onde $\tilde{\omega}_{i} \in \Gamma_{0}$ e $r \in \mathcal{R}$, onde podemos supor que $\tilde{\omega}_{i}=\omega_{(1)}^{n_{i}} \omega_{(2)}^{l_{i}}$. Seja $x \in \mathbb{R}^{2}$, então $\left(\tilde{\omega}_{i} r\right)^{l}(x)=r^{l}(x)+r^{(l-1)}\left(n_{i}\left(v_{1}+v_{2}\right)+3 l_{i} v_{1}\right)+r^{(l-2)}\left(n_{i}\left(v_{1}+v_{2}\right)+3 l_{i} v_{1}\right)+\cdots+n_{i}\left(v_{1}+v_{2}\right)+3 l_{i} v_{1}$. E portanto $r^{l}=I$, pois $\left(\tilde{\omega}_{i} r\right)^{l}$ é um elemento de $\Gamma_{0}$. 
Logo

$$
\begin{aligned}
r\left(\omega_{i}^{k}(0)\right) & =r\left(k\left(v_{1}+v_{2}\right)+3 k i v_{1}\right) \\
& =r\left(\bar{\omega}_{i}^{l}(0)\right)=r\left(\left(\tilde{\omega}_{i} r\right)^{l}(0)\right) \\
& =r\left[r^{(l-1)}\left(n_{i}\left(v_{1}+v_{2}\right)+3 l_{i} v_{1}\right)+\cdots+n_{i}\left(v_{1}+v_{2}\right)+3 l_{i} v_{1}\right] \\
& =r^{l}\left(n_{i}\left(v_{1}+v_{2}\right)+3 l_{i} v_{1}\right)+\cdots+r\left(n_{i}\left(v_{1}+v_{2}\right)+3 l_{i} v_{1}\right) \\
& =n_{i}\left(v_{1}+v_{2}\right)+3 l_{i} v_{1}+\cdots+r\left(n_{i}\left(v_{1}+v_{2}\right)+3 l_{i} v_{1}\right) \\
& =\left(\tilde{\omega}_{i}^{r}\right)^{l}(0) \\
& =\bar{\omega}_{i}^{l}(0) \\
& =\omega_{i}^{k}(0)
\end{aligned}
$$

Então $r\left(\omega_{i}^{k}(0)\right)=\omega_{i}^{k}(0)$. E assim temos

$$
\begin{aligned}
k r\left(\left(v_{1}+v_{2}\right)+3 i v_{1}\right) & =r\left(k\left(v_{1}+v_{2}\right)+3 k i v_{1}\right) \\
& =r\left(\omega_{i}^{k}(0)\right) \\
& =\omega_{i}^{k}(0) \\
& =k\left(v_{1}+v_{2}\right)+3 i v_{1} \\
& =k\left(\left(v_{1}+v_{2}\right)+3 i v_{1}\right) .
\end{aligned}
$$

Logo $r\left(\left(v_{1}+v_{2}\right)+3 i v_{1}\right)=\left(v_{1}+v_{2}\right)+3 i v_{1}$. Observemos que $\left(v_{1}+v_{2}\right)+3 i v_{1}$ está em $\mathfrak{R}$, e portanto $r=I$. Logo $\bar{\omega}_{i}$ é um elemento de $\Gamma_{0}$. Portanto $\bar{\omega}_{i}=\omega_{i}$, pois $\left(\omega_{i},\left.\alpha_{i}\right|_{[0,1]}\right)$ não possui geodésica primitiva distinta dela mesma em $\mathbb{R}^{2} / \Gamma_{0}$. Logo temos a prova da afirmação.

Afirmaçãa 9. Se $i \neq j$ então as geodésicas fechadas $\left(\omega_{i},\left.\alpha_{i}\right|_{[0,1]}\right)$ e $\left(\omega_{j},\left.\alpha_{j}\right|_{[0,1]}\right)$ são geometricamente distintas em $\mathbb{R}^{2} / \Gamma$.

Vamos supor que $\left(\omega_{i},\left.\alpha_{i}\right|_{[0,1]}\right)$ e $\left(\omega_{j},\left.\alpha_{j}\right|_{[0,1]}\right)$ são geometricamente iguais em $\mathbb{R}^{2} / \Gamma$. Assim, por Proposição 3.4.7, Proposição 3.4 .8 e pela Afirmação 8 , temos que existem $\omega \in \Gamma$ e $b \in \mathbb{R}$ tais que

$$
\alpha_{i}(t)=\omega \alpha_{j}( \pm t+b), \quad \text { para todo } t \in \mathbb{R} .
$$

Então precisamos trabalhar em dois casos.

1. Supomos que

$$
\alpha_{i}(t)=\omega \alpha_{j}(t+b), \quad \text { para todo } t \in \mathbb{R}
$$

Neste caso temos que $\left(\omega_{i},\left.\omega \alpha_{j}\right|_{[b, b+1]}\right)$ é uma geodésica fechada, que é equivalente dizer 
que $\left(\omega^{-1} \omega_{i} \omega,\left.\alpha_{j}\right|_{[0,1]}\right)$ é uma geodésica fechada. Então, usando este último fato e dado que $\left(\omega_{j},\left.\alpha_{j}\right|_{[0,1]}\right)$ é uma geodésica fechada, temos que

$$
\omega^{-1} \omega_{i} \omega \alpha_{j}(t)=\alpha_{j}(t+1)=\omega_{j} \alpha_{j}(t) \text {, para todo } t \in \mathbb{R}
$$

Agora, pela Afirmação 7 , existe $t_{0} \in[0,1]$ tal que $\alpha_{j}\left(t_{0}\right)$ é um ponto regular. Assim, pela equação (3.52), temos que $\omega^{-1} \omega_{i} \omega=\omega_{j}$, mas isto não pode acontecer pela Afirmação 6 .

2. Supomos que

$$
\alpha_{i}(t)=\omega \alpha_{j}(-t+b), \quad \text { para todo } t \in \mathbb{R}
$$

Neste caso vamos ter, então, que $\left(\omega_{i}^{-1},\left.\omega \alpha_{j}\right|_{[b, b+1]}\right)$ é uma geodésica fechada, que é equivalente dizer que $\left(\omega^{-1} \omega_{i}^{-1} \omega,\left.\alpha_{j}\right|_{[0,1]}\right)$ é uma geodésica fechada. Assim, fazendo um raciocínio análogo ao item anterior, vamos obter que $\omega^{-1} \omega_{i}^{-1} \omega=\omega_{j}$. Mas isso é um absurdo pois contradiz a Afirmação 6.

Então, dado que nos dois casos temos uma contradição, temos que $\left(\omega_{i},\left.\alpha_{i}\right|_{[0,1]}\right)$ e $\left(\omega_{j},\left.\alpha_{j}\right|_{[0,1]}\right)$ são geometricamente distintas em $\mathbb{R}^{2} / \Gamma$.

Assim, pela afirmação anterior, podemos concluimos que $\left\{\left(\omega_{i},\left.\alpha_{i}\right|_{[0,1]}\right)\right\}_{i \in \mathbb{N}}$ é uma família de goedésicas fechadas geometricamente distintas em $\mathbb{R}^{2} / \Gamma$. 


\section{REFERÊNCIAS BIBLIOGRÁFICAS}

[AB15] Marcos M. Alexandrino e Renato G. Bettiol. Lie groups and geometric aspects of isometric actions. Springer, Cham, 2015. 3, 4, 21, 26, 30

[AJ11] Marcos M. Alexandrino e Miguel Angel Javaloyes. On closed geodesics in the leaf space of singular Riemannian foliations. Glasg. Math. J., 53(3):555-568, 2011. 3, 45, 46, 55, 58

[BH84] Victor Bangert e Nancy Hingston. Closed geodesics on manifolds with infinite abelian fundamental group. J. Differential Geom., 19(2):277-282, 1984. 71, 98, 101

[BH99] Martin R. Bridson e André Haefliger. Metric spaces of non-positive curvature, volume 319 of Grundlehren der Mathematischen Wissenschaften [Fundamental Principles of Mathematical Sciences]. Springer-Verlag, Berlin, 1999. 38, 46, 60, 77, 98, 120

[Bor92] Joseph Ernest Borzellino. Riemannian Geometry of Orbifolds. Tese de Doutorado, Universidade de California, 1992. 38, 66

[CG72] Jeff Cheeger e Detlef Gromoll. The splitting theorem for manifolds of nonnegative Ricci curvature. J. Differential Geometry, 6:119-128, 1971/72. 63

[CLUV04] Christopher B. Croke, Irena Lasiecka, Gunther Uhlmann e Michael S. Vogelius, editors. Geometric methods in inverse problems and PDE control, volume 137 of The IMA Volumes in Mathematics and its Applications. Springer-Verlag, New York, 2004. 36

[DK00] J. J. Duistermaat e J. A. C. Kolk. Lie groups. Universitext. Springer-Verlag, Berlin, 2000. 3 
[dlH00] Pierre de la Harpe. Topics in geometric group theory. Chicago Lectures in Mathematics. University of Chicago Press, Chicago, IL, 2000. 9, 110

[Dra11] George.C Dragomir. Closed Geodesics on Compact Developable Orbifolds. Tese de Doutorado, McMaster University, 2011. 3, 20, 23, 45

[Dra14] George C. Dragomir. Closed geodesics on orbifolds of nonpositive or nonnegative curvature. Geom. Dedicata, 172:399-411, 2014. 2, 45, 46

[ECH $\left.{ }^{+} 92\right]$ David B. A. Epstein, James W. Cannon, Derek F. Holt, Silvio V. F. Levy, Michael S. Paterson e William P. Thurston. Word processing in groups. Jones and Bartlett Publishers, Boston, MA, 1992. 61

[GH06] K. Guruprasad e A. Haefliger. Closed geodesics on orbifolds. Topology, 45(3):611-641, 2006. $62,63,67$

[GM69] Detlef Gromoll e Wolfgang Meyer. On complete open manifolds of positive curvature. Ann. of Math. (2), 90:75-90, 1969. 61

[Gro73] Karsten Grove. Condition $(C)$ for the energy integral on certain path spaces and applications to the theory of geodesics. 1973. 80

[Kan01] Richard Kane. Reflection groups and invariant theory, volume 5 of CMS Books in Mathematics/Ouvrages de Mathématiques de la SMC. Springer-Verlag, New York, 2001. 32

[Lee11] John M. Lee. Introduction to topological manifolds, volume 202. Second edição, 2011. 3, 13

[LT10] Alexander Lytchak e Gudlaugur Thorbergsson. Curvature explosion in quotients and applications. J. Differential Geom., 85(1):117-139, 2010. 38

[Mun00] James R. Munkres. Topology. Prentice-Hall, Inc., Englewood Cliffs, N.J., second edição, 2000. 88

[Ozo69] Vilnis Ozols. Critical points of the displacement function of an isometry. J. Differential Geometry, 3:411-432, 1969. 80

[P.02] Thurston William P. The Geometry and Topology of Three-Manifolds. Electronic version 1.1, 2002. 3, 22 
[Rat06] John G. Ratcliffe. Foundations of hyperbolic manifolds, volume 149 of Graduate Texts in Mathematics. Springer, New York, second edição, 2006. 3, 9, 10, 14, 16, 17, 31, 33, $36,42,44,105$

[Rot95] Joseph J. Rotman. An introduction to the theory of groups, volume 148 of Graduate Texts in Mathematics. Springer-Verlag, New York, fourth edição, 1995. 98

[Swe99] Eric L. Swenson. A cut point theorem for CAT(0) groups. J. Differential Geom., $53(2): 327-358,1999.62$

[Top68] V. A. Toponogov. The metric structure of Riemannian spaces with non-negative curvature which contain straight lines. páginas 225-239, 1968. 63

[War83] Frank W. Warner. Foundations of differentiable manifolds and Lie groups, volume 94 of Graduate Texts in Mathematics. Springer-Verlag, New York-Berlin, 1983. Corrected reprint of the 1971 edition. 88

[Wol11] Joseph A. Wolf. Spaces of constant curvature. AMS Chelsea Publishing, Providence, RI, sixth edição, 2011. 33, 36 